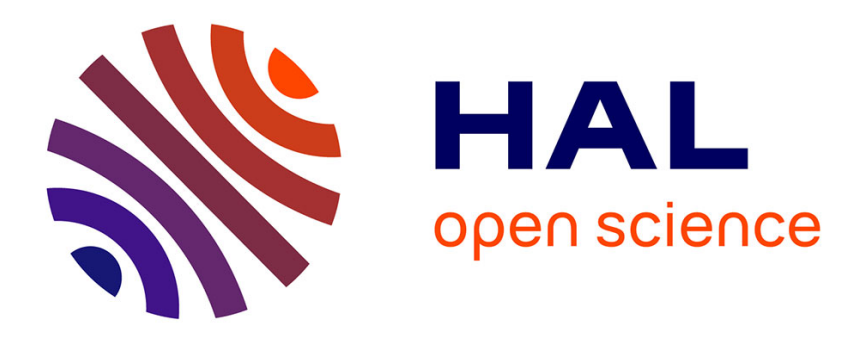

\title{
Simulation modeling in botanical epidemiology and crop loss analysis
}

Serge Savary, Laetitia Willocquet

\section{To cite this version:}

Serge Savary, Laetitia Willocquet. Simulation modeling in botanical epidemiology and crop loss analysis. Plant Health Instructor, 2014, Online, pp.147. 10.1094/PHI-A-2014-0314-01 . hal-02636693

\section{HAL Id: hal-02636693 \\ https: / hal.inrae.fr/hal-02636693}

Submitted on 27 May 2020

HAL is a multi-disciplinary open access archive for the deposit and dissemination of scientific research documents, whether they are published or not. The documents may come from teaching and research institutions in France or abroad, or from public or private research centers.
L'archive ouverte pluridisciplinaire HAL, est destinée au dépôt et à la diffusion de documents scientifiques de niveau recherche, publiés ou non, émanant des établissements d'enseignement et de recherche français ou étrangers, des laboratoires publics ou privés. 


\section{Simulation Modeling in Botanical Epidemiology and Crop Loss Analysis}

\section{Serge Savary and Laetitia Willocquet}

INRA, UMR 1248 AGIR, 24 Chemin de Borderouge - Auzeville, CS 52627, F-31326

Castanet-Tolosan cedex, France

Université de Toulouse, INPT, F-31029 Toulouse, France

\section{Foreword}

Over the years, modeling has become an integral part of plant disease epidemiology (or botanical epidemiology). As in other fields of research, modeling in plant disease epidemiology may serve very different purposes, including:

- synthesizing available data on epidemiological processes;

- predicting epidemiological patterns;

- developing a conceptual framework that captures available data;

- organizing epidemiological knowledge to identify knowledge gaps; or

- designing experiments aimed at testing a theory.

The number of modeling approaches used in plant disease epidemiology has been increasing at a very fast rate. It is not the purpose of this module to review all possible approaches.

This module was developed to highlight, illustrate, and implement the linkages between models and data. Models are necessary to achieve one or several of the objectives listed above using the available data, and data are necessary to both develop and assess models.

Yet, as plant disease epidemiology expands as a field of research, there seems to have been an increasing disconnect between 'field epidemiologists' who collect data and 'epidemiological modelers' who develop models. This gap needs to be filled, because data collection needs to be based on a good understanding of the modeling objective(s) and approach(es), and because model development requires a good understanding of field realities. 
The objective of this module is therefore to bridge the gap between 'observers' and 'modelers'. Among the wide array of possible approaches, we chose one which is particularly visual, involves as little calculus as possible - and thus enables one to concentrate on concepts, and allows the sharing of working examples. We therefore chose the mechanistic simulation approach as a vehicle to address the dynamics of plant disease epidemics and their impact on crop yield.

Serge Savary

Laetitia Willocquet

June 2011, International Rice Research Institute, Philippines

November 2012, Institut National de la Recherche Agronomique, France 


\section{Note for the reader}

This module is designed to empower biologists with a powerful analytical tool. Yet, we have endeavored to develop it with as little calculus and mathematics, and in fact with the hope that readers would discover, enjoy, and explore the field by themselves.

\section{Student}

Even if it is primarily intended to graduate students, this material is intended to undergraduate students too. Undergraduate students will gain some exposure to plant pathology, plant protection, systems analysis, and from a technical viewpoint, to simulation modeling applied to ecological systems. Introductory and transition sections are especially meant to be accessible to a very wide audience. Graduate students will be able to explore this material more in depth.

\section{Instructor}

This module can be used in class as well as for practical work. It provides the basic concepts, methods, and approaches in the field of systems analysis applied to botanical epidemiology. Simulation models are used to that aim, and applied to the dynamics of epidemics and yield losses.

\section{Other reader}

Most of this material is highly visual, and so readily accessible. Although the underpinning concepts can be rather sophisticated, the material assembled here is meant to invite thoughts, and if possible inspire. No prior specific knowledge in calculus, systems analysis, or even plant pathology is required, since these are introduced progressively, when necessary.

\section{Organization and content}

This module is organized as follows:

- A general introduction to simulation models (Chapter 1)

- A presentation of concepts and basic examples (Chapters 2 - 3)

- Simulation modeling in plant disease epidemiology (Chapters 4 - 6)

- Two transition chapters, providing a perspective of simulation modeling in plant disease epidemiology (Interlude) and an introduction to the next sections (introduction to yield loss modeling)

- Simulation modeling of crop growth, yield losses, and their applications to rice and wheat (Chapters 7 - 9)

- A discussion on the concepts associated with model evaluation (Chapter 10)

- Two technical annexes (Instructions to run the simulation models; Simulation models to upload) 


\section{Chapter 1. Simulation Models: Why? Who? When?}

Simulation models have become important tools in Botanical Epidemiology. There are many reasons for this, but we emphasize three of the more important: (1) they enable exploration of hypotheses, and as such, have become invaluable means to guide research; (2) they are unique approaches to integrate (in the literal term of the word) epidemiological knowledge, in the form of experimental results; and (3) they enable connecting epidemiology with other fields of study ranging from agrophysiology to ecology, and from social sciences to natural resource management, for example. This module, and this introductory chapter, is intended to guide the potential user of simulation models. It is not, in any way, meant to be comprehensive on the very diverse simulation tools that already exist, but focuses on mechanistic, dynamic models. Similarly, it is not meant to provide any coverage of the breadth of applications; however, for interested readers, we provide references to use as a possible starting point.

\section{Why use simulation models?}

Simulation models are meant to answer questions which scientists have in a dynamic, quantitative, and often, a pictorial way. Much of the epidemiological research and its applications, in particular, involve a large number of components, actors, and factors. Assembling these in a coherent framework may seem a daunting task, especially for beginners, and can lead to confusion, even for experienced scientists, especially if the objectives of such an exercise are not well defined. This has often resulted in modeling activities becoming an end in themselves, instead of being one of the many tools plant disease epidemiologists may use to analyze and provide answers to crop health problems.

Thus, simulation models have to address specific questions (Zadoks and Rabbinge, 1985), lest becoming self-centered and often unable to bring forward new insights. The insights can be of many kinds. They may be limited to the (very important) objective of better delineating the limits and components of the problem at hand, of identifying key factors that determine the behavior of pathosystems, of deriving disease management options and quantifying their potential efficacy, or of providing a framework for future research, such as, e.g., the quantification of the effects of components of resistance in partial resistance.

Another worthwhile use of simulation modeling is that it still represents today the sole way to numerically integrate the available information often derived from experiments on processes 
underpinning plant disease epidemics. This type of application has the important value of enabling a numerical visualization of knowledge gaps.

Simulation models enable mobilizing available (primarily quantitative) knowledge of a system, and exploration of a system's behavior. This property of simulation modeling is derived from the link between integration levels in biological systems (Rabbinge and De Wit, 1989), that is, the fact that simulation models are based on the principle that the behavior of a system at one level of integration (say, a field) is a reflection of processes operating at the next-lower level of biological integration (e.g., plants, diseased or healthy), that form the population that is present in the field. In other words, simulation enables upscaling, that is, the integration of processes occurring at a given level of hierarchy within a system (e.g., 'a site on a leaf is infected') to a higher scale of hierarchy of that system (e.g., 'an epidemic takes place'). As a result, simulation modeling is unique as a scientific approach, because it enables one to explore possible futures. Of course, simulation modeling is a key approach currently used to study the effects of climate change on earth's systems, including plant disease epidemics. There is however a large number of other applications of the extrapolation power of simulation modeling. This was already recognized by pioneers in the field, who actually conducted simulation-based experiments in botanical epidemiology a long time ago (Teng, 1985).

Critical to the approach, however, is to specify the purpose of modeling prior to engaging into the modeling work. This implies that one has to choose among the many applications of modeling. Defining the purpose of modeling, in many ways, entails the underpinning question of model evaluation - will the anticipated model be evaluated? In which way? Do data exist that are appropriate for model evaluation available? Model evaluation is a scientific field of its own (Teng, 1981), but is first a philosophical scientific issue: models, including simulation models, only consist of carefully chosen components of a system; and a system is a simplification of (modeled) reality.

Thus, (simulation) models can only be proven wrong, to some degree. The notion of 'validation' applied to models (as well as to theories in general, Popper, 1963) might imply that a model is 'true', while the only truth is the reality, of which models are only simplifications. In many ways, therefore, scientists might be more interested in developing simple, parameter-sparse models, that can easily be evaluated, whether in terms of their inherent consistency (the model operates as the investigator intends it to), or in terms of their outcomes (the model's outputs reasonably match the available observation). Teng (1981) provides an important discussion on the philosophical background of model evaluation.

Another reason for investigators to favor parameter-sparse models emerges when models are intended for applied purposes: in such a case, one will often choose a model that requires little 
information to operate, so as to be accessible to the largest number of users, in the broadest range of contexts.

It follows from the above remarks that, as in experimental research, the simplest model enabling the investigator to (1) better understand the behavior of a system, and (2) clearly answers specified questions is often to be preferred to complicated structures. The latter are difficult to evaluate, complex in their inherent behavior, and often very difficult to use for practical plant protection purposes. Therefore, a simple, clear, and easily shared model reflects that the question addressed has been clearly expressed.

\section{Who are the users of simulation models?}

Development of simulation models does not require mathematical and programming expertise. But it does require (1) a good understanding of the system under consideration, (2) some basic knowledge of calculus, and, again, (3) a good articulation of the scientific question at hand.

For botanical epidemiologists, critical steps thus are to (1) clearly specify the objectives of simulation modeling, (2) have a good outline of the system to address (this will be addressed in the next chapter) with numerical information of the next-lower level of integration (e.g., the monocyclic processes of epidemics), and (3) match the above two points with independent available data that pertain to the level of integration to be modeled (e.g., an epidemic), in order to enable the evaluation of the model that has been developed.

\section{When to use simulation models?}

The use of simulation becomes apparent as soon as a number of factors are considered to influence the behavior of a system. Many approaches, especially statistical ones, are available to analyze interactions in (biological) systems. Simulation modeling constitutes a unique approach in that it enables the simultaneous handling of a range of such factors and 'see' their influence on the behavior of a system, such as the course of an epidemic. In addition to the "Why" reasons listed above, one key outcome of simulation modeling is a better understanding of which components of a (plant-pathogen) system are truly important in its behavior, and which are presumably less so.

In plant pathology, especially in botanical epidemiology, as well as in the analysis of the translation of epidemics into crop losses, one is always dealing with dynamic processes. Simulation modeling is a powerful approach to address such processes. Over the course of time, some components 
of a system will have an increasing, or decreasing, effect on the behavior of the considered system (e.g., the dynamics of an epidemic, or the build-up of yield - and thus of yield losses - over time). Anticipating such switches, especially when combined with factors that are considered important in the properties of the considered system (e.g., environmental or man-made factors) is extraordinarily difficult. Simulation modeling provides a unique way to visualize, understand, and quantify such dynamics.

Lastly, simulation models can also represent good educational tools: they can provide an intuitive hands-on analysis of (plant-disease) systems.

\section{Summary}

- Simulation models have a number of applications. In particular, simulation models:

○ allow exploration of the behavior of plant-pathogen systems;

$\circ$ in doing so, they enable mobilizing experimental data;

○ enable exploring the sensitivity of plant-pathogen systems to some of their (specified) components;

○ allow exploration of "futures", i.e., analyze how the considered system might behave under yet-undocumented conditions.

- Development of simulation models does not require mathematical and programming expertise. But it does require (1) a good understanding of the considered system, (2) some basic knowledge of calculus, and (3) a good articulation of the scientific question at hand.

- Simulation modeling is a powerful approach to address dynamic processes. Over time, some components of a system may have stronger, or weaker, effects on the behavior of a system (e.g., the dynamics of an epidemic, or the build-up of yield - and thus of yield losses). Such questions can powerfully be addressed through simulation modeling.

- Simulation models are good educational tools: they can provide an intuitive hands-on on analyzing (plant-pathogen) systems.

\section{References}

Popper, K. R. 1963. Conjectures and Refutations: The Growth of Scientific Knowledge. Routledge, London.

Rabbinge, R., and De Wit, C.T. 1989. Systems, models and simulation. Pages 3-15 in: Simulation and Systems Management in Crop Protection. Rabbinge, R., Ward, S.A. and Van Laar, H.H., Eds. Pudoc, Wageningen, $420 \mathrm{p}$. 
Teng, P.S. 1981. Validation of computer models of plant disease epidemics: a review of philosophy and methodology. Zeitschrift für Pflanzenkrankheiten und Pflanzenschutz 88:49-63.

Teng, P.S. 1985. A comparison of simulation approaches to epidemic modeling. Annual Review of Phytopathology 23:351-379.

Zadoks, J.C., and Rabbinge, R. 1985. Modelling to a purpose. Pages 231-244 in: Advances in Plant Pathology, Vol. 3. Mathematical Modelling of Crop Diseases. C.A. Gilligan, ed. Academic Press, London. 


\section{Chapter 2. Systems, Models, and Simulation}

\section{A few definitions}

A system is a simplified representation of reality. "System" is a common word, often used with loose meaning. Whereas in the real world, a "system" may seem at times an endless series of connected elements, we refer here to a system as (1) a series of selected, chosen elements (this is a first simplification, and thus an implicit assumption), with (2) specified boundaries (a second simplification and implicit assumption), and (3) pre-determined time characteristics (with a third simplification and implicit assumption).

These simplifications over space and time are important: they require pondering, and thus, expertise on the reality at hand. A 'simple' system could for instance be a nearby coffee shop. This coffee shop has customers who place orders and staff who process them. There may be at times very few customers, whereas at others, the place is very busy (say, because the coffee shop is just nearby the University, and has free wi-fi, which the students use while enjoying a coffee and chat with their friends). So, for the customers, and the staff too, time is not neutral. It is then useful to look at our coffee-shop-system over a series of sections of time (time steps) that make a day. Perhaps an appropriate time step of one hour is adequate: it is more than enough to encapsulate long hours when little really happens, but is just enough to capture events at peak time. So much, though, may happen in one hour over a cup of coffee, when the place is busy, people meet, many orders are placed, many messages received. Perhaps, a time step of 30 minutes, or even 15 minutes might then be better. So, although many near-empty 15 -minute segments might be a waste of computing time, and lead to outputs that may be boring for some parts of the day, these might ensure that important events are not lost at peak time. Yet - so many things may still happen over a period of 15 minutes. Might a time step of 5 minutes be safer? This is obviously not an easy question.

At any rate, a decision must be made, and it is up to the modeler to make it. Each system, such as the coffee-shop-system, has a time constant, which we can simply define for the time being as the delay over which the system may strongly change, or, in systems analysis phrasing: over which the state of the system may change. One way to empirically choose a time constant is based on experience and knowledge of the system at hand. Note that in the coffee-shop-system, not all the elements are enclosed within the coffee shop itself, which are important for the coffee-shop-system: for instance, it has free wi-fi. We therefore can call it a semi-open system. Biological systems, phytopathological systems in 
particular, are semi-open: they receive and transmit information, components, biomass, or energy from and to their environment.

A model is a computer program that describes the mechanics of the considered system. The encoding of a model can be made in many ways. Here, we use the STELLA ${ }^{\circledR}$ program, which enables us to focus only on components of a system, the system's structure, relationships among components, and the modeled system's behavior, rather than on the code of the program itself. Here, we refer to dynamic simulation models. At each time step, the status of the system changes: in the coffee-shopsystem, customers come and go, orders are placed, coffee is drunk, receipts are paid, messages are received, news is shared, sometimes coffee is spilled on computers. At each time step, the model updates the status of the system, and is set ready to account for the events of the following time step on the basis of the new status it just has acquired.

A simulation, simply, is the execution of a model. This requires the further definition of the initial conditions of the system under consideration, and specified values of parameters. Again, this implies expertise on the system at hand. In the coffee-shop-system, one has to decide a few things. When in the day does the modeling start? How many customers and staff are already there at that time? How much money does the cashier have then? What are the prices of the different kinds of coffee? What are the rates of inflow and outflow of customers (and what determines it)? What is the rate of inflow of messages? The modeler, simply, has to set the scene, and decide a few rules. These may be made simple in the beginning.

\section{A preliminary warning remark}

The beginning of the previous paragraph started with three elements of simplification regarding the components of a system, the boundary within which a system operates, and the time characteristics of the system under consideration. As in other branches of science, these simplifications are made in order to make the problem tractable. Before we proceed, it is very important to stress that, while science progresses through assumptions (and thus simplifications) that are tested and refuted, such simplifications in modeling do correspond to assumptions. One real danger of modeling without revisiting such assumptions is to make the process of model development, verification, and evaluation an exercise which becomes disconnected from the reality at hand. These simplifications-assumptions are derived from the modeler expertise of a reality, and of hypotheses about components that govern the 
system's behavior. Not revisiting (and testing) these hypotheses may lead to reductionism, not to the originally intended 'systems approach'.

\section{Analytical and numerical integration: example of the exponential growth}

Some basics of calculus are needed for modeling. But this is very little indeed. A classical example is that of exponential growth. If one considers a system in which an organism (say, a bacterium) is provided with unlimited nutrient and conditions that are suitable for its maintenance, growth, and multiplication, then exponential growth is expected to occur. Such a system is of course a very strong simplification of reality, and we shall come back to addressing such simplification in the following chapter.

Nevertheless, let us assume that this system is worthwhile considering for now, and let us denote by $x$ the number of bacteria, and by $t$, the elapsing time. What follows are two approaches to modeling the system. We shall start with an analytical integration. We then will address the same question with what is called a numerical integration. This section will end with a brief discussion on the differences between these two approaches.

The analytical integration of the problem could be written as follows. Let us consider a very simple process, whereby a given quantity, $x$, increases over time, $t$, with a given rate, $r$. Let us further assume that $x$ may vary between an initial value, $x_{0}$, and a final value $x_{f}$ Conversely, time may vary between a starting time, $t_{0}$, and a final time $t_{f}$, so that one can write:

$$
x \in\left\lfloor x_{0}, x_{f}\right\rfloor
$$

and

$$
t \in\left\lfloor t_{0}, t_{f}\right\rfloor .
$$

Therefore, one can write that the variation in $x$ relative to any variation (more precisely, to any infinitely small variation) of time, $d t$, is proportional to $r$ and to the value of the currently existing quantity $x$ :

$$
d x / d t=r * x
$$

One can note that the quantity $d x / d t$ is a ratio between the considered quantity and time. If, for example, $x$ were a distance, $d x / d t$ would represent the physical speed of an object. In general, these equations refer to some speed of a sort; we shall come back to that point later-on. 
This differential equation can be easily handled by 'moving' the $d t$ term to the right hand-side of the equation, and the $x$ term to the left hand-side of the equation, so:

$d x / x=r * d t$

Now that we have all the $x$ s on the left hand-side, and the time term, $d t$, on the right hand-side of the equation, we can use Riemann integrals in order to solve this differential equation, and write:

$$
\int_{t_{0}}^{\tau} d x / x=\int_{t_{0}}^{\tau} r * d t
$$

where $t_{0}$ and $\tau$ denote the initial and final times over which the process is integrated, respectively.

We may assume at this stage that $r$, the rate of increase of the quantity under consideration, is constant over the time interval we have chosen $\left[t_{0}, \tau\right]$. With this assumption, $r$ can be extracted from the integral sign of the right hand-side of the equation:

$$
\int_{t_{0}}^{\tau} d x / x=r \int_{t_{0}}^{\tau} d t
$$

The integration of both sides of the equation can then be done, as:

$$
[\ln (x)]_{t_{0}}^{\tau}=r *[t]_{t_{0}}^{\tau}
$$

where the $t_{0}$ and $\tau$ signs on both sides of the brackets indicate, as before, the initial and final times when the integration is made. This translates into:

$$
\ln \left(x_{\tau}\right)-\ln \left(x_{t_{0}}\right)=r *\left(\tau-t_{0}\right)
$$

which amounts to:

$$
\ln \left(x_{\tau} / x_{t_{0}}\right)=r *\left(\tau-t_{0}\right)
$$

If we simplify the way to write variables as:

$$
x_{\tau}=x_{\mathrm{t}} ; x_{\mathrm{t} 0}=x_{0} ;
$$

as well as considering that the running time, $\tau$, can be written as $t: \tau=t$; and if we assume that the initial time is null: $t_{0}=0$, we can make further simplifications to the equation:

$$
\ln \left(x_{\mathrm{t}} / x_{0}\right)=r * t
$$

The reverse function of the natural logarithm is an exponential, so that we can write:

$$
x_{\mathrm{r}} / x_{0}=\exp (r * t)
$$

And so, we can write:

$$
x_{\mathrm{t}}=x_{0} * \exp (r * t)
$$


This is the typical exponential growth function, which states that the population of bacteria, $x$, increases exponentially, with an initial value of $x_{0}\left(\right.$ when $\left.t=0, \mathrm{e}^{r_{*}^{* t}}=\mathrm{e}^{0}=1\right)$, and this growth is infinite.

The numerical integration of the same problem can now be addressed as follows. Let us say that:

- the amount of bacteria is to be denoted by A, the number of bacteria in the system at a given point of time;

- the rate of increase of the bacterial population is denoted RA; and

- the relative rate of increase of the bacterial population, that is, the rate of increase of the bacterial population relative to the amount of bacteria present in the system is denoted RRA.

One notes that, in comparison with the analytical integration, we now have:

$$
\begin{aligned}
& \mathrm{A}=x ; \\
& \mathrm{RA}=d x / d t, \text { and } \\
& \mathrm{RRA}=(d x / d t) / x .
\end{aligned}
$$

The numerical integration of this problem only involves two lines of code:

$$
\begin{aligned}
& \mathrm{A}(\mathrm{t}+\Delta \mathrm{t})=\mathrm{A}(\mathrm{t})+\mathrm{RA} * \Delta \mathrm{t} \\
& \mathrm{RA}=\mathrm{RRA} * \mathrm{~A}
\end{aligned}
$$

The first equation states that at each time step, $\Delta \mathrm{t}$, the amount $\mathrm{A}$ of bacteria at time $\mathrm{t}, \mathrm{A}(\mathrm{t})$, is incremented by the quantity RA * ${ }^{*}$, and the second, that RA is in turn the product: RRA * A.

These equations can also be summarized by a diagram:

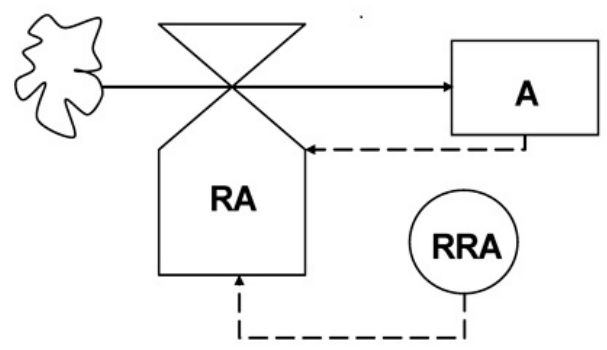

Figure 2.1. A flowchart for exponential growth. The amount of bacteria is denoted $A$, the rate of bacterial increase is denoted RA at each time step $\Delta \mathrm{t}$, with a relative (or intrinsic) rate RRA (See also Table 2.2).

One may want to consider if, and to what extent, the principle of the two methods, analytical and numerical integration, differ. Let us come back to the differential equation with which we started: 
$d x / d t=r * x$

If, in this equation, one replaces the infinitely small differences, denoted $d \bullet$ in bacterium numbers $(d x)$ or in elapsed time $(d t)$, by small variation in bacterium number, $\Delta \mathrm{x}$, in response to small variation in time, that is, by time step $\Delta \mathrm{t}$, one would derive:

$$
\Delta x / \Delta t=r * x
$$

Let us make $\Delta \mathrm{t}$ approaching infinitely small values, that is, let us consider the limit of the ratio $\Delta \mathrm{x} / \Delta \mathrm{t}$ with $\Delta \mathrm{t}$ becoming infinitely small. One may write:

$$
\lim _{\Delta_{\mathrm{t} \rightarrow 0}}(\Delta \mathrm{x} / \Delta \mathrm{t})=d x / d t
$$

where $d x / d t$ is the definition of the derivative of $x$ over $t$. In other words, writing:

$$
\Delta x / \Delta t=r * x
$$

is formally incorrect, but one may say that the ratio $\Delta \mathrm{x} / \Delta \mathrm{t}$ is an approximation of $r * x$, if $\Delta \mathrm{t}$ is small enough. One should thus write:

$$
\Delta x / \Delta t \approx r * x
$$

The two approaches therefore are not identical. The formal analytical integration yields the correct result, whereas the numerical integration only provides a numerical estimate. Science of course prefers exact results. Some systems, however, are sufficiently complicated to prevent the derivation of an analytical solution. Should such systems be disregarded for this reason? Numerical integration provides a means to produce approximate solutions. For instance, in the above example, the analytical solution was derived while assuming $r$ constant. This of course very seldom happens, even in highly simplified systems. Numerical integration provides a simple way to address variation over time of parameters such as, in this example, $r$. Furthermore, sources of variation other than time can be addressed as well. This will be addressed in the next chapter.

Numerical integration also provides other, quite important, advantages including: (1) means to easily explore the behavior of a system, and (2) means to easily develop, convey, and share model structures and their implications, as we shall try to show.

As pointed out in the first section of this chapter, one must bear in mind elements pertaining to (1) the implicit assumptions-simplifications that form the basis of a model structure, (2) the need for expertise when time steps, systems limits, and systems components are chosen, and (3) the necessity to suitably assess simulation model outputs. Such precautions are needed irrespective of the modeling approach chosen. 


\section{Forrester's symbols and syntax}

Table 2.1 provides a summary of the symbols Jay Forrester (1961) created, which were used previously in Fig. 2.1 and which will be used throughout the module.

Table 2.1. List of symbols for simulation modeling. After Forrester (1961).

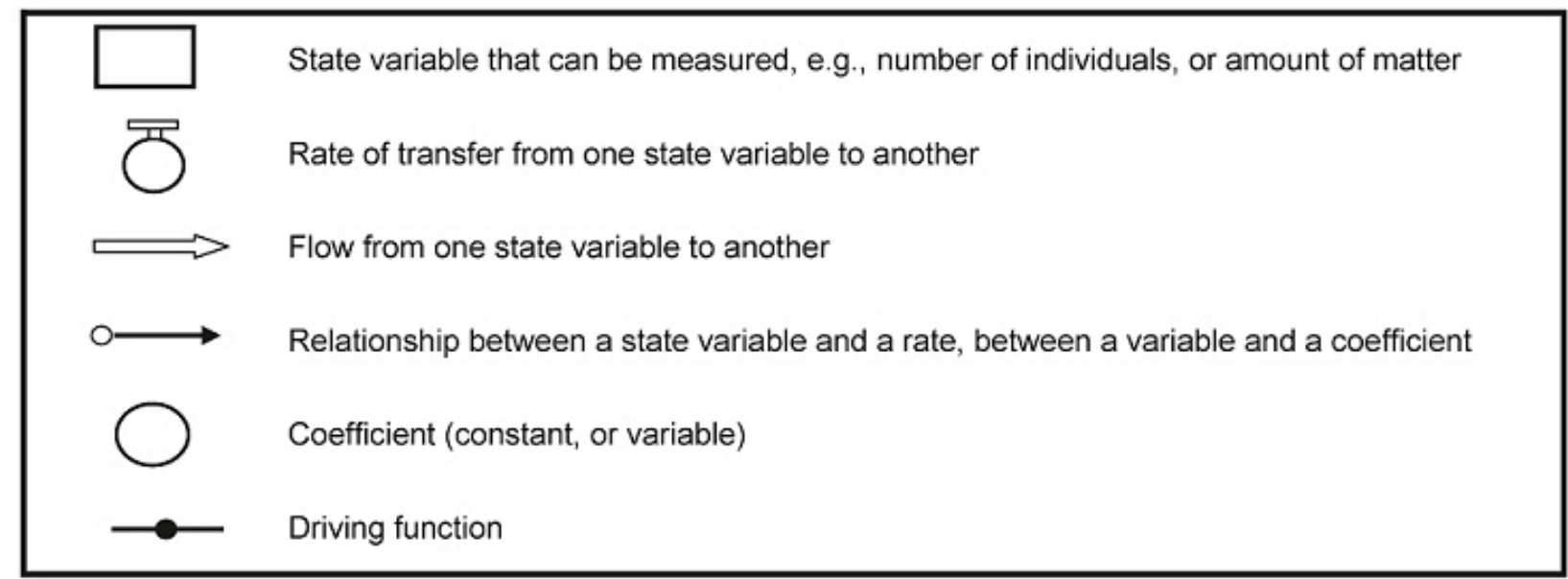

The first symbol is a rectangle, representing a state variable. State variables characterize a system's status, and are continuously varying in the system. In the above example, the state variable is A, or the number of bacteria. Surprisingly enough, the choice of state variables is critical, and also reflects the interests of the modeler. In the virtual coffee-shop system of the former section, several choices could be made. For instance, a specialist in population dynamics (or professors concerned by attendance in class) would probably choose state variables which express numbers of customers (i.e., which have 'numbers' as dimension, as discussed below); an economist would perhaps choose state variables expressing money exchanged; an information theory specialist might choose state variables representing information in its various forms; or a supply-chain expert might consider stocks of coffee in their various stages of consumption. Such choices have implications on the very use of the model, of course, but also may lead to pondering the limits of the system to consider (what is the limit of information? where does the coffee actually come from?), the flows and connections the system involves, as well as its time-constant. While such choices are in the hands of the modeler, a rule of thumb exists: a 'good' mechanistic model is one which has state variables that have correctly been chosen, because the state of the system is described by several state variables (accounting for a series of relevant transitions in one key component of the system, say, the numbers of incoming, waiting, sitting, paying, and leaving customers), and comparatively few parameters. 
This last point brings us to what we feel is a critical remark, although many might perceive it as obvious: modeling must have a purpose. One is often tempted to model 'everything', that is, mix up levels of integrations (e.g., the life cycle of an individual lesion, the dynamics of disease on a plant, the dynamics of disease in a canopy or a landscape, as well as the crop losses caused by disease). This is a very dangerous path to take: systems analysis tells us that the behavior of a system at one level of integration depends on processes occurring at the immediately lower level of integration. Limits must be chosen, and objectives set. The choices of the state variables, of the limits of the system, for instance, are important steps to not drifting towards unmanageable complication. Setting such limits also allows focusing on the applications a model may have.

The second symbol is a valve which controls a flow incoming or leaving a state variable; this symbol is always connected to the very flow the valve controls, the third symbol of Table 2.1. There can be only one valve, that is, one control, per flow. Flows are represented in solid arrows (Fig. 2.1) or double lines (Table 2.1). They represent the increase, or decrease, of contents of the state variable the flow reaches or leaves. In Fig. 2.1, rate RA controls the inflow of bacteria into the state variable A, the total number of bacteria in the system.

Systems nearly always involve flows other than those pertaining only to the increase or decrease in contents of state variables. These flows of information are shown in dashed lines (Fig. 2.1) or in simple thin lines (Table 2.1). A flow of information always originates from a coefficient, a (possibly variable) parameter, a driving function, or from a state variable, as in Fig. 2.1.

Coefficients or parameters are shown as circles, as in Fig. 2.1, where RRA represents the relative rate of bacteria increase.

The last symbol introduced by Forrester (Table 2.1) is that for a driving function: a segment and a dot at its middle. This brings us back to the beginning of this chapter, when dealing with semi-open systems. Driving functions are meant to represent factors that are not included within the set boundaries of the considered system, but nevertheless, influence it from the outside. Examples for driving functions are many: the Earth system does not include the Sun, yet everything that happens on Earth depends on the radiations intercepted by Earth from the Sun, which therefore may be represented by a driving function; or, the purchasing behavior of customers in the coffee-shop-system may depend on the price of the coffee - or on whether examination dates are approaching. Similarly, in Botanical Epidemiology, the behavior of a pathosystem may strongly depend on temperature or rainfall. Driving functions 
represent variables that are outside the limits of the considered system, and yet may strongly influence it. They also are likely to vary strongly, and the choice of a suitable time step has to take into account these variations. Some programs, such as the STELLA® program, represent driving variables with the same symbol as (variable) parameters, i.e., circles. However, it is important to bear in mind the clear difference between a parameter (within a system) and a driving function (outside its boundaries).

\section{Dimensions}

Dimensions can be represented between brackets. For instance, $[\mathrm{L}],[\mathrm{T}]$, and $[\mathrm{K}]$ stand for length, time, and temperature dimensions, respectively. The speed of an object, for example, would have dimension: $\left[\mathrm{L} . \mathrm{T}^{-1}\right]$, that is, distance per unit of time:

speed $=$ distance $/$ time

with dimensions: $\left[\mathrm{L} \cdot \mathrm{T}^{-1}\right] \equiv[\mathrm{L}] /[\mathrm{T}] \equiv[\mathrm{L}] \cdot\left[\mathrm{T}^{-1}\right]$.

Note that the symbol between $\mathrm{L}$ and $\mathrm{T}^{-1}$ does not represent a multiplication sign in the algebraic sense.

An equation such as: $\mathrm{RA}=\mathrm{RRA} * \mathrm{~A}$ in the above example entails dimensions.

- A, the size of the bacterial population has for dimension: [bacteria], or: [N];

- RA, the rate of growth of the bacterial population has for dimension: [bacteria.time ${ }^{-1}$ ], or in a simplified manner: $\left[\mathrm{N} \cdot \mathrm{T}^{-1}\right]$; and

- RRA, the rate of growth of the bacterial population relative to the bacterial population size has for dimension: [bacteria.bacteria ${ }^{-1} \cdot$ time $\left.^{-1}\right]$, or: $\left[\mathrm{N} \cdot \mathrm{N}^{-1} \cdot \mathrm{T}^{-1}\right]$

The dimensionality of the equation:

$\mathrm{RA}=\mathrm{RRA} *$ A therefore is: $\left[\mathrm{N} \cdot \mathrm{T}^{-1}\right] \equiv\left[\mathrm{N} \cdot \mathrm{N}^{-1} \cdot \mathrm{T}^{-1}\right]^{*}[\mathrm{~N}]$.

Since the number dimensions, $[\mathrm{N}]$ and $\left[\mathrm{N}^{-1}\right]$, cancel one another in the right hand side of the dimensionality equation, one thus can see that both sides of the equation for RA have the same dimensions.

Checking the dimensionalities of an equation is one good way to check if the equation itself is correct. Do note, however, that the reverse is incorrect: identical dimensionalities of both sides of an equation are no proof of its correctness (and of course not of its scientific validity). Nevertheless, it is a very convenient way to check for gross mistakes.

Unlike analytical integration, numerical integration therefore deals with dimensions. In particular, the dimension of the state variables that are involved in a model is one key additional 
decision a modeler must make. In that sense, numerical integration brings us close to the realm of physical sciences, although of course mathematical correctness is required. Choosing, checking, and pondering the dimensions of each of the elements of a model does not cause additional trouble. On the contrary, it provides a critical instrument to control whether the modeling structure is consistent. This is particularly useful when a model involves a number of state variables, rates, parameters, and driving functions. Note that dimensions are related to units. However, a given dimension may correspond to different units, and the latter should of course be consistent across the structure of a model as well.

Table 2.2 provides a list of dimensions for state variables, rates, and coefficients. Note, as indicated above, that all the rate variables are actually speeds of some sort, and thus have dimensions: [ -. $\left.\mathrm{T}^{-1}\right]$.

Table 2.2. Dimensions for a set of examples of variables

\begin{tabular}{|c|c|c|c|}
\hline $\begin{array}{l}\text { Variable } \\
\text { type }\end{array}$ & Variables & Dimensions & $\begin{array}{l}\text { Units } \\
\text { (examples) }\end{array}$ \\
\hline \multirow{7}{*}{$\begin{array}{l}\text { State } \\
\text { variables }\end{array}$} & Length & [L] & $\mathrm{m}$ \\
\hline & Mass & {$[\mathrm{M}]$} & $\mathrm{kg}$ \\
\hline & Number (population size) & {$[\mathrm{N}]$} & number \\
\hline & Leaf area & {$\left[\mathrm{L}^{2}\right]$} & $\mathrm{m}^{2}$ \\
\hline & Leaf area index & {$\left[\mathrm{L}^{2} \cdot \mathrm{L}^{-2}\right] \equiv[1]$} & $\mathrm{m}^{2} \cdot \mathrm{m}^{-2}$ \\
\hline & Root length & [L] & $\mathrm{m}$ \\
\hline & Population deny & {$\left[\mathrm{N} . \mathrm{L}^{-2}\right]$} & number $\cdot \mathrm{m}^{-2}$ \\
\hline \multirow[t]{3}{*}{ Rates } & Speed & {$\left[{\left.\mathrm{L} . \mathrm{T}^{-1}\right]}^{-1}\right.$} & $\mathrm{m} \cdot \mathrm{s}^{-1}$ \\
\hline & Growth & {$\left[\mathrm{M} . \mathrm{T}^{-1}\right]$} & $\mathrm{kg} \cdot \mathrm{d}^{-1}$ \\
\hline & Population increase & {$\left[\mathrm{N} . \mathrm{T}^{-1}\right]$} & number $\cdot \mathrm{d}^{-1}$ \\
\hline \multirow[t]{3}{*}{ Coefficients } & Acceleration & {$\left[\mathrm{L} . \mathrm{T}^{-1} \cdot \mathrm{T}^{-1}\right] \equiv\left[\mathrm{L} . \mathrm{T}^{-2}\right]$} & $\mathrm{m} \cdot \mathrm{s}^{-2}$ \\
\hline & $\begin{array}{l}\text { (Bio)mass relative growth } \\
\text { rate }\end{array}$ & {$\left[\mathrm{M} \cdot \mathrm{M}^{-1} \cdot \mathrm{T}^{-1}\right] \equiv\left[\mathrm{T}^{-1}\right]$} & $\mathrm{d}^{-1}$ \\
\hline & $\begin{array}{l}\text { Relative population growth } \\
\text { rate }\end{array}$ & {$\left[\mathrm{N} \cdot \mathrm{N}^{-1} \cdot \mathrm{T}^{-1}\right] \equiv\left[\mathrm{T}^{-1}\right]$} & $\mathrm{d}^{-1}$ \\
\hline
\end{tabular}




\section{Time constant and integration step}

Let us return to the notion of time constant. As the model runs, a program is executed. Its execution is based on a chosen time step, $\Delta \mathrm{t}$. At each time step during the running time of the program, each state variable at $t+\Delta t$ equals the value of the state variable at time $t$, plus the rate at time $t$ multiplied by $\Delta \mathrm{t}$. This procedure of numerical integration yields the new values of the state variables.

The time step of the model, $\Delta \mathrm{t}$, has to be chosen small enough so that the rates do not change notably within $\Delta$ t. To avoid instability, the time step has to be much smaller than the time constant of the considered system. The time constant of a very simple system such as the bacterial population model considered in this chapter is $1 /$ RRA (note that: $1 /$ RRA $\equiv[T]$ ).

Depending on authors, the time step used should be $1 / 3^{\text {rd }}$ to $1 / 5^{\text {th }}$ of the system's time constant. Most systems, however, involve several processes, and therefore, several rates. One may consider that the time constant of such a system is equal to the reverse of the fastest relative rate of change of one of its state variables. The smaller the time constant of a system, the smaller the time step will have to be.

\section{Summary}

This chapter introduces the concepts of system, model, and simulation. It also

- introduces the notion of numerical integration, and compares it with analytical integration;

- thus, the notion of time step, its choice, and the concept of time constant are introduced;

- by means of a simple exponential process, the syntax of Forrester to represent systems is introduced;

- and the notion of dimensionality of variables and parameters in a model is explained.

\section{References}

Forrester, J.W. 1961. Industrial Dynamics. M.I.T. Press, Cambridge (Mass.).

Penning de Vries, F.W.T., and Van Laar, H.H., eds. 1982. Simulation of Plant Growth and Crop Production. Pudoc, Wageningen.

Rabbinge, R., Ward, S.A., and Van Laar, H.H.,eds. 1989. Simulation and Systems Management in Crop Protection. Pudoc, Wageningen.

Thornley, J.H.M., and France, J. 2007. Mathematical Models in Agriculture. Quantitative Methods for the Plant, Animal, and Ecological Sciences. $2^{\text {nd }}$ Ed. CABi, Wallingford. de Wit, C.T., and Goudriaan, J.G. 1978. Simulation of Ecological Processes. Pudoc, Wageningen. 


\section{Suggested reading}

Case, J.T. 2000. An Illustrated Guide to Theoretical Ecology, Oxford University Press, New York.

May, R., and McLean, A. 2007, Theoretical Ecology, Third Edition. Oxford University Press, New York.

Renshaw, E. 1991. Modelling Biological Populations in Space and Time. Cambridge University Press, Cambridge.

\section{Exercises and questions}

1. A reasonable time step to simulate the dynamics of the number of books in a library is
a. one second
b. one day
c. one month
d. one year

2. What are reasonable time steps in the coffee shop if one chooses:
a. the number of customers as state variable;
b. the number of coffee cups served as a state variable;
c. the number of incoming and outgoing e-mails as a state variable;
d. the number of employees present at any time in the coffee shop;
e. the amount of money in the cashiers desk at any time.

3. In the modeling of growth of a bacterial population, the rate of growth of the bacterial population, the relative rate of growth of the population, and the number of bacteria are, respectively:
a. a state variable, a rate, and a relative rate;
b. a rate, a relative rate, and a state variable;
c. a relative rate, a rate, and a state variable.

4. A state variable is

a. A rate of change of variable

b. A constant parameter 
c. A variable which varies at each time step, depending on inflows and outflows

d. A driving function

5. Numerical integration
a. can be done when parameters vary over time
b. is identical to analytical integration
c. requires mathematical integration
d. does not depend on the integration time step

6. The dimension of speed is
a. [L]
b. $\left[\mathrm{L}^{2}\right]$
c. [L.T]
d. $\left[\mathrm{L} \cdot \mathrm{T}^{-1}\right]$

7. The dimension of the density of bacteria in a suspension is
a. $\left[\mathrm{T}^{-1}\right]$
b. $\left[\mathrm{N} \cdot \mathrm{L}^{-3}\right]$
c. $\left[N . L^{-2}\right]$
d. $\left[\mathrm{L} . \mathrm{T}^{-1}\right]$

\section{Answers to exercises and questions}

1. b: one day

2. Reasonable time steps are in the range of:
a. for the number of customers: 1 hour;
b. for the number of coffee cups served: 5 minutes;
c. for the number of incoming and outgoing e-mails: 1 minute;
d. for the number of employees present at any time in the coffee shop: 1 hour;
e. for the amount of money in the cashiers desk at any time: 1 hour or 1 day. 
3. b: a rate, a relative rate, and a state variable.

4. c: A variable which varies at each time step, depending on inflows and outflows

5. a: can be done when parameters vary over time

6. d: $\left[\right.$ L.T $\left.T^{-1}\right]$

7. b: $\left[N . L^{-3}\right]$ 


\section{Chapter 3. Preliminary Examples of Simulation Models}

This chapter introduces some of the main elements required to develop models and explore their behavior. The development of a model amounts to the articulation of hypotheses pertaining to a system under study. One (but not the only one) of the main interests in developing models is to 'see' how the system would behave, assuming that the representation we make of that system is correct.

Here, we use the STELLA® program, because it (almost) directly uses Forrester's symbols, and because we do not have to bother about the syntax of the program. We do have, however, to be consistent with respect to the syntax of Forrester's symbols, which one can consider as pictograms, each of them corresponding to individual computing steps (see previous section). As will be shown in the following text, the choice of this programming platform sets us free from computing details, and instead allows focusing at the system at hand, its time and space characteristics, the way we would like to represent it, and then on the set of equations we believe govern its functioning.

Essentially, STELLA® operates in five stages:

1- draw the elements and relationships that represent the structure of the model (this implies choices described in the previous chapter, including the state variables themselves, their dimensions, and the limits of the system);

2- decide on a time frame, a time step, and an integration procedure;

3- devise equations that relate the state variables, the rates, and the parameters of the model among themselves;

4- run the model, and see the outputs as graphs or tables; and

5- check the program corresponding to the model which has been developed. These different stages are linked in different windows of the same STELLA® file.

\section{Exponential growth}

In the previous chapter, exponential growth was used as an example for integration, both analytical and numerical. Here, we shall first model exponential growth with the same hypotheses as in the previous chapter: (1) there are no limits to growth (i.e., unlimited supply of nutrients, no self-toxicity), and (2) the rate of growth is constant over time. 
One first has to use a state variable, the amount of bacteria, A. A state variable is represented by a box. The next component of the system is a flow of bacterial growth governed by a rate of growth, RA (the number of new bacteria formed per time step). This is represented under STELLA $®$ by a double arrow and a valve. The third element of the system is the relative rate of growth (the number of new bacteria formed per bacterium per time step), RRA. RRA is a parameter (in this example a fixed-value parameter) represented by a circle.

The system also involves relationships among some of its components, that is, flows of information. These relationships concern the effect of RRA on RA (i.e., we state that: 'RA depends on RRA'), and the effect of A on RA (we state that: 'RA also depends on A'). These relationships are represented by simple arrows.

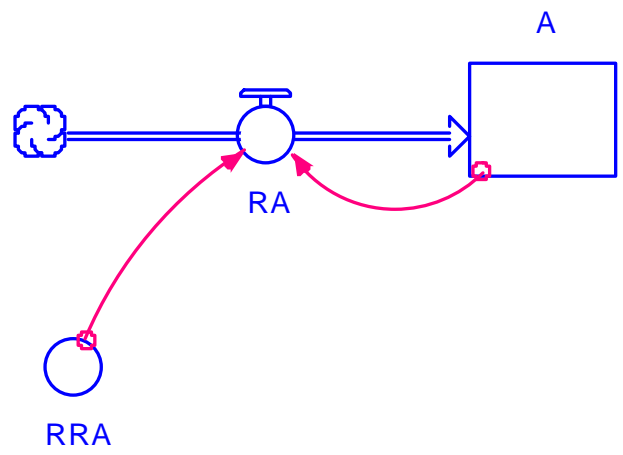

Figure 3.1. A flowchart representing exponential growth.

The flowchart is shown in Fig. 3.1. This very simple drawing therefore says that the amount of bacteria, A, grows with a rate RA, which depends on both the current number of bacteria A and a relative (or 'intrinsic') rate of increase, RRA.

The model corresponding to this diagram then needs documentation. Let us for instance assume that the initial number of bacteria is 1 (i.e., $\mathrm{A}_{0}=1$ ), and that the relative rate of bacterial increase is 0.3 , that is, that each bacterium produces 0.3 new bacterium per time step, $\Delta \mathrm{t}$.

The time characteristics of the system need then to be specified. We may, for instance assume that one is dealing with a $24-\mathrm{h}$ experiment, and that the bacterium population is monitored on an hourly basis. One would, then run the simulation with a time step of $\Delta \mathrm{t}=1 \mathrm{~h}$ over a period of time of $24 \mathrm{~h}$. 


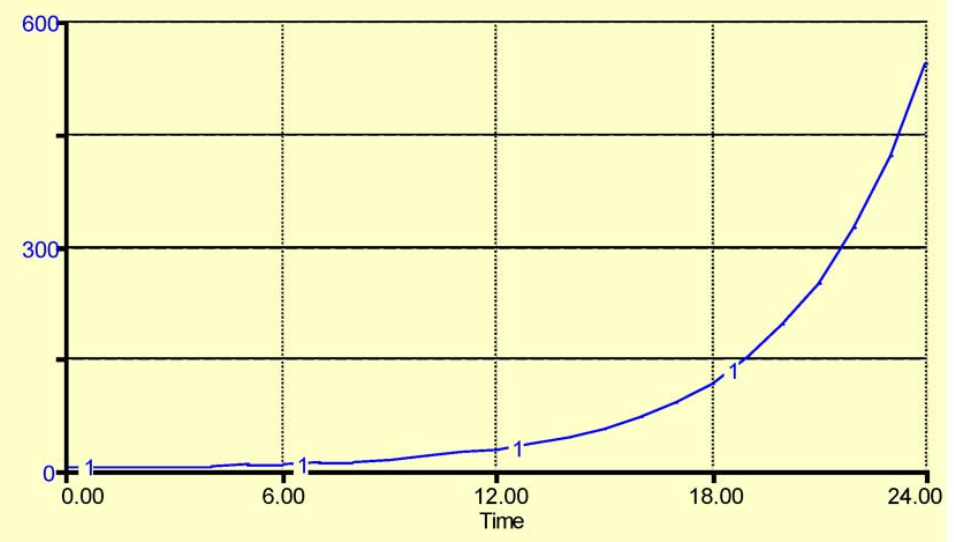

Figure 3.2. Simulated exponential growth. Horizontal axis: time (hours); vertical axis: number of bacteria.

The result is shown in Fig. 3.2, where $t$ is the abscissa and A is the ordinate, with the expected exponential number of bacteria increasing over time.

As a last step, we need to take a look at the program, written for STELLA ${ }^{\circledR}$, as follows:

$\mathrm{A}(\mathrm{t})=\mathrm{A}(\mathrm{t}-\mathrm{dt})+(\mathrm{RA}) * \mathrm{dt}$

INIT A $=1$

INFLOWS:

$$
\begin{aligned}
& \mathrm{RA}=\mathrm{A} * \mathrm{RRA} \\
& \mathrm{RRA}=0.3
\end{aligned}
$$

\section{Varying the parameter of exponential growth}

The previous paragraph reassures us that we indeed can easily develop a simulation model, even though it admittedly is a very simple one. One question which quickly may arise is whether and to what extent our model is sensitive to its parameter, RRA. To address this question, one can arbitrarily assign to RRA a series of different values, such as: $0.01,0.05,0.1$, 0.2 , and 0.3 .

The resulting graph is shown in Figure 3.3. As expected, any incremental increase in the relative rate of increase parameter corresponds to curves that strongly differ not in shapes, but in slopes (speeds) and maxima. This result shows how sensitive the model is to variations of RRA. 
This does not come as a surprise, of course, but further ensures us that the model does behave as exponential processes do.

Such a simple exercise (analyzing the effects of parameter change on the model output) actually is a sensitivity analysis, used in this case to check whether the program behaves as intended. Sensitivity analysis is a field of its own, with many applications, which we cannot address here.

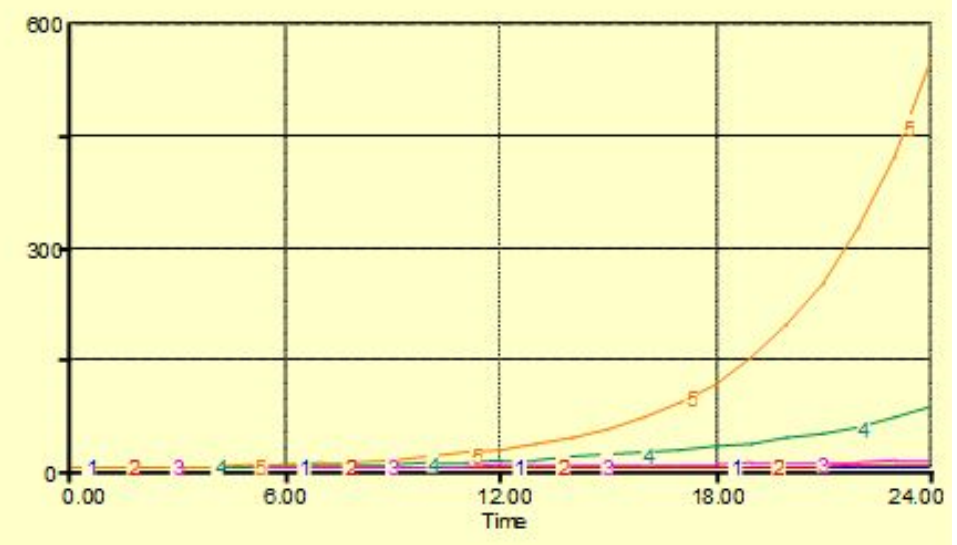

Figure 3.3. Simulated exponential growth with different values of the relative rate of increase, RRA. Curves 1 to 5 correspond to RRA values of $0.01,0.05,0.1,0.2$, and 0.3 , respectively. Horizontal axis: time (hours); vertical axis: number of bacteria.

Introducing a driving variable: exponential growth with varying relative rate

Until now, we have assumed that the relative rate of increase, RRA, of the exponential process is constant. In many cases, environmental conditions are such that processes involved in a system are influenced. Botanical epidemiology, for instance, provides a wide range of such external influence on the behavior of epidemics.

Still using our exercise exponential model, it is possible to explore ways to see how such external influences may be modeled. Let us for instance assume that RRA varies with temperature, such that, from experimental results, we have the following Table 3.1. 
Table 3.1. RRA values according to temperature

\begin{tabular}{|c|c|}
\hline Temperature $\left({ }^{\circ} \mathrm{C}\right)$ & RRA $\left(\mathrm{h}^{-1}\right)$ \\
\hline 0.00 & 0.00 \\
\hline 10.0 & 0.08 \\
\hline 20.0 & 0.16 \\
\hline 30.0 & 0.21 \\
\hline 40.0 & 0.24 \\
\hline 50.0 & 0.25 \\
\hline
\end{tabular}

This table indicates that at a temperature of $0^{\circ} \mathrm{C}, \mathrm{RRA}$ is 0 , then increases linearly, and then tapers off when temperature approaches $50^{\circ} \mathrm{C}$. Instead of a table, this information could be represented by a graph of RRA as a function of temperature. Between each (temperature, RRA) pair of data points, a segment would be drawn, which amounts to a linear interpolation. Many programming languages enable the computation of values that RRA would take according to temperature from such linear interpolations.

Furthermore, let us assume that the experiment was conducted under conditions such that the temperature would have been oscillating around a mean temperature of $20^{\circ} \mathrm{C}$ with an amplitude of $10^{\circ} \mathrm{C}$, which amounts to:

Temp $=$ TempMean + AmpTemp*sin $(2 * \pi *$ time/24)

where Temp is the running temperature, TempMean is the mean temperature, and AmpTemp is the amplitude about which the running temperature varies.

These new elements can be incorporated in the model by adding new components:

- a Temp variable which influences RRA, and

- two driving functions, TempMean and AmpTemp, which, in turn, makes Temp vary. The model diagram is represented in Fig. 3.4. 


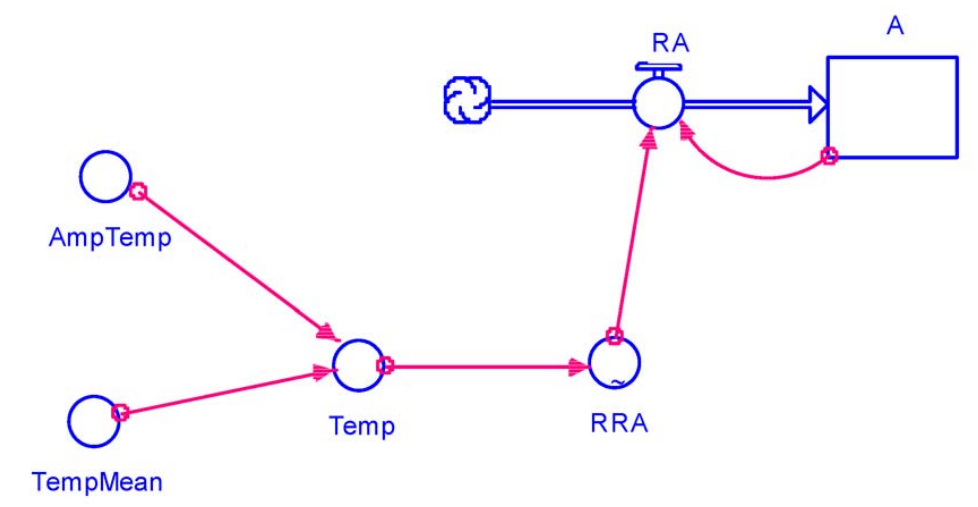

Figure 3.4. A flow chart representing exponential growth with temperature variation as a driving variable.

TempMean and AmpTemp are called driving functions (or driving variables), because they influence the system under consideration externally. If we were strictly using Forrester's symbols, they should be represented by segments with a dot at their center (see previous chapter) in order to indicate that they are different in nature from RRA, which is a parameter inherent to the model under consideration.

The question is whether large variations in RRA, as indicated by the effects of temperature on RRA in the previous table, could strongly influence the behavior of the system. Fig. 3.3 showed how strong the effect of RRA on the model's output can be, and thus one expects a strong response. Under the above assumptions on RRA's response to temperature, and temperature variation over time, one thus expects a strong change in the system's behavior. 


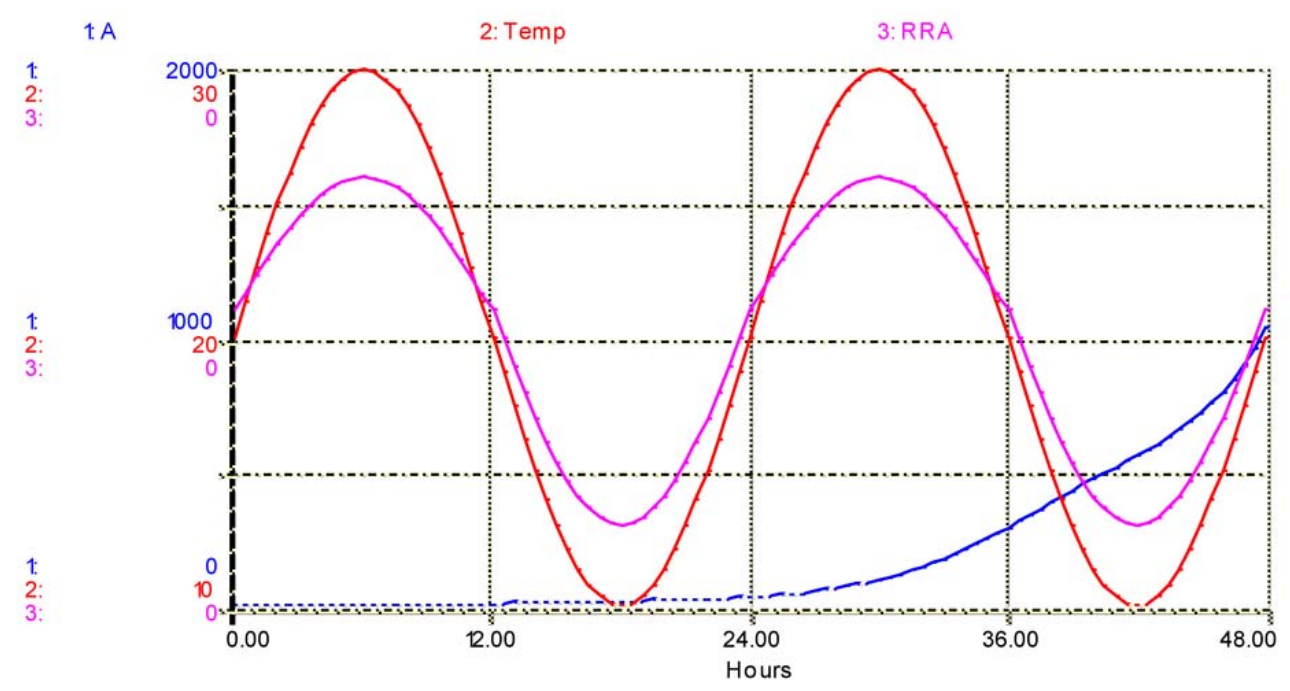

Figure 3.5. Simulation of bacterial numbers (A), temperature (Temp) and relative rate of bacterial increase (RRA). Horizontal axis: time (hours); vertical axis: number of bacteria (A), relative rate of increase of bacterial population (RRA), and temperature (Temp).

Contrary to our initial intuitive reasoning, Fig. 3.5 indicates that the shape of the bacterial population curve is barely influenced by changing temperatures: it still remains an exponential process. Yet, the variations over time of temperature, and of RRA over temperature, are showing the expected sinusoid shapes.

Of course, the values of Table 3.1 were chosen on purpose for this example. We could also have made the amplitude in temperature variation much broader but this would not alter the outcome: what was first designed as an exponential process remains.

\section{The limits to growth - a summarized phytopathological perspective}

In a famous report entitled 'The Limits to Growth' to the Club of Rome, Meadows et al. forwarded a grave warning in 1972 to the international community: unlimited growth (as most conventional economists and demographists see it) cannot possibly take place, disregarding the global population growth and the limited resources of the biosphere. This was followed by a series of sequels, including 'Beyond the Limits - Global Collapse or a Sustainable Future' in 1992 (which actually makes extensive use of systems concepts, simulation modeling, and of the STELLA® program), and 'World on the Edge - How to Prevent Environmental and Economic Collapse' in 2011. These warnings have repeatedly been dismissed as simplistic, biased, and 
Malthusian, because they did not factor in the human ingenuity to solve problems as they emerge. G. Hardin (1968) however made a very strong case in his article on the 'Tragedy of the Commons' in highlighting this particular and (usually extremely important) class of problems which can be called "no technical solution problems".

From a modeler's standpoint, the above example illustrates one of the many uses of simulation modeling. Simulation models enable researchers to conduct (simulation) experiments on systems in which no material experiments could possibly be conducted. There is only one biosphere, and experiments where the rate of, for example, population growth, the amount of available water, the fraction of human beings living in cities, and the amount of food produced per capita would all be varied, are simply unthinkable. Carefully designed models, hypotheses (that is to say, building scenarios), and simulations, as well as cautious interpretations of simulation results, allow us to precisely do that.

Plant disease epidemiologists are confronted with limited growth on a routine basis: a crop has only a limited number of sites (e.g., individual plants, leaves, or fragments of roots, say, per square meter) which may become infected. Once infected, such sites cannot be infected again, and the pathogen is confronted with a limited carrying capacity at each point of time. Epidemics do occur in natural ecosystems too, which are characterized by one among many differences: whereas a crop is to be seen as a cohort of individuals of approximately the same physiological age, and very often, with a similar genetic make-up, host plants in natural ecosystems do not have the same physiological age, and differ genetically. Nevertheless, in both kinds of systems, a limit to (disease) growth exists.

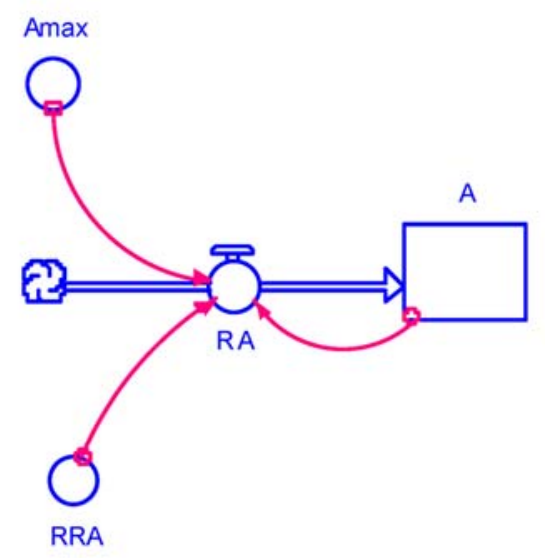

Figure 3.6. Flowchart representing exponential growth with limited growth. 
For now, let us return to our simple bacterial model. One important change in the model structure is to consider that bacteria are not growing in an unlimited volume, with unlimited supply of nutrient. We then would introduce the notion of carrying capacity in the model, in this case, represented by the maximum number of bacteria the system might possibly contain. The starting value of the bacterial population was set at 1 (this "1" might represent $1 \times 10^{6}$ bacteria). Let us now assume that the maximum number of bacteria in the system could only be 100 (or $100 \times 10^{6}$ bacteria). The model structure then becomes as shown in Fig. 3.6.

Introducing Amax slightly changes the program, as the equation for RA now has to account for Amax, namely, RA is not only function of RRA but is also a (multiplying) function of the distance to which the current bacterial population is far away from reaching its maximum possible value, which we write: (1 - (A/Amax)). This term will be referred to the 'correction factor' in the following chapter. The corresponding modeling program becomes:

$\mathrm{A}(\mathrm{t})=\mathrm{A}(\mathrm{t}-\mathrm{dt})+(\mathrm{RA}) * \mathrm{dt}$

INIT A $=1$

INFLOWS:

$$
\begin{aligned}
& \mathrm{RA}=\mathrm{A} * \mathrm{RRA} *(1-(\mathrm{A} / \mathrm{Amax})) \\
& \mathrm{Amax}=100 \\
& \mathrm{RRA}=0.3
\end{aligned}
$$

The resulting bacterial dynamics is shown in Fig. 3.7 with a typical sigmoid curve, with which ecologists, microbiologists, and plant pathologists, are so familiar.

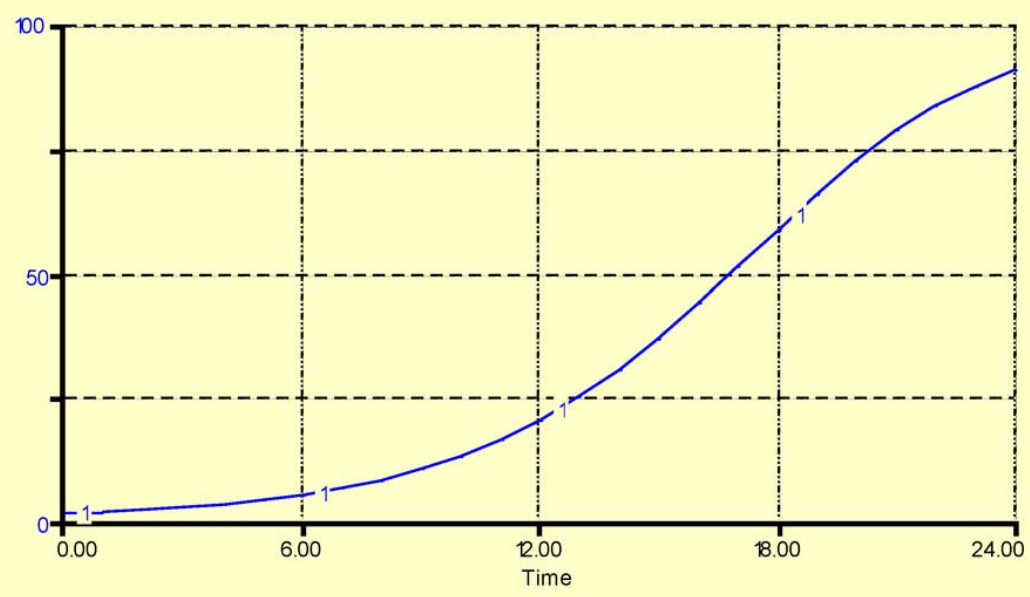

Figure 3.7. Simulated exponential growth with limited growth. Horizontal axis: time (hours); vertical axis: number of bacteria. 


\section{Summary}

This chapter has introduced some key elements of model development:

- Steps in developing a simulation model including: (1) drawing the relationships representing the model structure; (2) choosing a time frame, a time step, and an integration procedure; (3) running the model, and see the outputs as graphs or tables; and (4) checking the program corresponding to the model which has been developed.

- A very simple system of bacterial growth has been used to illustrate the concepts of rate of growth of a state variable and of relative rate of growth.

- Changes in the value of the relative rate of growth (RRA) have profound consequences in the numerical outcomes of simulations - but not in the shapes of the simulated curves. Varying RRA and assessing the behavior of the system may be seen as a preliminary, qualitative, example of sensitivity analysis.

- The notion of driving function (variable) - of a variable which lies outside the boundaries of the considered system, but may influence it - is introduced. In the bacterial growth example, where the effect of oscillating temperature on RRA is used. Although temperature variation does affect the running value of the rate of growth (RA), it does not truly affect the overall behavior of the system: an exponential process is retained.

- The notions of limited growth and carrying capacity of a system are introduced. This has dramatic consequences on the behavior of the system, and yields a sigmoid growth.

\section{References}

Kranz, J. 1988. Measuring plant disease. Pages 35-49 in: Experimental Techniques in Plant Disease Epidemiology. J. Kranz and J. Rotem, eds. Springer Verlag. Berlin, Heidelberg, New York.

Brown, L.R. 2011. World on the Edge - How to Prevent Environmental and Economic Collapse.

Earth Policy Institute. W.W. Norton \& Company, New York, London.

Forrester, J.W. 1961. Industrial Dynamics. M.I.T. Press, Cambridge (Mass.).

Hardin, G. 1968. The tragedy of the commons. Science 162:1243-1248.

Leffelaar, P.A., ed. 1993. On Systems Analysis and Simulation of Ecological Processes. Current Issues in Production Ecology. Kluwer Academic Publishers, Dordrecht.

Meadows, D.H., Meadows, D. L., Randers, J., and Behrens, W.W. III 1972. The Limits to Growth: A Report to The Club of Rome. 
Meadows, D.H., Meadows, D. L., and Randers, J. 1992. Beyond the Limits - Global Collapse or a Sustainable Future. Earthscan, London.

Penning de Vries, F.W.T., and Van Laar, H.H., eds. 1982. Simulation of Plant Growth and Crop

Production. Pudoc, Wageningen.

de Wit, C.T., and Goudriaan, J.G. 1978. Simulation of Ecological Processes. Pudoc, Wageningen.

\section{Suggested reading}

Case, J.T. 2000. An Illustrated Guide to Theoretical Ecology, Oxford University Press, New

York.

Exercises and questions

1. The rate of growth in case of the exponential growth of a variable $\mathrm{N}$ is
a. $\quad \mathrm{RG}=\mathrm{r} \times \mathrm{N} \times(1-(\mathrm{N} / \mathrm{Nmax}))$
b. $\mathrm{RG}=\mathrm{r} \times \mathrm{N}^{2}$
c. $\mathrm{RG}=\mathrm{r} \times \mathrm{N}^{-1}$
d. $R G=r \times N$

2. Increasing the relative rate of growth in case of the exponential growth of a variable $\mathrm{N}$
a. Does not affect the dynamics of $\mathrm{N}$
b. Increases the rate of growth
c. Increases the final value of $\mathrm{N}$
d. Decreases the rate of growth

3. A driving function
a. Can be constant over time
b. Corresponds to processes simulated within the system
c. Can vary over time
d. Does not affect the system modeled

4. The rate of growth in case of the exponential growth of a variable $\mathrm{N}$ with a carrying capacity Nmax is 

a. $\mathrm{RG}=\mathrm{r} \times \mathrm{N} \times(1-(\mathrm{N} / \mathrm{Nmax}))$
b. $\mathrm{RG}=\mathrm{r} \times \mathrm{N} \times(1-\mathrm{N})$
c. $\mathrm{RG}=\mathrm{r} \times \mathrm{N}^{-1}$
d. $\mathrm{RG}=\mathrm{r} \times \mathrm{N} \times(1+(\mathrm{N} / \mathrm{Nmax})$

5. The dimension of the relative rate of growth of a variable $\mathrm{N}$ having an exponential growth is
a. $\left[\mathrm{N} . \mathrm{T}^{-1}\right]$
b. $\left[N \cdot N^{-1} \cdot T^{-1}\right]$
c. $\left[\mathrm{N} . \mathrm{N}^{-1}\right]$
d. does not change if the equation of the rate of growth includes a carrying capacity

Answers to exercises and questions

1. $\mathrm{d}: \mathrm{RG}=\mathrm{r} \times \mathrm{N}$

2. b: Increases the rate of growth, and c: Increases the final value of N.

3. a: Can be constant over time, and c: Can vary over time.

4. $\mathrm{a}: \mathrm{RG}=\mathrm{r} \times \mathrm{N} \times(1-(\mathrm{N} / \mathrm{Nmax}))$

5. b: $\left[\mathrm{N} . \mathrm{N}^{-1}\right]$, and d: does not change if the equation of the rate of growth includes a carrying capacity 


\section{Chapter 4. A Preliminary Epidemiological Example}

An epidemic may be seen as a whole, an entity to be studied, which we can refer to as a process. This process results from underlying mechanisms, which we can refer to as subprocesses. Therefore, the building blocks of plant disease epidemics, as processes, consist of subprocesses. For instance, for an aerially dispersed disease, one may consider the following subprocesses: propagule production, propagule liberation, propagule transport, propagule deposition, infection, latency period, and infectious period. The process itself thus consists of linked subprocesses, monocycle components, which have been collectively called the 'infection chain' by $\mathrm{J}$. Kranz (1974). It is the concatenation of infection chains that leads to an epidemic. This chapter deals with the modeling of an epidemic, as a process, on the basis of knowledge of processes at the next lower level of integration, that is to say, the monocycle components.

One could also model one of the sub-processes, such as propagule formation (for example for an asexually reproducing fungus: conidiophore initiation, conidiophore elongation, conidiophore branching, spore initiation, spore maturation). One could also consider the system where epidemics are sub-processes of a higher-scale process called polyetic epidemics. In this case, it is the concatenation of successive individual epidemics, as lower levels of integration, which results in polyetic epidemics occurring over many successive seasons, as upper levels of integration. The former case might be of interest to study, e.g., how genes involved in spore production in a pathogen influence the dynamics of sporulation, which in turn can have applications in understanding one of the biological bases of host plant resistance. The latter case is of interest in understanding how, and to what extent, successive epidemics are related (especially via the primary inoculum), i.e., what is the basis of the carry-over of epidemics across time, and this can have applications in disease management over seasons.

Biology, in general, is concerned with hierarchies of processes. It is up to the scientist to choose which level of this hierarchy should be the focus of an investigation. This chapter focuses on epidemics. Epidemics, as biological phenomena, can be decomposed in sub-processes, which in turn can be decomposed in sub-sub-processes. Systems analysis, in turn, using (among other tools) simulation modeling, enables one to investigate and understand the behavior of one level of a system's hierarchy, making use of knowledge acquired on the next-lower level of integration within a biological hierarchy. 
This chapter concentrates on one of today's principal structures in plant disease epidemiology. This structure remains an important, quite current (e.g., Cunniffe et al., 2012; Van den Bosch et al., 2008; Segarra et al., 2001), field of investigation in its own, although having been published by Zadoks in 1971 (Zadoks, 1971). The model elaborates on the foundations developed by Van der Plank (1963), with the concepts of:

- infection,

- latency period, and

- infectious period,

which are captured by the differential-delay equation:

$d x t / d t=\mathrm{R}_{c}\left(x_{t-p}-x_{t-i-p}\right)\left(1-x_{t}\right)$ (equation 8.3, p. 100, Van der Plank, 1963),

where $x_{t}$ is the amount of disease at time $t, \mathrm{R}_{c}$ is the basic infection rate corrected for removals, $p$ is the latency period duration, and $i$ is the infectious period duration.

\section{Components of a preliminary epidemiological model}

The simulation model developed by Zadoks (1971) provides a numerical integration of Van der Plank's seminal equation of botanical epidemiology. As in the earlier example, we need to first define the system under consideration and its components.

The system under consideration is a $1-\mathrm{m}^{2}$ crop area surrounded by similar crop areas. This crop consists of sites, which may be healthy (HSites), or infected. Sites that have been infected can be partitioned in three, non-overlapping, categories: sites that have been infected but are not yet infectious, and therefore are latent (LatS), sites that are infectious and are therefore generating propagules (InfS), and sites that are no longer infectious and thus are removed from the infectious process (RemS). The notion of site, therefore, refers to those plant tissues that can sustain a given infection and give rise to new ones. Sites, therefore, will not be the same depending on the pathosystem: for example, in the case of systemic diseases, a site will refer to an entire plant unit, whereas for leaf- (or, e.g., fruit-) spotting diseases, a site will refer to a (potential or existing) lesion.

In the following, let us concentrate on the case of a disease that is aerially dispersed and causes lesions on leaves. This is an important consideration, because it determines the nature of the state variables that are of primary concern in the considered system. In this case, we are thus dealing with a population of sites, whose transitions from healthy, to latent, to infectious, and to removed, are dynamically tracked. 
In addition to the choice of system's limits, let us further assume that the time step is one day. Many epidemiological models use such a time step, in large part because, for most weather data sets available, the climatic day starts at about 7 a.m., and ends the following day at the same time. In the intervening time - a full day - many epidemiological events happen in our system: for instance, spores are produced, liberated, and deposited, and infections take place.

Epidemiologists are well aware that these events do depend on environmental factors (the weather, but possibly the physiological status of the host plants, too) that vary with a much smaller time constant (e.g., a wind gust in the canopy, a short shower, or the progressive dry-off of moist leaves). However, these factors may only influence mechanisms that are themselves subprocesses of a sub-process (i.e., the infection process) at hand. In other words, these factors influence sub-sub-processes, whereas our endeavor is to numerically integrate sub-processes and quantify their consequences at the process level. Yet, one must devise a modeling approach that enables to consider sub-processes themselves, without ignoring the possible importance of processes at a lower level of integration. This is directly related to the time constant we assume the system has. We shall return to this important consideration at the end of this chapter.

\section{Main equations of the model}

Since the sites are non-overlapping categories, one may write:

$\mathrm{ACI}=\mathrm{LatS}+\mathrm{InfS}+\mathrm{RemS}$,

where ACI is the total number of infected sites. However, only infectious sites (InfS) can produce propagules which can lead to additional InfS, while infection can only take place on sites that are still healthy at one point of time. Thus, it is convenient to calculate:

$\mathrm{CORF}=1-(\mathrm{ACI} /(\mathrm{ACI}+\mathrm{HSites}))$, where CORF, a 'correction factor' for site availability, represents the proportion of healthy sites that are still available for infection, or the probability of a site to be healthy (CORF is strictly comprised between 0 and 1). Being a ratio of quantities with the same dimensions ([N]), the dimension of CORF is: $\left[\mathrm{N} \cdot \mathrm{N}^{-1}\right] \equiv[1]$.

In the course of any day, propagules are being produced, released, transported, and deposited, and infection may take place on healthy sites. The rate of infection can be expressed as:

INFECTION $=$ DMFR $*$ CORF*InfS 
In this equation, INFECTION is a rate, and therefore has the dimension of a speed: [N.T ${ }^{-1}$. InfS is the number of infectious sites at a given point of time $([\mathrm{N}]), \mathrm{CORF}$ is the correction factor, and DMFR is a daily multiplication factor (Daily Multiplication FactoR). DMFR is the number of daily new infections originating from existing, infectious lesions, or, the daily number of daughter-lesions per mother-lesion, with dimension $\left[N \cdot N^{-1} \cdot T^{-1}\right]$. In essence, DMFR is analogous to the intrinsic rate of bacterial population increase of the previous chapter. From an epidemiological standpoint, DMFR, in the mechanistic wording used in this model, exactly corresponds to $\mathrm{R}_{c}$, the basic infection rate corrected by removals used by Van der Plank (1963). The dimension of INFECTION is therefore $\left[\mathrm{N} \cdot \mathrm{N}^{-1} \cdot \mathrm{T}^{-1}\right] \cdot[1] \cdot[\mathrm{N}] \equiv\left[\mathrm{N} \cdot \mathrm{T}^{-1}\right]$. It has the dimension of a rate, that is, of the speed of a process.

Note that the INFECTION equation also collapses a number of sub-sub-processes together, some of which were mentioned above. In other words, with respect to the daily rate of infection, all of which happens in any given day is summarized in the equation for INFECTION. This, of course, is a very important simplification, which we make in order to adhere to the above principle of systems analysis: explaining a process from its immediately related sub-processes. This simplification can, however, be further documented while remaining at the same levels of integration. This will be revisited later on.

Additional elements need to be documented in the model structure. Both the latency period and the infectious period are important phases of the disease monocycle. Sites that are in both the latent and the infectious stages correspond to two state variables. These state variables, however, are of a particular type, because sites remain in these states for specified (and epidemiologically important) durations. Such delays in a given state are called residence times. Thus, we want to assign a residence time in these two states, $p$ days in the latent, and $i$ days in the infectious stage. These have been called 'boxcar trains' (to reflect a series of boxes through which each individual progresses; Penning de Vries and Van Laar, 1982), or, using the terminology used in STELLA®, 'conveyors'. We need to decide what these residence times are. Let us, for a start, assume that $p=6$ days and $i=10$ days.

Let us also further assume for the sake of simplicity that values for $p$ and $i$ are fixed throughout the duration of an epidemic. Epidemiologists know that this is a strong simplification: for instance, as plants become older both $p$ and $i$ may vary, expressing increasing, or decreasing, resistance with development. Both parameters are also bound to change during the course of an epidemic with weather variables. Simulation modeling (and software such as STELLA® in 
particular) enables one to incorporate daily changes in values of $p$ and $i$ from driving variables, such as the crop development stage or varying temperature. For now, because we want to first develop a simple model structure, let us nevertheless assume that both parameters do not change. Let us further assume that, as the epidemic starts, both state variables contain no individuals, that is, that there are no infected sites in the latent or the infectious stages.

\section{Initializing the model}

A number of statements need to be made in order to run the model. First one needs to specify the population size of the host. Let us assume that the initial number of healthy sites (HSites) is 100,000. Let us further assume that the duration of an epidemic is 100 days.

Next, we need to define a value for the daily multiplication factor, DMFR. Let us, for a start, assume that DMFR $=0.3$. A value of 0.3 for DMFR means that every day, a (mother) infectious site (InfS) can potentially give rise to 0.3 (daughter) infected site through the INFECTION rate. 'Potentially' implies here that there is enough 'space' for $0.3 * \operatorname{InfS}$ new infections to take place, that is, that propagules will reach healthy sites (HSites). In so doing, we only express the underlying hypotheses of Van der Plank's (1963) equation. We know that there are many constraints on the occurrence of infection besides the availability of healthy sites. We shall re-visit this key assumption at the very end of this chapter.

Another element concerns the initialization of the epidemic. There are several ways to do so. One approach could be to place infected sites in the latent stage as a starting point. Instead, because we want to be able to vary the date at which the epidemic starts, let us create an INOCPRIM parameter representing the amount of primary inoculum, which becomes active at a chosen point of time in the course of the growing season, and let us create a connected DAY parameter, which simply tracks time in the model (note that under STELLA®), 'TIME' represents the elapsing computing time). Let us further decide for now that INOCPRIM generates a single influx of new infections through INFECTION. We thus create a starting device, written as:

INFECTION $=($ DMFR $*$ CORF $*$ InfS $)+$ INOCPRIM

$\mathrm{DAY}=\mathrm{TIME}$

INOCPRIM = IF (DAY=1) THEN 100 ELSE 0

which states that at a given day (here, day 1), there is a single, one-day, influx of active primary inoculum (INOCPRIM) resulting in 100 sites becoming infected (latent). 


\section{Drawing the model's flow chart}

Our model flow chart is shown in Fig. 4.1, and depicts the different state variables and parameters, as well as their relationships. Note that the state variables for latent and infectious sites are shown as conveyors, and also the series of links determining the rate of infection (INFECTION), which reflect the series of assumptions made on state variables and parameters that determine its daily value.

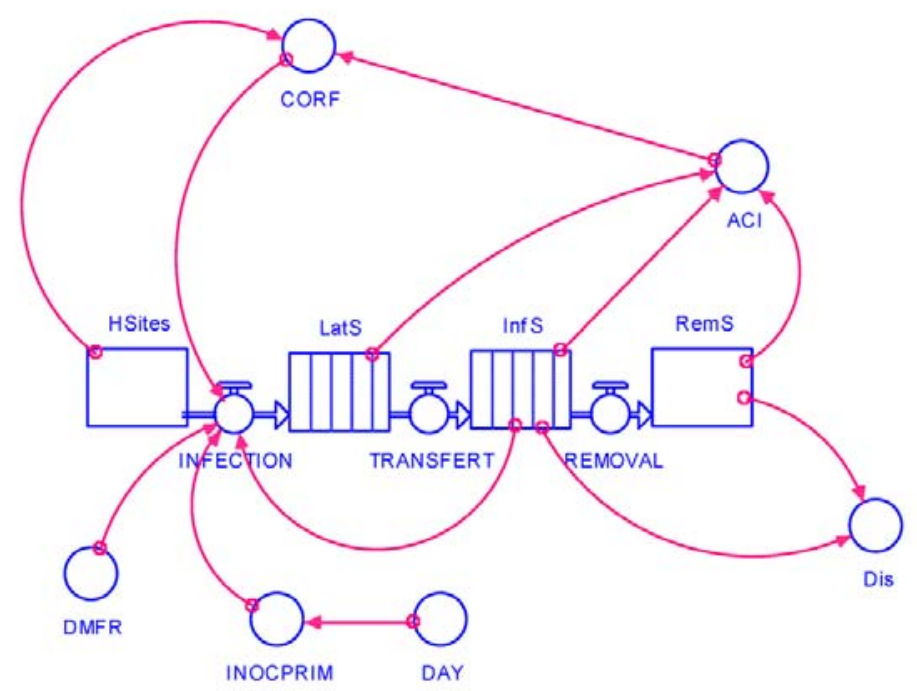

Figure 4.1. Flowchart of a preliminary epidemiological model for an aerially dispersed pathogen. HSites: healthy sites; LatS: latent sites; InfS: infectious sites; RemS: removed sites; Dis: (visibly) diseased sites; ACI: accumulated infected sites; CORF: correction factor for site infection; DMFR: daily multiplication factor; INOCPRIM: primary inoculum; DAY: running day. See Figure 2.1 and Table 2.1 for the meaning of symbols.

\section{Model verification: a first run}

A first stage in model evaluation consists of checking whether the model's program executes the intended instructions as originally designed (Penning de Vries and Van Laar, 1982). Such a task is easy in the case of this preliminary epidemiological model, and even easier in the case of the bacterial population model discussed in the previous chapter. Fig. 4.2 gives a graphical output of the dynamics of an epidemic. We can make the following remarks:

- the amount of visibly infected sites (Dis = InfS+RemS; curve 5) increases in a sigmoid pattern, as the stock of healthy sites is being depleted, and the effect of CORF on INFECTION comes into effect, slowing down the speed of the epidemic; 
- the amount of latent lesions (curve 3) progressively increases and then decreases, representing, in many ways, the slope of the disease (Dis) progress curve;

- the amount of infectious sites (curve 2) follows the same pattern as the amount of latent lesions, with a delay of about 6 days, that is, as expected, about $p$;

- removed sites (curve 4) accumulate regularly, as infected sites exit the latent and infectious stages.

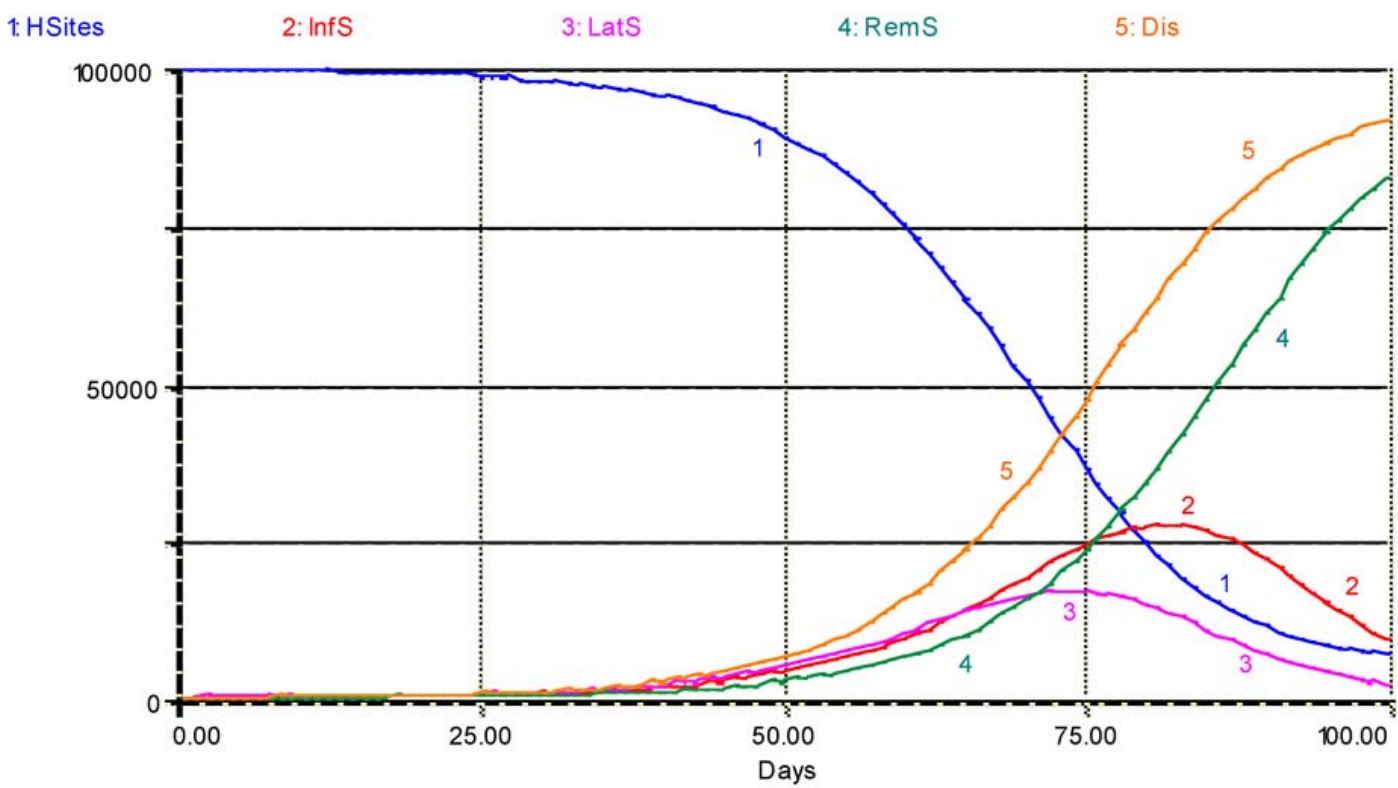

Figure 4.2. First output of a preliminary simulation: healthy, latent, infectious, removed, and visibly diseased sites. The values of parameters used are DMFR $=0.3$ lesions.lesion1.day- $1, \mathrm{p}=6$ days, $\mathrm{i}=10$ days, and date of onset is 1 . 1 : healthy sites; 2 : infectious sites; 3: latent sites; 4: sites removed from the epidemiological process; 5: accumulated visibly diseased sites (infectious and removed). Horizontal axis: time (days); vertical axis: numbers of sites.

The overall behavior of the model is, therefore, as expected and shows the patterns of disease progress described in so many reports. It does, therefore, conform to the expected. This model provides a visual and quantitative solution to the equation developed by Van der Plank some 50 years ago (simulation outputs could also be displayed in a tabular manner, which is not shown here).

A key element that simulation modeling brings about is the possibility to see what is not visible: Fig. 4.2 displays the dynamics of latent lesions, which, of course, would be impossible to 
monitor in the field. This same remark applies to removed and infectious sites which, in nearly all cases would be impossible to tell apart even for the most experienced field pathologist. Simulation modeling, therefore, allows visualization of the behavior not only of the process, but of the sub-processes considered.

\section{Exploring the model's behavior}

The effects of variations of a series of parameters in the model are shown in Fig. 4.3. This sensitivity analysis can be summarized as follows: (1) increasing values of DMFR from 0.01 to 0.5 lesion $\cdot$ lesion $\cdot$ day $^{-1}$ strongly increases the speed of epidemics (Fig. 4.3a); (2) increasing values of $p$ from 1 to 12 days strongly suppresses epidemics (Fig. 4.3b); (3) increasing values of $i$ from 1 to 20 days strongly increases the final level of disease (Fig. 4.3c); and (4) delaying the epidemic from 1 to 30 days strongly reduces the final level of disease as well (Fig. 4.3d).
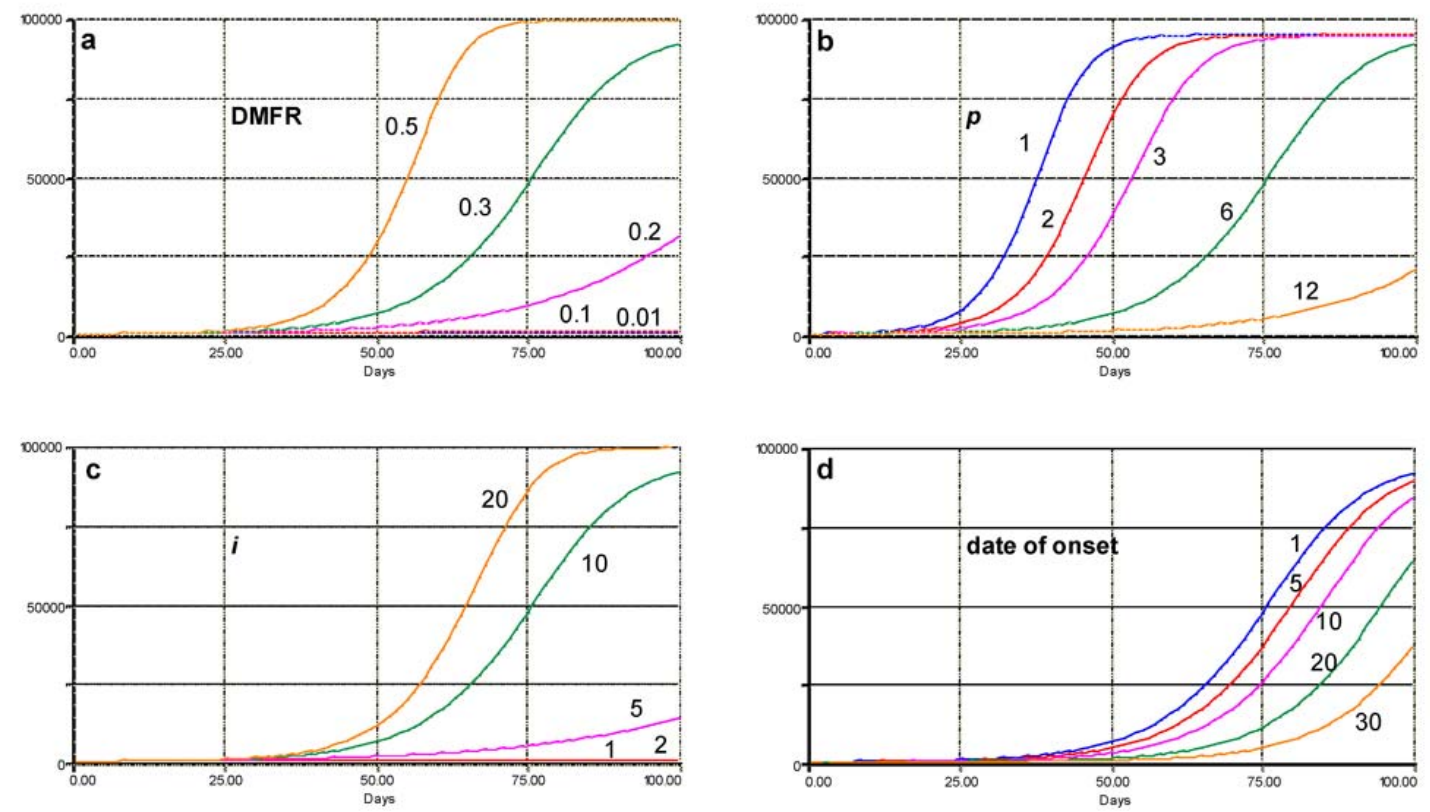

Figure 4.3. Sensitivity analyses of variations in DMFR, latency period, infectious period, and date of epidemic onset. The simulated number of diseased sites (infectious and removed) is displayed in all figures. a: effects of varying DMFR values; b: effects of varying values of latency period; c: effects of varying values of infectious period; $d$ : effects of varying dates of onset values. The default parameters values are DMFR $=0.3$ lesions.lesion-1.day-1, $\mathrm{p}=6$ days, $\mathrm{i}=10$ days, and date of onset is 1 . Values of parameters which are varied are indicated on the simulated curves. Horizontal axis: time (days); vertical axis: numbers of sites. 
These are well-known effects of key epidemiological parameters (Van der Plank, 1963; Zadoks, 1971; Zadoks and Schein, 1979) on plant disease epidemics involving a large number of overlapping and concatenated infection chains. This further indicates that the model structure we developed conforms to what has become to be known as classical epidemiological theory.

Another outcome of these runs is, simply, that simulation modeling allows one to "see" Kranz's (1974) infection chain in action in a polycyclic process. Often, changes in value of a parameter has little immediate effect, but, as disease cycles overlap in the course of an unfolding epidemic (Teng, 1983), the compounding effect becomes stronger, sometimes with dramatic consequences.

\section{Revisiting hypotheses}

The development of this model structure, despite its conforming to known epidemiological principles, is based on a number of hypotheses. Exploring these hypotheses are grounds for very current and active research (e.g., Segarra et al., 2001; Cunniffe et al., 2012). We address some of these hypotheses briefly, with a focus on assessing the validity of the model structure.

A first hypothesis concerns the area of the system considered and its boundaries. The system under consideration here consists of a $1-\mathrm{m}^{2}$ crop area surrounded by similar systems. A 1$\mathrm{m}^{2}$ crop area may, for instance, be relevant for a cereal or a legume crop. One would obviously have to increase this size when considering most perennials or semi-perennial crops. The assumption also implies that these boundaries allow fluxes of propagules to enter and exit the system in a steady-state. What is implied by 'similar crop areas' surrounding our system is that the amount of disease is the same in surrounding areas. It also implies that the crop structure does not vary greatly, so that the microclimatic conditions would, in our system, be representative of the conditions that prevail in neighboring, equivalent systems. In this preliminary epidemiological example, the system under consideration is kept as simple as possible; the limited size of the system, the variability of the host population size over time, the consequences the host population size may have on microclimate or disease spread, for instance, are disregarded for the sake of simplicity. The hypothesis of a limited system surrounded by similar systems with which it is in a dynamic equilibrium (e.g., flows of propagules, heat, water vapor) is often referred as the 'mean field' hypothesis. Although the model may have usefulness in its ability to understand and 
compare quantitatively sub-processes (components of the epidemiological monocycle) in determining the outcome of processes (epidemics), the 'mean field' is a very strong hypothesis which must lead to cautious interpretation of results.

Another hypothesis is that we refer here to aerially-dispersed (fungal or bacterial) diseases. This has two implications; one is that the model structure deals with processes that are typical of aerial dispersal. The other implication is that the population of sites which is considered consists of fractions of leaf tissues that potentially may become lesions. Regarding this second implication, one should note that the same model structure can effectively be used to address sites of other dimensions in a host population hierarchy, from fractions of leaves, to leaves, to shoots, or entire plants (Savary et al., 2012).

A further assumption in the model is that we chose to use a one-day time step. This leads us to collapse the liberation-transport-deposition-infection process into a single rate, INFECTION, governed by DMFR and CORF. One must question whether DMFR could possibly be kept constant throughout a cropping season. DMFR can actually be made dependent on time, whether because weather varies (and thus affects dispersal and infection sub-sub-processes) or host tissues become less and less susceptible over time as they age. In the case of weather variation, driving functions (e.g., air temperature) could be entered in the model as tabulated values using STELLA $®$ and Excel®. Simple assumptions can also be made. In the case of ageing, and increasingly resistant, tissues, let us for instance assume that DMFR decreases exponentially as:

$$
\mathrm{DMFR}=0.3 * \operatorname{EXP}\left(-\mathrm{k}^{*} \mathrm{DAY}\right)
$$

where $\mathrm{k}$ is a positive extinction coefficient. The outputs of three values for $\mathrm{k}(0$ : no resistance, 0.005: moderate resistance, and 0.01: strong resistance) are plotted in Fig. 4.4, showing again how DMFR strongly influences the behavior of the model. 


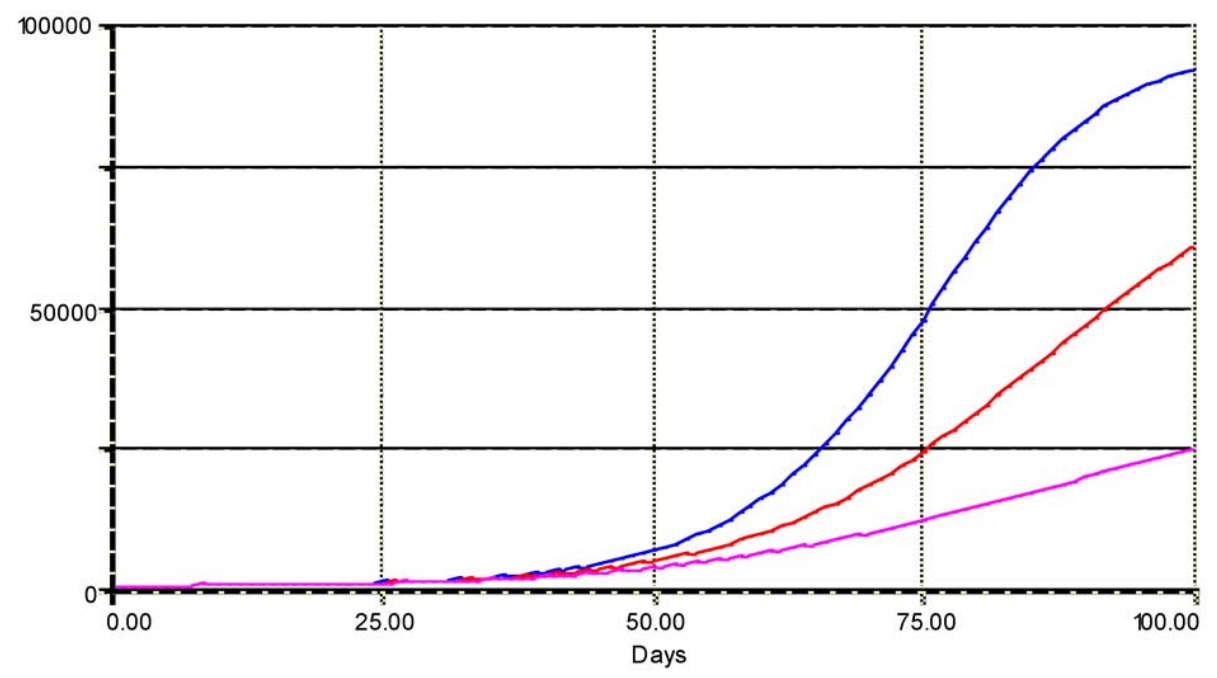

Figure 4.4. Simulated disease progress with exponentially decreasing values of DMFR. The simulated number of diseased sites (infectious and removed) is displayed. Upper, medium, and lower curves are simulated with an extinction coefficient over time of 0 , 0.01 , and 0.005 , respectively. See text for details. Horizontal axis: time (days); vertical axis: numbers of sites.

Similar approaches could be used if one wanted to address other sub-sub-processes, such as spore germination, germ tube elongation, penetration, and establishment of host-pathogen interaction, leading to actual infection.

Conversely, one can hardly imagine that, in the real world, both the latency, $p$, and infectious, $i$, periods would remain constant throughout a 100-day epidemic (e.g., Cunniffe et al., 2012). The current model architecture is flexible enough to incorporate such changes. For instance, the TRANSFERT rate between the two boxcars representing the latency and the infectious periods can be made also a function of temperature (in addition to the inherent characteristics allowing such state variables to vary properly; de Wit and Goudriaan, 1978). Let us assume that, for instance, during the 100 days, the environmental conditions (say, an increasing temperature) translate into having increasing values of $p$. This can be simulated, and the simulation outputs are shown in Fig. 4.5. The increasing value of $p$ leads, as expected, to a slower disease progress, and to a lower final disease intensity. 


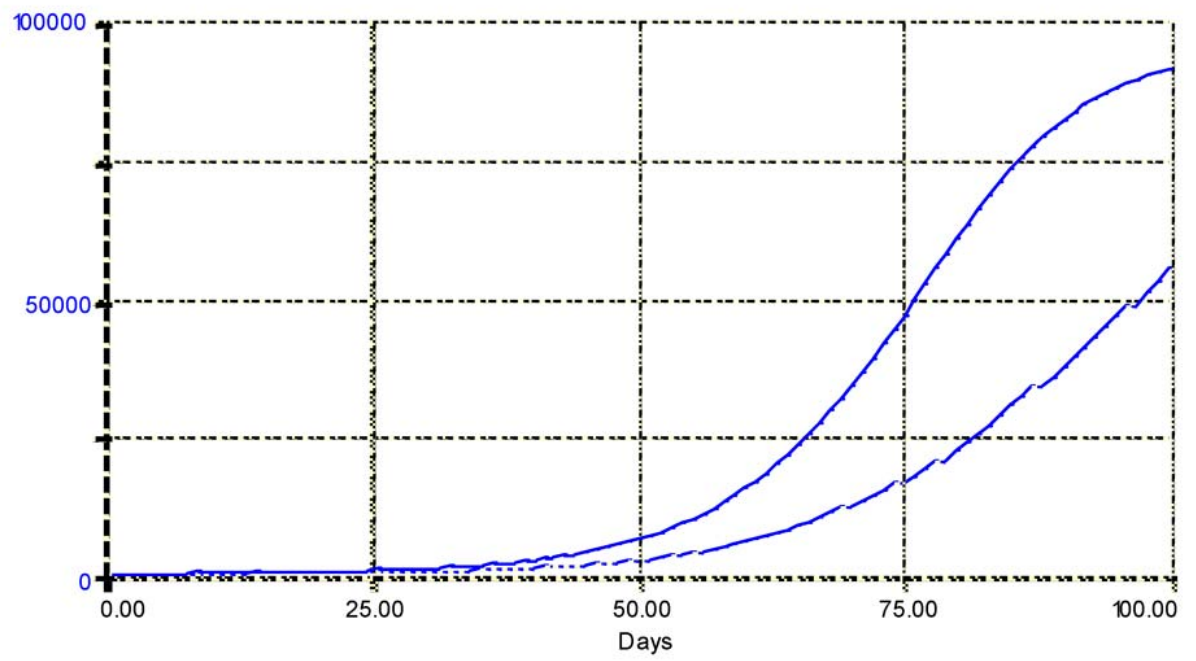

Figure 4.5. Simulated disease progress curves with constant (upper curve) or varying (lower curve) temperature influencing the latency period duration, p. The lower curve is associated with days when temperature may be high, leading to an increase in $\mathrm{p}$. The simulated number of diseased sites (infectious and removed) is displayed. Horizontal axis: time (days); vertical axis: numbers of sites.

The preliminary model in a broader context (1): the basic infection rate corrected for removals

Let us look backward, and consider again Van der Plank's differential-delay equation: $d x_{t} / d t=\mathrm{R}_{\mathrm{c}}\left(x_{t-p}-x_{t-i-p}\right)\left(1-x_{t}\right)$, and let us remember that the preliminary simulation model discussed here provides a numerical integration of this equation. Two key differences between analytical and numerical integration can be highlighted. While analytically solved equations produce exact solutions, numerical integration over a chosen time interval, $\Delta \mathrm{t}$, only generates numerical estimates, which however can easily be derived, even when parameters vary over time. Simulation modeling provides an easy way to integrate Van der Plank's equation, which is quite complicated analytically (Madden et al., 2007).

\section{The preliminary model in a broader context (2): the basic reproduction number}

The total number of newly infected individuals resulting from a single infected individual occurring in a totally healthy population has been referred to as the basic reproduction number (or ratio), $R_{0}$ (Diekmann et al., 1990). $R_{0}$ has been extensively used in human and animal epidemiology (e.g., Molisson, 1995). In botanical epidemiology, $R_{0}$ is the progeny-parent ratio, or 
the number of daughter lesions per mother lesion, when the mother lesion is established in a population of healthy individuals (Van den Bosch et al., 1988a; Zadoks and Schein, 1979; Van der Plank, 1963). The concept of $R_{0}$ has recently been the subject of strong interest in botanical epidemiology, under the mathematical framework of linked differential equations (e.g., Segarra et al., 2001; Madden et al., 2007).

The preliminary model in a broader context (3): linking $R_{c}$ and $R_{0}$ Considering the infectious - the reproductive - life-time, that is, from $t=0$ to $t=i$, of the first infection being established in a population of sites that are all susceptible, one may write:

$$
R_{0}=\int_{t=0}^{t=i} R c
$$

$R_{0}$ (also called gross reproduction) has also been shown to be an important parameter to consider when analyzing disease focus expansion (Van den Bosch et al., 1988a; 1988b). Links between developments in medical and botanical epidemiology in terms of $R_{0}$ and $R_{c}$ were discussed later on in several studies (e.g., Jeger and Van den Bosch, 1993). $R_{0}$ is a very appealing concept, because of its clear definition, its biological meaning, its possible decomposition in biological processes, and because it is an important factor determining epidemics. $R_{0}$ is, however, very difficult to estimate (Van den Bosch et al., 2008b). Several approaches have been offered, including:

- deriving equations relating $r$, the apparent rate of infection, or $r_{l}$, the logarithmic infection rate (sensu Van der Plank, 1963) to $R_{0}$ or $R_{c}$ (Van der Plank, 1963; Van den Bosch et al., 1988b; Sun and Zeng, 1994; Segarra et al., 2001);

- $\quad$ using matrix population models (Van den Bosch et al., 2008);

- experimental measurement (Van den Bosch et al., 1988); and

- $\quad$ approaches combining experiments and models (Allorent et al., 2005).

One way to see the preliminary epidemiological simulation model described here is that it allows the computation of the product of DMFR by $i$, at successive, discrete time steps. This product in turn corresponds to $R_{0}$. $R_{0}$ encompasses the entire infectious lifetime of a lesion, whereas $R_{c}$ considers each (infinitely small) time step over time during the infectious period. Thus, in the same way as $R_{c}, R_{0}$ varies over time and depends on weather variables, e.g., 
temperature and leaf wetness (e.g., Papastamati and Van den Bosch, 2007; Zadoks and Schein, 1979).

Van der Plank (1963) stated that no plant disease epidemic can start unless $R_{c} . i>1$, an inequality known to epidemiologists as 'the threshold theorem'. This inequality simply states that if an infectious site does not give rise to a new infection, then no epidemic would take place. Much work has elaborated on the threshold theorem (Madden et al., 2007).

\section{The mean field hypothesis}

Whichever approach is chosen, whether analytical or numerical, the model considered here is based on the major assumption that all healthy sites are equally accessible for infection, or that, conversely, propagules all have equal probabilities to reach and (possibly) infect healthy sites. This is what may be called a mean field hypothesis. Canopies are, in the real world, heterogeneous; leaves or fruits, or plant organs - sites, in general — are not all equally exposed to incoming inoculum; tissues vary in their susceptibility; gradients of propagule dispersal vary widely across pathosystems, and these gradients often depend on several, not one, dispersal mechanisms; or again, the microclimate in a canopy (say, in an apple tree, but in a wheat or rice field, too) is bound to show spatial variability. All these elements, which may explain why an epidemic occurs, or why it does not, are left aside at this stage. What has been shown in this chapter truly is a preliminary model. Simulation modeling is one approach that enables to explore further the 'what if' questions that we have.

\section{Simulations}

The STELLA® model provided with this chapter (EPIDEM.STMX) will allow you to explore the model structure and equations, and run the model with varying values of DMFR, $p, i$, and onset date of epidemics, to see the effects of such changes on the simulated epidemics. A listing of the program can be found in Appendix 4.1.

\section{Summary}

This chapter shows and assembles the building blocks of a preliminary epidemiological simulation model.

- The main equations governing the model are explained.

- Ways to initialize the model are shown. 
- The model behaves as expected, providing a verification of its structure and its equations.

- The model behaves as expected from pathosystems that correspond to its structure: variation in some key parameters (e.g., the durations of the latency and infectious periods, the intrinsic rate of disease increase - called here the daily multiplication factor, and the date of epidemic onset) translate in logical epidemiological patterns.

- Simulation modeling allows one to "see" the infection chain in action in a polycyclic process, with the compounding effects of parameter values influencing overlapping disease cycles of an epidemic.

- The many simplifying hypotheses of the model are discussed.

- The model structure developed in this chapter is discussed with respect to other current modeling approaches.

- A STELLA® model provided with this chapter (EPIDEM.STMX) can be used to see the effects of parameter changes on the simulated epidemics.

\section{References}

Allorent, D., Willocquet, L., Sartorato, A., and Savary, S. 2005. Quantifying and modelling the mobilisation of inoculum from diseased leaves and defoliated tissues in epidemics of angular leaf spot of bean. Eur. J. Plant Pathol. 113:377-394.

Brown, L. R. 2011. World on the Edge - How to Prevent Environmental and Economic Collapse. Earth Policy Institute. W.W. Norton \& Company, New York, London.

Cunniffe, N. J., Stutt, R. O. J. H., Van den Bosch, F., and Gilligan, C. A. 2012. Time-dependent infectivity and flexible latent and infectious periods in compartmental models of plant disease. Phytopathology 102:365-380.

Diekmann, O., Heesterbeek, J. A. P., and Metz, J. A. J. 1990. On the definition and computation of the basic reproduction ratio $\mathrm{R}_{0}$ in models for infectious diseases in heterogeneous populations. J. Math. Biol. 28:365-382.

Jeger, M. J., and Van den Bosch, F. 1993. Threshold criteria for model plant disease epidemics. I. Asymptotic results. Phytopathology 84:24-27.

Kranz, J., ed. 1974. Epidemics of plant diseases: Mathematical Analysis and Modeling. SpringerVerlag, Berlin.

Madden, L. V., Hughes, G., and Van den Bosch, F. 2007. The Study of Plant Disease Epidemics. American Phytopatholological Society, St. Paul, MN. 
Mollison, D., ed. 1995. Epidemic Models: their Structure and Relation to Data. Cambridge University Press, Cambridge, UK.

Papastamati, K., and Van den Bosch, F. 2007. The sensitivity of the epidemic growth rate to weather variables, with an application to yellow rust on wheat. Phytopathology 97:202-210.

Penning de Vries, F. W. T., and Van Laar, H. H., eds. 1982. Simulation of Plant Growth and Crop Production. Pudoc, Wageningen.

Savary S., Nelson A., Willocquet L., Pangga I., and Aunario J., 2012. Modelling and mapping potential epidemics of rice diseases globally. Crop Protection 34:6-17.

Segarra, J., Jeger, M. J., and Van den Bosch, F. 2001. Epidemic dynamics and patterns of plant diseases. Phytopathology 91:1001-1010.

Sun, P., and Zeng, S. 1994. On the measurement of the corrected basic infection rate. Zeitschrift für Pflanzenkrankheiten und Pflanzenschurtz 101:297-302.

Teng, P. S. 1983. Estimating and interpreting disease intensity and loss in commercial fields. Phytopathology 73:1587-1590.

Van den Bosch F, Zadoks, J. C., and Metz, J. A. J. 1988a. Focus expansion in plant disease. I. The constant rate of focus expansion. Phytopathology 78:54-58.

Van den Bosch F, Frinking, H. D., Metz, J. A. J., and Zadoks, J. C. 1988b. Focus expansion in plant disease. III: two experimental examples. Phytopathology 78:919-925.

Van den Bosch, F., McRoberts, N., Van den Berg, F., and Madden, L. V. 2008. The basic reproduction number of plant pathogens: Matrix approaches to complex dynamics. Phytopathology 98:239-249.

Van der Plank, J. E. 1963. Plant Diseases. Epidemics and Control. Academic Press, New York. de Wit, C. T., and Goudriaan, J. G. 1978. Simulation of Ecological Processes. Pudoc, Wageningen.

Zadoks, J. C. 1971. Systems analysis and the dynamics of epidemics. Phytopathology 61:600610.

Zadoks, J. C., and Schein, R. D. 1979. Epidemiology and Plant Disease Management. Oxford University Press, New York.

\section{Suggested reading}

Allen, T. H. F., and Starr, T. B. 1982. Hierarchy - Perspectives for Ecological Complexity. The University of Chicago Press, Chicago and London. 
Kranz, J. 1990. Epidemics, their mathematical analysis and modeling: an introduction. Pages 111 in: Epidemics of Plant Diseases. Second Edition. Kranz, J., Ed. Springer Verlag, Berlin.

\section{Exercises and questions}

Questions

1. A sub-process is
a. 1 hierarchy level below the process level
b. 2 hierarchy levels below the process level
c. 1 hierarchy level above the process level
d. 2 hierarchy levels above the process level

2. State variables of an epidemiological simulation model can include
a. the rate of infection
b. the duration of latency period
c. the number of latent sites
d. the correction factor

3. The rate of infection in an epidemiological simulation model can be written as
a. $\quad \mathrm{RG}=\operatorname{RemS} * \mathrm{DMFR}$
b. $\mathrm{RG}=(\mathrm{DMFR} * \mathrm{CORF} * \mathrm{InfS})+\mathrm{INOCPRIM}$
c. $\quad R G=($ DMFR $*$ INOCPRIM $)+($ CORF*InfS $)$
d. $R G=(C O R F * I n f S)+$ INOCPRIM

4. The dimension of the rate of infection is
a. $[\mathrm{N}]$
b. $\left[\mathrm{N} \cdot \mathrm{T}^{-1}\right]$
c. $\left[\mathrm{N} \cdot \mathrm{T}^{-2}\right]$
d. $\left[N \cdot N^{-1} \cdot T^{-1}\right]$

5. The following state variables may have an initial value set to zero 

a. number of healthy sites
b. number of latent sites
c. number of infectious sites
d. number of removed sites

Answers to questions

1. a: 1 hierarchy level below the process level.

2. c: the number of latent sites.

3. b: RG $=\left(\mathrm{DMFR}^{*} \mathrm{CORF}^{*} \operatorname{InfS}\right)+\mathrm{INOCPRIM}$

4. b: $\left[\mathrm{N} . \mathrm{T}^{-1}\right]$

5. b: number of latent sites, c: number of infectious sites, and d: number of removed sites.

Appendix 4.1. Program listing of EPIDEM

$\operatorname{HSites}(\mathrm{t})=\operatorname{HSites}(\mathrm{t}-\mathrm{dt})+(-\operatorname{INFECTION}) * \mathrm{dt}$

INIT HSites $=100000$

OUTFLOWS:

INFECTION $=($ DMFR $*$ CORF $*$ InfS $)+$ INOCPRIM

$\operatorname{InfS}(\mathrm{t})=\operatorname{InfS}(\mathrm{t}-\mathrm{dt})+($ TRANSFERT $-\operatorname{REMOVAL}) * \mathrm{dt}$

INIT InfS $=0,0,0,0,0,0,0,0,0,0$

TRANSIT TIME $=10$

INFLOW LIMIT $=$ INF

CAPACITY $=\mathrm{INF}$

INFLOWS:

TRANSFERT $=$ CONVEYOR OUTFLOW

OUTFLOWS:

REMOVAL = CONVEYOR OUTFLOW

LatS $(\mathrm{t})=\operatorname{LatS}(\mathrm{t}-\mathrm{dt})+($ INFECTION - TRANSFERT $) * d t$ 
INIT LatS $=0,0,0,0,0,0$

TRANSIT TIME $=6$

INFLOW LIMIT $=$ INF

CAPACITY $=$ INF

INFLOWS:

INFECTION $=($ DMFR $*$ CORF $*$ InfS $)+$ INOCPRIM

OUTFLOWS:

TRANSFERT $=$ CONVEYOR OUTFLOW

$\operatorname{RemS}(\mathrm{t})=\operatorname{RemS}(\mathrm{t}-\mathrm{dt})+(\operatorname{REMOVAL}) * \mathrm{dt}$

INIT RemS $=0$

INFLOWS:

REMOVAL $=$ CONVEYOR OUTFLOW

$\mathrm{ACI}=\mathrm{LatS}+\mathrm{InfS}+\mathrm{RemS}$

$\mathrm{CORF}=1-(\mathrm{ACI} /(\mathrm{ACI}+\mathrm{HSites}))$

DAY $=$ TIME

Dis $=\operatorname{InfS}+$ RemS

DMFR $=0.3$

INOCPRIM = IF $($ DAY=1) THEN 100 ELSE 0 


\section{Chapter 5. An Epidemiological Model Including Crop Growth and Senescence}

One of the many simplifying hypotheses that was made in developing the preliminary epidemiological model in the previous chapter concerns the number of sites, which was kept constant for the entire duration of epidemics. The implicit assumption was therefore made that a fixed initial stock of healthy sites was set at the beginning of an epidemic (and of a crop cycle), and left to decline under the effect of the rate of infection as an epidemic progresses. A crop that does not grow for 100 days does not exist. In this chapter, we want to account for crop growth, that is to say, the progressive build-up of healthy sites. Furthermore, we wish to account for the fact that, as time goes by, many sites senesce, implying that, as the crop grows and the epidemic builds up, fewer sites are made available to infection, not only because they might become infected (and so, not available anymore to infection), but also simply because they are senesced. Lastly, we would like to achieve these goals with as few, simple, hypotheses as possible.

\section{Adding components (and hypotheses) to the model}

Let us use the model developed in the previous chapter to incorporate crop growth and senescence. The overall structure of the modified model is shown in Fig. 5.1.

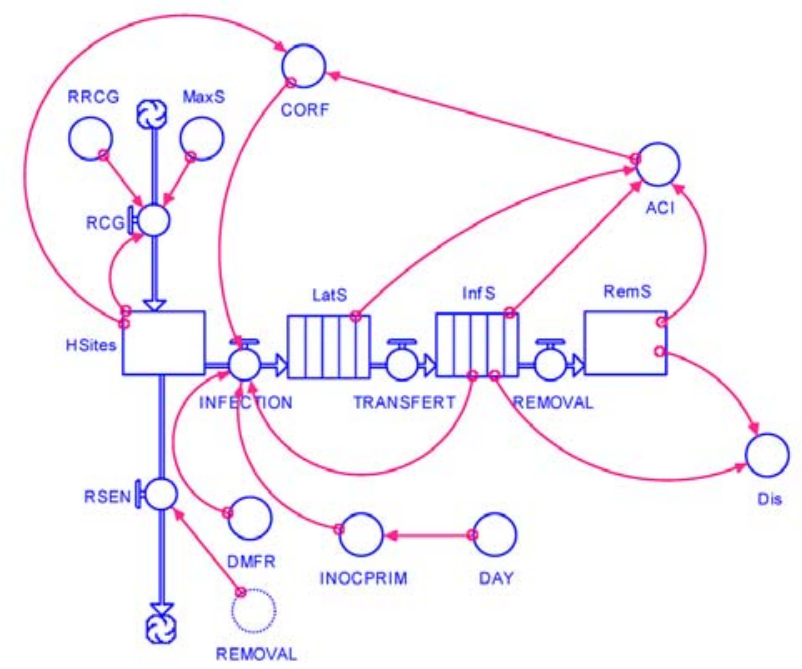

Figure 5.1. One needs to model crop growth, and thus create a rate of crop growth (RCG). For the sake of simplicity, let us assume that crop (site) growth is logistic. We thus have to create a carrying capacity, which represents the maximum size the site population may achieve, MaxS. 
One needs to model crop growth, and thus create a rate of crop growth (RCG). For the sake of simplicity, let us assume that crop (site) growth is logistic. We thus have to create a carrying capacity, which represents the maximum size the site population may achieve, MaxS.

As for all the site-variables (most of which are state variables in the model), the dimension of the parameter MaxS is [Nsite], although the considered system has a finite size (e.g., $1 \mathrm{~m}^{2}$ of a wheat crop in a large field). The proper dimension of site variables thus should be [Nsite. $\mathrm{L}^{-2}$ ]. However, from the onset of the previous chapter, the size of the system - again, an important hypothesis the model has - has been implicit. Thus, we shall retain [Nsite] as a dimension from now on.

The logistic rate of growth is proportional to the amounts of individuals (i.e., sites) that are contributing to growth. Let us further assume that only healthy sites (HSites) do so, that is to say that, once infected (latent, infectious, or removed), infected sites no longer contribute to growth. The rate of crop growth can therefore be written as:

\section{$\mathrm{RCG}=\mathrm{RRCG} *$ HSites $*(1-($ HSites/MaxS $))$}

Let us also assume that crop growth starts with a small number of sites, 100, with a carrying capacity of 100,000 sites ( $\mathrm{MaxS}=100,000)$, and a relative rate of growth of 0.1 site site ${ }^{1} \cdot$ day $^{-1}$. This latter assumption implies that each site gives birth to 0.1 new site at each time step. The result of crop (site) growth in the absence of disease is shown in Fig. 5.2.

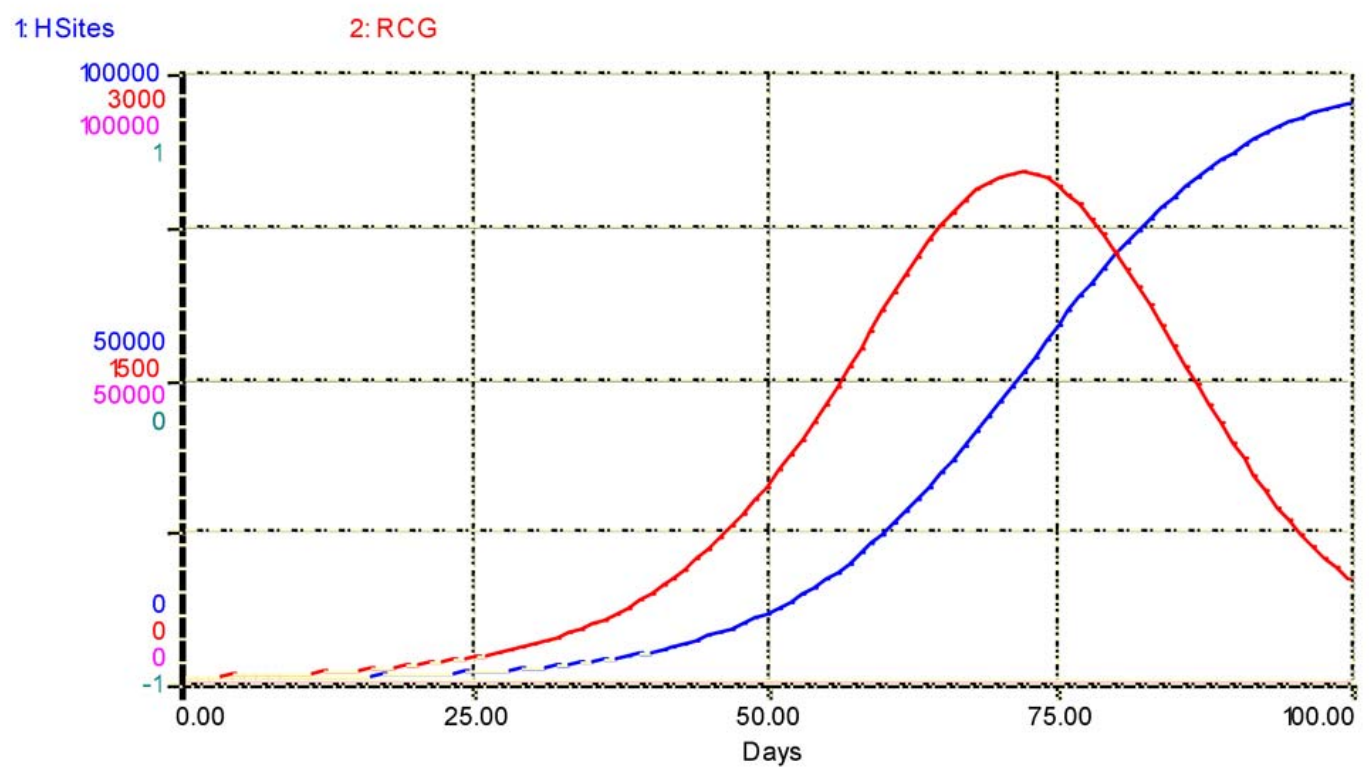

Figure 5.2. Simulated crop growth (number of sites, blue) and of crop growth rate (red) in a healthy crop. Horizontal axis: time (days); vertical axis: numbers of sites. 
We want next to incorporate senescence in the model. Senescence is indeed a very complex process (e.g., Gardener et al., 1985; Lim et al., 2003), which occurs in any crop stand, whether healthy or diseased. To incorporate such processes is tempting. Doing so would however imply involving complicated processes, and we would rather keep the model as simple and tractable as possible. A comparatively simple approach would at least involve including development, that is, the successive transition of sites through different physiological stages, in addition to growth, that is, in addition to the mere incrementing (and/or subtraction) of sites over time. Crop development will be addressed in the following chapters. What is proposed here is to take a shortcut, and assume that the rate of senescence (RSEN) is numerically equal to the rate of removal from the epidemiological process:

\section{RSEN = REMOVAL}

While another hypothesis will be discussed at the end of this chapter, let us ponder now what this equation implies. As the crop is being established, very little disease, if any (see below), is present. At that stage, one can safely assume that both removal and senescence are equal and null. In a second stage, once infected, sites go through the delays of latency and infectiousness. This takes a few days, corresponding to the latency period $p$ then the infectious period $i$. During this second stage, removal is at most small, and so is senescence. In a third stage, as the epidemic truly builds up, post-infectious sites start to accumulate as removed sites. In this third stage, senescence, too, tends to increase. Thus, for any disease, one may assume that equating the rates of senescence to that of removal is phenomenologically acceptable (they coincide over time), although this reasoning is not physiologically or epidemiologically correct; senescence occurs in absence of disease, in the same way as disease may not necessarily lead to senescence. If we take as an example a necrotrophic pathogen infecting the foliage, the assumption however appears appropriate; removed sites often senesce much faster. Then again, this is generally not the case in biotrophic pathogens, as with many leaf rusts on mono- or dicots. Let us accept this as a working hypothesis for the time being.

A third change has to do with the initiation of epidemics. In the preliminary model of the previous chapter, epidemics started off with an influx of 100 infections as soon as the process started. Since we now start off with 100 (healthy) sites, having them all infected at such an early stage of crop growth will not do; crop growth would stop immediately and such a thing is not realistic. Instead, the epidemic is started later on. 
In order to do so, we write INOCPRIM as:

IF (DAY=20) THEN 100 ELSE 0

instead of:

IF (DAY=1) THEN 100 ELSE 0

which was used in the model of Chapter 4. Furthermore, all the other parameters are set to the same default values as in Chapter 4.

Instead of changing the original structure of epidemic onset we now assume that 100 infections take place 20 days after crop growth has started. The listing of the program is given in Appendix 5.1 at the end of this chapter.

\section{Verifying the model: model behavior}

A first question is whether such changes have affected the model behavior: not only have crop growth and senescence been included, but the onset of disease has been delayed by 20 days. These are important changes that need to be addressed first.

Let us first compare the outputs of the model of Chapter 4 and those of the new model of Fig. 5.1, both of them simulating epidemics with onset times at $t=20$ days.

Fig. 5.3 shows dramatic differences in outputs. One is that, in the outputs of the new version of the model (Fig. 5.3b), we do not observe a steady decline of healthy sites over time. The new outputs show an initial increase then a decline. The maximum number of healthy sites is also strongly reduced. Other differences concern the diseased sites. Overall, the number of diseased sites is reduced when crop growth is taken into account. In the former (no crop growth, Fig 5.3a) simulation, one sees a sigmoid pattern followed by a decline of the latent sites. The new outputs show a regular increase of the infectious, removed, and accumulated diseased sites over time, while the progress curve for latent sites is sigmoid. These differences in behavior in the dynamics of the disease state variables are caused by the comparatively smaller amount of sites that are available (healthy) at the early stage of the epidemic, and which only increase progressively over time. Thus, at each time step over time, the amount of healthy sites, i.e. the carrying capacity of the disease to develop, varies: it initially increases at a moderate rate (because crop growth being logistic is proportional to a relatively small number of healthy sites), and then the carrying capacity tends to progressively decrease as disease intensifies. In simple words, one could say that the disease has less room to develop. 

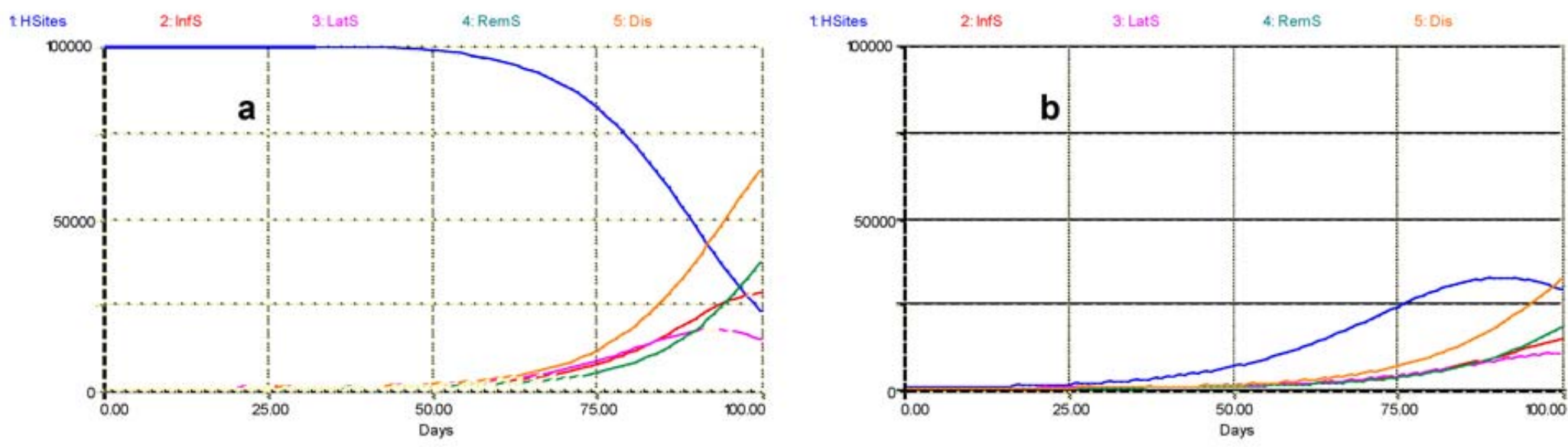

Figure 5.3. Simulated dynamics of sites (healthy, infectious, latent, removed, and total diseased) in a crop where growth and senescence are not (a, left) or are (b, right) simulated. Horizontal axis: time (days); vertical axis: numbers of sites

\section{Effect of varying rates of crop growth}

One important change in the initial model is therefore crop growth. We can conduct a sensitivity analysis, where the relative (or intrinsic) rate of growth is changed. Fig. 5.4 shows the outcomes, in terms only of healthy and diseased sites. With $\mathrm{RRCG}=0.05$, both crop growth and disease progress are negligible: this is because healthy sites are infected as soon as they are produced, preventing further growth (to which only healthy sites can contribute) occurring. With $\mathrm{RRCG}=$ 0.09 , some crop growth occurs, but diseased sites follow a regularly increasing curve, ending, in relative terms, with high disease intensity (i.e., Dis/(Dis+HSites), of roughly $70 \%$. With RRCG = 0.10 (which is the reference value in the earlier verifications), crop growth follows a sigmoid pattern, and diseased sites build up regularly, reaching, in relative terms, a fraction of disease lower than in the previous run. Thus at the end of the epidemic, intensity is about $50 \%$. Interestingly, this relative expression of terminal disease intensity is the same in the following runs. With $\mathrm{RRCG}=0.11$ or 0.15 , crop growth becomes faster, and shows a definite terminal decline. This decline is caused by the corresponding increase of disease, which now rapidly has a larger carrying capacity as the dynamics of growth unfolds. When $\mathrm{RRCG}=0.20$, crop growth reaches the maximum site carrying capacity (100,000 sites), but collapses as disease increases exponentially at a high rate: the accumulation of healthy sites provides 'room for maneuver' for disease increasing almost freely. 


\section{Effect of the daily multiplication factor, DMFR}

The previous chapter addressed a series of epidemiological parameters in their effects on the behavior of the model. Let us look at one of them, the daily multiplication factor (DMFR) of an infectious lesion. Three values for DMFR are tested in Fig. 5.5: DMFR $=0.30$, which is the standard value used in this chapter, and DMFR $=0.25$ or DMFR $=0.35$.
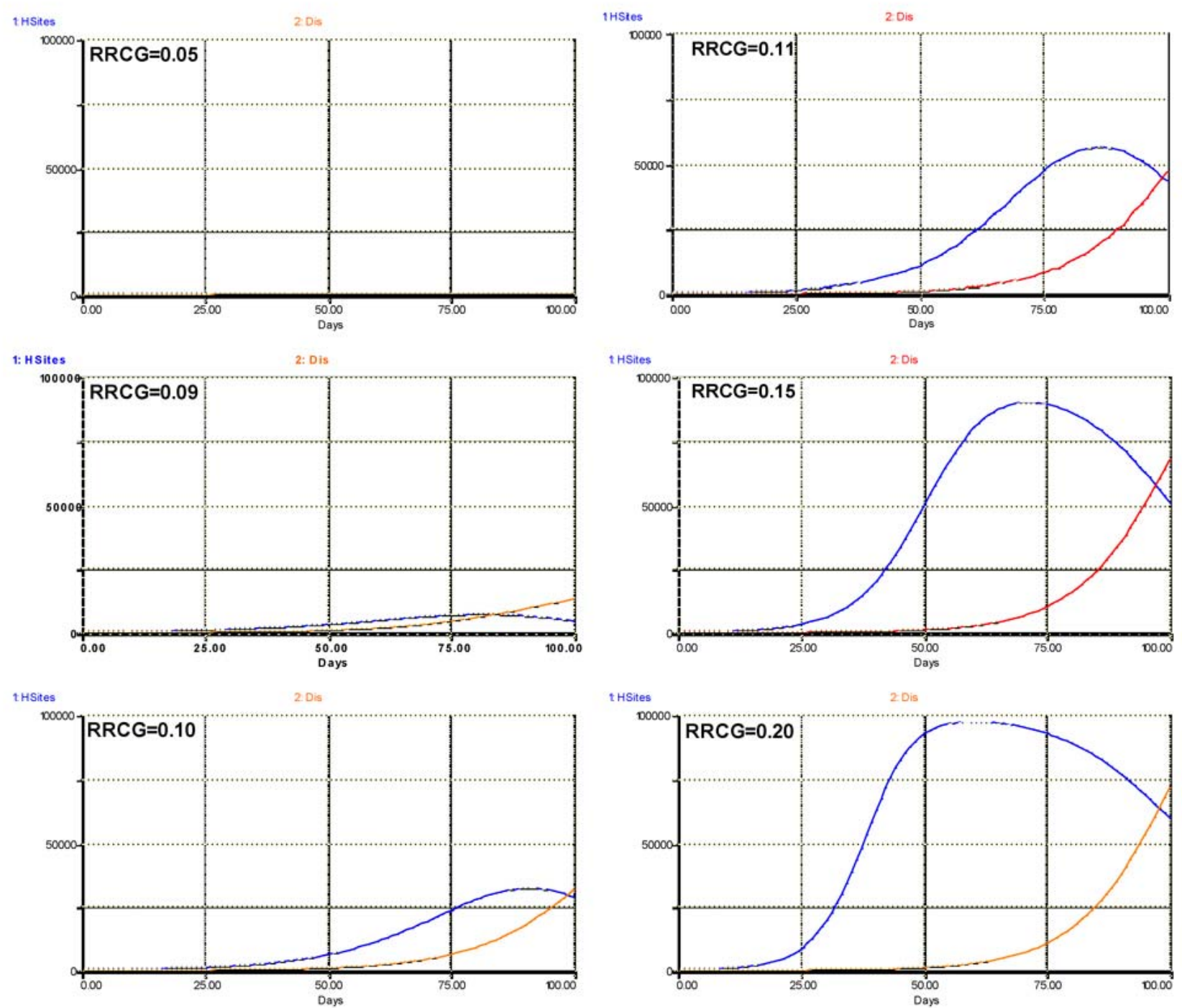

Figure 5.4. Simulated epidemics at varying relative rates of crop growth, RRCG. Graphs are showing only the simulated numbers of healthy and diseased sites. RRCG values are given in each graph. Horizontal axis: time (days); vertical axis: numbers of sites. 

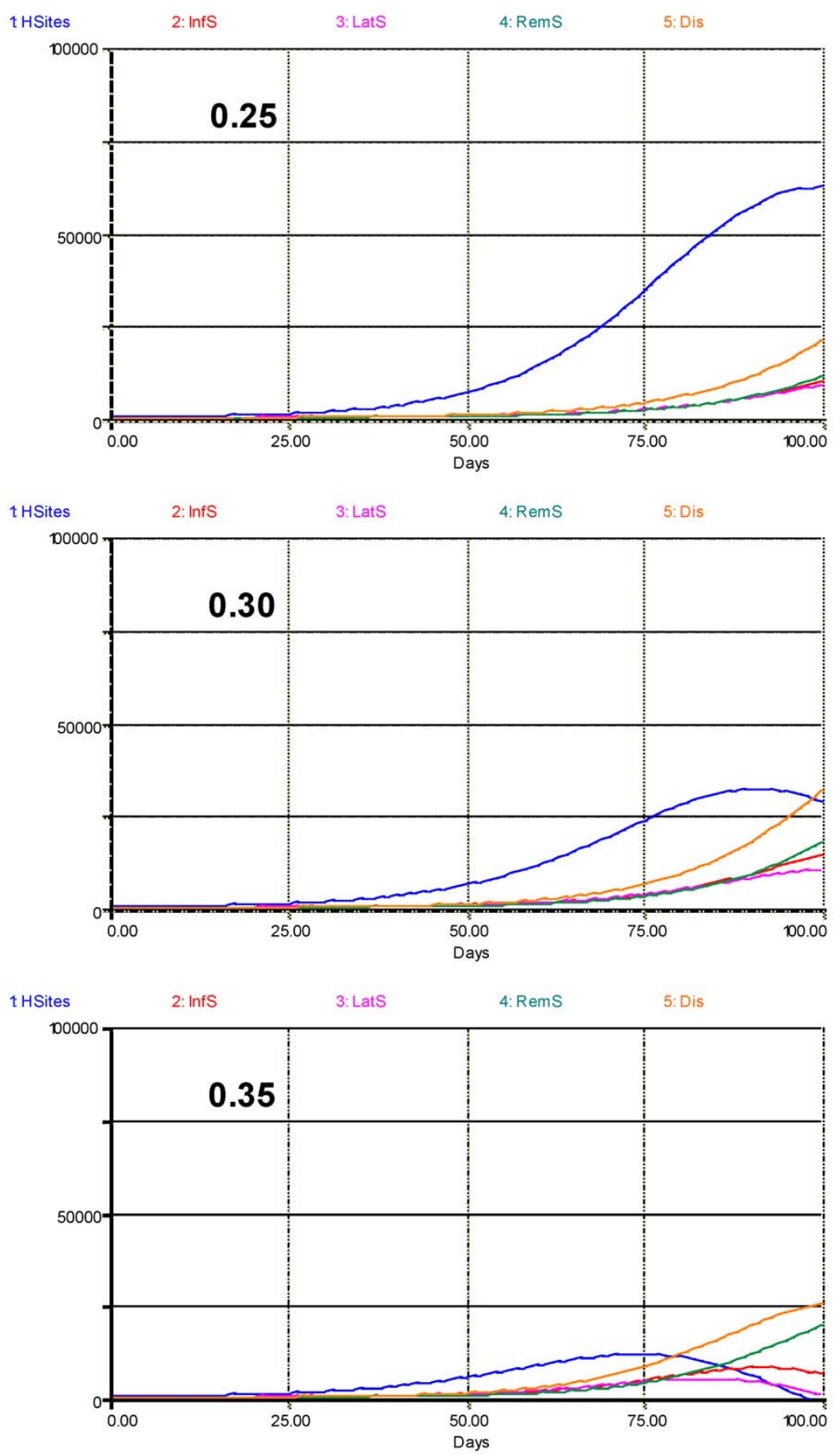

Figure 5.5. Simulated epidemics at varying values of DMFR: $0.25,0.30$, and 0.35 .

Graphs are showing the variation of healthy, latent, infectious, removed sites, and the total number of (visibly) diseased sites. 1: healthy sites; 2: infectious sites; 3: latent sites; 4: sites removed from the epidemiological process; 5: visibly diseased (infectious and removed) sites. Horizontal axis: time (days); vertical axis: numbers of sites. 
With DMFR $=0.25$, crop (site) growth follows a simple sigmoid pattern. Latent, infectious, and removed, as well as visibly diseased (infectious + removed) sites increase exponentially, hampering crop growth, but only to a marginal extent. By contrast, when DMFR is increased to a value of 0.35 , crop growth is strongly suppressed, and the number of healthy sites shows a clear decline at the end of the run. A similar decline also occurs for the latent and infectious sites, leading to a tapering-off of the visibly diseased sites.

These three runs also indicate that, with increased DMFR values, the terminal relative disease intensities, if expressed in absolute terms (i.e., [Nsite]), do not vary very much. However, if expressed in relative terms (i.e., \%, that is [Nsite.Nsite $\left.{ }^{-1}\right]$ ), a very strong decline is apparent. This additional set of runs thus underline the very strong difference in interpretation attached in expressing disease in absolute (that is, numbers, as the state variables used here) or in relative terms, which is so common in the epidemiological literature, where many results are reported as percentages.

\section{Modeling senescence in a different manner}

One of the hypotheses the model discussed so far implies that the rate of senescence is equal to the rate of removal of diseased sites. Even though explanations were given to support this hypothesis, at least from a phenomenological standpoint, further analysis is useful.

One could, for instance, assume that senescence of healthy sites is only related to plant physiology, and could be expressed by an intrinsic rate of ageing of healthy tissues or a rate of removal of healthy sites (RRemHS). One could also assume that senescence is influenced by both disease and ageing. One simple assumption in that case is to consider the two processes additive, that is, without interaction, and write:

\section{RSEN $=$ RRemHS*HSites + RRemDS*REMOVAL}

where RRemDS is an intrinsic rate of removal of healthy sites caused by disease.

The above equation was incorporated in the model and the following parameter values: RRemHS $=0.00001$ and RRemDS $=0.0001$ were used. These values are very small, because they are relative rates affecting processes that take place late in the growing season and the epidemic, and because the value for RRemDS, which actually is a modifier of a rate, is 10 times larger than the value of RRemHS (the rate of removal of diseased sites, Fig. 5.1). The outputs are shown in Fig. 5.6, and can be compared with the right hand-side of Fig. 5.3. Both graphs show much similarity, except for the decline of healthy sites at the end of the epidemic, which is more 
visible in Fig. 5.2. This is logical, since senescence is only caused by disease in this case. It thus seems that the earlier hypothesis made in the development of the model is acceptable.

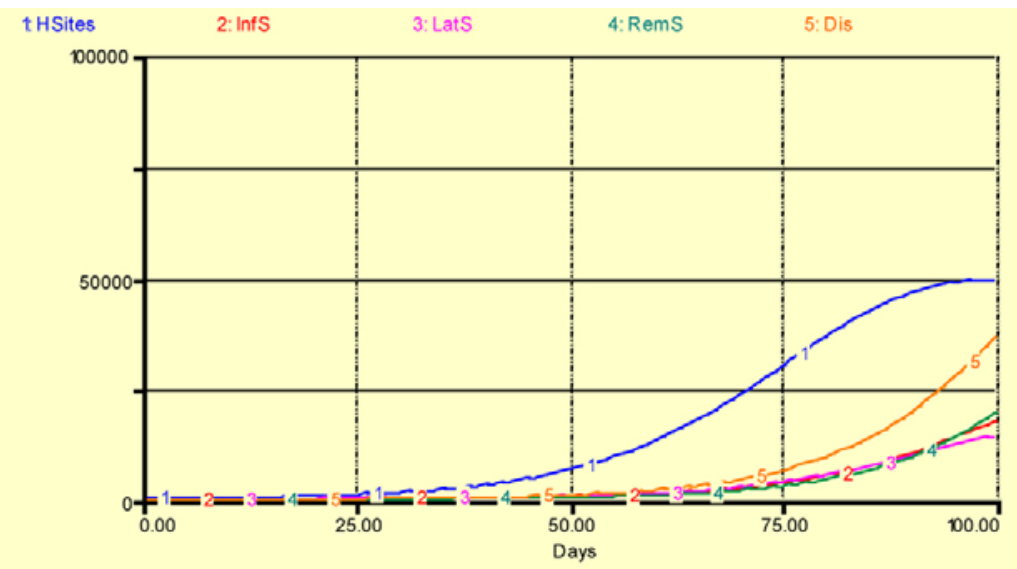

Figure 5.6. Simulated outputs with senescence of healthy tissues made dependent on both physiology and plant disease. 1: healthy sites; 2: infectious sites; 3: latent sites; 4: sites removed from the epidemiological process; 5: visibly diseased (infectious and removed) sites. Horizontal axis: time (days); vertical axis: numbers of sites.

\section{Revisiting hypotheses}

As in the previous chapter, the model used here implies a number of assumptions, some of which are explicit, and others, implicit. Let us address two explicit assumptions.

A first hypothesis is that crop (site) growth is logistic. In many cases, this assumption is not appropriate. This is especially the case if the sites under consideration were root-sites: root growth, in many crops, is very rapid in the beginning of the growing season, and stops long before the reproductive stage (in annual crops) is over (e.g., Gardner et al., 1985). The aerial tissues of a crop canopy also have an asymmetric rate of growth, which rapidly increases in the beginning of the growing season, and progressively decreases later. As a result, a flush of healthy sites would become rapidly available to possible infection, with strong differences in the shape of disease progress. There are a number of equations to account for such asymmetrical growth, for the host or for the pathogen (e.g., Kranz, J., 1976; 1990; Berger, 1981; Madden et al., 2007), and we leave it to the interested reader to try and incorporate them in the model, to see the epidemiological outcomes.

The second, actually very strong, hypothesis is that we assumed that only healthy sites (HSites) contribute to crop growth. This hypothesis can in turn be partitioned into two questions: 
(1) are all diseased sites truly not contributing to crop growth, and (2) are all healthy sites equally contributing to crop growth, creating a differential in disease occurring in some healthy sites rather than others.

The first question may, to some extent, be related to the biology of the considered pathogen. If one is dealing with a necrotroph (Cooke and Whipps, 1980), then infected sites become very rapidly dysfunctional (e.g., Savary et al., 1990; Bastiaans, 1993; Lopes and Berger, 2001), and the underlying hypothesis seems to hold. If one is dealing with a biotroph (e.g., Ayres, 1981; Mendgen, 1981), then infected sites still continue to be functional, but their photosynthetic activity is only directed to the maintenance, growth, and reproduction of the pathogen (e.g., Savary et al., 1990; Lopes and Berger, 2001). In the case of some host-pathogen interactions, the biotrophic pathogen in fact enhances the photosynthetic activity of infected tissues (e.g., Ayres, 1981). Thus, one may consider a possible under-estimate of the effect of infection on the population of sites, at least among the infected ones, and possibly, among the healthy sites. The latter remark brings us back again to the underlying hypothesis of evenly distributed disease in

plant tissues, in this case, in terms of crop growth - disease progress relationships. This topic will be further addressed in the next chapters. There are "shades of grey" in the trophic relationships between plants and their pathogens, since a great number of plant pathogens stand between the two extremes - biotroph vs. necrotroph.

The relationships between crop growth and epidemiological dynamics are, indeed, complex. The model structure presented here is a very simple one, to which many details would have to be added in order to address a specific pathosystem in any detail. The phrase "shades of grey" will return in the next chapter, in a completely different setting.

\section{Perspectives}

This chapter introduces in a very simple manner the complex linkages between a growing crop and the epidemic of a disease. Much has been written on the topic, which forms a strong component of disease management. The questions of relationships between the host and the pathogen, and between crop growth and disease (and harmful agents, in general) will be addressed further in the next chapters.

\section{Simulations}

The STELLA® model provided with this chapter (EPIDEMGRO.STMX) will allow you to explore the model structure and equations, and run the model with varying values of the 
intrinsic rate of crop growth, RRCG and DMFR, to see the effects of such changes on the simulated epidemics.

\section{Summary}

- Incorporating crop growth in a simulation model completely alters the course of an epidemic, whose shape becomes, overall, more realistic.

- Overall, crop growth provides room for maneuver for the course of epidemics, which depends on the dynamics of the available carrying capacity of the host population.

- The rate of crop growth itself has also a very strong effect on the modeled dynamics of epidemics and their outcomes.

- Many hypotheses might be implemented to model crop growth and senescence; in this section, these hypotheses were kept as simple as possible and the underlying assumptions are discussed.

- There can be very large implications in expressing disease intensity as an absolute amount of disease vs. as a fraction of host tissues, that is, disease percentage.

- A STELLA® model (EPIDEMGRO.STMX) allows you to explore the model behavior with varying values of the intrinsic rate of crop growth, RRCG and DMFR.

\section{References}

Ayres, P.G. 1981. Powdery mildew stimulates photosynthesis in uninfected leaves of pea plants. Phytopathologische Zeitschrift 88: 312-318.

Bastiaans L. 1993. Effects of leaf blast on growth and production of a rice crop. 1. Determining the mechanism of yield reduction. Netherlands Journal of Plant Pathology 99:323-334.

Berger, R.D. 1981. Comparison of the Gompertz and logistic equations to describe plant disease progress. Phytopathology 71:716-719.

Gardner, F.P., Pearce, R.B., and Mitchell, R.L. 1985. Physiology of Crop Plants. Iowa University Press, Ames.

Cooke, R.C., and Whipps, J.M. 1980. The evolution of modes of nutrition in fungi parasitic on terrestrial plants. Biological Reviews 55:341-360.

Kranz, J., 1976. Effect of some plant diseases on the loss of leaves. Zeitschrift fur Pflanzenkrankheiten und Pflanzenschutz 72:1373-1377. 
Kranz, J. (Ed.) 1990. Epidemics, their Mathematical Analysis and Modeling: an introduction. Pages 1-11 in: Epidemics of Plant Diseases. Second Edition, Springer Verlag, Berlin.

Lim, P. O., Woo H. R., and Nam H. G. 2003.Molecular genetics of leaf senescence in Arabidopsis. Trends in Plant Science 8:272-278.

Lopes, D.B., and Berger, R.D., 2001. The effects of rust and anthracnose on the photosynthesis competence of diseased bean leaves. Phytopathology 91:212-220.

Madden, L.V., Hughes, G., and Van den Bosch, F. 2007. The Study of Plant Disease Epidemics. The American Phytopathology Press. St Paul, Minnesota, USA.

Mendgen, K. 1981. Nutrient uptake in rust fungi. Phytopathology 71:963-964.

Robert, C., Bancal, M.O., and Lannou, C. 2002. Wheat leaf rust uredospore production and carbon and nitrogen export in relation to lesion size and density. Phytopathology 92:762-768.

Savary, S., De Jong, P.D., Rabbinge, R., and Zadoks, J.C. 1990. Dynamic simulation of groundnut rust, a preliminary model. Agricultural Systems 32:113-141.

\section{Suggested reading}

Berger, R.D. 1977. Application of epidemiological principles to achieve plant disease control. Annual Review of Phytopathology 15:165-183.

Jeger, M.J. 1986. Asymptotic behaviour and threshold criteria in model plant disease epidemics. Plant Pathology 35:355-361.

Jeger, M.J., and Van Den Bosch, F., 1994. Threshold criteria for model plant disease epidemics. I. Asymptotic results. Phytopathology 84:24-27.

Jeger, M.J. 2004. Analysis of disease progress as a basis for evaluating disease management practices. Annual Review of Phytopathology 42:61-82.

Zadoks, J.C., and Schein, R.D. 1979. Epidemiology and Plant Disease Management. Oxford University Press, New York.

Zadoks, J.C., and Rabbinge, R., 1985. Modelling to a purpose. Pages 231-244 In: Advances in Plant Pathology. Vol. 3. Mathematical Modelling of Crop Diseases. C.A. Gilligan, ed. Academic Press, London. 


\section{Exercises and questions}

Questions

1. The rate of crop growth with the hypothesis of carrying capacity can be written as
a. $\mathrm{RCG}=\mathrm{RRCG}^{*} \mathrm{HSites}$
b. $\mathrm{RCG}=\mathrm{RRCG} * \mathrm{MaxS} *(1-(\mathrm{HSites} / \mathrm{Max} \mathrm{S}))$
c. $\mathrm{RCG}=\mathrm{RRCG} * \mathrm{HSites} *(1+(\mathrm{HSites} / \mathrm{MaxS}))$
d. $\mathrm{RCG}=\mathrm{RRCG} *$ HSites $*(1-($ HSites/MaxS $))$

2. The dimension of the relative rate of crop growth, RRCG is
a. $[\mathrm{N}]$
b. $\left[\mathrm{N} \cdot \mathrm{T}^{-1}\right]$
c. $\left[\mathrm{N} \cdot \mathrm{N}^{-1} \cdot \mathrm{T}^{-1}\right]$
d. $\left[\mathrm{N} . \mathrm{T}^{-2}\right]$

3. An increase in the rate of crop growth will
a. increase the rate of infection
b. decrease the rate of infection
c. not affect the rate of infection

Answers to questions

1. d: RCG $=$ RRCG $*$ HSites $*(1-($ HSites/MaxS $))$

2. c: $\left[N \cdot N^{-1} \cdot T^{-1}\right]$

3. a: increase the rate of infection 
Appendix 5.1. Program listing of EPIDEMGRO

$\operatorname{HSites}(\mathrm{t})=\operatorname{HSites}(\mathrm{t}-\mathrm{dt})+(\mathrm{RCG}-\operatorname{INFECTION}-\mathrm{RSEN}) * \mathrm{dt}$

INIT HSites $=100$

INFLOWS:

RCG $=$ RRCG*HSites*(1-(HSites/MaxS))

OUTFLOWS:

INFECTION $=($ DMFR $*$ CORF $*$ InfS $)+$ INOCPRIM

RSEN $=$ REMOVAL

$\operatorname{InfS}(\mathrm{t})=\operatorname{InfS}(\mathrm{t}-\mathrm{dt})+($ TRANSFERT $-\operatorname{REMOVAL}) * d \mathrm{~d}$

INIT InfS $=0,0,0,0,0,0,0,0,0,0$

TRANSIT TIME $=10$

INFLOW LIMIT $=$ INF

CAPACITY $=$ INF

INFLOWS:

TRANSFERT $=$ CONVEYOR OUTFLOW

OUTFLOWS:

REMOVAL $=$ CONVEYOR OUTFLOW

LatS $(\mathrm{t})=\operatorname{LatS}(\mathrm{t}-\mathrm{dt})+($ INFECTION - TRANSFERT $) * d t$

INIT LatS $=0,0,0,0,0,0$

TRANSIT TIME $=6$

INFLOW LIMIT = INF

CAPACITY $=$ INF 
INFLOWS:

INFECTION $=($ DMFR $*$ CORF $*$ InfS $)+$ INOCPRIM

OUTFLOWS:

TRANSFERT $=$ CONVEYOR OUTFLOW

$\operatorname{RemS}(\mathrm{t})=\operatorname{RemS}(\mathrm{t}-\mathrm{dt})+(\operatorname{REMOVAL}) * \mathrm{dt}$

INIT RemS $=0$

INFLOWS:

REMOVAL $=$ CONVEYOR OUTFLOW

$\mathrm{ACI}=\mathrm{LatS}+\mathrm{InfS}+\mathrm{RemS}$

$\mathrm{CORF}=1-(\mathrm{ACI} /(\mathrm{ACI}+\mathrm{HSites}))$

$\mathrm{DAY}=\mathrm{TIME}$

Dis $=\operatorname{InfS}+$ RemS

$\mathrm{DMFR}=0.3$

INOCPRIM = IF (DAY=20) THEN 100 ELSE 0

$\operatorname{MaxS}=100000$

$\mathrm{RRCG}=0.1$ 


\section{Chapter 6. Modeling the Effects of Host Plant Resistance on Plant Disease Epidemics}

One of the many applications of simulation modeling in plant disease epidemiology pertains to host plant resistance, which can be seen as a major, and possibly the most important, contribution of botanical epidemiologists to sustainable agriculture (Johnson, 1984). The case of partial resistance deserves specific attention, because it illustrates very well the connection between experimental research and conceptual thinking encapsulated in modeling work. Studies on host plant resistance provide numerous examples of the research loop: induction - testing deduction. This section will show that simulation modeling can make this research loop forwardlooking, and enable research to consider the outcomes of choices. Recent advances in molecular plant pathology (Poland et al., 2009) actually now offer a bridge between epidemiology and molecular pathology through simulation modeling.

The induction phase involves (1) observing, exploring (experimentally), and designing the considered system and its structure (modeling); the testing phase (2) involves the measuring of epidemiological parameters, the (experimental) quantification of the system, that is, of epidemics, model parameterization, model verification, and comparing simulation outputs with field data (modeling); and the deduction phase involves (3) assessing levels of resistance of novel, existing or potential, genetic material. Modeling can thus become a very powerful tool for phenotyping host plant resistance.

\section{The nature of host plant resistances}

Host plant resistance in plants comes in many forms. Early works (Van der Plank, 1963; Flor, 1946; 1971; Agrios, 2005), from the "host point of view", emphasized horizontal or vertical resistances, and from the "pathogen point of view", emphasized specificity or non-specificity. After decades of research, these clear, almost Manichean, divides have come to be questioned. From the host point of view, the very nature of host plant resistance is now seen as a much more complex phenomenon than initially thought, with many different facets and outcomes. Complete, pathogen-specific, resistance, which was long perceived fragile, because it can, on principle, easily be overcome by pathogen populations under heavy selection pressure, is now seen from a different perspective; some of these complete resistance genes do appear to confer durable resistance (Poland et al., 2009). In other words, there are different types of complete resistance. These different types of complete resistance are thus mirrored by differences in the genetic make- 
up of pathogen populations. Indeed, durable host plant resistance has been, and still is, the ultimate goal of plant pathologists, geneticists, and breeders alike (e.g., Robinson, 1976; Bonman et al, 1992). The reasons for the central importance of this goal is that durable resistance, a science-based, seed-borne technology, can comparatively be easily deployed, and does not have negative environmental impacts on human and animal health. Durable resistance can also be readily available, especially if carried by inbred (or perennial) varieties, to resource poor farmers, who still represent the bulk of the world farmers' population today.

One often speaks today of qualitative resistance (QLR) and quantitative resistance (QDR). Quantitative (i.e., partial, or incomplete) resistance comes in different shades of grey (Poland et al, 2009). It has been recognized long ago that qualitative resistance may be associated to one locus, and that it can be overcome (e.g., Eversmeyer and Kramer, 2000). This has for instance led to the recent massive epidemic of new strains of wheat stem rust (caused by Puccinia graminis Ug99) that are virulent on cultivars carrying widely deployed R-genes (Stokstad, 2007). More recently, QLR genes have been shown to vary, some of them providing long-lasting resistance despite the selection pressure they cause (Poland et al., 2009). Recent results suggest that QDR and QLR may in part be actually determined by the same genetic bases, which had long been envisioned (e.g., Parlevliet and Kuiper, 1977). The latter point might perhaps explain why some QLR provide more durable resistance - being associated with QDR.

Simulation modeling offers a critical tool to bridge knowledge and understanding between molecular geneticists, breeders, and plant disease epidemiologists. One main reason is that simulation modeling enables one to 'see' what otherwise could not be monitored at the systems level - be it plant, field, or region. Another reason is that simulation modeling can provide a very strong tool to help phenotyping genetic materials (e.g., Zadoks, 1977; Rapilly, 1979; Savary et al. 1990; Andrade-Piedra et al., 2005), which perhaps represents the main bottleneck of breeding programs today.

\section{Components of resistance: general definitions and operational definitions}

There is a great deal of difference between a general definition and an operational definition (Zadoks, 1972a), which however are sometimes confused. General definitions can be phrased through sentences and refer to concepts. General definitions are open to debate and offer the possibility of sharing among a large number of scientists. Operational definitions, on the other hand, are developed under the premise that the general definition is accepted and are phrased in a 
practical, often numerical or algebraic form. Operational definitions are thus the practical implementation of the general definitions they correspond to, and thus can be seen as 'recipes', to apply concepts in a specific context. Operational definitions may enter in such detail that they lose the general, conceptual, value of the general definitions they were borne from. The case of components of resistance is one good example of the translation of general definitions to operational ones.

A component of partial resistance (i.e., of quantitative resistance), as a general definition, is one independent element of a chain that contributes to hampering, to some degree, disease progress. If a combination of components of partial resistance affects the disease cycle collectively, epidemics may be suppressed. Considering the classical infection chain (Kranz, 1990), which connects each individual stage of a pathogen's life cycle (which could be seen as a state variable of the disease cycle seen as a system of its own), one should further consider that components of resistance must not overlap, because each of them affect one specific stage of the disease cycle (Zadoks, 1972b).

The latter remark may have important practical applications. In times where phenotyping host plant resistance has become the most difficult part of breeding programs, it may be critical to be able to link a given QTL or gene to a particular component of resistance. Phenotyping for host plant resistance, especially for partial resistance, which has been elusive for so many decades, and might be within reach given the molecular tools available today, could thus remove a bottleneck of many breeding programs, at least for quite a few pathosystems.

\section{Arithmetic operational definitions of components of resistance}

Operational definitions for components of partial resistance corresponding to the epidemiological model discussed in the previous chapters have been developed by Zadoks and Parlevliet in a series of publications (Zadoks, 1972b; Parlevliet, 1977; 1979; Parlevliet and Zadoks, 1977). A component of partial resistance is a dimensionless relative resistance coefficient, RR, which varies between 0 and 1 :

$$
0 \leq \mathrm{RR} \leq 1
$$

in which 1 corresponds to the highest level of resistance for this component (which means that no further progress in the disease cycle is made beyond the corresponding stage of the cycle), while 0 corresponds to maximum susceptibility. In other words, when $R R=1$, the disease cycle is 
stopped at the corresponding stage of the disease cycle, and the epidemic halts; if $R R=0$, there is full susceptibility, and the pathogen is allowed to pass this stage unhampered.

In the prototype epidemiological model developed so far, we can consider four components of resistance: for infection efficiency (IE): RRIE; for sporulation (SP): RRSP; for latency period duration (LP): RRLP; and for infectious period duration (IP): RRIP.

The previous chapters have shown that while resistance increases with smaller IE, SP, and shorter IP, resistance decreases with shorter LP. Thus, the equations for relative resistances will vary depending on the direction with which decreasing observed values of IE, SP, LP, and IP will be. Operational definitions are meant to use observations, and what is observed by breeders is always relative. Large breeding programs always have a reference. When it comes to partial resistance, one can never be sure to have, within a given field experiment, the highest possible level of resistance. But what is available is the currently lowest level of resistance, a reference for susceptibility, which can be used as a control. In the case of partial resistance, the best practical reference therefore is a susceptible cultivar, $\mathrm{c}$.

Assuming that the cultivar to be tested is denoted $\mathrm{x}$ and that the control is denoted $\mathrm{c}$, the operational definitions for components of partial resistances therefore can be written as relative resistance terms $(\mathrm{RR})$, which are functions of $\mathrm{x}$ and $\mathrm{c}$ :

$$
\begin{aligned}
& \operatorname{RRIE}(x)=1-[\operatorname{IE}(x) / \operatorname{IE}(c)] ; \\
& \operatorname{RRSP}(x)=1-[\operatorname{SP}(x) / \operatorname{SP}(c)] ; \text { and } \\
& \operatorname{RRIP}(x)=1-[\operatorname{IP}(x) / \operatorname{IP}(c)] .
\end{aligned}
$$

These equations are based on the assumption that a cultivar $\mathrm{x}$ will have values of IE, SP, and IP smaller than (or equal to) the susceptible control. Note that, because $\operatorname{IE}(\mathrm{x}) \leq \operatorname{IE}(\mathrm{c}), \operatorname{SP}(\mathrm{x}) \leq$ $\mathrm{SP}(\mathrm{c})$, and $\operatorname{IP}(\mathrm{x}) \leq \mathrm{IP}(\mathrm{c})$, all these terms follow the above condition: $0 \leq \mathrm{RR} \leq 1$. This is because, at the highest possible level of observed susceptibility: $\operatorname{IE}(x)=\operatorname{IE}(c), \operatorname{SP}(x)=\operatorname{SP}(c)$, and $\operatorname{IP}(x)=$ $\operatorname{IP}(\mathrm{c})$.

In the case of latency period duration, however, resistance will correspond to higher LP values. Thus a slightly different equation:

$$
\operatorname{RRLP}(x)=1-[\operatorname{LP}(c) / \operatorname{LP}(x)] \text {. }
$$

As indicated earlier, RRIE, RRSP, RRIP, and RRLP are relative resistance terms, each of which corresponding to a unique, non-overlapping, step of the infection chain: therefore, they can be called components of resistance. 
The question then arises on how to combine these components, that is, how to express the relative resistance of a host genotype that carries different levels of each of the separate, independent, components of resistance. The combined relative resistance, $\mathrm{RR}_{\mathrm{c}}$, of a variety carrying several components of resistance must meet the following conditions (Zadoks, 1972b; Savary et al., 1990):

a) $0 \leq R_{c} \leq 1$;

b) if all the relative resistances (RRi) corresponding to all components (i) are null, then the combined relative resistance $\left(\mathrm{RR}_{\mathrm{c}}\right)$ is null;

c) if any one of the values of the $\mathrm{p}$ components is equal to 1 , then $\mathrm{RR}_{\mathrm{c}}=1$;

d) if any one of the values of the components is $\neq 0$, then $R_{c} \neq 0$.

Such a set of conditions are met by the following equation (Savary et al., 1988):

$$
\begin{gathered}
\mathrm{p} \\
\operatorname{RR}_{\mathrm{c}}=1-\{\Pi(1-\mathrm{RRi})\}
\end{gathered}
$$

where $\mathrm{p}$ is the number of components of resistance involved.

For instance:

if:

$$
\begin{aligned}
& \mathrm{RRIE}=\mathrm{RRSP}=\mathrm{RRIP}=\mathrm{RRLP}=1, \text { then } \\
& \begin{aligned}
\mathrm{RR}_{\mathrm{c}} & =1-\{(1-\mathrm{RRIE}) *(1-\mathrm{RRSP}) *(1-\mathrm{RRIP}) *(1-\mathrm{RRLP})\} \\
& =1-\{(1-1) *(1-1) *(1-1) *(1-1)\}=1-0
\end{aligned}
\end{aligned}
$$

and: $\mathrm{RR}_{\mathrm{c}}=1$

if:

RRIE $=$ RRSP $=$ RRIP $=$ RRLP $=0$, then

$\mathrm{RR}_{\mathrm{c}}=1-\{(1-\mathrm{RRIE}) *(1-\mathrm{RRSP}) *(1-\mathrm{RRIP}) *(1-\mathrm{RRLP})\}=1-1$

and: $\mathrm{RR}_{\mathrm{c}}=0$

if:

$\mathrm{RRIE}=\mathrm{RRSP}=\mathrm{RRIP}=\mathrm{RRLP}=0.5$, then

$\mathrm{RR}_{\mathrm{c}}=1-\{(1-0.5) *(1-0.5) *(1-0.5) *(1-0.5)\}=1-\left\{1-0.5^{4}\right\}=$

and: $\mathrm{RR}_{\mathrm{c}}=0.9375$

if one of the components of resistance takes a value of 1 (the disease cycle cannot proceed), for example the first component: $\mathrm{RRIE}=1$, while $\mathrm{RRSP}=\mathrm{RRIP}=\mathrm{RRLP}=0$, then $\operatorname{RR}_{\mathrm{c}}=1-\{(1-1) *(1-0) *(1-0) *(1-0)\}=1-\{1-(0 * 1 * 1 * 1)\}=1-1$ and: $\mathrm{RR}_{\mathrm{c}}=0$ 
The above equation for calculating $\mathrm{RR}_{\mathrm{c}}$ on the basis of individual components of resistance is well known to plant pathology. Long ago, G.W. Padwick (1956) used the same equation to assess crop losses in the British colonies, the idea being that what has been lost once cannot be lost twice, thus a product of successive terms.

But this equation can be interpreted in a third way. From a probabilistic stand point, calculating $\mathrm{RR}_{\mathrm{c}}$ as a product implies that the values taken by each different (non-overlapping) component of resistance reflect independent events. Calculating $\mathrm{RR}_{\mathrm{c}}$ with the above formula thus assumes that components of resistance, as traits, are governed by independent genetic bases, thus assuming a bridge between phenotypic expression and genetic information. If such were the case, one could envision a straightforward relationship between the phenotypic make-up of a given variety (its field response) and its genetic make-up. Unfortunately components of resistance, however, are often correlated in their phenotypic expression (e.g., Savary et al., 1988) and may have common genetic bases (Poland et al, 2009).

\section{Operational definitions of components of resistance in simulation modeling}

The earlier arithmetic equations for components of resistance can be translated for simulation modeling purposes. For a given cultivar $\mathrm{x}$, the epidemiological parameters IE, LP, SP, and IP, can be corrected according to the corresponding components of resistance as follows:

$$
\begin{aligned}
& \mathrm{IEcor}=\mathrm{IE}(\mathrm{x})=\mathrm{IE}(\mathrm{c}) *(1-\mathrm{RRIE}) ; \\
& \mathrm{SPcor}=\mathrm{SP}(\mathrm{x})=\mathrm{SP}(\mathrm{c}) *(1-\mathrm{RRSP}) ; \\
& \mathrm{IPcor}=\mathrm{IP}(\mathrm{x})=\mathrm{IP}(\mathrm{c}) *(1-\mathrm{RRIP}) ; \text { and } \\
& \mathrm{LPcor}=\mathrm{LP}(\mathrm{x})=\mathrm{LP}(\mathrm{c}) /(1-\mathrm{RRLP})
\end{aligned}
$$

Simulation modeling allows addressing each of the processes of the disease cycle independently and the components of resistance that are attached to them. Over the course of an epidemic, disease cycles may quickly overlap and the effect of a given component of resistance can no longer be detected by the observer. A main advantage of simulation modeling compared to the arithmetic approach outlined above is that it readily enables analyzing the effects of components of resistance on epidemics. In particular, modeling enables disentangling the individual effects of components of resistance in an explicit manner. 


\section{Implementing components of resistance in a simulation model}

The four components of resistance described above can easily be incorporated in the simplified model developed so far. In a first stage RRIE and RRSP will be addressed, then RRLP, and lastly RRIP.

In the previous chapters, the rate of infection was calculated as:

INFECTION $=($ DMFR $*$ CORF $*$ InfS $)+$ INOCPRIM, where INFECTION is the rate of new infections per day (dimension: $\left[\mathrm{N}^{-T^{-1}}\right]$ ), DMFR is our numerical equivalent of Van der Plank's (1963) rate of infection corrected for removals (i.e., the number of new infections per lesion per day $\left.\left[\mathrm{N} \cdot \mathrm{N}^{-1} \cdot \mathrm{T}^{-1}\right]\right), \mathrm{CORF}$ is the fraction of sites still available to infection ([N. $\left.\left.\mathrm{N}^{-1}\right]\right), \operatorname{InfS}$ is the current number of infectious sites $([\mathrm{N}])$, and INOCPRIM is an inflow of infectious propagules ([N.T $\left.\left.{ }^{-1}\right]\right)$.

Let us consider DMFR in a little more detail, and say that it consists of the product of two components: first, the inflow of propagules (spores) that may lead to infections, and second, the infection efficiency of these propagules (Zadoks, 1971). Thus:

$\mathrm{DMFR}=\mathrm{IE} * \mathrm{SP}$, where:

$\mathrm{DMFR} \equiv\left[\mathrm{N}_{\text {lesion }} \cdot \mathrm{N}_{\text {lesion }}{ }^{-1} \cdot \mathrm{T}^{-1}\right]$;

Botanical epidemiology deals with two interacting populations: that of the plant host, which is represented by sites, and that of pathogen units. Pathogen units may take many forms, but for the sake of simplicity, let us assume that two types of pathogen units are considered here: lesions and propagules. The translations of lesions into propagules, and from propagules into lesions, are complex processes. We cannot address here the extremely diverse range of life cycle strategies plant pathogens have, despite their importance. What is important at this stage is to be are aware of the possibility of these transitions: some lesions produce propagules, and some propagules (sometimes, very few indeed) produce lesions. Thus, speaking of the number of pathogen units, one may use the same dimension: $\left[\mathrm{N}_{\text {lesion }}\right] \equiv\left[\mathrm{N}_{\text {propagule }}\right]$.

IE is the number of lesions generated per effective propagule (i.e., effectively coming into contact with the host):

$$
\mathrm{IE} \equiv\left[\mathrm{N}_{\text {lesion }} \cdot \mathrm{N}_{\text {propagule }}^{-1}\right] \equiv\left[\mathrm{N}_{\text {lesion }} \cdot \mathrm{N}_{\text {lesion }}^{-1}\right] \text {; }
$$

and SP is the number of propagules produced per lesion per unit time:

$$
\mathrm{SP} \equiv\left[\mathrm{N}_{\text {propagule }} \cdot \mathrm{N}_{\text {lesion }}{ }^{-1} \cdot \mathrm{T}^{-1}\right] \equiv\left[\mathrm{N}_{\text {lesion }} \cdot \mathrm{N}_{\text {lesion }}{ }^{-1} \cdot \mathrm{T}^{-1}\right] \text {. }
$$


SP is thus directly linked to InfS, the number of infectious sites, and their ability to produce propagules that may cause new infections, and IE accounts for the efficiency of these propagules to infect. The equation for INFECTION can then be written as:

$$
\mathrm{INFECTION}=(\mathrm{IEcor} * \mathrm{SPcor}) * \operatorname{InfS} * \mathrm{CORF}+\mathrm{INOCPRIM}
$$

with

$$
\mathrm{IEcor}=\mathrm{IE}(\mathrm{c}) *(1-\mathrm{RRIE})=0.3 *(1-\mathrm{RRIE}),
$$

since IE(c) $=0.3$ is the infection efficiency of the reference susceptible cultivar, and

$$
\mathrm{SPcor}=\mathrm{SP}(\mathrm{c}) *(1-\mathrm{RRSP})=1 *(1-\mathrm{RRSP}),
$$

since $\mathrm{SP}(\mathrm{c})=1$ is the term used for the reference susceptible cultivar (i.e., each lesion produces one propagule that potentially may lead to infection at each time step); this also amounts to the equivalency: $\mathrm{SP}=\mathrm{InfS}$.

RRLP and RRIP, being delay functions, are handled differently in the model. In the case of the latency period duration, longer LP values correspond to stronger resistance, while it is the opposite in the case of the infectious period. Thus:

$$
\text { LPcor }=\mathrm{LP}(\mathrm{c}) /(1-\mathrm{RRLP})=6 /(1-\mathrm{RRLP})
$$

since 6 days was the default value for the residence time of lesions in the latent stage. Since this is a residence time, this change is not made on the inflow to the state variable for latent lesions, LatS (which would amount to changing the rate of infection) but to the rate of outflow from the latent stage. Similarly:

$$
\mathrm{IPcor}=\mathrm{IP}(\mathrm{c}) *(1-\mathrm{RRIP})=10 *(1-\mathrm{RRIP})
$$

since 10 days was the value chosen to represent maximum susceptibility in the previous versions of the model. Again, this change is applied to the outflow from the infectious stage, towards removal.

These changes are shown in the flow diagram of Fig. 6.1, and the program listing is provided in Appendix 6.1. 


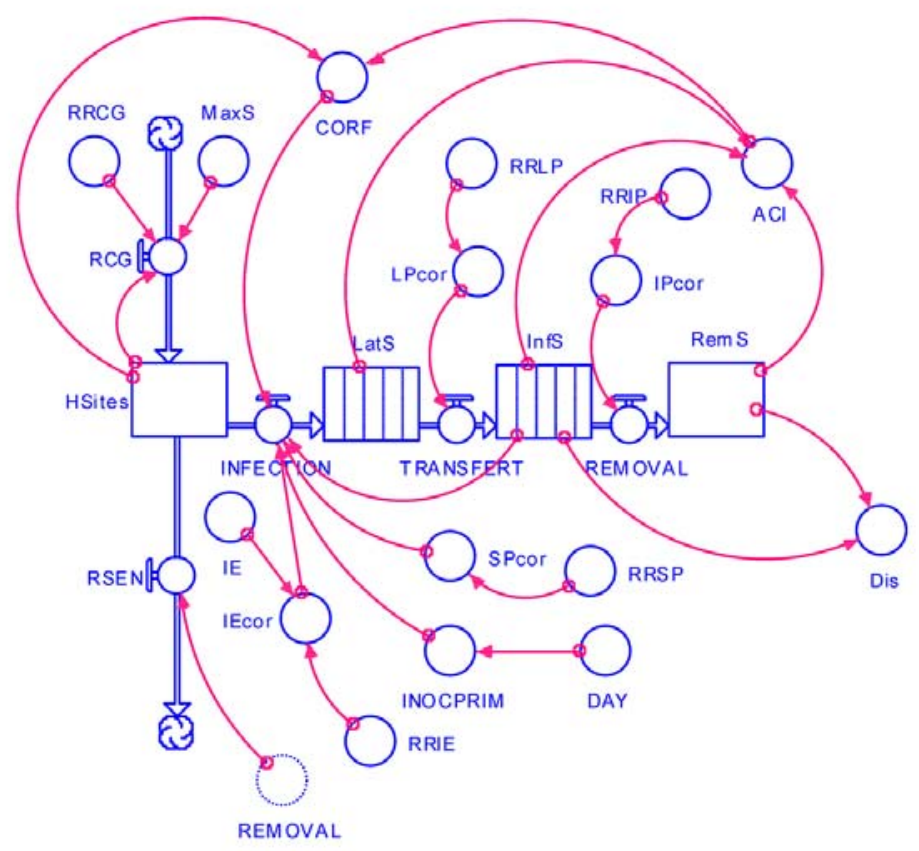

Figure 6.1. Flowchart of an epidemiological simulation model incorporating components of resistance.

\section{Important remarks}

In the above paragraph, we considered DMFR as the product of the inflow of propagules (spores) that may lead to infections by the infection efficiency of (effective) propagules, i.e.: $\mathrm{DMFR}=\mathrm{IE} * \mathrm{SP}$. This enabled us to incorporate in the model components of resistance for infection efficiency and propagule production.

What is meant here by "propagule production" needs clarification. In the case of an aerially-dispersed pathogen, the classical stages are: "propagule formation", "liberation", "transport", and "deposition" (e.g., Kranz, 1978; Zadoks and Schein, 1979). Equivalents could be determined (and operationally defined; Butt and Royle, 1980) in the case of vector-borne (e.g., Madden et al., 2000) or soil-borne (e.g., Gilligan, 1990) diseases.

Therefore, what we refer to here as "propagule formation" (SP) covers, in an admittedly loose way, several, complex, and concatenated processes of the infection chain. Specifically, for a vector-borne pathogen, SP refers to the number of propagules that have been acquired from a mother lesion, then transmitted and inoculated to a target host site, which may become a daughter lesion. 
The very notion of lesion may vary from disease to disease. In some cases, individual root segments or fractions of leaves will do; in others, one will better focus on shoots or fruits; and in many diseases, one should consider entire plants. For instance, in the case of many vector borne diseases, the notion of lesion may be a whole plant or a tree (Savary et al. 2012).

Again, this brings us back to the critical issue of choosing the right state variables of a system and its limits, which were introduced in Chapter 1.

Let us focus a little more on aerially dispersed diseases. In this case, SP refers to those propagules that have been produced, liberated, transported, and deposited on a host site (whether infected or not). In the case of many aerially dispersed pathogens, the corresponding lesion often is a fragment of host tissue (e.g., a spot on leaf or fruit) or a fragment of host unit (e.g., a shoot, or a branch; Savary et al. 2012). Thus, SP is a shortcut, and refers to the number of propagules that are effectively made available for potential infection, per unit time and per "mother" lesion.

Still, much detail could be experimentally measured and accounted for in a mechanistic model. In the case of peanut rust, for instance (Savary et al., 1990), the above processes, and others, were taken into account through relative rates of:

- liberation (which depends on relative humidity and the occurrence of rainfall),

- exhaustion of uredosori after a dispersal event (which depends on relative humidity and the occurrence of rainfall),

- deposition (which depends on whether the canopy is dry or not),

- survival and death of spores after they have been deposited (a function of time since liberation),

- $\quad$ spore leaching from pustules in the canopy if rainfall exceeds a given threshold.

Whether such details truly are necessary must be pondered. This was not incorporated in this modeling exercise.

\section{Simulated effects of components of resistance}

A first question one may want to address is whether and to what extent the combinations of components of resistance may suppress epidemics. In a first set of runs all the components of resistance were made equal ( $R R i)$, and three runs were performed: $R R i=0$ (no partial resistance), $\mathrm{RRi}=0.1$ and $\mathrm{RRi}=0.2$. The results are shown in Fig. 6.2, with very strong effects, even when all components of resistance are set to 0.1 . 

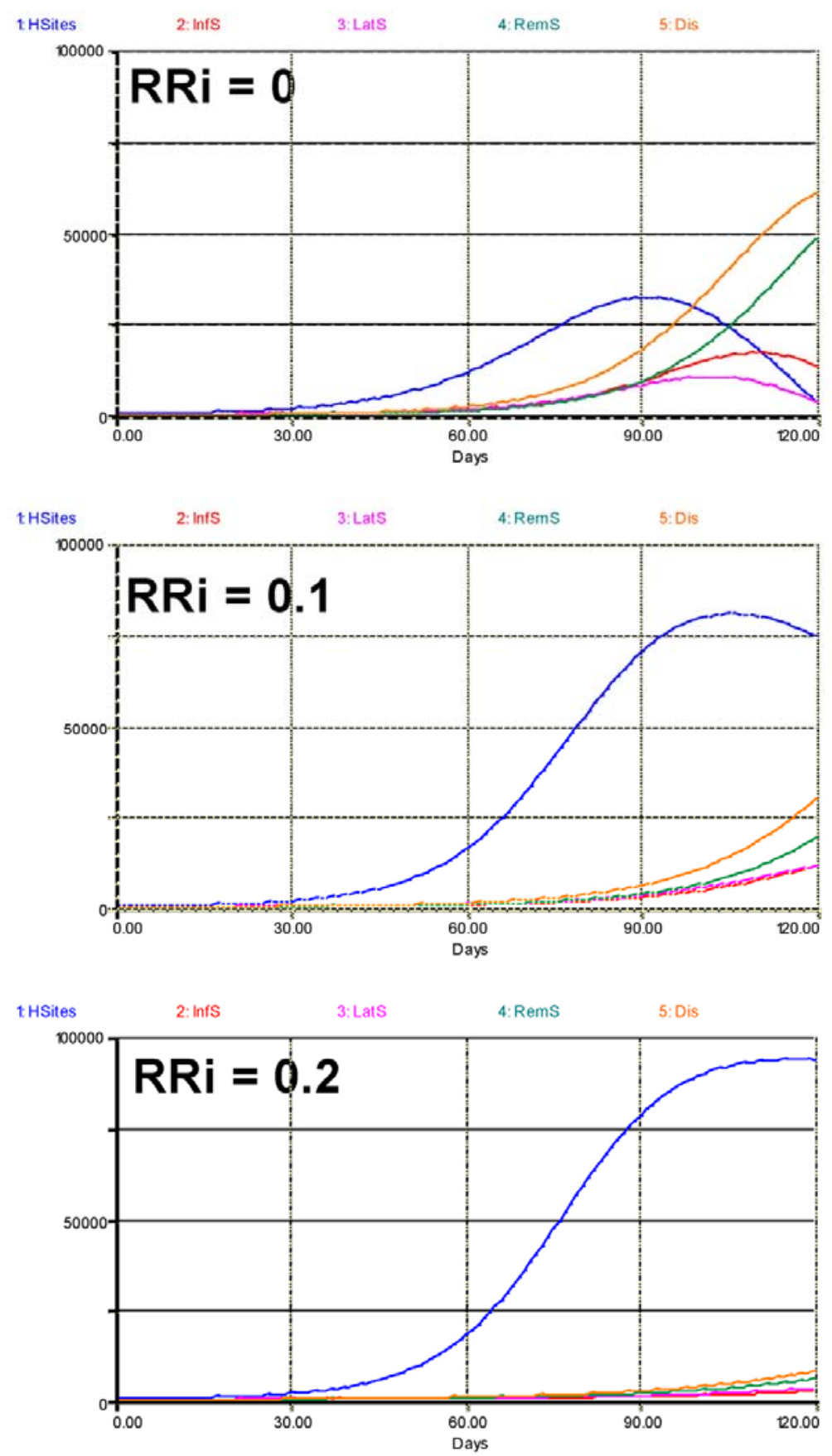

Figure 6.2. Overall simulated effects of increasing levels of components of partial resistance, where $\mathrm{RRIE}=\mathrm{RRSP}=\mathrm{RRLP}=\mathrm{RRIP}=\mathrm{RRi}$ are set to three values, 0, 0.1, and 0.2. 1: healthy sites; 2 : infectious sites; 3 : latent sites; 4 : sites removed from the epidemiological process; 5: accumulated visibly diseased sites (infectious and removed). Horizontal axis: time (days); vertical axis: numbers of sites. 
A second question concerns which component of resistance has the strongest suppressive effect, given the model structure we developed, and its underlying hypotheses. The outputs of such analysis are shown in Fig. 6.3, where each component was given a value of 0.2 in turn. The first run (top left) is identical to the simulation shown at the top of Fig. 6.2, where all components of resistance are null. The second run pertains to RRIE or RRSP, since any partial resistance on these two components have the same bearing within the chosen model structure. In that case, the terminal level of disease is not strongly reduced, but the rate of the epidemic is strongly reduced, with a disease progress curve becoming exponential, while it is logistic in the absence of resistance. The latent, infectious, and removed progress curves are also strongly reduced in their rates. A strong increase in crop growth also occurs. The effects of a partial resistance in the latent (RRLP) or infectious (RRIP) stages have graphically similar effects. Comparing the effects of an increased RRLP and RRIP, however, indicates a strong reduction in the terminal numbers of latent and infectious sites. If the simulation run were longer (e.g., 150 instead of 120 days; not shown), an increased RRLP leads to a near-complete exhaustion of sites, except for the postinfectious (removed) ones, and a collapse of the healthy sites; whereas an increased RRIP in effect delays the exhaustion of latent and infectious sites and, surprisingly, enables a renewed increase of healthy sites in the end of the run.
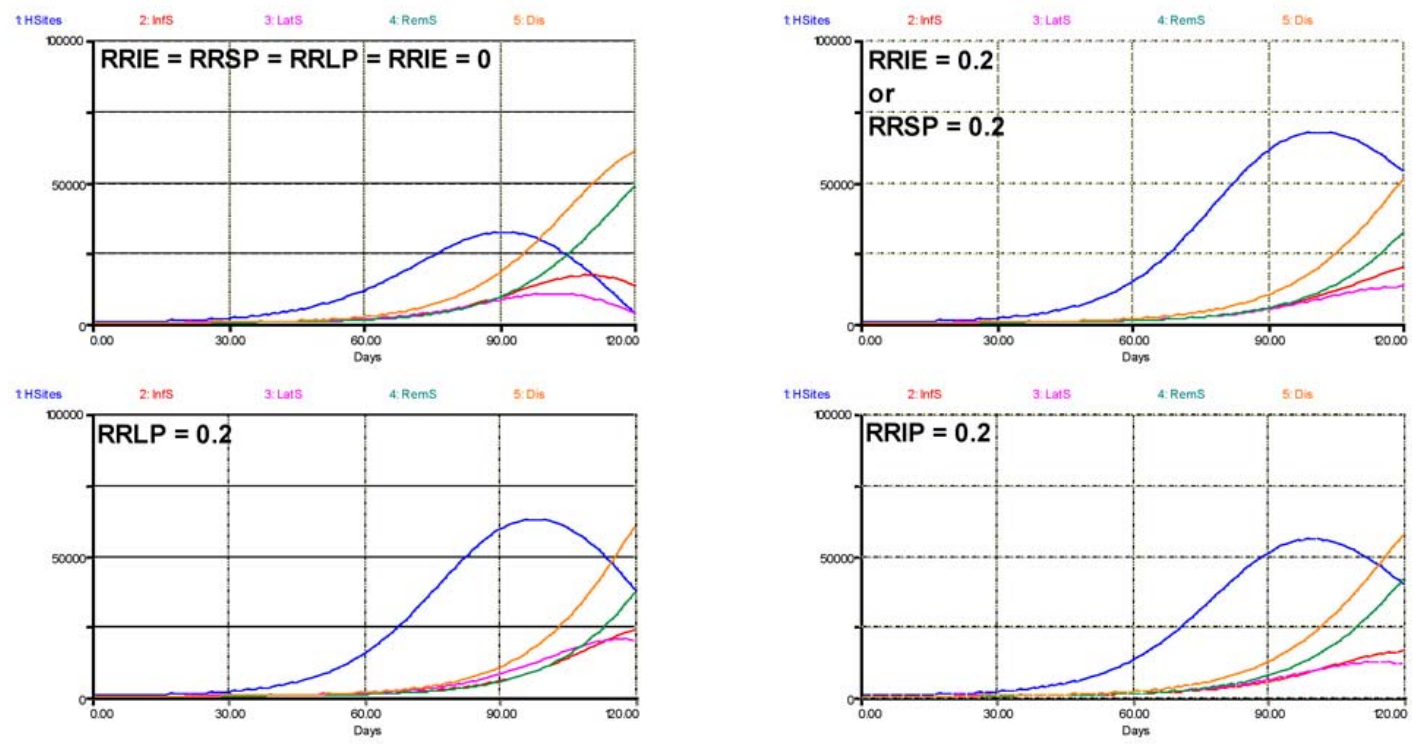

Figure 6.3. Simulated effects of individual components of resistance. 1: healthy sites; 2 : infectious sites; 3: latent sites; 4: sites removed from the epidemiological process; 5: accumulated visibly diseased sites (infectious and removed). Horizontal axis: time (days); vertical axis: numbers of sites. 


\section{Dealing with pathogen diversity}

Pathogen diversity is a fact, which accounts for, among many other things, resistance genes being overcome. For a long time, the process of resistance genes being overcome was considered to apply to qualitative resistance only: a qualitative (complete) resistance exerts such a selection pressure that isolates of the pathogens that can overcome the qualitative resistance emerge and replace the former pathogen genotypes. Could this be so in the case of partial resistance? In the beginning of this chapter, a brief overview of the diversity of resistances was given. One may consider that such a diversity also exists among pathogens (which may vary in terms of virulence or avirulence, or in degrees of aggressiveness), as well as among the mechanisms through which resistances may be overcome.

Let us consider a simplified case where only two isolates of the same pathogen occur.

Isolate 1 has the following characteristics:

- it represents 95 of the 100 initial infections at epidemic onset ( $\mathrm{t}=20$ days);

- the cultivated variety has a low level of partial resistance to isolate 1 , with RRIE $=$ RRSP $=$ RRLP $=$ RRIP $=0.1$

while isolate 2 has the corresponding characteristics:

- it represents only 5 of the 100 initial infections at epidemic onset;

- the cultivated variety has no partial resistance to isolate 2 .

The results of the simulations are shown in Fig. 6.4. In terms of proportion of isolates (expressed as 'visibly' diseased sites), the dynamics of isolate 2 of course is delayed.

Nevertheless, with a much stronger slope, the final number of sites diseased with isolate 2 is higher. This particular output illustrates again one of the major strengths of modeling; that is, its

ability to 'see' disease progress, whereas, of course, lesions caused by different isolates cannot be visually distinguished.

Crop growth is affected as in the previous outputs, with a sharp decline at the end of the run, while the removed sites accumulate.

The dynamics of the two isolates are quite different. The outputs at the bottom of Fig. 6.4 show the value of the rates of infection (INFECTION) of both isolates. While that of isolate 1 is initially high, it rapidly slows down, and tapers off. By contrast, the rate of infection of isolate 2 
is initially very low, then increases sharply to a much higher maximum, and declines too, but only at the end of the simulation, as the dual epidemic is running out of healthy sites. Similarly, the numbers of latent and infectious sites are much higher for isolate 1 than isolate 2 at the onset of the epidemic. If another simulation were executed using the final values of this run, isolate 1 would be displaced by isolate 2 , and the partial resistance would be overcome. Over successive seasons (or if the crop cycle were longer), this would lead to a population displacement, where the more aggressive strain of the pathogen would eventually dominate.
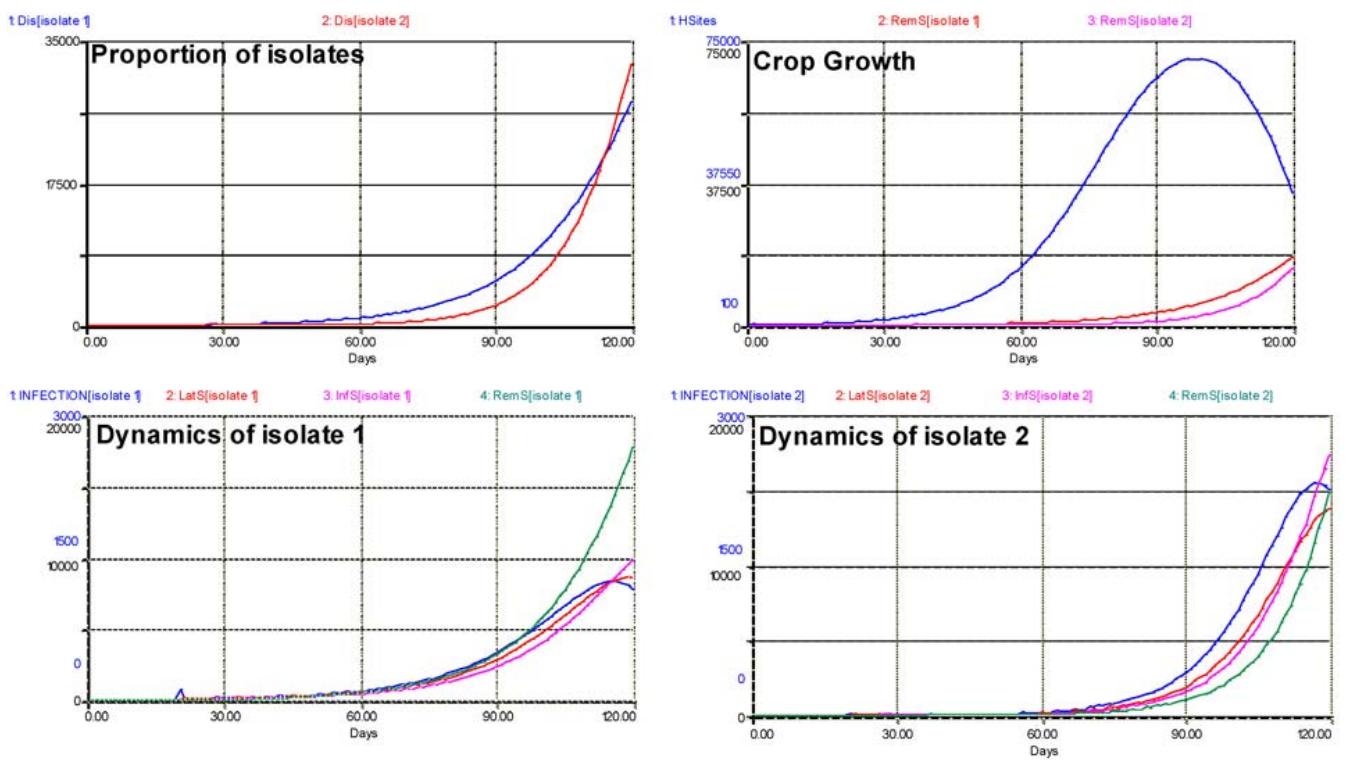

Figure 6.4. Dual dynamics of two isolates with differing adaptations to partial resistance. Note that the dynamics of isolates include the variation of the rate of infection over time. 1: healthy sites; 2 : infectious sites; 3 : latent sites; 4: sites removed from the epidemiological process; 5: accumulated visibly diseased sites (infectious and removed). Horizontal axis: time (days); vertical axis: numbers of sites.

\section{Perspectives and challenges}

The possibility of implementing integrative biology at the system level would represent a major scientific milestone. Progress in molecular biology is now such that never has this possibility been so real. Simulation modeling could play a key role in such advances, especially to breed durable host plant resistances. New genomic platforms in crop species, such as a nested association mapping populations, are providing unprecedented tools to identify quantitative trait 
loci (QTLs; Poland et al., 2009). Linking field ("phenotyping") data to molecular information through simulation modeling might become a reality (Yin et al., 2004), bridging several levels of integrations of life (from the gene to the individual plant, and to the plant population; de Wit and Goudriaan, 1978). This is fraught with difficulties, of course, but worth considering, for instance, through stepwise integration of sub-models. Difficulties might not lie only in the modeling, but in the use of suitable, reliable phenotyping data too (Parlevliet, 1979).

Challenges are also related to the nature of the host plant, or the pathosystem:

- $\quad$ some host crops may be more difficult to handle than others, making breeding programs more difficult;

- because of their seeds: working on cereals is far easier than on tuber crops, for instance;

- $\quad$ because of their life cycle - the life cycle of a cassava crop lies between 12 to 18 months (with no sexual reproduction occurring in the field); perennials are very difficult to address, not only because of their long life cycles, but also their complex genetic make-ups;

- partial resistance can be very hard to address in some pathosystems. Soil-borne and vector-borne pathogens were mentioned at the beginning of this chapter, thus difficulties arise from

- $\quad$ the complexity of the underlying system (vector-borne diseases); or difficulties in observing and measuring processes (soil-borne diseases), or both;

- and, ironically, some pathosystems are hard to address because they involve such a simple life-cycle of the pathogen. Such is the case of blights caused by, e.g.,

\section{Rhizoctonia solani.}

- the above problem may be compounded by the fact that epidemics are so strongly dependent on crop growth (they actually can be termed 'canopy-borne', even if the inoculum is not). This leads to the complex task of partitioning the effects of QTLs that affect plant habit, of QTLs that may influence partial resistance, or both (Srinivasachary et al., 2011). 


\section{Simulations}

The STELLA® model provided with this chapter (EPIDEMRES.STM) will allow you to explore the model structure and equations, and run the model with varying values of components of resistance, and see their effects on simulated epidemics.

\section{Summary}

- Simulation models can be used to address host plant resistance, especially quantitative host plant resistance.

- Simulation modeling can thus become a very powerful tool to phenotyping host plant resistance, because it allows one to track, over time, processes that cannot be seen, and can be linked with current advances in molecular breeding.

- A component of partial resistance (i.e., of quantitative resistance) is one independent element of a chain that contributes to suppressing, to some degree, disease progress.

- A component of partial resistance is equivalent to a dimensionless relative resistance coefficient, RR, which varies between 0 and $1: 0 \leq R R \leq 1$.

- Components of partial (quantitative) resistance can be defined, for instance for infection efficiency, spore production, infectious period and latency period: RRIE, RRSP, RRIP, and RRLP.

- Conversely, a relative (quantitative) resistance, $\mathrm{RR}_{\mathrm{c}}$, of a variety carrying several components of resistance can be calculated.

- These components can be implemented in the simulation model developed so far, and the effects of each component, alone or in combination, can be assessed.

- Pathogen diversity, in terms of varying levels of aggressiveness, can be addressed as well. An example of a variety with components of resistance confronted with two isolates differing in aggressiveness is given, where quantitative (partial) resistance is overcome, leading to population displacement by a more aggressive pathogen strain.

- A STELLA® model is attached, allowing users to run the model with varying values of components of resistance, and see their effects on simulated epidemics. 


\section{References}

Agrios, G.N. 2005. Plant Pathology, Fifth Edition. Elsevier Academic Press, Burlington, MA, USA.

Andrade-Piedra, J. L., Hijmans, R. J., Forbes, G. A., Fry, W. E., and Nelson, R. J. 2005.

Simulation of potato late blight in the Andes. I: Modification and parameterization of the LATEBLIGHT model. Phytopathology 95:1191-1199.

Bonman, J.M., Khush, G.S., and Nelson, R.J. 1992. Breeding for resistance to pests. Annual Review of Phytopathology 30:507-528.

Butt, D. J., and Royle, D. J. 1980. The importance of terms and definitions for a conceptually unified epidemiology. Pages 29-45 In: Comparative Epidemiology. A Tool for Better Disease Management. Palti, J. and Kranz, J. eds. Pudoc, Wageningen.

de Wit, C.T., and Goudriaan, J.G. 1978. Simulation of Ecological Processes. Pudoc, Wageningen, 175p.

Eversmeyer, M.G., and Kramer, C.L. 2000. Epidemiology of wheat leaf and stem rust in the central great plains of the USA. Annual Review of Phytopathology 38:491-513.

Flor, H.H. 1946. Genetics of pathogenicity in Melampsora lini. Journal of Agricultural Research 73:335-357.

Flor, H.H. 1971. Current status of the gene-for-gene concept. Annual Review of Phytopathology 9:275-296.

Gilligan, C. A. 1990. Mathematical modeling and analysis of soilborne pathogens. Pages 96-142 In: Epidemics of Plant Diseases, Second Edition J. Kranz ed. Springer-Verlag, Berlin.

Johnson, R. 1984. A critical analysis of durable resistance. Annual Review of Phytopathology 22:309-330.

Kranz, J. 1978. Comparative anatomy of epidemics. Pages 33-62 In: Plant Disease. An Advanced Treatise, Vol. IV. J.G. Horsfall and E.B. Cowling, eds. Academic Press, New York.

Kranz, J. 1990. Epidemics, their mathematical analysis and modeling: an introduction. Pages 111 In: Epidemics of Plant Diseases. Second Edition. J. Kranz, ed. Springer Verlag, Berlin. Madden, L. V., Jeger, M. J., and Van den Bosch, F. 2000. A theoretical assessment of the effects of vector-virus transmission mechanism on plant virus epidemics. Phytopathology 90:576594.

Padwick, G.W. 1956. Losses caused by plant diseases in the tropics. Comm. Mycological Inst. Kew, Surrey, Phytopath. Papers N¹, 60 p. 
Parlevliet, J. E., and Kuiper, H. J. 1977. Resistance of some barley cultivars to leaf rust, Puccinia hordei. Polygenic partial resistance hidden by monogenic hypersensitivity. Neth. J. Pl. Pathol. 83:85-89.

Parlevliet, J. E. 1977. Plant pathosystems: an attempt to elucidate horizontal resistance. Euphytica 26:553-556.

Parlevliet, J. E. 1979. Components of resistance that reduce the rate of disease epidemic development. Annual Review of Phytopathology 17:203-232.

Parlevliet, J. E., and Zadoks, J. C., 1977. The integrated concept of disease resistance: a new view including horizontal and vertical resistance in plants. Euphytica 26:5-21.

Poland, J. A., Balint-Kurti, P. J, Wisser, R. A., Pratt, R. C., and Nelson, R. J. 2009. Shades of gray: the world of quantitative disease resistance. Trends in Plant Science. 14:21-29.

Rapilly, F. 1979. Simulation d'une épidémie de Septoria nodorum Berk. sur blé. Etude des possibilités de résistance horizontale. Bull OEPP 8:243-250.

Robinson, R. 1976. Plant Pathosystems. Springer Verlag, Berlin, Heidelberg, New York, 184p.

Savary, S., Noirot, M., Bosc, J.P., and Zadoks, J.C. 1988. Peanut rust in West Africa: a new component in multiple pathosystem. Plant Dis. 78: 1001-1009.

Savary, S., De Jong, P.D., Rabbinge, R., and Zadoks, J.C. 1990. Dynamic simulation of groundnut rust, a preliminary model. Agricultural Systems 32: 113-141.

Savary, S., Nelson, A., Willocquet, L., Pangga, I., and Aunario, J. 2012. Modelling and mapping potential epidemics of rice diseases globally. Crop Protection 34: 6-17.

Srinivasachary, Willocquet, L., and Savary, S. 2011. Resistance to rice sheath blight (Rhizoctonia solani Kuhn) [teleomorph: Thanatephorus cucumeris (A.B. Frank) Donk.] disease: current status and perspectives. Euphytica 178:1-22.

Stokstad, E. 2007. Deadly wheat fungus threatens world's breadbaskets. Science 315, 1786-1787

Van der Plank, J.E. 1963. Plant Diseases. Epidemics and Control. Academic Press, New York.

Yin, X., Struik, P. C., and Kropff, M. J. 2004. Role of crop physiology in predicting gene-tophenotype relationships. Trends in Plant Science 9:426-432.

Zadoks, J. C. 1971. Systems analysis and the dynamics of epidemics. Phytopathology 61:600610.

Zadoks, J. C. 1972a. Methodology in epidemiological research. Annual Review of Phytopathology 10:253-276. 
Zadoks, J. C. 1972b. Modern concepts in disease resistance in cereals. Pages 89-98 In: The Way Ahead in Plant Breeding. F.A.G.H Lupton, G. Jenkins, and R. Johnson, eds. Proc. $6^{\text {th }}$ Cong. Eucarpia. Cambridge, 89-98.

Zadoks, J.C. 1977. Simulation models of epidemics and their possible use in the study of disease resistance. Pages 109-118 In: Induced Mutations Against Plant Diseases. IAEA, Ed. Vienna.

Zadoks, J. C., and Schein, R. D. 1979. Epidemiology and Plant Disease Management. Oxford University Press, New York. 427p.

Zadoks, J. C., and Van Leur, J. A. J. 1983. Durable resistance and host-pathogen-environment interaction. Pages 125-140 In: Durable Resistance in Crops. F. Lamberti, J. M. Waller, and N. A. Van der Graaff, eds. Plenum Publishing Corporation, New York.

\section{Exercises and questions}

\section{Questions}

1. A component of resistance
a. can take values above 1
b. can affect different processes of the monocycle
c. affects only one process of the monocycle
d. decreases when its effect on the monocycle processes increases

2. The different components of resistance
a. may have different effects on the simulated epidemics
b. have the same effects on the simulated epidemics
c. have an additive effect when combined
d. have a more than additive effect on epidemics when combined

3. Do components of resistance have a direct effect on yield?

\section{Answers}

1. c: affects only one process of the monocycle. 
2. a: may have different effects on the simulated epidemics, and d: have a more than additive effect on epidemics when combined.

3. No. Components of resistance slow epidemics down. They may have, through this reduction of disease, a strong effect on yield in some cases.

Appendix 6.1. Program listing of EPIDEMRES

HSites $(\mathrm{t})=$ HSites $(\mathrm{t}-\mathrm{dt})+(\mathrm{RCG}-\operatorname{INFECTION}-\mathrm{RSEN}) * \mathrm{dt}$

INIT HSites $=100$

INFLOWS:

$\mathrm{RCG}=\mathrm{RRCG} * \mathrm{HSites} *(1-(\mathrm{HSites} / \mathrm{MaxS}))$

OUTFLOWS:

INFECTION $=(($ IEcor $*$ SPcor $) *$ CORF $*$ InfS $)+$ INOCPRIM

RSEN $=$ REMOVAL

$\operatorname{InfS}(\mathrm{t})=\operatorname{InfS}(\mathrm{t}-\mathrm{dt})+($ TRANSFERT - REMOVAL $) * \mathrm{dt}$

INIT InfS = 0,0,0,0,0,0,0,0,0,0

TRANSIT TIME $=$ varies

INFLOW LIMIT $=$ INF

CAPACITY $=$ INF

INFLOWS:

TRANSFERT $=$ CONVEYOR OUTFLOW

TRANSIT TIME $=6 *$ LPcor

OUTFLOWS:

REMOVAL $=$ CONVEYOR OUTFLOW

TRANSIT TIME $=10 *$ IPcor

LatS $(\mathrm{t})=\mathrm{LatS}(\mathrm{t}-\mathrm{dt})+($ INFECTION - TRANSFERT $) * \mathrm{dt}$

INIT LatS $=0,0,0,0,0,0$

TRANSIT TIME $=$ varies

INFLOW LIMIT $=$ INF

CAPACITY $=$ INF 
INFLOWS:

INFECTION $=(($ IEcor $*$ SPcor $) *$ CORF $*$ InfS $)+$ INOCPRIM

OUTFLOWS:

TRANSFERT $=$ CONVEYOR OUTFLOW

TRANSIT TIME $=6 *$ LPcor

$\operatorname{RemS}(\mathrm{t})=\operatorname{RemS}(\mathrm{t}-\mathrm{dt})+(\operatorname{REMOVAL}) * \mathrm{dt}$

INIT RemS $=0$

INFLOWS:

REMOVAL $=$ CONVEYOR OUTFLOW

TRANSIT TIME $=10 *$ IPcor

$\mathrm{ACI}=\mathrm{LatS}+\mathrm{InfS}+\mathrm{RemS}$

$\mathrm{CORF}=1-(\mathrm{ACI} /(\mathrm{ACI}+\mathrm{HSites}))$

$\mathrm{DAY}=\mathrm{TIME}$

Dis $=\operatorname{InfS}+$ RemS

$\mathrm{IE}=0.3$

$\mathrm{IEcor}=\mathrm{IE} *(1-\mathrm{RRIE})$

INOCPRIM = IF $($ DAY=20) THEN 100 ELSE 0

IPcor $=1 *(1-$ RRIP $)$

LPcor $=1 /(1-R R L P)$

$\operatorname{MaxS}=100000$

$\mathrm{RRCG}=0.1$

RRIE $=0$

RRIP $=0$

RRLP $=0$

$\mathrm{RRSP}=0$

SPcor $=1-$ RRSP 


\section{Interlude: What Has Been Omitted So Far?}

The short answer to the above question is: quite a lot indeed. The purpose of the previous chapters was not to provide a comprehensive overview of methods and approaches in modern botanical epidemiology, which is provided in several references especially in Madden et al. (2007). What we tried instead is to provide a stepwise introduction to mechanistic simulation modeling, as one of the many methods available to botanical epidemiology. The choice of this approach was made because modeling attracts many plant scientists who, however, are deterred by the mathematics that might be involved. Note that much of the relationships we have used so far to analyze epidemiological systems are very simple. These relationships are closer to basic physical ones (thus the reference to dimensions in equations), rather than mathematical ones.

Yet many epidemiologists will rightly be prompt to point at the many gaps we left behind us. In the following elements, we briefly try to address at least some of these gaps from a practical modeling perspective. The points that are indicated below should not be seen as a check-list of gaps (there are more), but as a series of elements we feel are particularly important, especially the very last one.

\section{Patterns of epidemics}

Only one pattern of epidemics has been discussed in the prototype model developed from Chapter 4 onwards. This model implies that, in the course of a cropping season, each lesion produces a progeny of new lesions. Such epidemics are called polycyclic (e.g., Van der Plank, 1963; Zadoks and Schein, 1979). Many plant disease epidemics are not based on this pattern.

After all, the notion of successive, then overlapping, waves of sites that become infected, then infectious, and later removed is based on the quite anthropomorphic notions of a crop and of a cropping season. One may thus see a crop, from the (typical, but not sole) western point of view as a cohort, i.e., a population of plants whose development is nearly the same, having been established at the same time in a cultivated field. Many agricultural systems do not, however, consist of cohorts, nor are they homogeneous. In quite a few respects, they are thus closer to natural ecosystems. This point is briefly addressed below.

Some epidemics are caused by pathogens that produce only one wave (one generation) of propagules per season. Such epidemics are called monocyclic (e.g., Van der Plank, 1963; Zadoks and Schein, 1979). A typical example is that of some (specialized) diseases infecting flowers 
(Ngugi and Scherm, 2001). Such epidemics can be of extreme importance, such as ergot (Agrios, 2005; Zadoks, 2008). There are different reasons why epidemics might be monocyclic. One is that the host is susceptible for a very short period of time, such as in the case of some flower diseases (Ngugi and Scherm, 2001); but another reason can be that the pathogen produces so very few propagules that only one wave of infection occurs in the course of a cropping season. The latter case prompted Zadoks and Schein (1979) and Ngugi and Scherm (2001) to compare such pathogens to K-strategists, as opposed to r-strategists, which cause polycyclic diseases, to bring us back to classical ecological concepts (May and McLean, 2007).

\section{Confusing patterns of epidemics}

Many diseases caused by soil-borne pathogens have been, and still are, often perceived as monocyclic diseases. This, actually, is often not true: even if there are few infections, even if the distance range of new infections from the mother lesion is small, many soil-borne diseases actually are polycyclic (e.g., Willocquet et al., 2008). In a key article, Pfender (1982) clarified the difference between deriving a model from the known biology of the disease, and the opposite: "the error [is] that the nature of the disease cycle is being inferred from the disease progress curve. The nature of the disease must be determined before an appropriate model can be chosen." This emphasizes the importance of focusing on processes and modeling them, as opposed to describing, even numerically, phenomena. In other words, the emphasis is on the importance of deriving models that truly have biology-related parameters (vs., for example, statistical models).

\section{Many epidemic patterns may be considered}

From an evolutionary perspective, one might admit that the notions of polycyclic or monocyclic epidemics are rather academic. As with any organism (Dodds, 2009), the point of view of the pathogen is to (1) survive, (2) multiply, (3) spread, and, in so doing, (4) adapt. Whether these processes happen during one or several cropping seasons, in fields cultivated with one or several plant species, in environments that are temporally and/or spatially uniform, does not really matter: failing to achieve any one of these steps means extinction. Phrased otherwise, the spatiotemporal diversity of agroecosystems is the context where these four events must take place; conversely, the spatiotemporal diversity of agroecosystems may, in some cases, be manipulated so that epidemics can be suppressed. 
Let us consider the successive growing seasons of a vegetable crop, and a soil-borne disease, caused by, for example, Rhizoctonia solani. Let us further assume that the epidemic is monocyclic during a given growing season. The very fact that seasons follow after seasons will lead individual monocyclic epidemics to be concatenated in what was termed by Zadoks and Schein (1979) a polyetic epidemic. Each epidemic contributes to increasing the stock of soilborne primary inoculum. Modeling this accumulation, its remobilization, and the effects of various crop management components, represents a very useful exercise to both understand and manage a difficult case. Difficulties arise due to few options for disease management, the pathogen has an extremely wide host range, and vegetable growers cannot easily shift to new lands, especially in peri-urban areas where vegetable growing is a most profitable activity. Such polyetic epidemics have so far been very poorly studied (e.g., Amorim and Bergamin Filho, 1991), but one might want to consider them as a particular form of polycyclic epidemic, characterized by having, at the beginning of each growing season (1) a new cohort of host plant (or host tissues, for perennials) is established and (2) the pathogen population has been reduced, and/or underwent a phase during which it had to survive without hosts.

We believe that complicated models do not need to be developed to gain a better understanding of patterns in complex systems. The case of household gardens highlights this point well. Household gardens are very important systems, agriculturally, socially, culturally, and from the point of view of sustainable food security, both in the developing and the developed worlds (Niñez, 1987). At times of economic and food crises, such systems may play a critical role. Plant pathology, and in general, epidemiology, ecology, and agronomy (as well as the Social Sciences and Medicine), might both have an important role to play, as well important lessons to learn, in household gardens. These systems often are very complex (e.g., Conway, 1994); yet, we believe that much insight could still be gained using un-complicated models.

The landscapes where epidemics occur may provide opportunity, or hindrance, to disease spread. This has been at the center of fundamental thinking for decades. For instance, Heesterbeek and Zadoks (1987) devised the notions of order 0 (focal), order 1 (general), and order 2 (pandemics) epidemics. In agricultural systems such as the Mekong Delta of Vietnam or the Central Plain of Thailand, up to seven rice crops are grown over two years, providing "green bridges" for pathogens to spread from one older cohort (field) to a younger one. The epidemiology of plant diseases in such environments has been studied in much detail, especially with respect to viral diseases, and the models derived by Chancellor, Azzam, and Holt (Azzam 
and Chancellor, 2002; Chancellor et al., 2006) exemplify a success story based on clear understanding of processes, their quantification, their implementation in models, and leading to true application to disease management at the farm and landscape scales.

\section{The critical importance of space-time interactions}

The so-called 'mean field' hypothesis has been mentioned across the successive examples discussed so far. In other words: all the model structures discussed so far implicitly assumed that each healthy site, at any point of time, was accessible to infection. Conversely, they also implied that each propagule, be it for instance a viruliferous vector or a bacterial spore, had the same chance to infect, meaning a propagule that has been through the sequence of dispersal steps successfully, and has been lucky enough to (1) not land on bare ground, or on an unsuitable host, or in a hostile soil environment, and (2) encounter a site which is not already infected (i.e., a very lucky propagule indeed). The 'mean field' hypothesis is suitable for some thinking, but is admittedly inadequate for many systems.

Spatial and temporal processes cannot be distinguished and represent a mainstream area of investigation in Ecology (e.g., Renshaw, 1991; May and McLean, 2007), and in Botanical Epidemiology (Madden et al., 2007). A keystone article by Jeger (1982) provided a first analytical framework of plant disease epidemics in time and space.

Each epidemic is a unique event. We have not (and will not) speak of stochasticity in this module. Xu and Ridout (1996) demonstrated in a very elegant way how the inherent variability in the dispersal characteristics of a pathogen, and to a lesser degree, of the initial conditions of the modeled pathosystem (amount of primary inoculum) may affect the final spatial distribution and the final amount of disease. Being stochastic, their model simulates epidemics as unique successions of dispersal and infection events. Simulation outputs show expanding foci as propagules are dispersed and as epidemics unfold, sometimes giving rise to secondary foci, or, on the contrary, to general epidemics. Their model, in short, matches reality so well that is shows how difficult accurately measuring disease can be.

This transition chapter in neither the right place for expanding on this subject, nor on several other aspects we have left behind. Let us simply put it this way: the spatial structure of epidemics is very closely associated to their temporal ones. The spread (or extensification) of disease in the host population goes hand in hand with its local multiplication (intensification). The respective weights of extensification and intensification depend on the pathosystem 
considered. Without extensification, the pathogen would locally intensify at one or a few sites, rapidly reach a local carrying capacity, and die. Without intensification, the pathogen would simply be unable to reproduce itself. From the pathogen viewpoint, extensification implies risks

- from total propagule loss (e.g., deposition on the ground) to the encounter of unsuitable or resistant hosts; but it also entails opportunities: encountering more susceptible host sites. Aggregated (focal) epidemics lead to low levels of terminal disease, whereas random (general) epidemics lead to high levels of terminal disease (Xu and Ridout, 1996). Further, and critically, extensification offers the chance to the pathogen to evolve and adapt to different genotypes of its host. This is particularly true in complex agrosystems (Lenné and Jeger, 1994).

\section{References}

Agrios, G. N. 2005. Plant Pathology, 5th ed. Elsevier Academic Press Publications, Burlington, MA.

Anderson, E. 1952. Plants, Man, and Life. Little, Brown \& Co., Boston.

Azzam, O., and Chancellor, T. C. B. 2002. The biology, epidemiology, and management of rice tungro disease in Asia. Plant Dis. 86:88-100.

Amorim, L., and Bergamin Filho, A. 1991. Sugarcane smut development models: II. Polyetic curves of disease progress. Z. Pflanzenkrank. Pflanzensch. 98:613-618.

Chancellor, T. C. B., Holt, J., Villareal, S., Tiongco, E. R., and Venn, J. 2006. Spread of plant virus disease to new plantings: a case study of rice tungro disease. Adv. Virus Res. 66:1-29.

Conway, G. R. 1994. Sustainability in agricultural development: trade-offs between productivity, stability, and equitability. J. Farm. Syst. Res. Ext. 4:1-14.

Dodds, W. K. 2009. Laws, Theories, and Patterns in Ecology. University of California Press, Berkeley, CA.

Heesterbeek, J. A. P., and Zadoks, J. C. 1987. Modelling pandemics of quarantine pests and diseases: problems and perspectives. Crop Prot. 6:211-221.

Jeger, M. J. 1983. Analysing epidemics in time and space. Plant Pathol. 32:5-11.

Lenné, J. M., and Jeger, M. J. 1994. Evaluation of plant pathogens in complex ecosystems. Pages 63-77 in: Ecology of Plant Pathogens. J. P. Blakeman, and B. Williamson, eds. CABI, Wallingford.

Madden, L. V., Hughes, G., and Van den Bosch, F. 2007. The Study of Plant Disease Epidemics. The American Phytopathology Press, St Paul, MN. 
May, R., and McLean, A., eds. 2007. Theoretical Ecology. Principles and Applications, 3rd ed. Oxford University Press, Oxford.

Ngugi, H. K., King, S. B., Holt, J., and Julian, A. M. 2001. Simultaneous temporal progress of sorghum anthracnose and leaf blight in crop mixtures with disparate patterns. Phytopathology 91:720-729.

Ngugi, H. K., and Scherm, H. 2006. Biology of Flower-Infecting Fungi. Annu. Rev. Phytopathol. 44:261-282.

Niñez, V., 1987. Household gardens: theoretical and policy considerations. Agric. Syst. 23:167186.

Pfender,W. F. 1982. Monocyclic and polycyclic root diseases: distinguishing between the nature of the disease cycle and the shape of the disease progress curve. Phytopathology 72:31-32.

Renshaw, E. 1991. Modelling Biological Populations In Space and Time. Cambridge University Press, Cambridge.

Van der Plank, J. E. 1963. Plant Diseases. Epidemics and Control. Academic Press, New York.

Willocquet, L., Lebreton, L., Sarniguet, A., and Lucas, P. 2008. Quantification of within-season focal spread of wheat take-all in relation to pathogen genotype and host spatial distribution. Plant Pathology 57:906-915.

Xu, X. M., and Ridout, M. S. 1996. Analysis of disease incidence data using a stochastic spatialtemporal simulation model. Asp. Appl. Biol. 46:155-158.

Zadoks, J. C. 2008. On the Political Economy of Plant Disease Epidemics - Capita Selecta in Historical Epidemiology. Wageningen Academic Publishers, Wageningen.

Zadoks, J. C., and Schein, R. D. 1979. Epidemiology and Plant Disease Management. Oxford University Press, New York.

\section{Suggested reading}

Bascompte, J., and Solé, R. V., eds. 2007. Modeling Spatiotemporal Dynamics in Ecology. Springer-Verlag, Berlin, Heidelberg.

Boudreau, M. A., and Mundt, C. C., 1994. Mechanisms of alteration in bean rust development due to intercropping, in computer-simulated epidemics. Ecol. Applic. 4:729-740.

Garrett, K. A., and Mundt, C. C. 1999. Epidemiology in mixed host populations. Phytopathology 89:984-990. 
Luo Yong, and Zadoks J. C. 1992. A decision model for variety mixtures to control yellow rust on winter wheat. Agric. Syst. 38:17-33.

Lannou, C., de Vallavieille-Pope C., and Goyeau, H. 1995. Induced resistance in host mixtures and its effect on disease control in computer-simulated epidemics. Plant Pathol. 44:478-489.

Jeger, M. J., Pautasso, M., Holdenrieder, O., and Shaw, M. W. 2007. Modelling disease spread and control in networks: implications for plant sciences. New Phytol. 174:279 -297.

Mundt, C. C. 1989. Modeling disease increase in host mixtures. Pages 150-181in: Plant Disease Epidemiology, vol 2. Genetics, Resistance, and Management. K. J. Leonard and W. E. Fry, eds. McGraw-Hill, New York.

Ngugi, H. K., King, S. B., Holt, J., and Julian, A. M. 2001. Simultaneous temporal progress of sorghum anthracnose and leaf blight in crop mixtures with disparate patterns. Phytopathology 91:720-729.

Savary, S., Noirot, M., Bosc, J. P., and Zadoks, J. C. 1988. Peanut rust in West Africa: a new component in multiple pathosystem. Plant Dis. $78: 1001-1009$.

Wolfe, M. S. 1985. The current status and prospects of multiline cultivars and variety mixtures for disease resistance. Annu. Rev. Phytopathol. 23:251-273. 


\section{Modeling Crop Losses}

In this short introductory section we want to ask ourselves: what is the use of simulation modeling in crop loss understanding and analysis? But before moving into the field of crop loss modeling, we need to point out a few elements.

- Production situations differ widely across world agroecosystems and are undergoing unprecedented changes (Dyson, 1999; WRI, 2005). One definition for 'production situation' refers to a hierarchy of constraints to crop production: from production level 1 , where no limit to crop growth occurs, and is defined only by radiation and plant genotype; to production level 2, where some water shortage occurs at least for some time of the growing season; to production level 3, where a shortage of nitrogen may also occur; and to production level 4, where the above limiting factors are compounded by shortages of phosphorus and/or potassium (Penning de Vries, 1982; Rabbinge et al., 1989). While this first definition focuses on crop production, another, holistic definition of a production situation is the 'body of environmental factors - physical, biological, social, and economic - where agriculture takes place' (De Wit, 1982). While both definitions have value, the latter enables one to link attainable yields to production situations, as well as to human components such as farmers' skills, socio-economic factors, and available techniques for future improvement (Van Ittersum and Rabbinge, 1997), and decisions (McRoberts et al., 2011).

- Crop losses caused by diseases, animal pests, and weeds, range between 20 to $40 \%$ of the yield that would be attained otherwise (e.g., Savary et al., 2005; Oerke, 2006), depending on the production situations. This obviously represents a massive challenge to food security and food safety, and cannot be ignored.

- Why do we address epidemiological and crop loss modeling in separate sections in this module? There are four reasons for this.

1. First, the linkage between epidemics and crop losses is that of a growing crop. This implies that we shall need to consider some basic agrophysiological principles.

2. A second reason is that the linkages epidemics-crop losses, on the one hand, and crop losses-economic losses, on the other hand, are non linear. The relationships between epidemics and crop losses are represented by damage functions, while the relationships between crop losses and economic losses are governed by loss functions (Zadoks, 1985). Thus, the translation of epidemics into crop losses is not direct, and in fact, is quite complex. 
3. A third reason is that we want to keep our models as simple and tractable as possible: we want to model for a purpose (Loomis et al., 1979): epidemics on the one hand, crop losses on the other. Linking the two implies complex feed-backs and feed-forwards, which, despite their relevance, would detract us from our main objective of providing introductory working elements to the topic.

4. Lastly, when thinking of crop losses, other harmful agents must be considered in addition to plant pathogens: animals (especially insects and spiders), and weeds. As a result, the analysis of crop losses entails the consideration of injuries caused by an array of crop harmful organisms. We shall see in the following chapters that considering so many different organisms does not lead to increased complexity.

- Crop losses — not epidemics — are the basis for disease management. As a result, the last three elements of the above imply a crucial issue: understanding and modeling crop losses is not easy. In particular, thresholds for decisions, whether strategic (before a crop is being established) or tactical (when the crop is standing) are not static, but variable (Zadoks, 1985; Rabbinge et al., 1989). One of the several causes for threshold variation is the considered production situation.

- Both damage and loss functions are highly variable (e.g., Zadoks, 1985; Savary et al., 2000), for a number of reasons, among which are (1) the nature of the harmful organism, and (2) the considered production situation (and therefore, the considered attainable, uninjured, yield). What follows does not dwell on this important topic. Simulation modeling, however, can be seen as one tool to address it.

Much of what will be developed in the following chapters is derived, with some expansion, but also some simplifications, from the book by Rabbinge et al. (1989). As in the previous chapters, our aim is to bring forward ideas and methods that can be implemented with ease, and we shall try to provide frameworks that are as simple as possible.

Let us try, at least temporarily, to answer the question we posed at the beginning of this introduction. Simulation modeling in crop loss analysis is useful in at least two important areas: one is to produce estimates of likely crop losses caused by one (sometimes several) yield reducers; another is to assess and rank the importance of yield reducers in terms of crop losses. There are other possible applications of simulation modeling. One potential application, which was widely shared when simulation modeling was new to the field of plant protection, was that it could become a tool to guide crop protection. There has been accumulating evidence, however, that this approach was unlikely to bear fruit for a number of reasons (e.g., Butt and Jeger, 1985; 
Jeger, 2000). Conversely, much simpler approaches based on very solid science could be far more successful, such as the EPIPRE program (Zadoks, 1989).

The second area where simulation modeling is a powerful tool is that, being processbased, it enables one to not only address "what has been lost", but also what could be gained. In other words, the approach enables one to explore scenarios where new crop health management approaches (e.g., new genotypes, different crop rotations) would be implemented. This is possibly the most exciting reason for using simulation modeling in this area.

\section{References}

Butt, D. J., and Jeger, M. J. 1985.The practical implementation of models in crop disease management. Pages 207-230 in: Advances in Plant Pathology, vol. 3. C. A. Gilligan, ed. Academic Press, London.

Dyson, T. 1999. World food trends and prospects to 2025. Proc. Natl. Acad. Sci. USA 96:59295936.

van Ittersum, M. K., and Rabbinge, R. 1997. Ecology for analysis and quantification of agricultural input-output combinations. Field Crops Res. 52:197-208.

Jeger, M. J. 2000. Bottlenecks in IPM. Crop Prot. 19:787-792.

Loomis, R. S., Rabbinge, R. and Ng, E. 1979. Explanatory models in crop physiology. Annu. Rev. Plant Physiol. 30:339-367.

McRoberts, N., Hall, C., Madden, L. V., and Hughes, G. 2011. Perceptions of disease risk: from social construction of subjective judgments to rational decision making. Phytopathology 101:654-665.

Oerke, E. C. 2006. Crop losses to pests. J. Agric. Sci. 144:31-43.

Penning De Vries, F. W. T. 1982. Systems analysis and models of crop growth. Pages 9-19 in: Simulation of Plant Growth and Crop Production. F.W.T. Penning de Vries, and H. H. Van Laar, eds. Pudoc, Wageningen.

Penning De Vries, F. W. T., and Van Laar, H. H., eds. 1982. Simulation of Plant Growth and Crop Production. Pudoc, Wageningen.

Rabbinge, R., Ward, S. A., and Van Laar, H.H., eds. 1989. Simulation and Systems Management in Crop Protection. Pudoc, Wageningen.

Savary, S., Willocquet, L., Elazegui, F. A., Castilla, N. P., and Teng, P. S. 2000. Rice pest constraints in tropical Asia: quantification of yield losses due to rice pests in a range of production situations. Plant Dis. 84:357-369. 
Savary, S., Teng, P. S., Willocquet, L., and Nutter, F. W., Jr. 2005. Quantification and modeling of crop losses: a review of purposes. Annual Review of Phytopathology 44:89-112.

de Wit, C. T. 1982. L'analyse des systèmes de production primaires. Pages 275-283 in: La Productivité des Pâturages Sahéliens. Une Etude des Sols, des Végétations et de l'Exploitation de cette Ressource Naturelle. F.W.T. Penning de Vries and M. A. Djiteye, eds. Agricultural Research Report 918, Pudoc, Wageningen.

World Resource Institute. 2005. Millenium Ecosystem Assessment: Ecosystems and Human Well-Being - Synthesis. Island Press, Washington, D.C.

Zadoks, J. C. 1985. On the conceptual basis of crop loss assessment: the threshold theory. Annu. Rev. Phytopathol. 23:455-473.

Zadoks, J. C. 1989. EPIPRE, a computer-based decision support system for pest and disease control in wheat: its development and implementation in Europe. Pages 3-29 in: K. J. Leonard and W. E. Fry, eds. Plant Disease Epidemiology, vol. 2, Genetics, Resistance, and Management. McMillan, New York.

\section{Suggested reading}

Cassman, K. G., and Woods, S., Coordinating Lead Authors. 2005. Cultivated Systems. Pages 741-789 in: Ecosystems and Human Well Being, Millenium Ecosystem Assessment, Current State and Trends, Vol. 1. Island Press, Washington, D.C.

Evans, L. T. 1998. Feeding the Ten Billions. Plants and Population Growth. Cambridge University Press, Cambridge.

Rossing, W. 1991. Simulation of damage in winter wheat caused by the grain aphid Sitobion avenae. 2. Construction and evaluation of a simulation model. Neth. J. Plant Pathol. 97:2554.

Rossing, W. 1991. Simulation of damage in winter wheat caused by the grain aphid Sitobion avenae. 3. Calculation of damage at various attainable yield levels. Neth. J. Plant Pathol. 97: 87-103.

Rossing, W. 1993. On damage, uncertainty and risk in supervised control. Aphids and brown rust in winter wheat as an example. PhD Dissertation, Wageningen Agricultural University.

United Nations Environment Program, 2007. GEO4 - Global Environment Outlook: Environment for Development. Progress Press, Malta. 


\section{Chapter 7. Crop Growth Modeling - Introducing GENECROP as a Framework}

Modeling the effects of injuries caused by pests (diseases, insects, and weeds) on crop growth and yield requires, as a first stage, the modeling of growth and yield of a crop in absence of injuries. This chapter will take you through the main processes involved in crop growth, how these processes are represented in a quantitative and dynamic manner, and how they are implemented into a simple model, which you can explore and run. This chapter starts with the RI-RUE paradigm, which has strong connections with the modeling of yield losses, and provides a very simple and robust framework to address crop growth under a set of biophysical constraints.

\section{The RI-RUE concept}

$R I$ is the radiation intercepted by the crop canopy and can be written in a simple way using Beer's law (Monsi and Saeki, 1953, see also, Goudriaan and van Laar, 1994; Whisler et al., 1986; Thornley and France, 2007) as:

$$
R I_{t}=R A D_{t} \times\left(1-e^{-k \times L A I_{t}}\right)
$$

where $R A D$ is the global solar radiation, that is, the incident radiation above the canopy; $k$ is the extinction coefficient; and LAI is the Leaf Area Index (i.e., the area of leaf per area of ground soil, dimension $=\left[\mathrm{L}^{2} \cdot \mathrm{L}^{-2}\right]$ with units $\left.\mathrm{m}^{2} \cdot \mathrm{m}^{-2}\right)$. The parameter $k$ depends on the direction of the radiation and on the orientation of the leaves. Average values for canopies with erect and horizontal leaves are about 0.6 and 0.8, respectively (Penning de Vries et al., 1989). The intercepted radiation increases with LAI, but the rate of increase diminishes as LAI increases. For a given value of LAI, the intercepted radiation is larger for canopies with horizontal than erect leaves (Fig. 7.1).

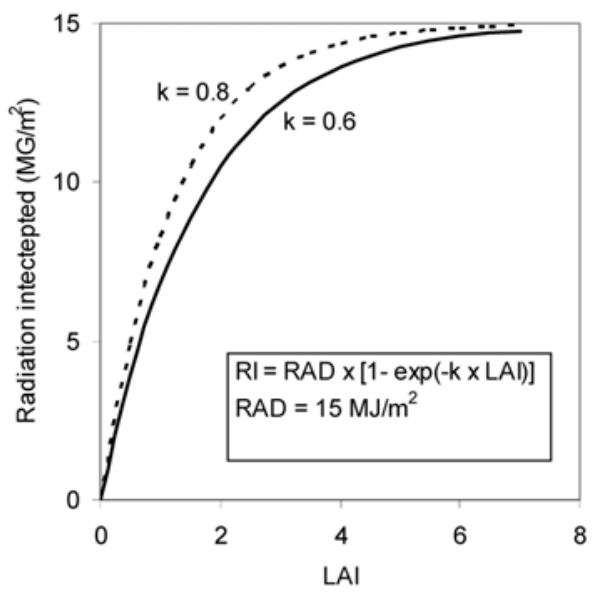

Figure 7.1. Relationships between radiation intercepted and LAI. 
Monteith (1977) established a robust, linear relationship between the accumulated crop biomass and $R I$. He introduced the concept of Radiation Use Efficiency $(R U E)$, which can empirically be estimated as the slope value from the linear regression of accumulated biomass over accumulated $R I$. $R U E$ represents the conversion of radiation energy into biomass. In other words, $R U E$ represents the amount of dry biomass (DBM) produced per unit of radiation energy intercepted by the crop canopy. An $R U E$ value for crops under non-limiting conditions is about $1.4 \mathrm{~g} \cdot \mathrm{MJ}^{-1}$, or approximately $2.8-3.2$ $\mathrm{g} \cdot \mathrm{MJ}^{-1}$ of photosynthetically active radiation (PAR; Monteith, 1977). Using Monteith's framework, the accumulated dry biomass over a time interval $[0, \mathrm{t}],\left(D B M_{t}\right)$ can therefore be written as:

$$
D B M_{t}=\int_{0}^{t} R I_{t} \times R U E_{t} \times d t
$$

This framework establishing relationships between crop growth, radiation interception, and $R U E$ has been used in a very wide range of examples to model crop growth in a simple way (e.g., Johnson et al., 1986; Sinclair, 1986; Steer et al., 1993; Richter et al., 2001; Kiniri et al., 2004). This framework is used here because it has also enabled addressing the effects of harmful organisms on crop growth in a generic, synthetic manner (see Chapter 8).

Main processes involved in crop growth captured into a simple crop growth simulation model for attainable growth and yield - GENECROP

\section{Model system and structure}

The time step of the GENECROP model is one day, and the system modeled is $1 \mathrm{~m}^{2}$ of crop. The different processes determining crop growth are embedded into the model in three components, which deal with: (1) the dynamics of crop development, (2) the accumulation of crop biomass, and (3) the growth in numbers of tillers (or shoots). A complete listing of the program can be found in Appendix 7.1.

The model structure can be explored by opening the STELLA model GENECROP.STMX and clicking on the "explore model" button. The model equations can be viewed by selecting the

"equation" level on the left part of the panel when opening the file. The flowchart of the model is given in Fig. 7.2. 

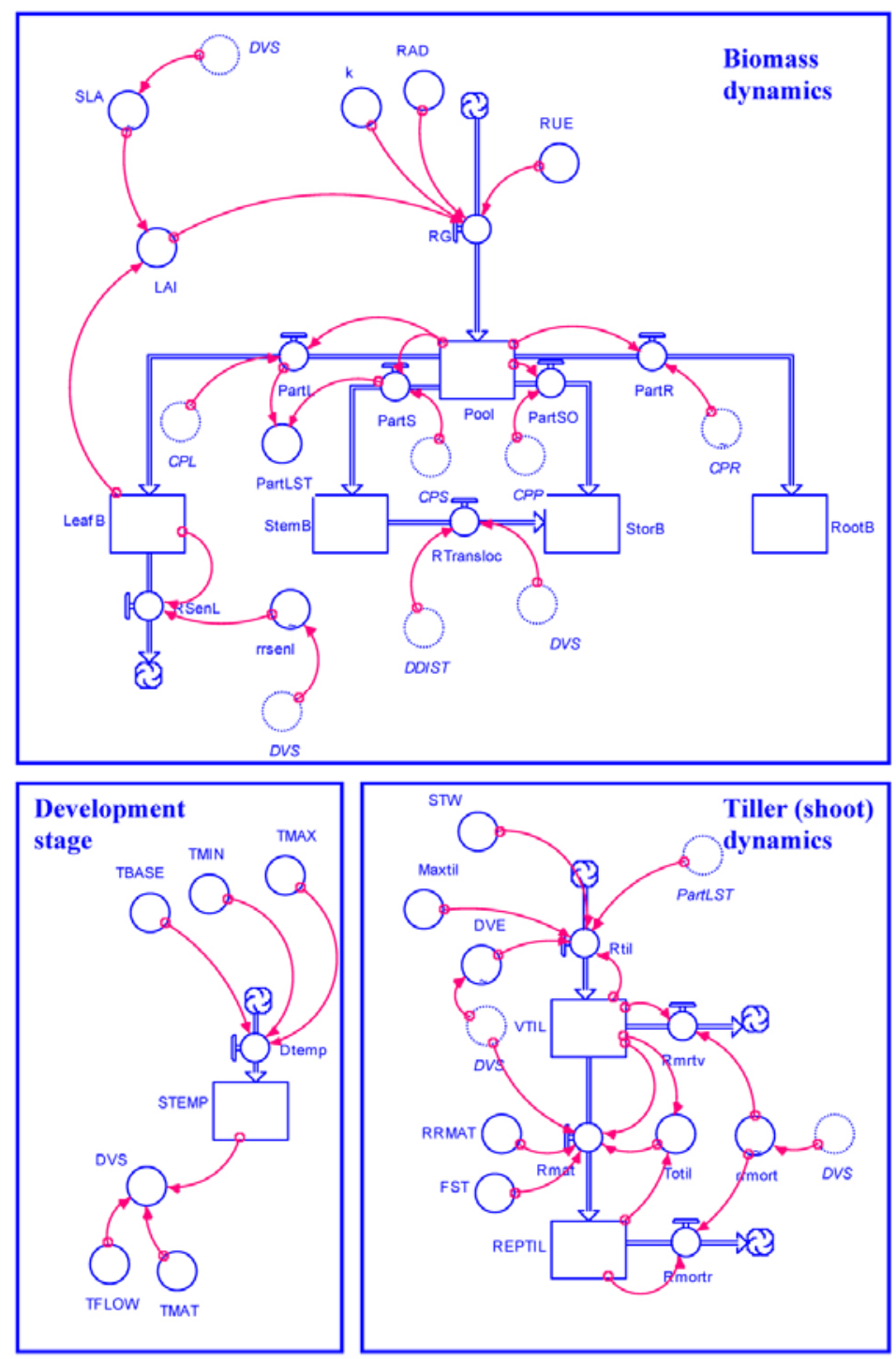

Figure 7.2. Flowchart of GENECROP, a generic model for attainable crop growth.

The main processes involved over the course of a crop cycle are: photosynthesis, biomass partitioning in growing plants, leaf senescence, and yield build-up. Most of these processes are driven by crop development.

As discussed in the earlier parts of this module, the level of detail in modeling which is required to simulate and understand the behavior of a system needs to be carefully pondered. This principle applies here, where these processes need to be seen from the point of view of our needs (modeling crop growth so that injuries caused by pests can be accounted for, and, eventually yield losses, computed), and to our ability (to what extent shall we be able to parameterize the processes induced by injuries caused by pests?). Crop growth modeling is a field of research and application in its own right, in which we cannot enter in detail here. Some references are given at this end of this 
chapter for the interested reader. Lines of investigation in this field (some of which are very current) include:

- the root/shoot relationships

- the remobilization of carbohydrates as harvestable organs grow

- the detailed analysis of nutrient and water efficiencies in their contribution to growth

- the physiological effects of symbionts in enhancing crop growth

- the interaction between plants in heterogeneous systems (inter- and intra-specific diversity)

- the use of models as aids to chart the path of molecular or conventional breeding (e.g., Yin et al., 2004)

The choice was made in this chapter to focus on a structure that retains the key elements of a simplified system (a growing crop stand), that nevertheless allows one to quantitatively account for the effects of crop harmful organisms. Therefore, the processes accounted for and their level of detail have been included in GENECROP so that (1) they can be represented in the simplest possible way, while (2) being able to include the different damage mechanisms by which crop growth and yield are affected by disease (pest) injuries.

The flowchart in Fig. 7.2 may seem rather complicated to the unaccustomed eye. Yet this flowchart, and the system it represents, actually is based on quite a limited series of processes, which have been used in a large number of crop systems:

1. Crop growth occurs. It depends on the amount of radiation, which the canopy is able to intercept at any level of its growth (over time, crops intercept very little, then quite a lot, and progressively less radiation as leaves are successively very young, fully established, and senescing).

2. The intercepted radiation is converted into carbohydrates through photosynthesis.

3. The accumulated carbohydrates are partitioned towards the different organs of a growing crop: leaves, roots, stems, and storage (harvestable) organs.

4. This partitioning process is dependent on the development stage of a crop. Young crops will allocate much of their early "earnings" through photosynthesis to roots and leaves; later-on, stems and leaves will become important investment organs; and towards the end of a crop cycle much of the photosynthates will be allocated to the storage (harvestable) organs. In other words, the growth of different organs is made dependent upon development. Physiological development in turn is made (as is often done) dependent on temperature.

5. For the sake of tracking the dynamic effects of damage mechanisms, a small set of variables are introduced to monitor the dynamics of shoots (or tillers, in the case of a cereal crop). 
The different symbols used in GENECROP and their meanings are shown in Table 7.1.

Table 7.1. Variables, acronyms, and examples of units in the GENECROP crop growth simulation model

\begin{tabular}{|c|c|c|c|}
\hline Variable type & Acronym & Meaning & Units \\
\hline \multirow[t]{8}{*}{ State variables } & LEAFB & Leaf biomass & $\mathrm{g} \cdot \mathrm{m}^{-2}$ \\
\hline & POOL & Pool of biomass produced from photosynthesis & $\mathrm{g} \cdot \mathrm{m}^{-2}$ \\
\hline & REPTIL & Number of reproductive tillers (shoots) & $\mathrm{Ntil} \cdot \mathrm{m}^{-2}$ \\
\hline & ROOTB & Root biomass & $\mathrm{g} \cdot \mathrm{m}^{-2}$ \\
\hline & STEMB & Stem biomass & $\mathrm{g} \cdot \mathrm{m}^{-2}$ \\
\hline & STEMP & Sum of temperature above threshold & ${ }^{\circ} \mathrm{C} \cdot$ day \\
\hline & STORB & Storage organ biomass & $\mathrm{g} \cdot \mathrm{m}^{-2}$ \\
\hline & VTIL & Number of vegetative tillers (shoots) & $\mathrm{Ntil} \cdot \mathrm{m}^{-2}$ \\
\hline \multirow[t]{13}{*}{ Rates } & DTEMP & Rate of increase in sum of temperature & ${ }^{\circ} \mathrm{C}$ \\
\hline & PARTL & $\begin{array}{l}\text { Rate of partitioning of assimilates towards } \\
\text { leaves }\end{array}$ & $\mathrm{g} \cdot \mathrm{m}^{-2} \cdot \mathrm{day}^{-1}$ \\
\hline & PARTR & Rate of partitioning of assimilates towards roots & $\mathrm{g} \cdot \mathrm{m}^{-2} \cdot \mathrm{day}^{-1}$ \\
\hline & PARTSO & $\begin{array}{l}\text { Rate of partitioning of assimilates towards } \\
\text { storage organs }\end{array}$ & $\mathrm{g} \cdot \mathrm{m}^{-2} \cdot \mathrm{day}^{-1}$ \\
\hline & PARTS & Rate of partitioning of assimilates towards stems & $\mathrm{g} \cdot \mathrm{m}^{-2} \cdot \mathrm{day}^{-1}$ \\
\hline & RMAT & Rate of tiller (shoot) maturity & $\mathrm{Ntil} \cdot \mathrm{m}^{-2} \cdot \mathrm{day}^{-1}$ \\
\hline & RG & Rate of crop growth & $\mathrm{g} \cdot \mathrm{m}^{-2} \cdot \mathrm{day}^{-1}$ \\
\hline & RMORTV & Rate of mortality of vegetative tillers (shoots) & $\mathrm{Ntil} \cdot \mathrm{m}^{-2} \cdot \mathrm{day}^{-1}$ \\
\hline & RMORTR & Rate of mortality of reproductive tillers (shoots) & $\mathrm{Ntil} \cdot \mathrm{m}^{-2} \cdot \mathrm{day}^{-1}$ \\
\hline & RSENL & Rate of leaf senescence & $\mathrm{g} \cdot \mathrm{m}^{-2} \cdot \mathrm{day}^{-1}$ \\
\hline & RTIL & Rate of tillering (of shoot emergence) & $\mathrm{Ntil} \cdot \mathrm{m}^{-2} \cdot \mathrm{day}^{-1}$ \\
\hline & RTRANSLOC & $\begin{array}{l}\text { Rate of translocation of carbohydrates from } \\
\text { stems to storage organs }\end{array}$ & $\mathrm{g} \cdot \mathrm{m}^{-2} \cdot \mathrm{day}^{-1}$ \\
\hline & LAI & Leaf area index & $\mathrm{m}^{2} \cdot \mathrm{m}^{-2}$ \\
\hline \multirow{2}{*}{$\begin{array}{l}\text { Computed } \\
\text { variables }\end{array}$} & TOTIL & Total number of tillers & $\mathrm{Ntil} \cdot \mathrm{m}^{-2}$ \\
\hline & $\mathrm{k}$ & Coefficient of light extinction & - \\
\hline Parameters & FST & Fraction of sterile tillers (shoots) after flowering & - \\
\hline
\end{tabular}




\begin{tabular}{|c|c|c|c|}
\hline & MAXTIL & Maximum number of tillers (shoots) & $\mathrm{Ntil} \cdot \mathrm{m}^{-2}$ \\
\hline & RRMAT & Relative rate of tiller maturity & Ntil·Ntil ${ }^{-1}$ \\
\hline & STW & Dry biomass of a new tiller (shoot) & $\mathrm{g} \cdot \mathrm{Ntil}^{-1}$ \\
\hline & TBASE & Temperature threshold for crop development & ${ }^{\circ} \mathrm{C}$ \\
\hline & TFLOW & $\begin{array}{l}\text { Sum of temperature above threshold to reach } \\
\text { flowering stage }\end{array}$ & ${ }^{\circ} \mathrm{C} \cdot$ day \\
\hline & TMAT & $\begin{array}{l}\text { Sum of temperature above threshold to reach } \\
\text { crop maturity }\end{array}$ & ${ }^{\circ} \mathrm{C} \cdot$ day \\
\hline & RAD & Daily global radiation & $\mathrm{MJ} \cdot \mathrm{m}^{-2} \cdot \mathrm{day}^{-1}$ \\
\hline \multirow{3}{*}{$\begin{array}{l}\text { Driving functions } \\
\text { Weather }\end{array}$} & TMIN & Daily minimum temperature & ${ }^{\circ} \mathrm{C}$ \\
\hline & TMAX & Daily maximum temperature & ${ }^{\circ} \mathrm{C}$ \\
\hline & CPL & $\begin{array}{l}\text { Coefficient of partitioning of assimilates towards } \\
\text { leaves }\end{array}$ & - \\
\hline \multirow[t]{8}{*}{$\begin{array}{l}\text { Interpolated } \\
\text { variables }\end{array}$} & CPR & $\begin{array}{l}\text { Coefficient of partitioning of assimilates towards } \\
\text { roots }\end{array}$ & - \\
\hline & CPSO & $\begin{array}{l}\text { Coefficient of partitioning of assimilates towards } \\
\text { storage organs }\end{array}$ & - \\
\hline & CPS & $\begin{array}{l}\text { Coefficient of partitioning of assimilates towards } \\
\text { stems }\end{array}$ & - \\
\hline & DVE & $\begin{array}{l}\text { Fraction of assimilates allocated to the } \\
\text { production of new tillers (shoots) }\end{array}$ & - \\
\hline & DVS & Development Stage & - \\
\hline & RRSENL & Relative rate of leaf senescence & $g \cdot g^{-1}$ \\
\hline & RUE & Radiation Use efficiency & $\mathrm{g} \cdot \mathrm{MJ}^{-1}$ \\
\hline & SLA & Specific Leaf Area & $\mathrm{m}^{2} \cdot \mathrm{g}^{-1}$ \\
\hline
\end{tabular}

\section{Crop development}

Unlike growth, which is a continuous process of accumulation, crop development, or crop phenology, is the progress of a given crop trough successive discrete stages over a crop cycle. Two major stages can be distinguished in general: the vegetative and the reproductive stages. Crop development is critical when modeling crop growth, because it determines many physiological processes and parameters directly. For example, the partitioning of assimilates towards the different organs directly depends on the crop development stage. 
Following Penning de Vries et al. (1989), the representation of crop development can be made in a very simple way, by using a quantitative scale from 0 (emergence) to 1 (flowering) and 2 (maturity).

Crop development mainly depends on temperature, and the development stage DVS of a given crop can be computed as:

$$
D_{\text {VVS }}=\text { STEMP }_{t} / \text { TFLOW } \quad \text { if } \text { STEMP }_{t}<T F L O W
$$

and

$$
D V S_{t}=1+\left[\left(S T E M P_{t}-T F L O W\right) /(T M A T-T F L O W)\right] \quad \text { if } S T E M P_{t} \geq T F L O W
$$

Where $S T E M P_{t}$ is the sum of temperature above the crop-specific threshold temperature (TBASE), $T F L O W$ is the sum of temperature required to reach the flowering stage, and TMAT is the sum of temperature required to reach maturity. The schematic relationship between sum of temperature and development stage is given in Fig. 7.3.

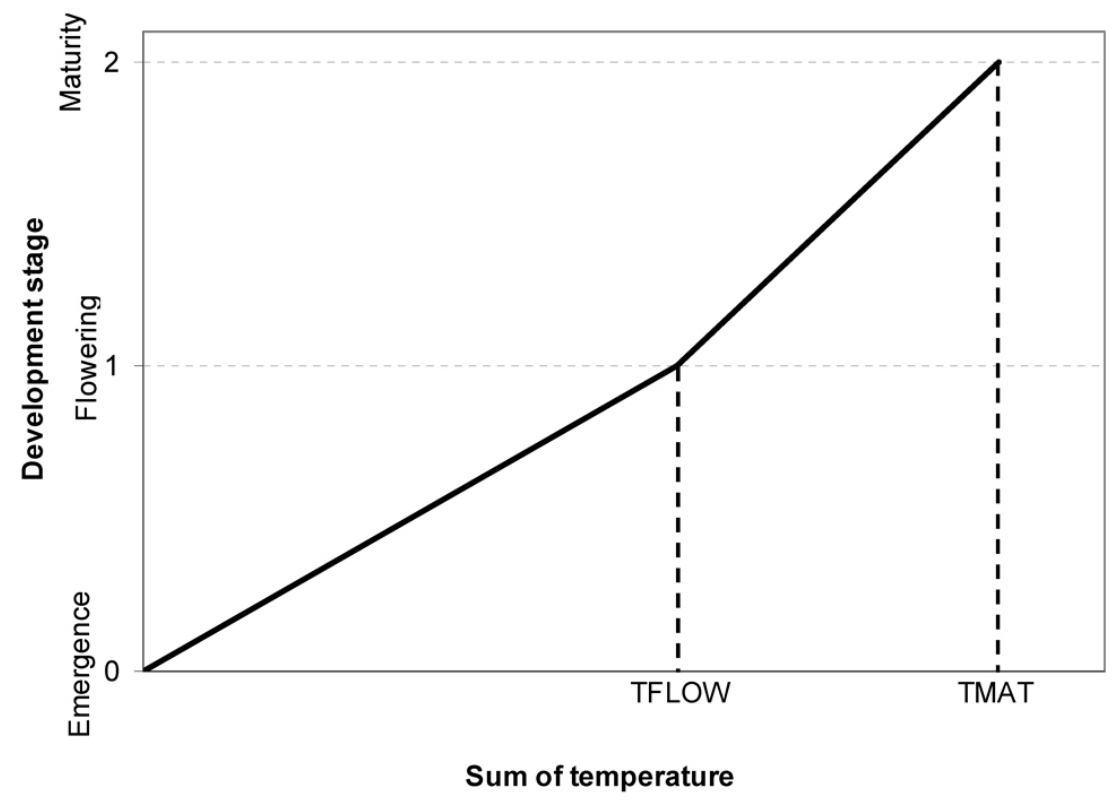

Figure 7.3. Schematic relationship between sum of temperature and development stage.

TFLOW: sum of temperature needed to reach the flowering development stage; TMAT: sum of temperature needed to reach the maturity development stage. The development stage scale is defined as $0=$ emergence, $1=$ flowering, and $2=$ maturity 


\section{Photosynthesis}

Photosynthesis allows the production of assimilates that are made available for plant growth, using radiation energy. The computation of the rate of growth $(R G)$ according to photosynthesis can be done in a synthetic way (e.g., Van Keulen et al., 1982; Sinclair and Muchow, 1999) as:

$$
R G_{t}=R U E_{t} \times R A D_{t} \times\left(1-e^{-k \times L A I_{t}}\right)
$$

where $R A D$ is the daily global solar radiation; $R U E$ is the radiation use efficiency (Monteith, 1977; Sinclair and Muchow, 1999), that is, the amount of assimilates produced per quantity of radiation intercepted by the canopy. The term $[1-\exp (-k \times L A I)]$ is the proportion of light intercepted by the crop, following Beer's law; and $k$ is the coefficient of light extinction. Note that equation (7.5) reflects the same processes embedded in the framework developed by Monteith (equation 7.2).

Crop growth models developed to address crop physiological processes at a finer level of detail have incorporated respiration and transpiration (to, e.g., analyze the effects of water stress or of pests affecting transpiration; e.g., Penning de Vries et al., 1989; Goudriaan and van Laar, 1994). Here, the approach using $R U E$ is used, because it allows capturing and analyzing in a synthetic way a number of physiological processes in crop growth.

$L A I$ can be computed from the dry biomass of leaves $(L E A F B)$ :

$$
L A I_{t}=S L A_{t} \times L E A F B_{t}
$$

where $S L A$ is the specific leaf area (i.e., the leaf area per unit of leaf dry biomass) and is a function of the crop development stage. Young leaves are in general thinner, and thus have a higher SLA than older leaves. It is therefore expected that SLA declines over time.

Radiation use efficiency, $R U E$, represents the overall efficiency of the crop to convert plant biomass from intercepted light. $R U E$ thus encapsulates the efficiency of several processes: gross photosynthesis, respiration, transportation of photosynthates before on-site biosynthesis, and synthesis of complex molecules from photosynthates (e.g., proteins, lipids, polysaccharides). RUE varies depending on (1) the efficiency of photosynthesis, which depends on the concentration of leaf $\mathrm{N}$ and on water availability (Penning de Vries et al., 1989), and; (2) the respective proportion of the different types of organic components synthesized from photosynthates: the energy required for the biosynthesis of a given compound depends on the type of organic group it belongs to (Penning de Vries et al., 1989). For example, lipids require much more energy (that is, more glucose) to be synthesized than 
carbohydrates, the proteins being in an intermediate position (Penning de Vries et al., 1989). The proportion of compounds synthesized depends on the crop development stage.

Thus, $R U E$ varies depending on the crop species, on the crop development stage, and on nutrients and water supply of a crop (Sinclair and Muchow, 1999).

The amount of assimilates that are made available for plant growth $(P O O L)$ is accumulated daily at the rate of growth, $R G$ :

$$
\mathrm{POOL}_{t+\Delta t}=\mathrm{POOL}_{t}+\left(R G_{t} \times \Delta t\right)
$$

\section{Partitioning of assimilates}

Assimilates that are accumulated daily are partitioned to the different plant organs. Most crop plants develop four broad types of organs: leaves, stems, roots, and storage organs (e.g., grains, tubers). The increase in dry biomass of the different crop organs can be computed as follows:

$$
\begin{gathered}
\text { LEAFB }_{t+\Delta t}=\text { LEAFB }_{t}+\left(\text { PARTL }_{t} \times \Delta t\right) \\
\text { STEMB }_{t+\Delta t}=\text { STEMB }_{t}+\left(\text { PARTS }_{t} \times \Delta t\right) \\
\text { ROOTB }_{t+\Delta t}=\text { ROOTB }_{t}+\left(\text { PARTR }_{t} \times \Delta t\right) \\
\text { STORB }_{t+\Delta t}=\text { STORB }_{t}+\left(\text { PARTSO }_{t} \times \Delta t\right)
\end{gathered}
$$

where PARTL, PARTS, PARTR, and PARTSO are the daily flows of assimilates towards leaves, stems, roots, and storage organs, respectively. These flows depend on coefficients of partitioning, which in turn depend on the development stage:

$$
\begin{aligned}
& \text { PARTL }_{t}=\mathrm{POOL}_{t} \times C P L_{t} \times\left(1-C P R_{t}\right) \\
& \text { PARTS }_{t}=P O O L_{t} \times C P S_{t} \times\left(1-C P R_{t}\right) \\
& \text { PARTR }_{t}=\mathrm{POOL}_{t} \times C P R_{t} \\
& \text { PARTSO }_{t}=\mathrm{POOL}_{t} \times C P S O_{t} \times\left(1-C P R_{t}\right)
\end{aligned}
$$

where $C P L, C P S, C P R$, and $C P S O$ are the partitioning coefficients of assimilates to leaves, stems, roots, and storage organs, respectively, at the development stage at date $t$. CPL, CPST, and CPSO represent partitioning coefficients relative to the biomass partitioned above ground. $C P R$ represents the coefficient of partitioning towards roots, relative to the total plant biomass. 
Assimilates produced by photosynthesis are therefore partitioned towards the plant organs, and equation (7.7) becomes:

$$
P O O L_{t+\Delta t}=P O O L_{t}+\left[\left(R G_{t}-P A R T L_{t}-P A R T S_{t}-P A R T R_{t}-P A R T S O_{t}\right) \times \Delta t\right]
$$

In general, partitioning towards roots, stems, and leaves occurs until flowering. From this stage onwards, most, if not all, assimilates are partitioned towards the storage organs (Fig. 7.4).

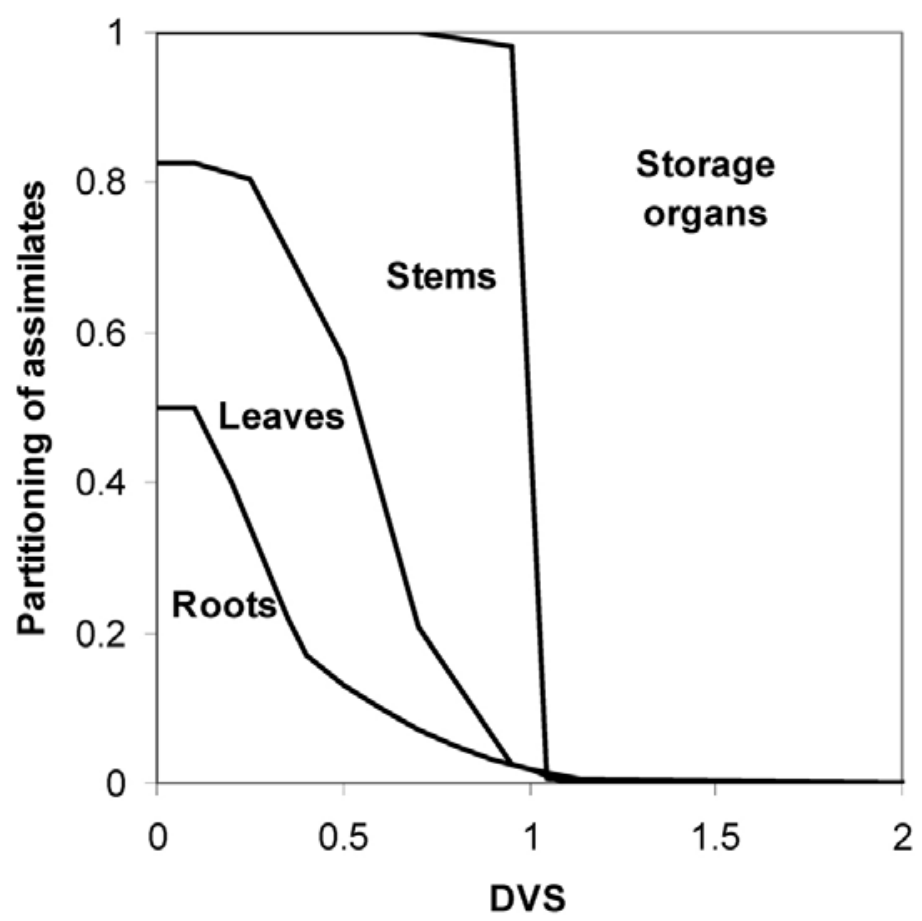

Figure 7.4. Typical dynamics of partitioning of assimilates towards the different organs in the case of a cereal.

\section{Leaf senescence}

Leaf senescence refers to physiological ageing and occurs towards the end of the crop cycle.

Leaf senescence can be represented by a loss of leaf dry biomass (RSENL), which can be made proportional to a relative rate of leaf senescence $(R R S E N L)$ and to the dry biomass of leaves (LEAFB), with RRSENL depending on the development stage.

$$
R S E N L_{t}=R R S E N L_{t} \times L E A F B_{t}
$$


Thus equation (7.8) becomes:

$$
L E A F B_{t+\Delta t}=L E A F B_{t}+\left(\left(P A R T L_{t}-R S E N L_{t}\right) \times \Delta t\right)
$$

\section{Accumulation and redistribution of reserves}

Carbohydrates can be stored in stems or roots before being translocated towards storage organs (Penning de Vries et al., 1989). In the case of cereals, this can be translated in a simple way by simulating a flow of biomass from the stems towards the storage organs after flowering. Equations (7.9) and (7.11) thus become:

$$
\begin{aligned}
& S T E M B_{t+\Delta t}=S_{T E M B}+\left(\left(P_{t} A R T S T_{t}-R_{R A R S L O C}\right) \times \Delta t\right) \\
& \left.S_{\text {TORB }}+\Delta t=\text { STORB }_{t}+\left(\left(\text { PARTSO }_{t}+\text { RTRANSLOC }_{t}\right) \times \Delta t\right)\right)
\end{aligned}
$$

where RTRANSLOC $C_{t}$ is the daily rate of translocation of starch from stems to storage organs.

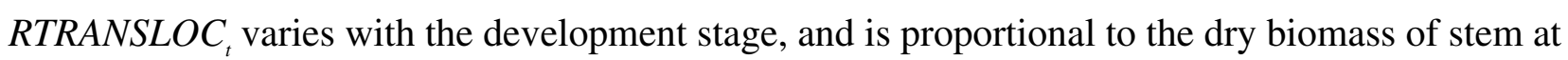
flowering.

\section{Dynamics of tillers (or shoots)}

In the following, we shall refer to tillers or plant shoots in an equivalent manner, as GENECROP can be used equivalently for a cereal (where the aerial part of a plant - the unit of a crop stand - consists of tillers as sub-units) or a dicotyledon (where shoots could be considered instead as sub-units) crop. Crop growth simulation models do not usually consider the dynamics of plant subunits, since consideration of the functioning of a system's unit is sufficient to understand the behavior of the system at the higher level of integration (Penning de Vries and Van Laar, 1982). When aiming at simulating the effects of pest injuries on crop growth, however, the simulation of tiller (or shoot) dynamics may allow direct and relevant simulation of the way injuries affect tiller (shoot) mortality. The tiller (shoot) dynamic can be modeled by considering first vegetative tillers (shoots), which multiply during the tillering (shoot emission) phase. The tillering rate is assumed to be proportional to the rates of leaf and stem growth:

$$
R T I L_{t}=\left(P A R T L_{t}+P A R T S_{t}\right) \times S T W
$$


where RTIL is the tillering rate; PARTL is the rate of leaf growth; PARTS is the rate of stem growth; $S T W$ is the dry biomass of one new tiller.

During the tillering phase, leaf and stem growth contribute progressively less to generating new tillers, and more to leaf production, leaf expansion, and stem elongation. Tiller production is therefore seen in the model to compete with tiller growth, with respect to assimilate allocation to stems and leaves. This is reflected by introducing the factor (1-(VTIL/MAXTIL)) in equation (7.21), where VTIL and MAXTIL represent the number of vegetative tillers and the maximum number of tillers, respectively. Tillering is furthermore governed by crop development: when the crop reaches the maximum tillering stage, assimilates are not allocated for tillering any more. This is reflected by a multiplicative term, DVE, which is made dependent on development stage. Equation (7.21) thus becomes:

$$
R T I L_{t}=\left(P A R T L_{t}+P A R T S_{t}\right) \times S T W \times\left[1-\left(V T I L_{t} / M A X T I L\right)\right] \times D V E_{t}
$$

The shift from the vegetative phase to the reproductive phase corresponds to the maturation of vegetative tillers (shoots), which become reproductive. This is reflected by a rate of maturity (RMAT; which depends on development stage), which flows from the number of vegetative tillers (VTIL) to the number of reproductive tillers $(R E P T I L)$ :

$$
R M A T_{t}=R R M A T \times V T I L_{t}
$$

where RRMAT is the relative rate of tiller maturity.

A fraction of the vegetative tillers, FST, may remain vegetative and not produce any storage organ. Furthermore, between maximum tillering and flowering stages, some of the younger tillers die, due to competition for light and nutrients with the other tillers. The dynamics of vegetative tillers and of reproductive tillers is described by equations (7.24) and (7.25), respectively:

$$
\begin{aligned}
& V T I L_{t+\Delta t}=V T I L_{t}+\left(R T I L_{t}-R_{M O R T V}-R M A T_{t}\right) \times \Delta t \\
& \operatorname{REPTIL}_{t+\Delta t}=R_{t} P T I L_{t}+\left(R M A T_{t}-R M O R T R_{t}\right) \times \Delta t
\end{aligned}
$$

with:

$$
\begin{aligned}
& R M O R T V_{t}=R_{R M O R T_{t}} \times V T I L_{t} \\
& \text { RMORTR }_{t}=R_{R M O R T_{t}} \times R \text { RPTIL }_{t}
\end{aligned}
$$


where $R R M O R T_{t}$ is the relative rate of tiller mortality, and depends on development stage.

\section{Model parameters}

The simulation starts at 15 days after crop establishment (DACE), and ends when DVS reaches 2. Model inputs and parameters have been chosen to be within the range of values found for crops (or cereals) under favorable environments.

Weather variables used as inputs (driving functions) are daily minimum and maximum temperature (which drive the development stage) and daily radiation (which drives the rate of crop growth). Minimum and maximum temperature have been set to $24^{\circ} \mathrm{C}$ and $30^{\circ} \mathrm{C}$, respectively, and are kept constant over the duration of the simulation for the sake of simplicity. Global radiation is also constant over time, and has been set to $17 \mathrm{~mJ} \cdot \mathrm{m}^{-2} \cdot$ day $^{-1}$.

Parameters for crop development have been set to $1500{ }^{\circ} \mathrm{C}$.day and $2000{ }^{\circ} \mathrm{C} \cdot$ day for $T F L O W$ and TMAT, respectively, and to $8^{\circ} \mathrm{C}$ for TBASE (threshold temperature under which the crop does not develop). The main parameters for crop growth have been set to the following values:

$R U E=1.2 \mathrm{~g} \cdot \mathrm{MJ}^{-1}$ (value for a crop under favorable conditions; Monteith, 1977; Sinclair and Muchow, 1999)

$k=0.6$ (value for a canopy with erect leaves; Goudriaan, 1977) SLA decreases from 0.037 to $0.018 \mathrm{~m}^{2} \cdot \mathrm{g}^{-1}$ from emergence (DVS=0) to flowering (DVS=1), and from 0.018 to $0.017 \mathrm{~m}^{2} \cdot \mathrm{g}^{-1}$ from flowering to maturity (derived from Willocquet et al., 2004).

Coefficients of partitioning towards the different organs according to the development stage are derived from Willocquet et al. (2004). The maximum number of tillers has been set to 900 tillers $\cdot \mathrm{m}^{-2}$.

\section{Simulations}

The STELLA model GENECROP.STMX will allow you to:

- explore the model structure and equations,

- explore the model inputs,

- explore the model outputs, and

- run the model with varying values of $R U E$, so as to observe the effects these changes have on the dynamics of the crop growth and on final yield.

The simulated crop growth using GENECROP is displayed in Fig. 7.5. 


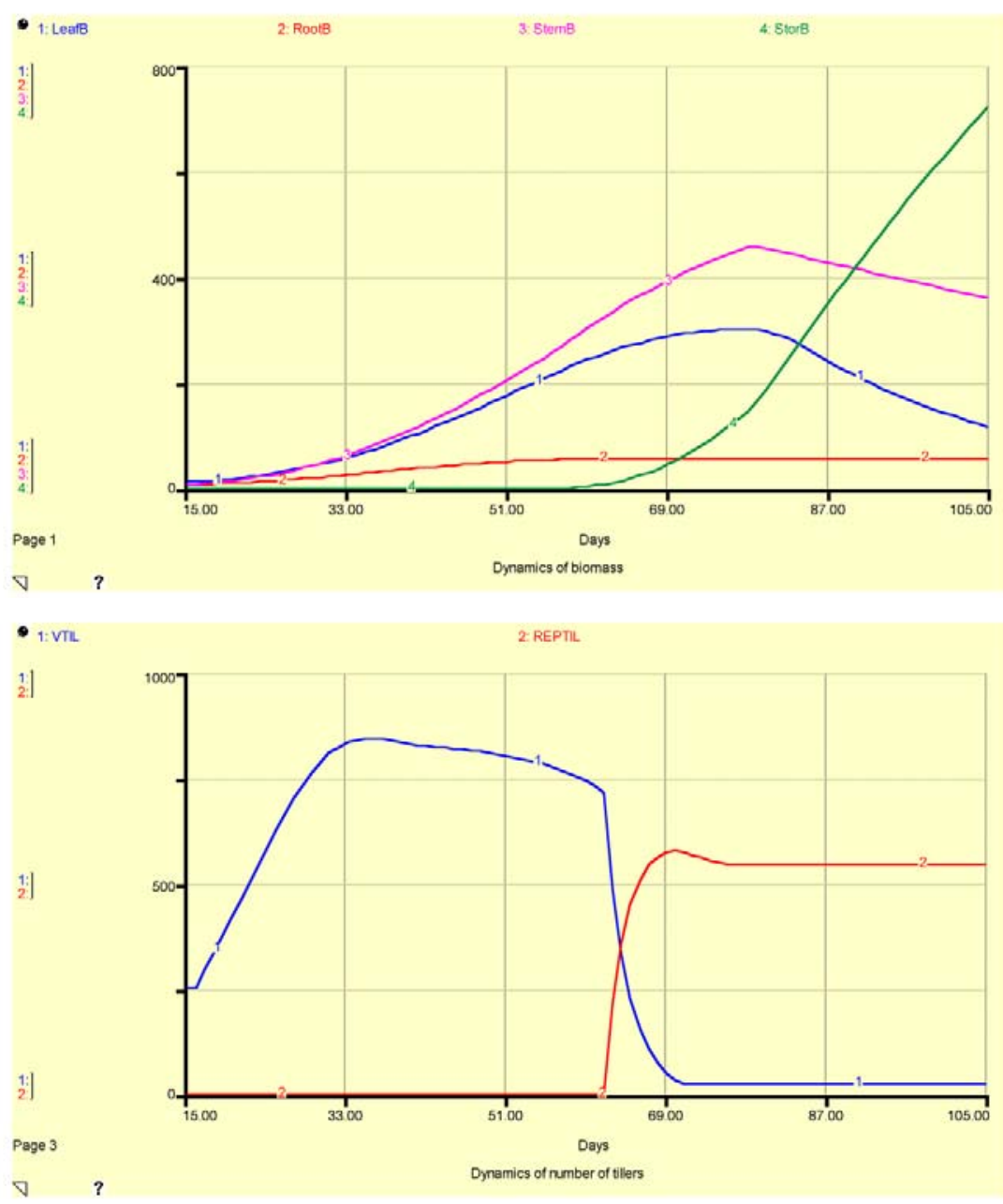

Figure 7.5. Simulated outputs of biomass (upper graph) and number of tillers (lower graph) using GENECROP

The model outputs reflect the hypotheses captured in the different equations of the model. Crop reaches maturity when DVS equals 2, that is, at 105 days after crop establishment (DACE). The biomass of roots increases regularly until 51 DACE, then tapers off at around 60 DACE. Leaf biomass increases according to a sigmoid-like shape until flowering (which occurs at 77 DACE), and then declines as leaf senescence takes place. Stem biomass increases regularly until flowering, and then declines nearly linearly as carbohydrates are translocated towards the storage organs. The dry biomass of storage organs increases exponentially, and then nearly linearly, when all assimilates are partitioned towards these organs towards the end of the crop cycle. The final yield is about $700 \mathrm{~g} \cdot \mathrm{m}^{-2}$, that is, 7 $\mathrm{t} \cdot \mathrm{ha}^{-1}$.

The number of vegetative tillers increases until maximum tillering, then decreases because of tiller mortality due to competition, and finally decreases because vegetative tillers become reproductive. The number of reproductive tillers increases when vegetative tillers reach the 
reproductive stage, and then remains at the same level until the end of the crop cycle, about 500 tillers $\cdot \mathrm{m}^{-2}$.

\section{Concluding remarks}

The reader will have noticed that this chapter deals with annual crops, with a definite tinge towards cereals. This is a reflection of the authors' main interest, but mainly because such systems are comparatively simple. Complication (but not necessarily complexity) may emerge when:

- one considers crops whose lifespan is long and/or covers several seasons, such as cassava, sugarcane, alfalfa, pyrethrum, or banana;

- the focus of research concerns perennials, e.g., fruit trees, grapevine, or blueberries;

- crop species or genotypes with indeterminate growth are considered, such as tomatoes or beans.

Many of the ideas that have been forwarded in this chapter are relevant to such crops, however. One, in particular, is the remobilization of carbohydrates from one season to the other, which, for example, explains the yearly oscillations of coffee yield in a plantation, the associated variation of coffee susceptibility to rust, and so, the yearly oscillations of coffee rust epidemics (Avelino et al., 2004).

\section{Summary}

This chapter describes:

- The RI-RUE concept.

- The main processes involved in crop growth.

- How they are captured in a quantitative and dynamic way into a generic simulation model, GENECROP.

- The equations, parameters, and flowchart of GENECROP.

- Includes the STELLA file, which can be used to explore the model structure and the effect of some parameters on the simulated dynamics of the model variables.

\section{References}

Avelino, J., Willocquet, L., and Savary, S. 2004. Effects of crop management patterns on coffee rust epidemics. Plant pathology 53: 541-547.

Goudriaan, J. 1977. Crop Micrometeorology: a Simulation study. Simulation Monographs. Pudoc, Wageningen. 
Goudriaan, J., and van Laar, H. H. 1994. Modelling Potential Crop Growth Processes. Kluwer Academic Publishers, Dordrecht.

Johnson K. B., Johnson S. B., and Teng, P. S. 1986. Development of a simple potato growth model for use in crop-pest management. Agric. Sys. 19:189-209.

Kiniry, J. R., Bean, B., Xie, Y., and Chen, P. 2004. Maize yield potential: critical processes and simulation modeling in a high-yielding environment. Agric. Sys. 82:45-56.

Monsi, M., and Saeki, T. 1953. Uber den Lichtfaktor in den Pflanzengesellschaften und sein Bedeutung für die Stoffproduktion. Jpn. J. Bot. 14:22-52.

Monteith, J. L. 1977. Climate and the efficiency of crop production in Britain. Phil. Trans. Roy. Soc. Lond. 281:277-294.

Penning de Vries, F. W. T., Jansen, D. M., ten Berge, H. F. M., and Bakema, A., eds. 1989. Simulation of Ecophysiological Processes of Growth in Several Annual Crops. IRRI, Los Baños, and Pudoc, Wageningen.

Penning de Vries, F. W. T., and Van Laar, H. H. 1982. Simulation of Plant Growth and Crop Production. Pudoc, Wageningen.

Richter, G. M., Haggard, K. W., and Mitchell, R. A. C. 2001. Modelling radiation interception and radiation use efficiency for sugar beet under variable climatic stress. Agric. Forest Meteorol. 109:13-25.

Sinclair, T. R. 1986. Water and nitrogen limitations in soybean grain production. I. Model development. Field Crops Res. 15:125-141.

Sinclair, T. R., and Muchow, R. C. 1999. Radiation-use efficiency. Adv. Agron. 65:215-265.

Steer, B. T., Milroy, S. P., and Kamona R. M. 1993. A model to simulate the development, growth and yield of irrigated sunflower. Field Crops Res. 32:83-99.

Thornley, J. H. M., and France, J. 2007. Mathematical Models in Agriculture. Quantitative Methods for the Plant, Animal and Ecological Sciences, 2nd ed. CABI, Wallingford.

Van Keulen, H., Penning de Vries, F. W. T., and Drees, E. M. 1982. A summary model for crop growth. Pages 87-97 in: Simulation of Plant Growth and Crop Production. F. W. T. Penning de Vries and H. H. van Laar, eds. Pudoc, Wageningen.

Whisler, F. D., Acock, B., Baker, D. N., Fye, R. E., Hodges, H. F., Lambert, J. R., Lemmon, H. E., McKinion, J. M., and Reddy, V. R. 1986. Crop simulation models in agronomic systems. Adv. Agron. 40:141-208.

Willocquet, L., Elazegui, F. A., Castilla, N., Fernandez, L., Fischer, K. S., Peng, S., Teng, P. S., Srivastava, R. K., Singh, H. M., Zhu, D., and Savary, S. 2004. Research priorities for rice pest 
management in tropical Asia: a simulation analysis of yield losses and management efficiencies. Phytopathology 94:672-682.

Yin, X., Struik, P. C., and Kropff, M. J. 2004. Role of crop physiology in predicting gene-to-phenotype relationships. Tr. Plant Sci. 9:427-432.

\section{Suggested reading}

Charles-Edwards, D. A., Doley, D., and Rimmington, G. M. 1986. Modelling Plant Growth and Development. Academic Press, Sydney.

Sinclair, T. R., and Seligman, N. G. 1996. Crop modeling: from infancy to maturity. Agron. J. 88: 698704.

van Ittersum, M. K., Leffelaar, P. A., van Keulen, H., Kropff, M. J. Bastiaans, L., and Goudriaan, J. 2003. On approaches and applications of the Wageningen crop models. Eur. J. Agron. 18:201-234.

Exercises and questions

1. What is the difference between (crop) growth and development?

2. What is the dimension of a rate of crop growth? What is the dimension of a rate of crop development?

3. The increase in crop biomass directly depends on several factors, including
a. leaf biomass
b. radiation
c. LAI
d. temperature

4. Radiation Use Efficiency, RUE can be expressed with the unit(s)
a. $\left[\mathrm{g} \cdot \mathrm{MJ}^{-1} \cdot \mathrm{m}^{-2}\right]$
b. $\left[\mathrm{g} \cdot \mathrm{MJ}^{-1}\right]$
c. $\left[\mathrm{MJ} \cdot \mathrm{g}^{-1} \cdot \mathrm{m}^{-2}\right]$
d. $\left[\right.$ g. $\mathrm{MJ}^{-1} \cdot \mathrm{m}^{-2} \cdot$ day-1]

5. Crop development

a. represents changes in crop biomass 

b. mainly depends on temperature
c. mainly depends on radiation
d. determines the partitioning of assimilates towards plant organs

Answers to exercises and questions

1. Crop growth is the accumulation (and possibly decrease) of biomass over time; whereas crop development represents the passing of a crop (seen as a cohort, i.e., a population of plants which have a similar development stage at a given point of time) through the successive development stages of its life cycle. For instance, in cereals, development spans from seeds and their germination, to ripening of ears.

2. The rate of crop growth is measured as a quantity of biomass $[\mathrm{M}]$ per unit time [T], so a rate of crop growth is measured as: [M.T ${ }^{-1}$. Development is, by essence, a qualitative attribute, and so does not have dimension [ - ], so a rate of development is $\left[\mathrm{T}^{-1}\right]$.

3. b: radiation, and c: LAI.

4. b: $\left[\mathrm{g} \cdot \mathrm{MJ}^{-1}\right]$.

5. b: mainly depends on temperature, and d: determines the partitioning of assimilates towards plant organs. 
Appendix 7.1. Program listing of GENECROP

$\operatorname{LeafB}(\mathrm{t})=\operatorname{LeafB}(\mathrm{t}-\mathrm{dt})+($ PartL $-\mathrm{RSenL}) * \mathrm{dt}$

INIT LeafB $=10$

INFLOWS:

PartL $=$ CPL $*$ Pool

OUTFLOWS:

RSenL $=$ rrsen $*$ LeafB

$\operatorname{MaxStemb}(\mathrm{t})=\operatorname{MaxStemb}(\mathrm{t}-\mathrm{dt})+(\operatorname{rmaxstemb}) * \mathrm{dt}$

INIT MaxStemb $=6$

INFLOWS:

rmaxstemb $=$ PartLS

$\operatorname{Pool}(\mathrm{t})=\operatorname{Pool}(\mathrm{t}-\mathrm{dt})+($ RGrowth - PartS - PartL - PartSO - PartR $) * d t$

INIT Pool $=0$

INFLOWS:

RGrowth $=$ RAD $*$ RUE* $\left(1-E X P\left(-k^{*} L A I\right)\right)$

OUTFLOWS:

PartS $=$ CPS $*$ Pool

PartL $=$ CPL $*$ Pool

PartSO $=$ CPP $*$ Pool

PartR $=$ CPR $*$ Pool

$\operatorname{REPTIL}(\mathrm{t})=\operatorname{REPTIL}(\mathrm{t}-\mathrm{dt})+($ Rmat - Rmortr $) * \mathrm{dt}$

INIT REPTIL $=0$

INFLOWS:

Rmat $=$ if DVS $<0.8$ or DVS $>1$ then 0 else if VTIL $<$ FST $*$ Totil then 0 else RRMAT $*$ VTIL

OUTFLOWS:

Rmortr $=$ rrmort $*$ REPTIL

$\operatorname{RootB}(\mathrm{t})=\operatorname{RootB}(\mathrm{t}-\mathrm{dt})+(\operatorname{PartR}) * \mathrm{dt}$

INIT RootB $=5$

INFLOWS:

PartR $=$ CPR $*$ Pool

$\operatorname{StemB}(\mathrm{t})=\operatorname{StemB}(\mathrm{t}-\mathrm{dt})+($ PartS - RTransloc $) * \mathrm{dt}$

INIT StemB $=6$

INFLOWS: 
PartS $=$ CPS $*$ Pool

OUTFLOWS:

RTransloc $=\operatorname{IF}($ DVS $>1)$ then ddist else 0

$\operatorname{STEMP}(\mathrm{t})=\operatorname{STEMP}(\mathrm{t}-\mathrm{dt})+($ Dtemp $) * d t$

INIT STEMP $=320$

INFLOWS:

Dtemp $=(($ TMAX + TMIN $) / 2)-$ TBASE

$\operatorname{StorB}(\mathrm{t})=\operatorname{StorB}(\mathrm{t}-\mathrm{dt})+(\operatorname{PartSO}+\mathrm{RTransloc}) * \mathrm{dt}$

INIT StorB $=0$

INFLOWS:

PartSO = CPP*Pool

RTransloc $=\operatorname{IF}($ DVS $>1)$ then ddist else 0

$\operatorname{VTIL}(\mathrm{t})=\operatorname{VTIL}(\mathrm{t}-\mathrm{dt})+($ Rtil - Rmat $-\mathrm{Rmrtv}) * \mathrm{dt}$

INIT VTIL $=250$

INFLOWS:

Rtil $=$ PartLS $*$ STW $*(1-($ VTIL/Maxtil $)) *$ DVE

OUTFLOWS:

Rmat $=$ if DVS $<0.8$ or DVS $>1$ then 0 else if VTIL $<$ FST $*$ Totil then 0 else RRMAT*VTIL

Rmrtv $=($ rrmort $*$ VTIL)

$\mathrm{CPL}=\mathrm{CPPL} *(1-\mathrm{CPR})$

$\mathrm{CPP}=\mathrm{CPPP} *(1-\mathrm{CPR})$

$\mathrm{CPS}=(1-\mathrm{CPL}-\mathrm{CPP}) *(1-\mathrm{CPR})$

ddist $=0.005 *$ MaxStemb

DVS $=$ if stemp<TFLOW then STEMP/TFLOW ELSE 1+((STEMP-TFLOW)/(TMAT-TFLOW $))$

$\mathrm{FST}=0.05$

$\mathrm{k}=0.6$

$\mathrm{LAI}=\mathrm{LeafB} * \mathrm{SLA}$

Maxtil $=900$

PartLS $=$ PartL + PartS

$\mathrm{RAD}=17$

RRMAT $=0.3$

RUE $=1.2$

$\mathrm{STW}=20$

TBASE $=8$ 
TFLOW $=1500$

$\mathrm{TMAT}=2000$

TMAX $=30$

$\mathrm{TMIN}=24$

Totil $=$ VTIL+REPTIL

$\mathrm{CPPL}=\mathrm{GRAPH}(\mathrm{DVS})$

(0.00, 0.55), (0.1, 0.536), (0.2, 0.521), (0.3, 0.507), (0.4, 0.493), (0.5, 0.479), (0.6, 0.464), (0.7, 0.45),

$(0.8,0.3),(0.9,0.15),(1,0.00),(1.10,0.00),(1.20,0.00),(1.30,0.00),(1.40,0.00),(1.50,0.00),(1.60$,

$0.00),(1.70,0.00),(1.80,0.00),(1.90,0.00),(2.00,0.00)$

$\mathrm{CPPP}=\mathrm{GRAPH}(\mathrm{DVS})$

$(0.00,0.00),(0.05,0.00),(0.1,0.00),(0.15,0.00),(0.2,0.00),(0.25,0.00),(0.3,0.00),(0.35,0.00)$, $(0.4,0.00),(0.45,0.00),(0.5,0.00),(0.55,0.00),(0.6,0.00),(0.65,0.00),(0.7,0.00),(0.75,0.00),(0.8$, 0.143), (0.85, 0.286), (0.9, 0.429), (0.95, 0.571), (1.00, 0.714), (1.05, 0.857), (1.10, 1.00), (1.15, 1.00), $(1.20,1.00),(1.25,1.00),(1.30,1.00),(1.35,1.00),(1.40,1.00),(1.45,1.00),(1.50,1.00),(1.55,1.00)$, $(1.60,1.00),(1.65,1.00),(1.70,1.00),(1.75,1.00),(1.80,1.00),(1.85,1.00),(1.90,1.00),(1.95,1.00)$, $(2.00,1.00)$

$\mathrm{CPR}=\mathrm{GRAPH}(\mathrm{DVS})$

(0.00, 0.3), (0.1, 0.263), (0.2, 0.225), (0.3, 0.188), (0.4, 0.15), (0.5, 0.112), (0.6, 0.075), (0.7, 0.038), $(0.8,0.00),(0.9,0.00),(1,0.00),(1.10,0.00),(1.20,0.00),(1.30,0.00),(1.40,0.00),(1.50,0.00)$, $(1.60,0.00),(1.70,0.00),(1.80,0.00),(1.90,0.00),(2.00,0.00)$

\section{$\mathrm{DVE}=\mathrm{GRAPH}(\mathrm{DVS})$}

$(0.00,1.00),(0.4,1.00),(0.8,0.00),(1.20,0.00),(1.60,0.00),(2.00,0.00)$

rrmort $=$ GRAPH(DVS)

(0.00, 0.00), (0.1, 0.00), (0.2, 0.00), (0.3, 0.00), (0.4, 0.00), (0.5, 0.02), (0.6, 0.02), (0.7, 0.02), (0.8, 0.02), (0.9, 0.02), (1, 0.00), (1.10, 0.00), (1.20, 0.00), (1.30, 0.00), (1.40, 0.00), (1.50, 0.00), (1.60, $0.00),(1.70,0.00),(1.80,0.00),(1.90,0.00),(2.00,0.00)$

rrsen $=$ GRAPH $($ DVS $)$

(0.00, 0.00), (0.1, 0.00), (0.2, 0.00), (0.3, 0.00), (0.4, 0.00), (0.5, 0.00), (0.6, 0.00), (0.7, 0.00), (0.8, 0.00), (0.9, 0.00), (1, 0.00), (1.10, 0.013), (1.20, 0.026), (1.30, 0.04), (1.40, 0.04), (1.50, 0.04), (1.60, $0.04),(1.70,0.04),(1.80,0.04),(1.90,0.04),(2.00,0.04)$

$\mathrm{SLA}=\mathrm{GRAPH}(\mathrm{DVS})$

$(0.00,0.037),(1.00,0.018),(2.00,0.017)$ 


\section{Chapter 8. Modeling Yield Losses Due to Pests - The GENEPEST Structure}

The analysis and modeling of crop losses is central to plant protection in general, and to plant pathology, in particular: no plant protection scientific reasoning could possibly exist without a measure of crop losses (Chiarappa, 1971; Rabbinge et al., 1989; Savary et al., 2006; Teng, 1987; Zadoks, 1985; Zadoks and Schein, 1979). In many ways, the applied side of phytopathology, as a science, would thus not exist if crop losses to diseases did not occur. Ironically, the information on crop loss is scarce for a number of reasons we shall not elaborate here. Simulation modeling is one approach to complement the existing data, upscale them, and project ourselves in future environmental, social, and technical scenarios. However, this is only possible if reliable field data are available in sufficient number to assess the outputs of models - simulation outputs cannot be seen per se as substitute for measured realities. This latter point cannot be addressed here despite its critical importance.

This chapter introduces the effects of pests (pathogens, but also animal pests, and weeds) on crop growth and how they can be incorporated into crop growth simulation models in order to model yield losses. Crop losses, or more specifically yield losses, occur because the physiology of the growing crop is negatively affected by pests in a dynamic way over time as crop both grows (i.e., increases in biomass) and develops (i.e., passes through the different stages of its physiological development). As a necessary first step to achieve the modeling of yield losses we therefore need to introduce concepts that are related to yield levels and damage mechanisms because they represent the conceptual basis of modeling yield losses. The effects of pests on crop growth using the so called "radiation interception - radiation use" (RI-RUE) framework discussed in the previous chapter will then be addressed again. Lastly, the implementation of damage mechanisms into a crop growth model will be presented and illustrated.

Developing simulation models that integrate the dynamic effects of damage mechanisms of injuries caused by pests, and their translation into yield reduction can provide several types of outcomes, both scientific and practical. Note that, because we deal with (physiological) damage mechanisms on the growing crop, the focus is not on the pathogens (pests) themselves, but the injuries each pathogen (pest) may cause: one pathogen (pest) may cause one or several (and quite different) injuries.

Such models enable, for instance, one to gain:

- A better understanding of processes involved in the attrition of crop growth and yield caused by pest injuries; this is the heuristic value of (simple) simulation models. In that case, the system's 
behavior is analyzed and allows pinpointing knowledge gaps and deriving hypotheses on the system's functioning.

- A better view of the respective importance of pests, with respect to the yield losses they can cause: simulation of yield losses caused by one injury in isolation, versus a combination of injuries, and their respective contribution to yield loss, allows ranking of individual diseases (pests) according to the yield losses they cause (or might cause under pre-set scenarios).

- A prospective view of yet-to-achieve progress in disease (pest) management. Simulation of yield gained from improved management tools or strategies provides a formal and quantitative basis for strategic decisions in pest management, including setting research priorities. This applies, in particular, but not solely, to plant breeding, where research efforts are both long and expensive. One can also think of policy applications for better natural resource use and conservation, improvement of production situations, or landscape management.

Important note: crop loss models as presented here therefore do not simulate the dynamics of epidemics (or of pests, in general), but the dynamics of yield build-up (with or without injuries). As you will see in this chapter, modeling of damage mechanisms and yield losses entails processes (and therefore involves model structures) that are directly connected to the growing crop. As a result, the emphasis in modeling yield losses presented here is completely different from the standpoint used in addressing the modeling of epidemics (described in Chapters 4, 5 and 6 of this module). The crop loss modeling approach in this chapter is instead a direct application of Chapter 7.

This chapter describes concepts used for yield loss modeling, and illustrates how these concepts can be implemented when developing a simulation model for yield loss. Such an approach has been applied to a number of crop pests, for example in the case of groundnut rust and leaf spots (Savary et al., 1990; Savary and Zadoks, 1992), multiple pests of potato (Johnson, 1992), rice leaf blast (Bastiaans, 1993), virus diseases (Madden et al., 2000), multiple pests of rice (Willocquet et al., 2000) and multiple pests of wheat (Willocquet et al., 2008).

\section{Concepts and definitions related to yield levels, production situations and injuries}

The concept of yield levels (potential, attainable, actual) and the factors which determine them (Chiarappa, 1971; Zadoks and Schein, 1979; Rabbinge et al., 1989; Rabbinge, 1993) provides a framework which has been, and still is, largely used to address the performances of agrosystems from 
the biophysical and socio-economical points of view (van Ittersum and Rabbinge, 1997). Fig. 8.1 provides an overview of yield levels and the factors which determine them.

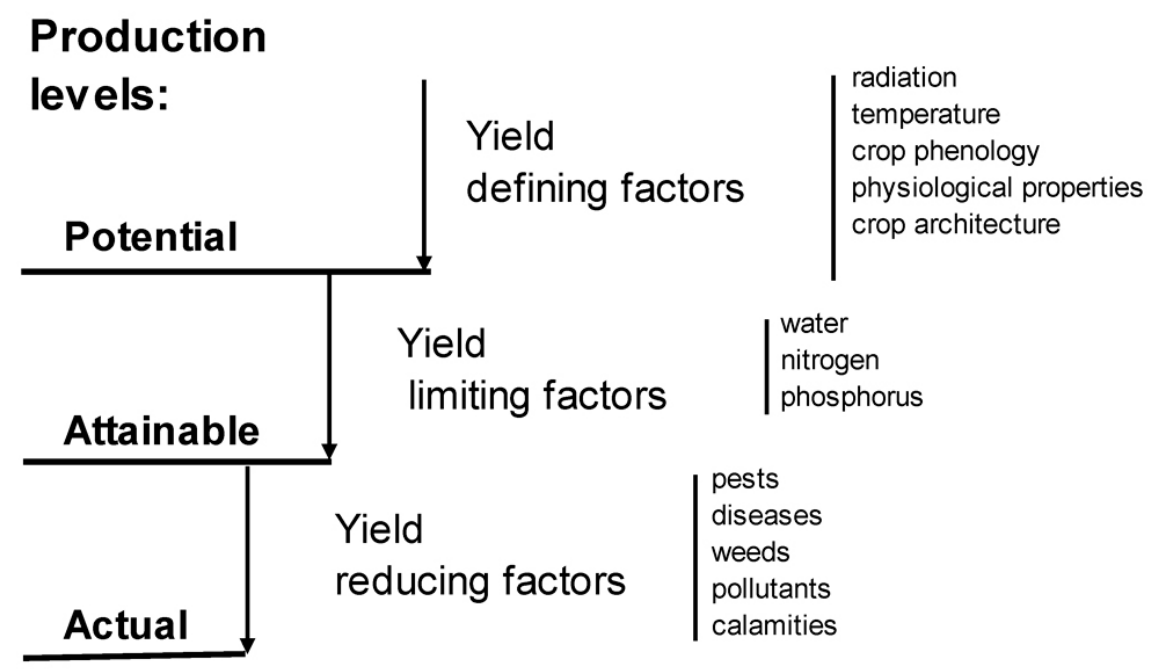

Figure 8.1. Relationships among potential, attainable and actual yields and growth-defining, growth limiting and growth-reducing factors (Rabbinge, 1993; van Ittersum and Rabbinge, 1997).

The potential yield ( $Y p$ ) of a crop is determined by defining factors: radiation, temperature, and morphological and physiological attributes determined by the genotype of the crop. The potential yield thus corresponds to the yield that would be produced by a crop grown under optimum conditions.

The attainable yield $(Y a)$ is determined by the defining factors in combination with limiting factors: water and nutrients. The attainable yield corresponds also to the yield that would be produced by a crop when free of injuries.

The combination of yield defining and yield limiting factors can be embedded in the concept of production situation (de Wit and Penning de Vries, 1982; Savary and Zadoks, 1992; Rabbinge et al., 1993). The attainable yield of a given crop thus corresponds to the production situation under which this crop is grown.

The attainable yield can be reduced by the effect of reducing factors such as pest (disease, insects, weeds) injuries. An injury is a visible, measurable symptom caused by a harmful organism (Zadoks, 1985). 
The resulting yield, obtained in a crop that has been injured by one or several pests, is defined as the actual yield, $\boldsymbol{Y}$ (Rabbinge, 1993): the actual yield, therefore, is the crop yield actually harvested in a farmer's field.

Yield loss (YL), or damage (Zadoks, 1985), represents the difference between the attainable and the actual yield; that is, the yield lost from pests' injuries. Yield loss can be associated to individual pests as well as to multiple pests. The functional relationships between production situation, attainable and actual yields, yield losses and injuries are summarized in Fig. 8.2.

Yield loss is frequently expressed as the fraction (percentage) of the attainable yield lost to pest injuries. It is then called relative yield loss $(\boldsymbol{R Y L})$, and is computed as: $R Y L=100 \times[(Y a-Y) / Y a]$.

The relationship between injury levels and the yield loss they cause is one important quantitative component analyzed when addressing yield losses. This relationship is called a damage function.

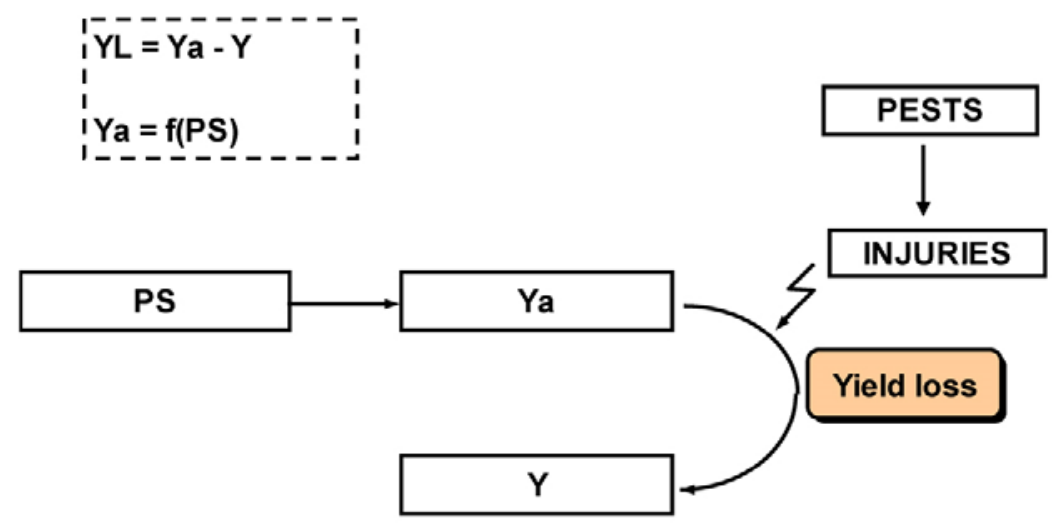

Figure 8.2. Relationships between production situation (PS), attainable yield (Ya), and actual yield (Y); yield loss (YL) (Willocquet et al., 1998).

Production situations may correspond to varying levels of attainable and actual yields, as illustrated in Fig. 8.3. For example:

- two different production situations (i.e., combinations of yield-defining and yield-limiting factors) may correspond to the same level of $\mathrm{Ya}$, but to different levels of yield losses (i.e., different combinations of pest injuries), and therefore to different actual yields (PS1 and PS2);

- two production situations may correspond to different levels of $Y a$, but to the same level of actual yield (because yield losses are different: PS2 and PS3); 
- two production situations may correspond to different levels of $Y a$ and actual yield (PS3 and PS4), the ranking of yield levels between the two production situations being the same $\left(\mathrm{Ya}_{\mathrm{PS} 3}>\right.$ $\mathrm{Ya}_{\mathrm{PS} 4}$ and $\left.\mathrm{Y}_{\mathrm{PS} 3}>\mathrm{Y}_{\mathrm{PS} 4}\right)$;

- two production situations may correspond to different levels of $Y a$ and actual yield (PS4 and PS5), the ranking of yield levels between the two production situations being opposite ( $\mathrm{Ya}_{\mathrm{PS} 4}>$ $\mathrm{Ya}_{\mathrm{PS} 5}$ and $\mathrm{Y}_{\mathrm{PS} 4}<\mathrm{Y}_{\mathrm{PS} 5}$ ).

This diversity of possibilities implies that the quantification of the relative role of the different factors determining the actual yield is a first step when aiming at improving agrosystems' performance.

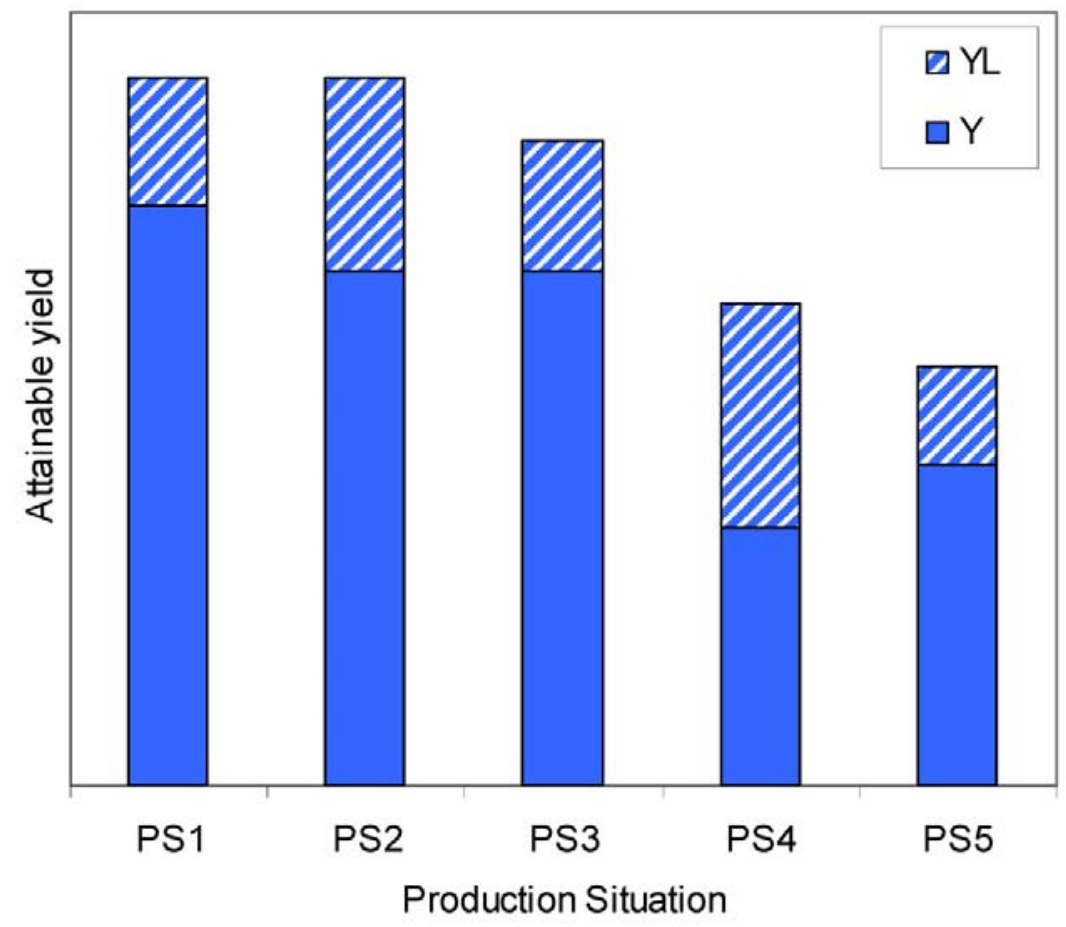

Figure 8.3. An illustration of yield levels in a range of production situations. The concept of damage mechanisms

\section{The concept of damage mechanisms}

Damage functions, which quantify the relationships between injuries and yield losses (Zadoks, 1985), can be determined experimentally. They can also be determined from crop loss simulation models, because, as processes, the damage functions represent processes that are underpinned by subprocesses: damage mechanisms (DM). In these models, the processes involved in plant growth are represented, as well as DMs. Damage mechanisms refer to the processes involved in crop growth that 
are affected by a harmful agent. Different mechanisms can be described (Rabbinge and Rijsdijk, 1981; Boote et al., 1983). The main categories of DMs are listed in Table 8.1.

Table 8.1. Damage mechanisms of crop pest injuries ${ }^{\mathrm{a}}$

\begin{tabular}{|c|c|c|c|}
\hline $\begin{array}{l}\text { Damage } \\
\text { mechanism }\end{array}$ & Physiological effect & $\begin{array}{l}\text { Effect in a crop } \\
\text { growth model }\end{array}$ & Examples of pests \\
\hline Light stealer & $\begin{array}{l}\text { Reduces the } \\
\text { intercepted radiation }\end{array}$ & Reduces the green LAI & $\begin{array}{l}\text { Pathogens producing } \\
\text { lesions on leaves }\end{array}$ \\
\hline $\begin{array}{l}\text { Leaf senescence } \\
\text { accelerator }\end{array}$ & $\begin{array}{l}\text { Increases leaf } \\
\text { senescence, causes } \\
\text { defoliation }\end{array}$ & $\begin{array}{l}\text { Reduces the biomass of } \\
\text { leaves by increasing the } \\
\text { rate of leaf senescence }\end{array}$ & $\begin{array}{l}\text { Foliar pathogens such as } \\
\text { leaf-spotting pathogens, } \\
\text { downy mildews }\end{array}$ \\
\hline Tissue consumer & $\begin{array}{l}\text { Reduces the tissue } \\
\text { biomass }\end{array}$ & $\begin{array}{l}\text { Outflows from } \\
\text { biomasses of the } \\
\text { injured organs }\end{array}$ & Defoliating insects \\
\hline Stand reducer & $\begin{array}{l}\text { Reduces the number } \\
\text { and biomass of plants }\end{array}$ & $\begin{array}{l}\text { Reduces biomass of all } \\
\text { organs }\end{array}$ & Damping-off fungi \\
\hline $\begin{array}{l}\text { Photosynthetic rate } \\
\text { reducer }\end{array}$ & $\begin{array}{l}\text { Reduces the rate of } \\
\text { carbon uptake }\end{array}$ & Reduces the RUE & $\begin{array}{l}\text { Viruses, root-infecting } \\
\text { pests, stem-infecting pests, } \\
\text { some foliar pathogens }\end{array}$ \\
\hline Turgor reducer & $\begin{array}{l}\text { Disrupts xylem and } \\
\text { phloem transport }\end{array}$ & $\begin{array}{l}\text { Reduces the RUE, } \\
\text { accelerates leaf } \\
\text { senescence }\end{array}$ & Vascular, wilt pathogens \\
\hline Assimilate sapper & $\begin{array}{l}\text { Removes soluble } \\
\text { assimilates from host }\end{array}$ & $\begin{array}{l}\text { Outflows assimilates } \\
\text { from the pool of } \\
\text { assimilates }\end{array}$ & $\begin{array}{l}\text { Sucking insects, e.g. } \\
\text { aphids, some planthoppers, } \\
\text { biotrophic fungi exporting } \\
\text { assimilates from host cells }\end{array}$ \\
\hline
\end{tabular}

Damage mechanisms have been experimentally measured for many pests, for example on groundnut rust (Savary et al., 1990), rice leaf blast (Bastiaans, 1991), bean diseases (Bassanezi et al., 2001; Lopes et al., 2001), and wheat Septoria tritici blotch (Robert et al., 2006). Such quantification allows a better understanding of the underlying mechanisms of the effects of pests on crop growth. 
The use of DM parameters can serve at least three purposes:

- DMs can be incorporated into models simulating components of crop growth, e.g., canopy photosynthesis (Bastiaans and Kropff, 1993), and assimilate partitioning (Bancal et al., 2012).

- DMs can be incorporated in crop growth simulation models in order to simulate their effect of crop growth and yield. How to implement this will be described in section 8.4, and examples from the literature are given in the introduction of this chapter.

- parameters for DMs can also be used to compare host plant resistance levels amongst genotypes of a given crop (e.g., Bastiaans and Roumen, 1993).

The use of damage mechanism parameters illustrates again one important characteristic of mechanistic simulation modeling, that is, the mobilization of parameters that have been acquired experimentally. Therefore, there is no disconnection, but, to the contrary, a complete loop from experimental data to model (parameters) and from model to experiments (experimentally measured system's response).

The effects of pests on crop growth using the RI-RUE framework

The damage mechanisms described above can be linked to the RI-RUE concepts described in the previous chapter. Johnson (1987) grouped damage mechanisms in two broad categories, according to their major effect on RI (the first four damage mechanisms: light stealers, leaf senescence accelerators, tissue consumers, and stand reducers) and RUE (the last three damage mechanisms: photosynthetic rate reducer, turgor reducers, and assimilate sappers).

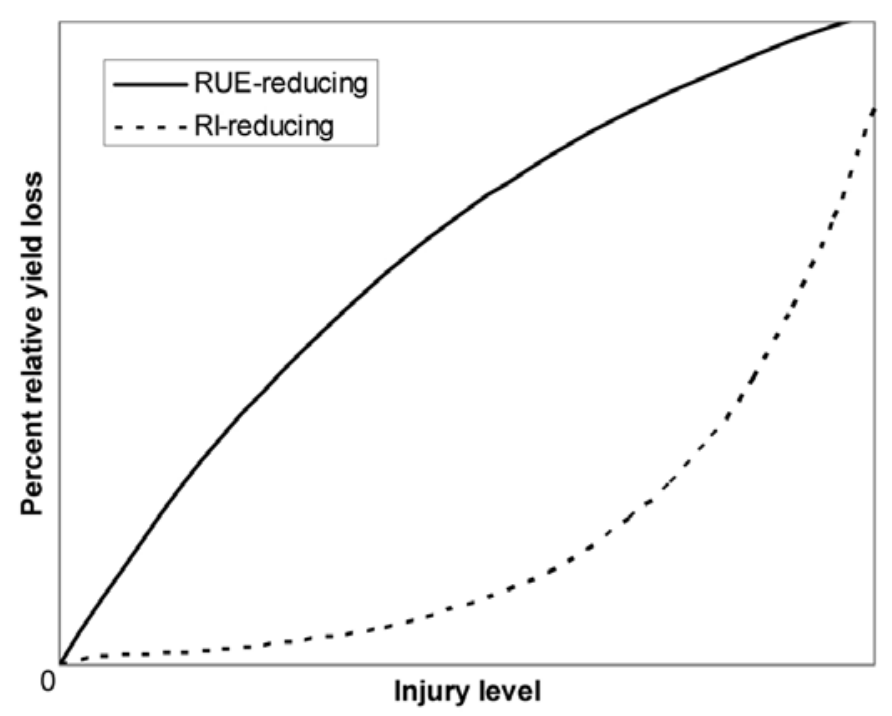

Figure 8.4. Types of damage functions corresponding to RI-reducing and RUE-reducing pests (derived from Johnson, 1987). 
Using a potato crop growth simulation model including damage mechanisms for several pests, Johnson (1987) exemplified the effects of injuries on yield losses according to their yield-reducing effects (through a reduction of RI or of RUE; Fig. 8.4).

Because of Beer's law relationship between LAI and RI, a pest reducing the LAI will have a small reducing effect on yield at low pest intensity. On the other hand, RUE-reducing pests will have a large effect even at low pest intensity, and this effect will decrease (relatively) as pest intensity (and injury) increases.

Grouping pests according to their effects on RI and RUE may be useful for crop loss assessment and disease (pest) management. Analyzing these relationships (damage function, damage mechanism, RI-RUE-reducing effect) allows one to:

(1) address this type of research question in a synthetic way, while

(2) still accounting for the underlying biological mechanisms.

These underlying mechanisms involve questions pertaining to (1) the impact of pests on yield losses, (2) the injury thresholds for pest management, and (3) multiple-pest systems (Johnson, 1987). This approach has been used to analyze many, diverse, pathosystems. It remains very appealing when analyzing interactions between pests, yield, and production situations (Savary et al., 2006). The simplicity of the framework may provide an appealing way for analyses incorporating other factors, e.g., decision making or incorporating other species such as antagonists.

\section{A simple crop growth simulation model for actual growth and yield, and yield losses - GENEPEST}

\section{Stages to simulate yield losses, and possible outcomes}

Simulation of crop growth and yield affected by pest injuries can be made by incorporating into a crop growth model (such as GENECROP) the damage mechanisms corresponding to the injuries addressed. We shall call this new model GENEPEST. A complete listing of the program can be found in Appendix 8.1.

A three-stage approach then allows the simulation of yield losses:

1. Simulation of non-injured growth, enabling one to model the attainable growth and attainable yield $(\mathrm{Ya})$ of a crop under a given production situation. By definition, all injury levels are then set to zero.

2. Simulation of growth under specified levels of injuries in order to model the actual growth and actual yield $(Y)$.

3. Computation of yield losses, that is, the difference between simulated attainable and actual yields. 
Note 1. Simulating growth and yield with levels of injuries corresponding to improved pest management $(\mathrm{Ym})$ allows estimating yield that would be gained on the actual yield $(Y)$ from this improvement in pest management $(Y m-Y)$, thus providing a basis to guide strategic decisions such as research priorities in pest management.

Note 2. Yield losses can be simulated for a range of production situations, by setting the crop drivers (i.e., parameters and interpolation functions for crop growth) to values corresponding to each production situation, and proceeding to the three stages described above.

Note 3. Yield losses can be simulated for injuries considered individually and for combinations of injuries (i.e., grouped as pre-defined injury profiles), thus allowing ranking injuries according to their importance in terms of the yield losses they cause. Such results can help in ranking crop health problems and, again, help for guiding research priorities in pest management.

\section{Incorporating damage mechanisms into a crop growth model}

The damage mechanisms given in Table 8.1 can be incorporated in the crop growth model, GENECROP described in the previous chapter, leading to the GENEPEST model (Fig. 8.5).

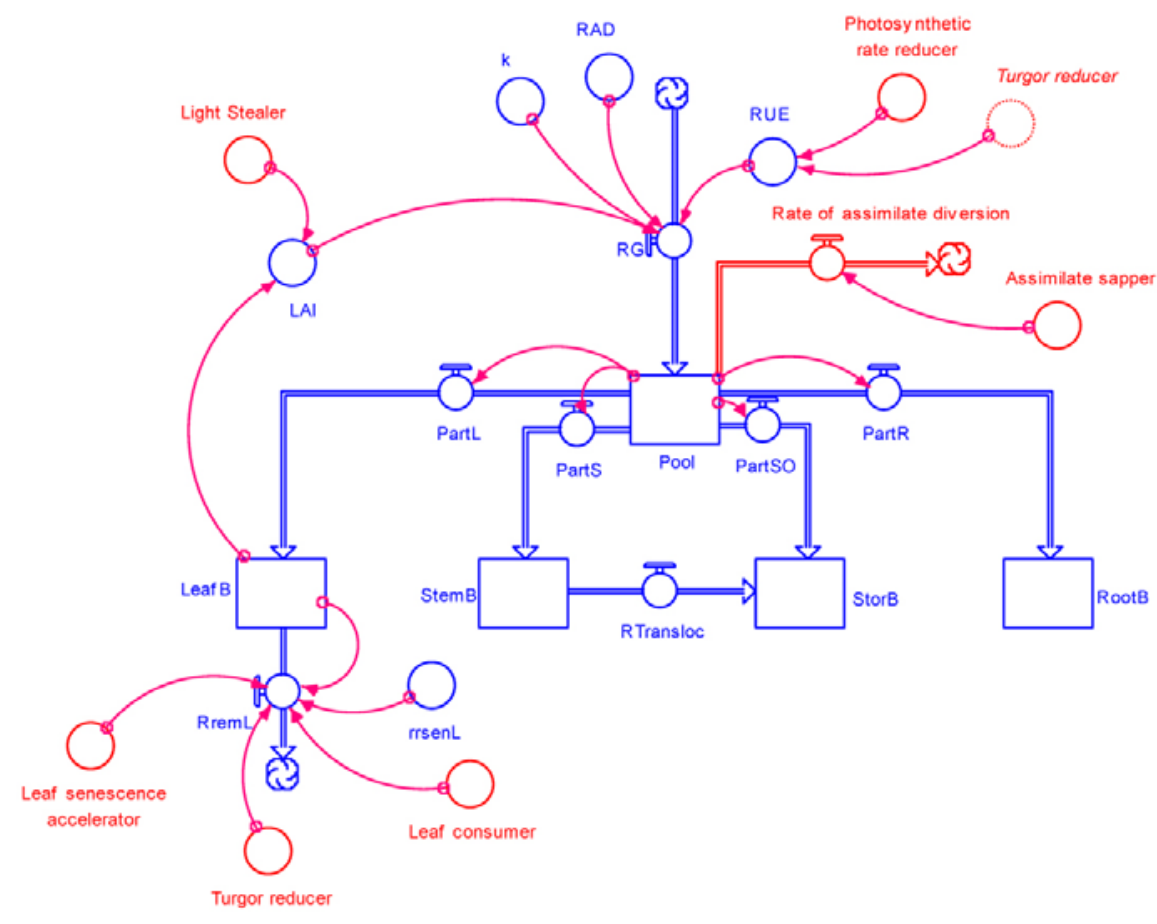

Figure 8.5. GENEPEST: general structure of a crop growth model incorporating damage mechanisms from pest injuries. 
Stand reducers are not included in Fig 8.5 in order to avoid crowding the diagram. Stand reducers would affect the biomass of all organs, and would be reflected by rates of reduction of biomass for all for organs.

Fig. 8.5 indicates that:

(1) all damage mechanisms can be accounted for in GENEPEST,

(2) the different damage mechanisms correspond in general to effects on different processes (rates) or on different variables,

(3) different damage mechanisms however can affect the same process (i.e., leaf consumers and leaf senescence accelerators cause a reduction in [green] leaf biomass, and

(4) a damage mechanism can affect more than one process or variable, as in the case of turgor reducers.

Damage mechanisms are now considered with examples from varying pests in order to illustrate how damage mechanisms can be coupled to a crop growth model.

\section{$\underline{\text { Light stealers }}$}

Light stealers decrease the area of green LAI. This typically corresponds to leaf diseases. Thus, equation (7.6) in Chapter 7:

$$
L A I_{t}=S L A_{t} \times L E A F B_{t}
$$

becomes, for one leaf disease, LD1:

$$
L A I_{t}=S L A_{t} \times L E A F B_{t} \times R F_{L D 1 t}
$$

where $R F$ stands for the 'Reduction Factor' associated to the injury caused by leaf disease 1 . Note that this reduction factor is dynamic, as indicated by the $t$ index. In the case of a foliar disease, which produces lesions that decrease the green LAI, equation (8.1) can be simply written as:

$$
L A I_{t}=S L A_{t} \times L E A F B_{t} \times\left(1-x_{L D 1 t}\right)
$$


where $x_{L D I t}$ is the disease severity of LD1 (i.e., the fraction of leaves covered by lesions varying between 0 and 1) at time $t$. Equation (8.1) reflects the decrease in (green) LAI caused by disease, which corresponds to the leaf area covered by lesions and not photosynthesizing any more. Again, the reduction in LAI is dynamic, as disease severity can be made to vary over the course of an epidemic.

If we consider three leaf diseases LD1, LD2, LD3, equation (8.2) becomes:

$$
L A I_{t}=S L A_{t} \times L E A F B_{t} \times\left(1-x_{L D 1 t}\right) \times\left(1-x_{L D 2 t}\right) \times\left(1-x_{L D 3 t}\right) .
$$

The underlying hypotheses of this equation are that (1) decreases in LAI can be due to one disease only (overlapping of lesions from two different diseases will reduce the LAI only once), and (2) the three diseases are randomly distributed in the crop canopy.

\section{$\underline{\text { Leaf senescence accelerators and tissue (leaf) consumers }}$}

Leaf senescence accelerators and leaf consumers generally refer to different pests, the former typically corresponding to pathogens, and the second to insect defoliators. From a modeling point of view, they are however handled together and in the same manner here, because they correspond to the same effect on crop growth, i.e., a reduction in leaf dry biomass. The incorporation of these effects into the model is first described in the case of leaf senescence accelerators and then in the case of leaf consumers.

Leaf senescence accelerators have the same physiological effect as physiological senescence, and are therefore accounted for in the crop growth model in the same way as physiological senescence. So, equation 7.18 in Chapter 7:

$$
L E A F B_{t+\Delta t}=L E A F B_{t}+\left(\left(P A R T L_{t}-R S E N L_{t}\right) \times \Delta t\right)
$$

becomes:

$$
\operatorname{LEAFB}_{t+\Delta t}=\operatorname{LEAFB}_{t}+\left(\left(\mathrm{PARTL}_{t}-\mathrm{RSENL}_{t}-\mathrm{RSENIN1}_{t}\right) \times \Delta t\right)
$$

where RSENIN1 is the rate of leaf senescence caused by injury. It is convenient to establish a relationship between RSENIN1 and injury level by expressing this rate of senescence as the product of a relative rate of senescence by the leaf dry biomass: 


$$
\operatorname{RSENIN1}_{t}=\operatorname{RRSENIN1}_{t} \times \operatorname{LEAFB}_{t}
$$

with:

$$
\text { RRSENIN } 1_{t}=\text { alpha } \times I N 1_{t}
$$

Equations (8.5) and (8.6) simply mean that in the case of leaf senescence caused by an injury, the fraction of leaf senesced is proportional to the intensity of the injury. Injury can be expressed as disease severity (i.e., a fraction between 0 and 1 ). The magnitude of the effect of injury on senescence corresponds to the parameter alpha, which needs to be measured experimentally.

An important example of tissue consumers is the case of defoliating insects, which decrease the leaf biomass by eating leaves or fractions of leaves. This type of damage mechanism can be reflected in equation (7.18) by reducing the leaf biomass as a result of consumption by defoliating insects:

$$
L E A F B_{t+\Delta t}=L E A F B_{t}+\left(\left(P A R T L_{t}-R S E N L_{t}-R D E F_{t}\right) \times \Delta t\right)
$$

where $R D E F_{t}$ is the rate of defoliation. In the same way as for senescence accelerators, a relationship can be established between the rate of defoliation and the injury:

$$
R D E F_{t}=R F D E F_{t} \times L E A F B_{t}
$$

with $R F D E F_{t}$ is the rate of increase in fraction of leaf area damaged by defoliation. This rate can be derived from successive assessments of the fraction of leaf area defoliated.

When combining effects of senescence accelerators and leaf consumers, the following hypothesis is made: leaf consumers do not damage leaf tissues that are senesced, and leaf senescence cannot occur on defoliated parts of leaves. The combined effects of these two damages are therefore additive and can be written as:

$$
L E A F B_{t+\Delta t}=L E A F B_{t}+\left(\left(P A R T L_{t}-R S E N L_{t}-R_{S E N I N 1_{t}}-R D E F_{t}\right) \times \Delta t\right)
$$




\section{Photosynthetic rate reducers}

Photosynthetic rate reducers can be incorporated in a crop growth model such as GENECROP (chapter 7) by decreasing the RUE. In crop growth models describing in more detail the photosynthesis processes, the effect of photosynthetic rate reducers would be reflected by a reduction in, for example, the initial light use efficiency of single leaves, and/or a reduction in the maximum rate of photosynthesis, and/or an increase in dark respiration (e.g., Rossing et al., 1992).

In GENECROP, equation 7.5 in Chapter 7:

$$
R G_{t}=R U E_{t} \times R A D_{t} \times\left(1-e^{-k \times L A I_{t}}\right)
$$

becomes:

$$
R G_{t}=R U E_{t} \times R A D_{t} \times\left(1-e^{-k \times L A I_{t}}\right) \times R F_{P R 1 t}
$$

In the case of light stealers such as (leaf-spotting) foliar diseases, the relationship between the reduction factor and the level of injury is straightforward: the reduction in green LAI corresponds to disease severity and $R F=1-x=1$ - severity.

When addressing photosynthetic rate reducers, the relationship between the reduction factor and the level of injury is less straightforward, and often needs to be established experimentally. Two examples corresponding to pests which widely differ biologically (a viral disease and a root-infecting disease), but nevertheless cause similar damage mechanisms by reducing the $R U E$, are given below to illustrate how $R F$ can be expressed.

Viral diseases are in general systemic and the virus particles are transported within the plant via its vascular system. Virus infection can reduce the rate of photosynthesis and this can be simply represented by the relationship between the proportion of disease plants and the reduction factor:

$$
R F_{P R 1 t}=1-\left(\text { delta } \times V I R_{t}\right)
$$

where delta is a parameter ranging between 0 and 1, which represents the magnitude in the effect of viral infection to reduce the $R U E$, and $V I R_{t}$ is the proportion of diseased plants. The parameter delta needs to be measured with specific experiments. 
Root-infecting diseases cause injuries, which directly affect the functioning of infected roots, and therefore the amount of water and nutrients absorbed by the roots. This in turn causes a reduction in $R U E$. A relationship between the disease level and $R F$ can be written as:

$$
R F_{P R 2 t}=1-\left(\text { gamma } \times R D I S_{t}\right)
$$

where, similarly to equation (8.11), gamma is a parameter ranging between zero and 1, which represents the magnitude in the effect of root infection to reduce the $R U E$, and $R D I S_{t}$ is the proportion of roots infected by the pathogen. The parameter gamma needs to be measured with specific experiments.

Accounting for the combined effects of the two above pests can be done by multiplying the reduction factors, which reflects (1) the interactions between both pests in their effect on RUE and (2) the hypothesis of random distribution of both pests. Equation (8.10) becomes:

$$
R G_{t}=R U E_{t} \times R A D_{t} \times\left(1-e^{-k \times L A I_{t}}\right) \times R F_{P R 1 t} \times R F_{P R 2 t}
$$

\section{$\underline{\text { Assimilate sappers }}$}

Assimilate sappers uptake assimilates produced from photosynthesis. Two important pest groups cause this type of damage mechanism: insects such as aphids or plant hoppers which are feeding from the phloem sap, and biotrophic fungi such as rusts which are diverting the assimilates to produce fungal organs, especially spores. One could also add a number of plant nematodes, at least those which do not cause tissue necrosis.

The diversion of assimilates is accounted for in the simulation of the dynamics of the pool of assimilates. Equation (7.16) from Chapter 7:

$$
\mathrm{POOL}_{t+\Delta t}=\mathrm{POOL}_{t}+\left[\left(R_{t}-\mathrm{PARTL}_{t}-\mathrm{PARTS}_{t}-\mathrm{PARTR}_{t}-\mathrm{PARTSO}_{t}\right) \times \Delta t\right]
$$

becomes:

$$
\mathrm{POOL}_{t+\Delta t}=\mathrm{POOL}_{t}+\left[\left(R_{t}-\mathrm{PARTL}_{t}-\mathrm{PARTS}_{t}-\mathrm{PARTR}_{t}-\mathrm{PARTSO}_{t}-\mathrm{DIV} \mathrm{V}_{t}\right) \times \Delta t\right]
$$


The amount of assimilates diverted by pests is retrieved from the amount of assimilates partitioned towards organs, and equations (7.12) to (7.15) in Chapter 7 become:

$$
\begin{gathered}
\text { PARTL }_{t}=\left(\text { POOL }_{t}-D I V_{t}\right) \times C P L_{t} \times\left(1-C P R_{t}\right) \\
\text { PARTS }_{t}=\left(\text { POOL }_{t}-D I V_{t}\right) \times C P S_{t} \times\left(1-C P R_{t}\right) \\
\text { PARTR }_{t}=\left(P O O L_{t}-D I V_{t}\right) \times C P R_{t} \\
\text { PARTSO }_{t}=\left(\text { POOL }_{t}-D I V_{t}\right) \times C P S O_{t} \times\left(1-C P R_{t}\right)
\end{gathered}
$$

Again, a relationship needs to be established experimentally between the amount of assimilates diverted by the pest and the level of injury. In the case of insects, this corresponds to the sapping (or sucking) rate, and can depend on the crop development stage and the insect development stage (or weight). The diversion rate can be written as:

$$
\operatorname{DIVINS}_{t}=\operatorname{rrsap}_{t} \times \text { bmperins }_{t} \times \text { NBINS }_{t}
$$

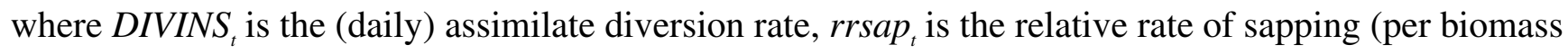
of insect and per day), bmperins $_{t}$ is the biomass of an individual insect, and NBINS is the number of

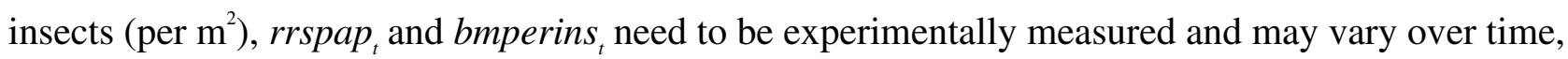
and $N B I N S_{t}$ is the insect pest driving function, which may vary over time, and represents the dynamics of insect density.

In the case of biotrophic fungi, the relationships between disease intensity and the diversion of assimilates can be done according to the carbohydrate uptake for spore production, the number of spores produced per lesion per day, and the lesion size:

$$
\operatorname{DIVBIOT}_{t}=\text { rruptake } \times N B L E S_{t}
$$

with:

$$
N B L E S_{t}=\frac{L A I_{t} \times S E V B I O T_{t}}{\text { lesize }}
$$


where rruptake is the rate of carbohydrate uptake per lesion per day; and the number of lesions is derived from disease severity, SEVBIOT (pest driver), lesion area (lesize), and LAI.

When combining the two above pests, a simple hypothesis corresponds to the independence between both pests and their injuries, leading equation (8.14) to become:

$\mathrm{POOL}_{t+\Delta t}=\mathrm{POOL}_{t}+\left[\left(R_{t}-\mathrm{PARTL}_{t}-\mathrm{PARTS}_{t}-\mathrm{PARTR}_{t}-\mathrm{PARTSO}_{t}-\mathrm{DIVINS}_{t}-\mathrm{DIVBIOT}_{t}\right) \times \Delta t\right]$ $(8.22)$

and replacing $P O O L_{t}-D I V_{t}$ by $P O O L_{t}-D_{I V I N S}-D_{t} I V I O T_{t}$ in equations (8.15) to (8.18).

Turgor reducer

Damage mechanisms associated with turgor reducers have been addressed when considering RUE reducers and leaf senescence accelerators. They will therefore not be illustrated specifically in this chapter. Accounting for turgor reducers will however be illustrated in the next chapter.

Important note: for the sake of simplicity, the incorporation of damage mechanisms into a crop growth simulation model has been described above for each damage mechanism, one at a time.

Individual crop pests can, however, cause more than one type of damage. This will be illustrated in the GENEPEST model, and in the description of RICEPEST and WHEATPEST models in the next chapter.

\section{Model parameters for damage mechanisms}

The parameters needed to simulate damage mechanisms are derived from experiments. Two main types of parameters can be considered:

(1) parameters which represent the magnitude of the impact of pest injuries on the crop physiological processes:

alpha (leaf senescence accelerator);

delta (virus disease - effect on RUE);

gamma (root-infecting disease - effect on RUE);

rrsap (insect sapper - rate of sapping); and

rruptake (biotrophic pathogen - rate of assimilate uptake).

(2) parameters corresponding to ecological characteristics of the pests, which are needed to determine a relationship between the damage mechanism and the level of injury: 
bmperins (biomass of an individual insect); and

lesize (area of a lesion).

The following values are set in GENEPEST (again, these have to be experimentally measured for a given pest).

alpha $=0.076$ day $^{-1}$ (case of rice sheath blight; Willocquet et al., 2000)

delta $=0.35$ (case of wheat BYDV; Willocquet et al., 2008)

gamma $=1$ (case of wheat take-all; Willocquet et al., 2008)

rrsap $=1 \mathrm{mg} \cdot \mathrm{mg}^{-1} \cdot \operatorname{day}^{-1}$ (arbitrarily chosen value)

rruptake $=4.62 \times 10^{-6}$ day $^{-1}$ (case of wheat brown or leaf rust; Willocquet et al., 2008)

bmperins $=0.5 \mathrm{mg}$ (arbitrarily chosen value)

lesize $=10^{-6} \mathrm{~m}^{2}$ (case of wheat brown or leaf rust; Willocquet et al., 2008)

\section{Model drivers for pests injuries}

The damage mechanisms described above have been implemented into GENEPEST by considering several pests, which provide a combination of the damage mechanisms described previously. The pests considered are described in Table 8.2.

Table 8.2. Examples of pests accounted for in GENEPEST ${ }^{\mathrm{a}}$

\begin{tabular}{|c|c|c|c|c|}
\hline Pest name & Pest type & Driving function & Damage mechanism 1 & Damage mechanism 2 \\
\hline LD1 & Foliar pathogen & \multirow{3}{*}{$\begin{array}{l}\text { Disease severity } \\
\text { (fraction leaf area } \\
\text { infected) }\end{array}$} & light stealer & \\
\hline LD2 & Foliar pathogen & & light stealer & $\begin{array}{l}\text { leaf senescence } \\
\text { accelerator }\end{array}$ \\
\hline LD3 & Foliar pathogen & & light stealer & assimilate sapper \\
\hline VIR & Virus & $\begin{array}{l}\text { Disease incidence } \\
\text { (fraction plants } \\
\text { infected) }\end{array}$ & $\begin{array}{l}\text { photosynthetic rate } \\
\text { reducer }\end{array}$ & \\
\hline $\mathrm{DEF}$ & $\begin{array}{l}\text { Defoliating } \\
\text { insect }\end{array}$ & $\begin{array}{l}\text { Daily fraction of leaf } \\
\text { area defoliated }\end{array}$ & tissue (leaf) consumer & \\
\hline RDIS & $\begin{array}{l}\text { Root-infecting } \\
\text { pathogen }\end{array}$ & $\begin{array}{l}\text { Disease severity } \\
\text { (fraction root } \\
\text { infected) }\end{array}$ & $\begin{array}{l}\text { photosynthetic rate } \\
\text { reducer }\end{array}$ & \\
\hline NBINS & Sucking insect & $\mathrm{Nb}$ of insects $\left(\right.$ per $\left.\mathrm{m}^{2}\right)$ & assimilate sapper & \\
\hline
\end{tabular}




\section{Simulations}

The STELLA model GENEPEST.STMX will allow you to:

- explore the model structure and equations,

- explore the model inputs, especially the driving functions of the different pests included

- explore the model outputs, and

- run the model with varying levels of injury, which will allow you to explore:

- the effects of individual injuries on crop growth and yield

- the effects of combined injuries on crop growth and yield

\section{Summary}

This chapter describes:

- Concepts and definitions related to yield levels, production situations and injuries

- The concept of damage mechanism

- The effects of pests on crop growth within the RI-RUE framework

- How damage mechanisms are captured in a quantitative and dynamic way into a generic simulation model, GENEPEST.

- Provides the equations, parameters, and flowchart of GENEPEST.

- Includes the STELLA file, which can be used to explore the model structure and the effect of injuries, individually or in combination, on the simulated dynamics of crop growth.

\section{References}

Bancal, M. O., Hansart, A., Sache, I., and Bancal, P. 2012. Modelling fungal sink competitiveness with grains for assimilates in wheat infected by a biotrophic pathogen. Ann. Bot. 110:113-123.

Bassanezi, R. B., Amorim, L., Bergamin Filho, A., Hau, B., and Berger, R. D. 2001. Accounting for photosynthetic efficiency of bean leaves with rust, angular leaf spot and anthracnose to assess crop damage. Plant Pathol. 50:443-452.

Bastiaans, L. 1991. Ratio between virtual and visual lesion size as a measure to describe reduction in leaf photosynthesis of rice due to blast. Phytopathology 81:611-615. 
Bastiaans, L. 1993. Effects of leaf blast on growth and production of a rice crop. 2. Analysis of the reduction in dry matter production, using two models with different complexity. Neth. J. Plant Pathol. 99 (suppl3):19-28.

Bastiaans, L., and Kropff, M. J. 1993. Effects of leaf blast on photosynthesis of rice. 2. Canopy photosynthesis. Neth. J. Plant Pathol. 99:205-217.

Bastiaans, L., and Roumen, E. C. 1993. Effect on leaf photosynthetic rate by leaf blast for rice cultivars with different types and levels of resistance. Euphytica 66:81-87.

Boote, K. J., Jones, J. W., Mishoe, J. W., and Berger, R. D. 1983. Coupling pests to crop growth simulators to predict yield reductions. Phytopathology 73:1581-1587.

Chiarappa, L. 1971. Crop Loss Assessment Methods: FAO Manual on the Evaluation and Prevention of Losses by Pests, Diseases and Weeds. FAO - Commonwealth Agricultural Bureau, Farnham Royal, UK.

Johnson, K. B. 1987. Defoliation, disease, and growth: a reply. Phytopathology 77:1495-1497.

Johnson, K. B. 1992. Evaluation of a mechanistic model that describes potato crop losses caused by multiple pests. Phytopathology 82:363-369.

Loomis, R. S., and Adams, S. S. 1983. Integrative analyses of host-pathogen relations. Annu. Rev. Phytopathol. 21:341-362.

Lopes, D. B., and Berger, R. D. 2001. The effects of rust and anthracnose on the photosynthesis competence of diseased bean leaves. Phytopathology 91:212-220.

Madden, L. V., Hughes, G., and Irwin, M. E. 2000. Coupling disease-progress-curve and time-ofinfection functions for predicting yield loss of crops. Phytopathology 90:788-800.

Rabbinge, R. 1993. The ecological background of food production. Pages 2-29 in: Crop Protection and Sustainable Agriculture. Ciba Foundation 77. D. J. Chadwick and J. Marsh, eds. John Wiley \& Sons, Chichester, UK.

Rabbinge, R., Ward, S. A., and Van Laar, H. H., eds. 1989. Simulation and Systems Management in Crop Protection. Pudoc, Wageningen, The Netherlands.

Rabbinge, R., and Rijsdijk, P. H. 1981. Disease and crop physiology: a modeler's point of view. Pages 201-220 in: Effects of Disease on the Physiology of the Growing Plants. P. G. Ayres, ed. Cambridge Univ. Press, Cambridge, UK.

Rabbinge, R., and Vereyken, P. H. 1980. The effects of diseases or pests upon host. Z. Pflanzenk. Pflanzensch. 87:409-422. 
Robert C., Bancal, M. O., Lannou, C., and Ney, B. 2006. Quantification of the effects of Septoria tritici blotch on wheat leaf gas exchange with respect to lesion age, leaf number, and leaf nitrogen status. J. Exp. Bot. 57:225-234.

Rossing, W. A. H., van Oijen, M., van der Werf, W., Bastiaans, L., and Rabbinge, R., 1992. Modelling the effects of foliar pests and pathogens on light interception, photosynthesis, growth rate and yield of field crops. Pages 161-180 in: Pests and Pathogens. Plant Responses to Foliar Attack. P. G. Ayres, ed. Bios Scientific, Oxford, UK.

Savary, S., De Jong, P. D., Rabbinge, R., and Zadoks, J. C. 1990. Dynamic simulation of groundnut rust: a preliminary model. Agric. Syst. 32:113-141.

Savary, S., and Zadoks, J. C. 1992. An analysis of crop loss in the multiple pathosystem groundnut rust - late leaf spot. II . A study of the interactions between diseases and crop intensification in factorial experiments. Crop Prot. 11:110-120.

Savary, S., Teng, P. S., Willocquet, L., and Nutter, F. W., Jr. 2006. Quantification and modeling of crop losses: a review of purposes. Annu. Rev. Phytopathol. 44:89-112.

Teng, P. S., ed. 1987. Crop Loss Assessment and Pest Management. APS Press, St Paul MN.

Van Ittersum, M. K., and Rabbinge, R. 1997. Ecology for analysis and quantification of agricultural input-output combinations. Field Crops Res. 52:197-208.

Willocquet, L., Savary, S., Fernandez, L., Elazegui, F. A., and Teng, P. S. 1998. Simulation of yield losses caused by rice diseases, insects, and weeds in tropical Asia. IRRI Discussion Paper Series no 34. IRRI, Los Baños, Philippines.

Willocquet, L., Savary, S., Fernandez, L., Elazegui, F. A., and Teng, P. S. 2000. Development and evaluation of a multiple-pest, production situation specific, simulation model of rice yield losses in tropical Asia. Ecol. Model. 131:133-159.

Willocquet, L., Aubertot, J. N., Lebard, S., Robert, C., Lannou, C., and Savary, S. 2008. Simulating multiple pest damage in varying winter wheat production situations. Field Crops Res. 107:12-28.

Wit, C. T. de, and Penning de Vries, F. W. T. 1982. L'analyse des systèmes de production primaire. Pages 20-27 in: La Prodctivité de Pâturages Sahéliens. F. W. T. Penning de Vries and M. A. Djiteye, eds. Agricultural Research Reports 918. Pudoc, Wageningen.

Zadoks, J. C. 1985. On the conceptual basis of crop loss assessment: the threshold theory. Annu. Rev. Phytopathol. 23:455-473.

Zadoks, J. C., and Schein, R. D. 1979. Epidemiology and Plant Disease Management. Oxford University Press, New York, USA. 


\section{Exercises and questions}

1. Give examples of pests in wheat categorized by damage mechanism, following Table 8.1.

2. Indicate which of the following statement is (are) correct
a. yield loss is the difference between attainable and actual yield
b. yield loss is the difference between potential and attainable yield
c. a yield reducing factor may be associated to different injury mechanisms
d. a given damage mechanism can affect different physiological processes

3. A light stealer affects
a. the RUE
b. the partitioning towards organs
c. the leaf biomass
d. the LAI

4. Acceleration of leaf senescence affects
a. the RUE
b. the partitioning towards organs
c. the leaf biomass
d. the LAI

5. A possible unit for the relative rate of leaf senescence is
a. $g \cdot g^{-1} \cdot d a y^{-1}$
b. $\mathrm{g} \cdot \mathrm{day}^{-1}$
c. $\mathrm{g} \cdot \mathrm{g}^{-1}$
d. $\mathrm{g} \cdot \mathrm{m}^{-2} \cdot \mathrm{day}^{-1}$

Answers to exercises and questions

1. pests by damage mechanism in wheat:

b. light stealer: weeds, Septoria blotch;

c. leaf senescence accelerator: Septoria blotch;

d. tissue consumer: many defoliating insects (e.g., Lema spp.); 
e. stand reducer: many soil pathogens: take-all pathogen (e.g., Gaeumannomyces tritici); weeds; barley yellow dwarf virus disease;

f. photosynthetic rate reducer: barley yellow dwarf virus disease; Septoria blotch;

g. turgor reducer: eyespot pathogen (Rhizoctonia spp.);

h. Assimilate sappers: rust pathogens (stripe [yellow], leaf [brown], and stem rust); aphids.

2. a: yield loss is the difference between attainable and actual yield, and c: a yield reducing factor may be associated to different injury mechanisms

3. a: the LAI.

4. c: the leaf area biomass

5. a: $g \cdot g^{-1} \cdot d a y^{-1}$.

Appendix 8.1. Program listing of GENEPEST

LeafB $(\mathrm{t})=\operatorname{LeafB}(\mathrm{t}-\mathrm{dt})+(\operatorname{PartL}-\mathrm{RSenL}) * \mathrm{dt}$

INIT LeafB $=10$

INFLOWS:

PartL $=$ CPL $*$ (Pool-rdiv)

OUTFLOWS:

RSenL $=(($ rrsen $+($ alpha*LD2 $)+$ RFDEF $) *$ LeafB $)$

$\operatorname{MaxStemb}(\mathrm{t})=\operatorname{MaxStemb}(\mathrm{t}-\mathrm{dt})+(\mathrm{rmaxstemb}) * \mathrm{dt}$

INIT MaxStemb $=6$

INFLOWS:

rmaxstemb $=$ PartS

Pool $(\mathrm{t})=$ Pool $(\mathrm{t}-\mathrm{dt})+($ RGrowth - PartS - PartL - PartO - PartR - rdiv $) * d t$

INIT Pool $=0$

INFLOWS:

RGrowth $=$ RAD*RUE*(1-EXP(-k*LAI))*(1-(delta*VIR $) *(1-($ gamma*RDIS $))$

OUTFLOWS:

PartS $=$ CPS $*$ (Pool-rdiv) 
PartL $=$ CPL $*($ Pool-rdiv $)$

$\mathrm{PartO}=\mathrm{CPO} *($ Pool-rdiv $)$

PartR $=\mathrm{CPR} *($ Pool-rdiv $)$

rdiv $=($ rsrsap $*$ bmperins $*$ INS $)+($ rruptake $*$ LAI $*$ LD3/lesize $)$

$\operatorname{REPTIL}(\mathrm{t})=\operatorname{REPTIL}(\mathrm{t}-\mathrm{dt})+(\operatorname{Rmat}-\operatorname{Rmortr}) * d \mathrm{t}$

INIT REPTIL $=0$

INFLOWS:

Rmat $=$ if DVS $<0.8$ or DVS $>1$ then 0 else if VTIL $<$ FST $*$ Totil then 0 else RRMAT*VTIL

OUTFLOWS:

Rmortr $=$ rrmort $*$ REPTIL

$\operatorname{RootB}(\mathrm{t})=\operatorname{RootB}(\mathrm{t}-\mathrm{dt})+(\operatorname{PartR}) * d \mathrm{t}$

INIT RootB $=5$

INFLOWS:

PartR $=$ CPR $*($ Pool-rdiv $)$

$\operatorname{StemB}(\mathrm{t})=\operatorname{StemB}(\mathrm{t}-\mathrm{dt})+($ PartS - RTransloc $) * \mathrm{dt}$

INIT StemB $=6$

INFLOWS:

PartS $=$ CPS $*$ (Pool-rdiv)

OUTFLOWS:

RTransloc $=\operatorname{IF}($ DVS $>1)$ then ddist else 0

$\operatorname{STEMP}(\mathrm{t})=\operatorname{STEMP}(\mathrm{t}-\mathrm{dt})+($ Dtemp $) * d t$

INIT STEMP $=320$

INFLOWS:

Dtemp $=(($ TMAX + TMIN $) / 2)-$ TBASE

$\operatorname{StorB}(\mathrm{t})=\operatorname{StorB}(\mathrm{t}-\mathrm{dt})+($ PartO + RTransloc $) * d t$

INIT StorB $=0$

INFLOWS:

PartO $=$ CPO $*$ (Pool-rdiv $)$

RTransloc $=\operatorname{IF}($ DVS $>1)$ then ddist else 0

$\operatorname{VTIL}(\mathrm{t})=\operatorname{VTIL}(\mathrm{t}-\mathrm{dt})+($ Rtil - Rmat $-\mathrm{Rmrtv}) * \mathrm{dt}$

INIT VTIL $=250$

INFLOWS:

Rtil $=$ PartLS $* S T W *(1-(V T I L / m a x t i l)) * D V E$ 


\section{OUTFLOWS:}

Rmat $=$ if DVS $<0.8$ or DVS $>1$ then 0 else if VTIL $<$ FST $*$ Totil then 0 else RRMAT*VTIL

Rmrtv $=($ rrmort $*$ VTIL)

alpha $=0.076$

bmperins $=0.0005$

$\mathrm{CPL}=\mathrm{CPPL} *(1-\mathrm{CPR})$

$\mathrm{CPO}=\mathrm{CPPO}^{*}(1-\mathrm{CPR})$

$\mathrm{CPS}=(1-\mathrm{CPL}-\mathrm{CPO}) *(1-\mathrm{CPR})$

$\mathrm{DACE}=\mathrm{TIME}+14$

ddist $=0.005 *$ MaxStemb

delta $=0.35$

DVS $=$ if stemp $<$ TFLOW then STEMP/TFLOW ELSE 1+((STEMP-TFLOW)/(TMAT-TFLOW))

$\mathrm{FST}=0.05$

gamma $=1$

grain_yield $=0.85 *$ StorB

INS $=$ pINS $*$ INSn

$\mathrm{k}=0.6$

LAI $=$ LeafB $*$ SLA $*(1-L D 1) *(1-L D 2) *(1-L D 3)$

$\mathrm{LD} 1=\mathrm{pLD} 1 * \mathrm{LD} 1 \mathrm{n}$

$\mathrm{LD} 2=\mathrm{pLD} 2 * \mathrm{LD} 2 \mathrm{n}$

LD3 $=$ pLD3 $*$ LD3n

lesize $=0.000001$

maxtil $=900$

PartLS $=$ PartL+PartS

$\mathrm{pINS}=0$

$\mathrm{pLD} 1=0$

pLD2 $=0$

pLD3 $=0$

$\mathrm{pRDIS}=0$

$\mathrm{pRFDEF}=0$

$\mathrm{pVIR}=0$

$\mathrm{RAD}=17$

RDIS $=$ pRDIS $*$ RDISn 


\section{$\mathrm{RFDEF}=\mathrm{pRFDEF} * \mathrm{RFDEFn}$}

RRMAT $=0.3$

rruptake $=0.00000462$

rsrsap $=1$

$\mathrm{RUE}=1.2$

STW $=20$

TBASE $=8$

TFLOW $=1500$

$\mathrm{TMAT}=2000$

TMAX $=30$

$\mathrm{TMIN}=24$

Totil $=$ VTIL + REPTIL

$\mathrm{VIR}=\mathrm{pVIR} * \mathrm{VIRn}$

$\mathrm{CPPL}=\mathrm{GRAPH}(\mathrm{DVS})$

(0.00, 0.55), (0.1, 0.536), (0.2, 0.521), (0.3, 0.507), (0.4, 0.493), (0.5, 0.479), (0.6, 0.464), (0.7, 0.45), $(0.8,0.3),(0.9,0.15),(1,0.00),(1.10,0.00),(1.20,0.00),(1.30,0.00),(1.40,0.00),(1.50,0.00),(1.60$, 0.00), (1.70, 0.00), (1.80, 0.00), (1.90, 0.00), (2.00, 0.00)

\section{$\mathrm{CPPO}=\mathrm{GRAPH}(\mathrm{DVS})$}

(0.00, 0.00), (0.05, 0.00), (0.1, 0.00), (0.15, 0.00), (0.2, 0.00), (0.25, 0.00), (0.3, 0.00), (0.35, 0.00), $(0.4,0.00),(0.45,0.00),(0.5,0.00),(0.55,0.00),(0.6,0.00),(0.65,0.00),(0.7,0.00),(0.75,0.00),(0.8$, 0.143), (0.85, 0.286), (0.9, 0.429), (0.95, 0.571), (1.00, 0.714), (1.05, 0.857), (1.10, 1.00), (1.15, 1.00), (1.20, 1.00), (1.25, 1.00), (1.30, 1.00), (1.35, 1.00), (1.40, 1.00), (1.45, 1.00), (1.50, 1.00), (1.55, 1.00), $(1.60,1.00),(1.65,1.00),(1.70,1.00),(1.75,1.00),(1.80,1.00),(1.85,1.00),(1.90,1.00),(1.95,1.00)$, $(2.00,1.00)$

\section{$\mathrm{CPR}=\mathrm{GRAPH}(\mathrm{DVS})$}

(0.00, 0.3), (0.1, 0.263), (0.2, 0.225), (0.3, 0.188), (0.4, 0.15), (0.5, 0.112), (0.6, 0.075), (0.7, 0.038), $(0.8,0.00),(0.9,0.00),(1,0.00),(1.10,0.00),(1.20,0.00),(1.30,0.00),(1.40,0.00),(1.50,0.00)$, $(1.60,0.00),(1.70,0.00),(1.80,0.00),(1.90,0.00),(2.00,0.00)$

\section{$\mathrm{DVE}=\mathrm{GRAPH}(\mathrm{DVS})$}

$(0.00,1.00),(0.4,1.00),(0.8,0.00),(1.20,0.00),(1.60,0.00),(2.00,0.00)$

$\mathrm{INSn}=\mathrm{GRAPH}(\mathrm{TIME})$

(0.00, 0.00), (10.0, 0.00), (20.0, 0.00), (30.0, 50.0), (40.0, 100), (50.0, 150), (60.0, 200), (70.0, 150), $(80.0,100),(90.0,50.0),(100,5.00),(110,5.00),(120,5.00)$ 


\section{LD1n = GRAPH(TIME)}

(0.00, 0.00), (10.0, 0.00), (20.0, 0.00), (30.0, 0.004), (40.0, 0.008), (50.0, 0.01), (60.0, 0.007), (70.0, 0.002), (80.0, 0.00), (90.0, 0.00), (100, 0.00), (110, 0.00), (120, 0.00)

\section{$\mathrm{LD} 2 \mathrm{n}=\mathrm{GRAPH}(\mathrm{TIME})$}

(0.00, 0.00), (10.0, 0.00), (20.0, 0.00), (30.0, 0.002), (40.0, 0.005), (50.0, 0.008), (60.0, 0.01), (70.0, 0.008), (80.0, 0.007), (90.0, 0.006), (100, 0.005), (110,0.004), (120, 0.004)

\section{LD3n $=$ GRAPH $($ TIME $)$}

(0.00, 0.00), (10.0, 0.00), (20.0, 0.00), (30.0, 0.003), (40.0, 0.005), (50.0, 0.007), (60.0, 0.009), (70.0, $0.01),(80.0,0.01),(90.0,0.009),(100,0.007),(110,0.005),(120,0.001)$

$\mathrm{RDISn}=\mathrm{GRAPH}(\mathrm{TIME})$

(0.00, 0.00), (10.0, 0.00), (20.0, 0.00), (30.0, 0.001), (40.0, 0.002), (50.0, 0.01), (60.0, 0.01), (70.0, $0.01),(80.0,0.01),(90.0,0.01),(100,0.01),(110,0.01),(120,0.01)$

\section{RFDEFn = GRAPH $($ TIME $)$}

(0.00, 0.00), (10.0, 0.00), (20.0, 0.00), (30.0, 0.00), (40.0, 0.001), (50.0, 0.00), (60.0, 0.00), (70.0, 0.00), (80.0, 0.00), (90.0, 0.00), (100, 0.00), (110, 0.00), (120,0.00)

\section{rrmort $=$ GRAPH $($ DVS $)$}

(0.00, 0.00), (0.1, 0.00), (0.2, 0.00), (0.3, 0.00), (0.4, 0.00), (0.5, 0.02), (0.6, 0.02), (0.7, 0.02), (0.8, 0.02), (0.9, 0.02), (1, 0.00), (1.10, 0.00), (1.20, 0.00), (1.30, 0.00), (1.40, 0.00), (1.50, 0.00), (1.60, $0.00),(1.70,0.00),(1.80,0.00),(1.90,0.00),(2.00,0.00)$

\section{rrsen $=$ GRAPH $(\mathrm{DVS})$}

(0.00, 0.00), (0.1, 0.00), (0.2, 0.00), (0.3, 0.00), (0.4, 0.00), (0.5, 0.00), (0.6, 0.00), (0.7, 0.00), (0.8, 0.00), (0.9, 0.00), (1, 0.00), (1.10, 0.013), (1.20, 0.026), (1.30, 0.04), (1.40, 0.04), (1.50, 0.04), (1.60, 0.04), (1.70, 0.04), (1.80, 0.04), (1.90, 0.04), (2.00, 0.04)

$\mathrm{SLA}=\mathrm{GRAPH}(\mathrm{DVS})$

$(0.00,0.037),(1.00,0.018),(2.00,0.017)$

$\mathrm{VIRn}=\mathrm{GRAPH}(\mathrm{TIME})$

(0.00, 0.00), (10.0, 0.00), (20.0, 0.00), (30.0, 0.002), (40.0, 0.01), (50.0, 0.01), (60.0, 0.01), (70.0, $0.01),(80.0,0.01),(90.0,0.01),(100,0.01),(110,0.01),(120,0.01)$ 


\section{Chapter 9. The RICEPEST and WHEATPEST Models}

This chapter introduces two sibling simulation models that have been developed to simulate yield losses due to rice pests (RICEPEST) and yield losses due to wheat pests (WHEATPEST). Both models have many features in common:

- Objectives of developing RICEPEST and WHEATPEST. Both models were developed in order to simulate yield losses caused by pests (diseases, insects, weeds), individually or in combination, under a range of production situations. This allows (1) a ranking of the pests according to their importance in terms of the yield losses they cause in various production situations and (2) a simulation of the yield gains associated with new technologies, including plant protection methods. The results can provide a formal basis for long term strategies in crop health management, e.g., priority setting for research.

- Conceptual framework. Both models have been developed under the conceptual framework of GENECROP and GENEPEST described in the previous chapters, where relationships between production situations, yield levels, and damage mechanisms are formally captured into simulation models.

- Generic structure of the models. Both RICEPEST and WHEATPEST are built according to the same GENECROP structure, which has been described in Chapter 7 for the simulation of crop growth, and which accounts for damage mechanisms as described under GENEPEST in Chapter 8. In both the rice and the wheat systems, the system considered is $1 \mathrm{~m}^{2}$ of crop in a field, with a time step of one day. Contrary to RICEPEST, WHEATPEST does not include a component for the dynamics of tillers, because the pests addressed in the wheat model do not entail injuries directly affecting tillers.

The purpose of this chapter is to introduce the reader to models that account for multiple injuries. The way these models behave with varying levels of differing injuries is shown later with the simulation models. The details of the models are not discussed, but the reader will find full listings of the programs in Appendices 9.1 and 9.2, as well as references.

\section{Damage mechanisms for a set of pests in rice}

The different rice pests addressed and the mechanisms are summarized in Table 9.1. The details of the inclusion of these damage mechanisms in RICEPEST can be found in Willocquet et al. (1998, 2000, 2002, 2004), and are implemented in the RICEPEST.STMX file. 
Table 9.1. Rice pests addressed, their damage mechanisms, and their effect in RICEPEST

\begin{tabular}{|c|c|c|c|}
\hline Rice pest & $\begin{array}{l}\text { Damage } \\
\text { mechanism }\end{array}$ & Physiological effect & Effect in RICEPEST \\
\hline $\begin{array}{l}\text { Bacterial leaf } \\
\text { blight (BLB) }\end{array}$ & Light stealer & $\begin{array}{l}\text { Reduces the intercepted } \\
\text { radiation }\end{array}$ & Reduces the green LAI \\
\hline Leaf blast (LB) & $\begin{array}{l}\text { Light stealer } \\
\text { Leaf senescence } \\
\text { accelerator } \\
\text { Assimilate sapper }\end{array}$ & $\begin{array}{l}\text { Reduces the intercepted } \\
\text { radiation } \\
\text { Increases leaf senescence } \\
\text { Removes soluble assimilates } \\
\text { from host }\end{array}$ & $\begin{array}{l}\text { Reduces the green LAI (lesion area } \\
+ \text { virtual lesion area) } \\
\text { Reduces the biomass of leaves by } \\
\text { increasing the rate of leaf } \\
\text { senescence } \\
\text { Outflows assimilates from the pool } \\
\text { of assimilates }\end{array}$ \\
\hline $\begin{array}{l}\text { Sheath blight } \\
\text { (SHB) }\end{array}$ & $\begin{array}{l}\text { Light stealer } \\
\text { Leaf senescence } \\
\text { accelerator }\end{array}$ & $\begin{array}{l}\text { Reduces the intercepted } \\
\text { radiation } \\
\text { Increases leaf senescence }\end{array}$ & $\begin{array}{l}\text { Reduces the green LAI } \\
\text { Reduces the biomass of leaves by } \\
\text { increasing the rate of leaf } \\
\text { senescence }\end{array}$ \\
\hline $\begin{array}{l}\text { Brown spot } \\
\text { (BS) }\end{array}$ & Light stealer & $\begin{array}{l}\text { Reduces the intercepted } \\
\text { radiation }\end{array}$ & $\begin{array}{l}\text { Reduces the green LAI (lesion area } \\
+ \text { virtual lesion area) }\end{array}$ \\
\hline $\begin{array}{l}\text { Tungro } \\
\text { (TUNGRO) }\end{array}$ & $\begin{array}{l}\text { Photosynthetic } \\
\text { rate reducer }\end{array}$ & $\begin{array}{l}\text { Disrupts phloem transport } \\
\text { And reduces the rate of } \\
\text { carbon uptake }\end{array}$ & Reduces the RUE \\
\hline $\begin{array}{l}\text { Neck blast } \\
\text { (NB) }\end{array}$ & Tissue consumer & $\begin{array}{l}\text { Disrupts transport of } \\
\text { carbohydrates towards } \\
\text { panicles }\end{array}$ & $\begin{array}{l}\text { Reduces the flow of assimilates } \\
\text { towards panicles }\end{array}$ \\
\hline $\begin{array}{l}\text { Sheath rot } \\
\text { (SHR) }\end{array}$ & Tissue consumer & Disrupts panicle emergence & $\begin{array}{l}\text { Reduces the flow of assimilates } \\
\text { towards panicles }\end{array}$ \\
\hline $\begin{array}{l}\text { White head } \\
\text { (WH) }\end{array}$ & Assimilate sapper & $\begin{array}{l}\text { Disrupts transport of } \\
\text { carbohydrates towards } \\
\text { panicles }\end{array}$ & $\begin{array}{l}\text { Reduces the flow of assimilates } \\
\text { towards panicles }\end{array}$ \\
\hline $\begin{array}{l}\text { Weeds } \\
\text { (WEED) }\end{array}$ & $\begin{array}{l}\text { Photosynthetic } \\
\text { rate reducer }\end{array}$ & $\begin{array}{l}\text { Reduces water and nutrient } \\
\text { supply } \\
\text { Light stealer } \\
\text { Reduction of water, nutrient } \\
\text { and radiation reduces RUE }\end{array}$ & Reduces the RUE \\
\hline $\begin{array}{l}\text { Dead } \\
\text { hearts }(\mathrm{DH})\end{array}$ & Stand reducer & $\begin{array}{l}\text { Reduces the number and } \\
\text { biomass of tillers }\end{array}$ & $\begin{array}{l}\text { Reduces the number of vegetative } \\
\text { tillers }\end{array}$ \\
\hline $\begin{array}{l}\text { Brown plant- } \\
\text { hoppers (BPH) }\end{array}$ & $\begin{array}{l}\text { Assimilate sapper } \\
\text { Leaf senescence } \\
\text { accelerator }\end{array}$ & $\begin{array}{l}\text { Removes soluble assimilates } \\
\text { from host } \\
\text { Increases leaf senescence }\end{array}$ & $\begin{array}{l}\text { Outflows assimilates from the pool } \\
\text { of assimilates } \\
\text { Reduces the biomass of leaves by } \\
\text { increasing the rate of leaf } \\
\text { senescence }\end{array}$ \\
\hline $\begin{array}{l}\text { Defoliators } \\
\text { (DEF) }\end{array}$ & Tissue consumer & Reduces leaf biomass & $\begin{array}{l}\text { Reduces the biomass of leaves by } \\
\text { increasing the rate of leaf } \\
\text { senescence }\end{array}$ \\
\hline
\end{tabular}

Derived from Rabbinge and Vereyken (1980), Rabbinge and Rijsdijk (1981) and Boote et al. (1983). 
Damage mechanisms for a set of pests in wheat

The different wheat pests addressed and the mechanisms are summarized in Table 9.2. The details of the inclusion of these damage mechanisms in WHEATPEST can be found in Willocquet et al. (2008), and are implemented in the WHEATPEST.STMX file.

Table 9.2. Wheat pests addressed, their damage mechanisms, and their effect in WHEATPEST

\begin{tabular}{|c|c|c|c|}
\hline Wheat pest & $\begin{array}{l}\text { Damage } \\
\text { mechanism }^{\text {a }}\end{array}$ & $\begin{array}{l}\text { Physiological } \\
\text { effect }\end{array}$ & Effect in WHEATPEST \\
\hline $\begin{array}{l}\text { Powdery mildew } \\
\text { (PM) }\end{array}$ & Light stealer & $\begin{array}{l}\text { Reduces the } \\
\text { intercepted } \\
\text { radiation }\end{array}$ & $\begin{array}{l}\text { Reduces the green LAI (lesion area }+ \\
\text { virtual lesion area) }\end{array}$ \\
\hline Yellow rust (YR) & $\begin{array}{l}\text { Light stealer } \\
\text { Assimilate } \\
\text { sapper }\end{array}$ & $\begin{array}{l}\text { Reduces the } \\
\text { intercepted } \\
\text { radiation } \\
\text { Removes soluble } \\
\text { assimilates from } \\
\text { host }\end{array}$ & $\begin{array}{l}\text { Reduces the green LAI (lesion area }+ \\
\text { virtual lesion area) } \\
\text { Outflows assimilates from the pool of } \\
\text { assimilates }\end{array}$ \\
\hline Brown rust (BR) & $\begin{array}{l}\text { Light stealer } \\
\text { Assimilate } \\
\text { sapper }\end{array}$ & $\begin{array}{l}\text { Reduces the } \\
\text { intercepted } \\
\text { radiation } \\
\text { Removes soluble } \\
\text { assimilates from } \\
\text { host }\end{array}$ & $\begin{array}{l}\text { Reduces the green LAI } \\
\text { Outflows assimilates from the pool of } \\
\text { assimilates }\end{array}$ \\
\hline $\begin{array}{l}\text { Septoria nodorum } \\
\text { Blotch (SNB) }\end{array}$ & $\begin{array}{l}\text { Light stealer } \\
\text { Assimilate } \\
\text { sapper }\end{array}$ & $\begin{array}{l}\text { Reduces the } \\
\text { intercepted } \\
\text { radiation } \\
\text { Removes soluble } \\
\text { assimilates from } \\
\text { host }\end{array}$ & $\begin{array}{l}\text { Reduces the green LAI } \\
\text { Outflows assimilates from the pool of } \\
\text { assimilates }\end{array}$ \\
\hline $\begin{array}{l}\text { Septoria tritici } \\
\text { Blotch (STB) }\end{array}$ & $\begin{array}{l}\text { Light stealer } \\
\text { Assimilate } \\
\text { sapper }\end{array}$ & $\begin{array}{l}\text { Reduces the } \\
\text { intercepted } \\
\text { radiation } \\
\text { Removes soluble } \\
\text { assimilates from } \\
\text { host }\end{array}$ & $\begin{array}{l}\text { Reduces the green LAI (lesion area }+ \\
\text { virtual lesion area) } \\
\text { Outflows assimilates from the pool of } \\
\text { assimilates }\end{array}$ \\
\hline Take all (TAK) & $\begin{array}{l}\text { Photosynthetic } \\
\text { rate reducer }\end{array}$ & $\begin{array}{l}\text { Disrupts nitrogen } \\
\text { and water uptake }\end{array}$ & Reduces the RUE \\
\hline Eyespot (EYS) & $\begin{array}{l}\text { Photosynthetic } \\
\text { rate reducer }\end{array}$ & $\begin{array}{l}\text { Disrupts nitrogen } \\
\text { and water uptake }\end{array}$ & Reduces the RUE \\
\hline $\begin{array}{l}\text { Sharp eyespot } \\
\text { (SHY) }\end{array}$ & $\begin{array}{l}\text { Photosynthetic } \\
\text { rate reducer }\end{array}$ & $\begin{array}{l}\text { Disrupts nitrogen } \\
\text { and water uptake }\end{array}$ & Reduces the RUE \\
\hline $\begin{array}{l}\text { Fusarium stem rot } \\
\text { (FST) }\end{array}$ & $\begin{array}{l}\text { Photosynthetic } \\
\text { rate reducer }\end{array}$ & $\begin{array}{l}\text { Disrupts nitrogen } \\
\text { and water uptake }\end{array}$ & Reduces the RUE \\
\hline BYDV & $\begin{array}{l}\text { Photosynthetic } \\
\text { rate reducer }\end{array}$ & $\begin{array}{l}\text { Disrupts phloem } \\
\text { transport } \\
\text { Reduces the rate of } \\
\text { carbon uptake }\end{array}$ & Reduces the RUE \\
\hline
\end{tabular}




\begin{tabular}{llll}
\hline $\begin{array}{l}\text { Fusarium Head } \\
\text { Blight (FHB) }\end{array}$ & $\begin{array}{l}\text { Tissue } \\
\text { consumer }\end{array}$ & $\begin{array}{l}\text { Disrupts transport } \\
\text { of carbohydrates } \\
\text { towards ears. }\end{array}$ & $\begin{array}{l}\text { Reduces the flow of assimilates } \\
\text { towards ears }\end{array}$ \\
\hline Weeds (WEED) & $\begin{array}{l}\text { Photosynthetic } \\
\text { rate reducer }\end{array}$ & $\begin{array}{l}\text { Reduces water and } \\
\text { nutrient supply } \\
\text { Light stealer } \\
\text { Reduction of water, } \\
\text { nutrient and } \\
\text { radiation reduces } \\
\text { RUE }\end{array}$ & Reduces the RUE \\
& & $\begin{array}{l}\text { Removes soluble } \\
\text { assimilates from } \\
\text { host }\end{array}$ & $\begin{array}{l}\text { Outflows assimilates from the pool of } \\
\text { assimilates } \\
\text { Aphids (APH) }\end{array}$ \\
& $\begin{array}{l}\text { Assimilate } \\
\text { sapper } \\
\text { Photosynthetic } \\
\text { rate reducer }\end{array}$ & Reduces the RUE & Reduces the RUE \\
\hline
\end{tabular}

${ }^{a}$ Derived from Rabbinge \& Vereyken (1980), Rabbinge \& Rijsdijk (1981) and Boote et al. (1983).

${ }^{\mathrm{b}}$ Production of toxins not included.

Weather and injury drivers in RICEPEST and WHEATPEST

Weather

RICEPEST uses constant daily minimum temperature, maximum temperature and radiation of $24^{\circ} \mathrm{C}, 30^{\circ} \mathrm{C}$, and $17 \mathrm{MJ} / \mathrm{m}^{2}$, respectively. These are within the range of weather values during the rainy season in tropical Asia.

For WHEATPEST, monthly averages of daily temperature and radiation, computed from weather in Wageningen (The Netherlands) during 1951-1980 (Spitters et al., 1989), are interpolated in order to generate daily temperature and radiation.

\section{Injury drivers}

Injuries were not entered in the models as random variables; rather, patterns of injuries are used, which correspond to specific production situations for both rice in tropical Asia (Savary et al., 2000; 2006), and wheat in Western Europe (Polley and Thomas, 1991; Daamen, 1990; Daamen and Stol, 1990, 1992, 1994; Daamen et al., 1989, 1991, 1992). This linkage between production situations and patterns of injuries has been shown to be both reliable and dynamic as production situations evolve (Savary et al. 2006). In turn, the intrinsic rate of (attainable) growth was made dependent on production situations. This was, again, made possible through careful field surveys where yields were measured, experiments, data published in the literature, and a combination of these sources. Thus, the injury drivers actually represent a linkage between production situations - attainable yield - intrinsic crop growth - injury patterns. 
Simulations with RICEPEST and WHEATPEST

The STELLA models RICEPEST.STMX and WHEATPEST.STMX will allow you, for rice and wheat, respectively, to:

- explore the model structure and equations,

- explore the model inputs for attainable growth

- explore the model inputs for actual growth, i.e. the driving functions of the different pests included

- run the model with varying levels of pest inputs, which will allow you to explore:

0 the effects of individual injuries on crop growth and yield

0 the effects of combined injuries on crop growth and yield

\section{Summary}

This chapter describes:

- A formal modeling structure which captures the linkages between production situations attainable yield - intrinsic crop growth - injury patterns.

- The framework and objectives under which RICEPEST and WHEATPEST have been developed.

- The damage mechanisms associated with rice and wheat pests and how the corresponding (multiple) injuries are captured into RICEPEST and WHEATPEST.

- Includes the STELLA files, which can be used to explore the models structures, and the effect of injuries, individually or in combination, on the simulated dynamics of rice and wheat crop growth.

\section{References}

Boote, K. J., Jones, J. W., Mishoe, J. W., and Berger, R. D. 1983. Coupling pests to crop growth simulators to predict yield reductions. Phytopathology 73:1581-1587.

Daamen, R. A. 1990. Surveys of cereal diseases and pests in the Netherlands. 1. Weather and winter wheat cropping during 1974-1986. Neth. J. Plant Pathol. 96:227-236.

Daamen, R. A., and Stol, W. 1990. Surveys of cereal diseases and pests in the Netherlands. 2. Stembase diseases of winter wheat. Neth. J. Plant Pathol. 96:251-260. 
Daamen, R. A., and Stol, W, 1992. Surveys of cereal diseases and pests in the Netherlands. 5. Occurrence of Septoria spp. in winter wheat. Neth. J. Plant Pathol. 98:369-376.

Daamen, R. A., and Stol, W., 1994. Surveys of cereal diseases and pests in the Netherlands. 6. Occurrence of insect pests in winter wheat. Neth. J. Plant Pathol. 99 (Suppl. 3):51-56.

Daamen, R. A.,Wijnands, F. G., and Vliet, G., van der. 1989. Epidemics of diseases and pests of winter wheat at different levels of agrochemical input. A study on the possibilities for designing an integrated cropping system. J. Phytopathol. 125:305-319.

Daamen, R. A., Langerak, C. J., and Stol, W. 1991. Surveys of cereal diseases and pests in the Netherlands. 3. Monographella nivalis and Fusarium spp. In winter wheat fields and seed lots. Neth. J. Plant Pathol. 97:105-114.

Daamen, R. A., Stubbs, R. W., and Stol, W. 1992. Surveys of cereal diseases and pests in the Netherlands. 4. Occurrence of powdery mildew and rusts in winter wheat. Neth. J. Plant Pathol. 98:301-312.

Johnson, K. B. 1992. Evaluation of a mechanistic model that describes potato crop losses caused by multiple pests. Phytopathology 82:363-369.

Polley, R. W., and Thomas, M. R. 1991. Surveys of diseases of winter wheat in England and Wales, 1976-1988. Ann. Appl. Biol. 119:1-20.

Rabbinge, R., and Rijsdijk, P. H. 1981. Disease and crop physiology: a modeler's point of view. Pages 201-220 in: Effects of Disease on the Physiology of the Growing Plants. P. G. Ayres, ed. Cambridge Univ. Press, Cambridge, UK.

Rabbinge, R., and Vereyken, P. H. 1980. The effects of diseases or pests upon host. Z. Pflanzenk. Pflanzensch. 87:409-422.

Savary, S., Willocquet, L., Elazegui, F. A., Teng, P. S., Du, P. V., Zhu, D., Tang, Q., Lin, X., Singh, H. M., and Srivastava, R. K. 2000. Rice pest constraints in tropical Asia: characterization of injury profiles in relation to production situations. Plant Dis. 84:341-356.

Savary, S., Teng, P. S.,Willocquet, L., and Nutter, Jr., F. W. 2006. Quantification and modeling of crop losses: a review of purposes. Annu. Rev. Phytopathol. 44:89-112.

Spitters, C. J. T., van Keulen, H., and van Kraalingen, D. W. G. 1989. A simple and universal crop growth simulator: SUCROS87. Pages 147-181 in: Simulation and Systems Management in Crop Protection. R. Rabbinge, S. A. Ward and H. H. van Laar, eds. Pudoc, Wageningen, The Netherlands.. 
Willocquet, L., Savary, S., Fernandez, L., Elazegui, F. A., and Teng, P. S. 1998. Simulation of yield losses caused by rice diseases, insects, and weeds in tropical Asia. IRRI Discussion Paper Series no 34. IRRI, Los Baños, Philippines.

Willocquet, L., Savary, S., Fernandez, L., Elazegui, F. A., and Teng, P. S. 2000. Development and evaluation of a multiple-pest, production situation specific, simulation model of rice yield losses in tropical Asia. Ecol. Model. 131:133-159.

Willocquet, L., Savary, S., Fernandez, L., Elazegui, F. A., Castilla, N., Zhu, D., Tang, Q., Huang, S., Lin, X., Singh, H. M., and Srivastsava, R. K. 2002. Structure and validation of RICEPEST, a production situation-driven, crop growth model simulating rice yield response to multiple pest injuries for tropical Asia. Ecol. Model. 153:247-268.

Willocquet, L., Elazegui, F. A., Castilla, N., Fernandez, L., Fischer, K. S., Peng, S., Teng, P. S., Srivastava, R. K., Singh, H. M., Zhu, D., and Savary, S. 2004. Research priorities for rice pest management in tropical Asia: a simulation analysis of yield losses and management efficiencies. Phytopathology 94:672-682.

Willocquet, L., Aubertot, J. N., Lebard, S., Robert, C., Lannou, C., and Savary, S. 2008. Simulating multiple pest damage in varying winter wheat production situations. Field Crops Res. 107:12-28.

Appendix 9.1. Program listing of RICEPEST

LeafW $(\mathrm{t})=\operatorname{LeafW}(\mathrm{t}-\mathrm{dt})+($ PartL $-\mathrm{RSenL}) * \mathrm{dt}$

INIT LeafW $=10$

INFLOWS:

PartL $=$ CPL $*$ (Pool-rdiv)

OUTFLOWS:

$\mathrm{RSenL}=((\operatorname{rrsen}+(\operatorname{SenSHB} * \mathrm{SHB})+(\operatorname{SenLB} * \mathrm{LB})+\mathrm{DEF}) * \mathrm{LeafW})+\mathrm{RDHL}+(\operatorname{senBPH} * \mathrm{rdBPH})$

$\operatorname{maxst}(\mathrm{t})=\operatorname{maxst}(\mathrm{t}-\mathrm{dt})+(\operatorname{partScopy}) * \mathrm{dt}$

INIT maxst $=6$

INFLOWS:

partScopy $=$ PartS

$\operatorname{PanW}(\mathrm{t})=\operatorname{PanW}(\mathrm{t}-\mathrm{dt})+(\operatorname{PartP}+\mathrm{RTransloc}-$ inj_pan $) * d t$

INIT PanW $=0$

INFLOWS:

PartP $=\mathrm{CPP} *($ Pool-rdiv $)$ 
RTransloc $=\operatorname{IF}($ DVS $>1)$ then DDIST else 0

OUTFLOWS:

inj_pan $=($ PartP+RTransloc $) *(\mathrm{NB}+\mathrm{SHR}+\mathrm{WH}-(\mathrm{NB} * \mathrm{SHR})-(\mathrm{NB} * \mathrm{WH})-(\mathrm{SHR} * \mathrm{WH})-(\mathrm{NB} * \mathrm{SHR} * \mathrm{WH}))$

$\operatorname{Pool}(\mathrm{t})=\operatorname{Pool}(\mathrm{t}-\mathrm{dt})+($ RGrowth - PartS - PartL - PartP - PartR - rdiv $) * d t$

INIT Pool $=0$

INFLOWS:

RGrowth $=$ RAD*RUE*(1-EXP(-k*LAI) $) *(1-(1-\exp (-0.003 *$ WEED $))) *(1-(0.7 *$ Virdis $)) *$ pRUE

OUTFLOWS:

PartS $=$ CPS $*($ Pool-rdiv $)$

PartL $=$ CPL $*$ (Pool-rdiv)

PartP $=\mathrm{CPP} *($ Pool-rdiv $)$

PartR $=\mathrm{CPR} *($ Pool-rdiv $)$

$\operatorname{rdiv}=($ rsuck $* \mathrm{BPH})+(\mathrm{LB} * \mathrm{LAI} * \mathrm{LBspoDW})$

$\operatorname{REPTIL}(\mathrm{t})=\operatorname{REPTIL}(\mathrm{t}-\mathrm{dt})+($ Rmat $-\mathrm{Rmortr}) * \mathrm{dt}$

INIT REPTIL $=0$

INFLOWS:

Rmat $=$ if DVS $<0.8$ or DVS $>1$ then 0 else if VTIL $<$ FST $*$ Totil then 0 else RRMAT*VTIL

OUTFLOWS:

Rmortr $=$ rrmort $*$ REPTIL

$\operatorname{RootW}(\mathrm{t})=\operatorname{Root} W(\mathrm{t}-\mathrm{dt})+(\operatorname{PartR}) * \mathrm{dt}$

INIT RootW $=5$

INFLOWS:

PartR $=$ CPR $*$ (Pool-rdiv)

$\operatorname{SDEFn}(\mathrm{t})=\operatorname{SDEFn}(\mathrm{t}-\mathrm{dt})+(\operatorname{RDEF}) * d \mathrm{t}$

INIT SDEFn $=0$

INFLOWS:

$\mathrm{RDEF}=\mathrm{DEF}$

$\operatorname{SDHn}(\mathrm{t})=\operatorname{SDHn}(\mathrm{t}-\mathrm{dt})+(\mathrm{RDH}) * \mathrm{dt}$

INIT SDHn $=0$

INFLOWS:

$\mathrm{RDH}=\mathrm{DHn}$

$\operatorname{STEMP}(\mathrm{t})=\operatorname{STEMP}(\mathrm{t}-\mathrm{dt})+($ Dtemp $) * d t$

INIT STEMP $=320$ 
INFLOWS:

Dtemp $=(($ TMAX + TMIN $) / 2)-$ TBASE

StemW $(\mathrm{t})=\operatorname{StemW}(\mathrm{t}-\mathrm{dt})+($ PartS - RTransloc - RsenST $) * d t$

INIT StemW $=6$

INFLOWS:

PartS $=$ CPS $*$ (Pool-rdiv)

OUTFLOWS:

RTransloc $=\operatorname{IF}($ DVS $>1)$ then DDIST else 0

RsenST $=$ RDHST

$\operatorname{VTIL}(\mathrm{t})=\operatorname{VTIL}(\mathrm{t}-\mathrm{dt})+($ Rtil - Rmat $-\mathrm{Rmrtv}) * \mathrm{dt}$

INIT VTIL $=250$

INFLOWS:

Rtil $=$ PartLS $*$ STW $*(1-($ VTIL/Maxtil $)) * D V E$

OUTFLOWS:

Rmat $=$ if DVS $<0.8$ or DVS $>1$ then 0 else if VTIL $<$ FST $*$ Totil then 0 else RRMAT*VTIL

Rmrtv $=($ rrmort $*$ VTIL $)+\mathrm{DH}$

BetaBS $=6.3$

BetaLB $=3$

$\mathrm{BLB}=\mathrm{pBLB} * \mathrm{BLBn}$

$\mathrm{BLBn}=\mathrm{GRAPH}(\mathrm{TIME})$

$(0.00,0.00),(24.0,0.00),(48.0,0.00),(72.0,0.005),(96.0,0.01),(120,0.00)$

$\mathrm{BPH}=\mathrm{pBPH}{ }^{*} \mathrm{BPHn}$

$\mathrm{BPHn}=\mathrm{GRAPH}(\mathrm{TIME})$

(0.00, 0.00), (10.0, 0.00), (20.0, 0.00), (30.0, 0.00), (40.0, 60.0), (50.0, 125), (60.0, 190), (70.0, 250), $(80.0,250),(90.0,145),(100,65.0),(110,0.00),(120,0.00)$

$\mathrm{BS}=\mathrm{pBS} * \mathrm{BSn}$

$\mathrm{BSn}=\mathrm{GRAPH}(\mathrm{TIME})$

$(0.00,0.00),(24.0,0.00),(48.0,0.00),(72.0,0.005),(96.0,0.01),(120,0.01)$

$\mathrm{CPL}=\mathrm{CPPL} *(1-\mathrm{CPR})$

$\mathrm{CPP}=\mathrm{CPPP} *(1-\mathrm{CPR})$

$\mathrm{CPPL}=\mathrm{GRAPH}(\mathrm{DVS})$ 
(0.00, 0.55), (0.1, 0.536), (0.2, 0.521), (0.3, 0.507), (0.4, 0.493), (0.5, 0.479), (0.6, 0.464), (0.7, 0.45),

$(0.8,0.3),(0.9,0.15),(1,0.00),(1.10,0.00),(1.20,0.00),(1.30,0.00),(1.40,0.00),(1.50,0.00)$, $(1.60,0.00),(1.70,0.00),(1.80,0.00),(1.90,0.00),(2.00,0.00)$

\section{$\mathrm{CPPP}=\mathrm{GRAPH}(\mathrm{DVS})$}

(0.00, 0.00), (0.05, 0.00), (0.1, 0.00), (0.15, 0.00), (0.2, 0.00), (0.25, 0.00), (0.3, 0.00), (0.35, 0.00),

$(0.4,0.00),(0.45,0.00),(0.5,0.00),(0.55,0.00),(0.6,0.00),(0.65,0.00),(0.7,0.00),(0.75,0.00)$, $(0.8,0.143),(0.85,0.286),(0.9,0.429),(0.95,0.571),(1.00,0.714),(1.05,0.857),(1.10,1.00)$, $(1.15,1.00),(1.20,1.00),(1.25,1.00),(1.30,1.00),(1.35,1.00),(1.40,1.00),(1.45,1.00),(1.50$, 1.00), (1.55, 1.00), (1.60, 1.00), (1.65, 1.00), (1.70, 1.00), (1.75, 1.00), (1.80, 1.00), (1.85, 1.00), $(1.90,1.00),(1.95,1.00),(2.00,1.00)$

\section{$\mathrm{CPR}=\mathrm{GRAPH}(\mathrm{DVS})$}

(0.00, 0.3), (0.1, 0.263), (0.2, 0.225), (0.3, 0.188), (0.4, 0.15), (0.5, 0.112), (0.6, 0.075), (0.7, 0.038),

$(0.8,0.00),(0.9,0.00),(1,0.00),(1.10,0.00),(1.20,0.00),(1.30,0.00),(1.40,0.00),(1.50,0.00)$,

$(1.60,0.00),(1.70,0.00),(1.80,0.00),(1.90,0.00),(2.00,0.00)$

\section{$\mathrm{CPS}=(1-\mathrm{CPL}-\mathrm{CPP}) *(1-\mathrm{CPR})$}

cumul\%DH $=100 * \mathrm{SDH} / \mathrm{Totil}$

$\mathrm{DACE}=\mathrm{TIME}+14$

$\mathrm{dBPHDWn}=$ GRAPH$(\mathrm{TIME})$

(0.00, 0.00), (1.00, 0.00), (2.00, 0.00), (3.00, 0.00), (4.00,0.00), (5.00, 0.00), (6.00, 0.00), (7.00, 0.00), (8.00, 0.00), (9.00, 0.00), (10.0, 0.00), (11.0, 0.00), (12.0, 0.00), (13.0, 0.00), (14.0, 0.00), (15.0, 0.00), (16.0, 0.00), (17.0, 0.00), (18.0, 0.00), (19.0, 0.00), (20.0, 0.00), (21.0, 0.00), (22.0, 0.00), (23.0, 0.00), (24.0, 0.00), (25.0, 0.00), (26.0, 0.00), (27.0, 0.00), (28.0, 0.00), (29.0, 0.00), (30.0, 0.0125), (31.0, 0.0125), (32.0, 0.0125), (33.0, 0.0125), (34.0, 0.0125), (35.0, 0.0125), (36.0, 0.0125), (37.0, 0.0125), (38.0, 0.0125), (39.0, 0.0125), (40.0, 0.0125), (41.0, 0.0125), (42.0, 0.0125), (43.0, 0.0125), (44.0, 0.0125), (45.0, 0.0125), (46.0, 0.0125), (47.0, 0.0125), (48.0, 0.0125), (49.0, 0.0125), (50.0, 0.0125), (51.0, 0.0125), (52.0, 0.0125), (53.0, 0.0125), (54.0, 0.0125), (55.0, 0.0125), (56.0, 0.0125), (57.0, 0.0125), (58.0, 0.0125), (59.0, 0.0125), (60.0, 0.0125), (61.0, 0.0125), (62.0, 0.0125), (63.0, 0.0125), (64.0, 0.0125), (65.0, 0.0125), (66.0, 0.0125), (67.0, 0.0125), (68.0, 0.0125), (69.0, 0.0125), (70.0, 0.0125), (71.0, 0.00), (72.0, 0.00), (73.0, 0.00), (74.0, 0.00), (75.0, 0.00), (76.0, 0.00), (77.0, 0.00), (78.0, 0.00), (79.0, 0.00), (80.0, 0.00), (81.0, 0.00), (82.0, 0.00), (83.0, 0.00), (84.0, 0.00), (85.0, 0.00), (86.0, 0.00), (87.0, 0.00), (88.0, 0.00), (89.0, 0.00), (90.0, 0.00), (91.0, 0.00), (92.0, 0.00), (93.0, 0.00), (94.0, 0.00), (95.0, 0.00), (96.0, 0.00), (97.0, 0.00), (98.0, 0.00), (99.0, 0.00), (100, 0.00), (101, 0.00), (102, 0.00), 
(103, 0.00), (104, 0.00), (105, 0.00), (106, 0.00), (107, 0.00), (108, 0.00), (109, 0.00), (110, 0.00), $(111,0.00),(112,0.00),(113,0.00),(114,0.00),(115,0.00),(116,0.00),(117,0.00),(118,0.00)$, $(119,0.00),(120,0.00)$

ddist $=0.0067 * \operatorname{maxst}$

$\mathrm{DEF}=\mathrm{pDEF} * \mathrm{DEFn}$

$\mathrm{DEFn}=\mathrm{GRAPH}(\mathrm{TIME})$

(0.00, 0.00), (10.0, 0.00), (20.0, 0.00), (30.0, 0.0003), (40.0, 0.0005), (50.0, 0.0002), (60.0, 0.00), (70.0, 0.00), (80.0, 0.00), (90.0, 0.00), (100, 0.00), (110,0.00), (120,0.00)

$\mathrm{DH}=\mathrm{pDH} * \mathrm{DHn}$

$\mathrm{DHn}=\mathrm{GRAPH}(\mathrm{TIME})$

(0.00, 0.00), (10.0, 0.00), (20.0, 0.00), (30.0, 0.00), (40.0, 0.5), (50.0, 0.00), (60.0, 0.00), (70.0, 0.00), $(80.0,0.00),(90.0,0.00),(100,0.00),(110,0.00),(120,0.00)$

$\mathrm{DVE}=\mathrm{GRAPH}(\mathrm{DVS})$

$(0.00,1.00),(0.4,1.00),(0.8,0.00),(1.20,0.00),(1.60,0.00),(2.00,0.00)$

DVS $=$ if stemp $<$ TFLOW then STEMP/TFLOW ELSE 1+((STEMP-TFLOW)/(TMAT-TFLOW $))$

DWBPH $=0.001$

$\mathrm{FST}=0.05$

grain_yield $=0.85 *$ PanW

$\mathrm{k}=0.6$

$\mathrm{LAI}=\mathrm{LeafW}^{*} \mathrm{SLA} *(1-\mathrm{BLB}) *\left((1-\mathrm{BS})^{\wedge} \mathrm{BetaBS}\right) *(1-\mathrm{SHB}) *\left((1-\mathrm{LB})^{\wedge} \mathrm{BetaLB}\right)$

$\mathrm{LB}=\mathrm{pLB} * \mathrm{LBn}$

$\mathrm{LBn}=\mathrm{GRAPH}(\mathrm{TIME})$

(0.00, 0.00), (5.00, 0.00), (10.0, 0.00), (15.0, 0.002), (20.0, 0.004), (25.0, 0.007), (30.0, 0.01), (35.0, 0.005), (40.0, 0.00), (45.0, 0.00), (50.0,0.00), (55.0, 0.00), (60.0, 0.00), (65.0, 0.00), (70.0, 0.00), (75.0, 0.00), (80.0, 0.00), (85.0, 0.00), (90.0, 0.00), (95.0, 0.00), (100, 0.00), (105, 0.00), (110, $0.00),(115,0.00),(120,0.00)$

\section{LBspoDW = GRAPH(TIME)}

(0.00, 0.00), (1.00, 0.00), (2.00, 0.00), (3.00, 0.00), (4.00, 0.00), (5.00, 0.00), (6.00, 0.00), (7.00, 0.00), (8.00, 0.00), (9.00, 0.00), (10.0, 20.0), (11.0, 20.0), (12.0, 20.0), (13.0, 20.0), (14.0, 20.0), (15.0, 20.0), (16.0, 20.0), (17.0, 20.0), (18.0, 20.0), (19.0, 20.0), (20.0, 20.0), (21.0, 20.0), (22.0, 20.0), (23.0, 20.0), (24.0, 20.0), (25.0, 0.00), (26.0, 0.00), (27.0, 0.00), (28.0, 0.00), (29.0, 0.00), (30.0, 0.00), (31.0, 0.00), (32.0, 0.00), (33.0, 0.00), (34.0, 0.00), (35.0, 0.00), (36.0, 0.00), (37.0, 0.00), $(38.0,0.00),(39.0,0.00),(40.0,0.00),(41.0,0.00),(42.0,0.00),(43.0,0.00),(44.0,0.00),(45.0$, 
0.00), (46.0, 0.00), (47.0, 0.00), (48.0, 0.00), (49.0, 0.00), (50.0, 0.00), (51.0, 0.00), (52.0, 0.00), (53.0, 0.00), (54.0, 0.00), (55.0, 0.00), (56.0, 0.00), (57.0, 0.00), (58.0, 0.00), (59.0, 0.00), (60.0, $0.00),(61.0,0.00),(62.0,0.00),(63.0,0.00),(64.0,0.00),(65.0,0.00),(66.0,0.00),(67.0,0.00)$, (68.0, 0.00), (69.0, 0.00), (70.0, 0.00), (71.0, 0.00), (72.0, 0.00), (73.0, 0.00), (74.0, 0.00), (75.0, 0.00), (76.0, 0.00), (77.0, 0.00), (78.0, 0.00), (79.0, 0.00), (80.0, 0.00), (81.0, 0.00), (82.0, 0.00), (83.0, 0.00), (84.0, 0.00), (85.0, 0.00), (86.0, 0.00), (87.0, 0.00), (88.0, 0.00), (89.0, 0.00), (90.0, 0.00), (91.0, 0.00), (92.0, 0.00), (93.0, 0.00), (94.0, 0.00), (95.0, 0.00), (96.0, 0.00), (97.0, 0.00), (98.0, 0.00), (99.0, 0.00), (100, 0.00), (101, 0.00), (102, 0.00), (103, 0.00), (104, 0.00), (105, 0.00), (106, 0.00), (107, 0.00), (108, 0.00), (109, 0.00), (110, 0.00), (111, 0.00), (112, 0.00), (113, $0.00),(114,0.00),(115,0.00),(116,0.00),(117,0.00),(118,0.00),(119,0.00),(120,0.00)$

\section{LWT $=$ LeafW/VTIL}

Maxtil $=900$

$\mathrm{NB}=\mathrm{pNB} * \mathrm{NBn}$

$\mathrm{NBn}=\mathrm{GRAPH}(\mathrm{TIME})$

$(0.00,0.01),(120,0.01)$

PartLS $=$ PartL+PartS

$\mathrm{pBLB}=0$

$\mathrm{pBPH}=0$

$\mathrm{pBS}=0$

$\mathrm{pDEF}=0$

$\mathrm{pDH}=0$

$\mathrm{pLB}=0$

$\mathrm{pNB}=0$

$\mathrm{pRUE}=1$

$\mathrm{pSHB}=0$

$\mathrm{pSHR}=0$

$\mathrm{pVD}=0$

$\mathrm{pWEED}=0$

$\mathrm{pWH}=0$

$\mathrm{RAD}=17$

$\mathrm{rdBPH}=$ if $\mathrm{DVS}<1$ then 0 else $\mathrm{pBPH} * \mathrm{dBPHDWn}$

$\mathrm{RDHL}=\mathrm{LWT} * \mathrm{DH}$

$\mathrm{RDHST}=\mathrm{STWT} * \mathrm{DH}$ 
RRMAT $=0.3$

rrmort $=$ GRAPH $($ DVS $)$

(0.00, 0.00), (0.1, 0.00), (0.2, 0.00), (0.3, 0.00), (0.4, 0.00), (0.5, 0.02), (0.6, 0.02), (0.7, 0.02), (0.8, $0.02),(0.9,0.02),(1,0.00),(1.10,0.00),(1.20,0.00),(1.30,0.00),(1.40,0.00),(1.50,0.00)$, $(1.60,0.00),(1.70,0.00),(1.80,0.00),(1.90,0.00),(2.00,0.00)$

rrsen $=$ GRAPH $(\mathrm{DVS})$

(0.00, 0.00), (0.1, 0.00), (0.2, 0.00), (0.3, 0.00), (0.4, 0.00), (0.5, 0.00), (0.6, 0.00), (0.7, 0.00), (0.8, $0.00),(0.9,0.00),(1,0.00),(1.10,0.013),(1.20,0.026),(1.30,0.04),(1.40,0.04),(1.50,0.04)$, $(1.60,0.04),(1.70,0.04),(1.80,0.04),(1.90,0.04),(2.00,0.04)$

rsuck $=0.002$

RUE $=$ GRAPH $(D V S)$

(0.00, 1.20), (0.1, 1.20), (0.2, 1.20), (0.3, 1.20), (0.4, 1.20), (0.5, 1.20), (0.6, 1.20), (0.7, 1.20), (0.8, $1.20),(0.9,1.20),(1,1.15),(1.10,1.10),(1.20,1.10),(1.30,1.10),(1.40,1.10),(1.50,1.10)$, $(1.60,1.10),(1.70,1.10),(1.80,1.10),(1.90,1.10),(2.00,1.10)$

$\operatorname{senBPH}=6$

SenLB $=0.0378$

SenSHB $=0.076$

$\mathrm{SHB}=\mathrm{pSHB} * \mathrm{SHBn}$

$\mathrm{SHBn}=\mathrm{GRAPH}(\mathrm{TIME})$

$(0.00,0.00),(10.0,0.00),(20.0,0.00),(30.0,0.00),(40.0,0.0023),(50.0,0.0033),(60.0,0.0066)$, (70.0, 0.01), (80.0, 0.0088), (90.0, 0.0077), (100, 0.0066), (110, 0.0066), (120, 0.0066)

SHR $=$ pSHR $*$ SHRn

SHRn $=$ GRAPH $($ TIME $)$

$(0.00,0.01),(120,0.01)$

SLA $=$ GRAPH(DVS)

(0.00, 0.037), (1.00, 0.018), (2.00, 0.017)

$\mathrm{STW}=20$

STWT $=$ StemW $/$ VTIL

TBASE $=8$

TFLOW $=1450$

TMAT $=2030$

TMAX $=30$

$\mathrm{TMIN}=24$ 
Totil $=$ VTIL + REPTIL

Virdis $=\mathrm{pVD} *$ Virdisn

Virdisn = GRAPH $($ TIME $)$

(0.00, 0.00), (10.5, 0.00), (21.0, 0.00), (31.5, 0.005), (42.0, 0.01), (52.5, 0.01), (63.0, 0.01), (73.5, $0.01),(84.0,0.01),(94.5,0.01),(105,0.01)$

$\mathrm{WEED}=\mathrm{pWEED} * \mathrm{WEEDn}$

WEEDn $=$ GRAPH $($ TIME $)$

(0.00, 0.00), (10.0, 1.00), (20.0, 2.00), (30.0, 3.00), (40.0, 4.00), (50.0, 5.00), (60.0, 6.00), (70.0, 7.00), $(80.0,8.00),(90.0,9.00),(100,10.0),(110,10.0),(120,10.0)$

$\mathrm{WH}=\mathrm{pWH}{ }^{*} \mathrm{WHn}$

$\mathrm{WHn}=\mathrm{GRAPH}(\mathrm{TIME})$

$(0.00,0.01),(120,0.01)$

Appendix 9.2. Program listing of WHEATPEST

$\operatorname{EarB}(\mathrm{t})=\operatorname{EarB}(\mathrm{t}-\mathrm{dt})+(\operatorname{PartE}+\mathrm{RTransloc}-$ inj_ear $) * d t$

INIT EarB $=0$

INFLOWS:

PartE $=\mathrm{CPE}^{*}($ Pool-rasdiv $)$

RTransloc $=\operatorname{IF}($ DVS $>1)$ then ddist else 0

OUTFLOWS:

inj_ear $=($ PartE + RTransloc $) *(1.1 *$ FHB $)$

$\operatorname{Honey}(\mathrm{t})=\operatorname{Honey}(\mathrm{t}-\mathrm{dt})+($ rhoney $) * \mathrm{dt}$

INIT Honey $=0$

INFLOWS:

rhoney $=0.35 *$ rsap

$\operatorname{LeafB}(\mathrm{t})=\operatorname{LeafB}(\mathrm{t}-\mathrm{dt})+(\operatorname{PartL}-\mathrm{RSenL}) * \mathrm{dt}$

INIT LeafB $=10$

INFLOWS:

PartL $=$ CPL $*$ (Pool-rasdiv)

OUTFLOWS:

RSenL $=$ rrsen $*$ LeafB

$\operatorname{MaxStemb}(\mathrm{t})=\operatorname{MaxStemb}(\mathrm{t}-\mathrm{dt})+(\operatorname{rmaxstemb}) * \mathrm{dt}$ 
INIT MaxStemb $=6$

INFLOWS:

rmaxstemb $=$ PartS

$\operatorname{Pool}(\mathrm{t})=\operatorname{Pool}(\mathrm{t}-\mathrm{dt})+($ RGrowth - PartS - PartL - PartE - PartR - rasdiv $) * d t$

INIT Pool $=0$

INFLOWS:

RGrowth $\quad=\quad$ RAD*RUE*(1-EXP(-k*LAI))*(1-(0.35*BYDV))*(1-TAK)*(1-(1-exp $(-$ $0.003 * \mathrm{WEED}))) *(\operatorname{MAX}(0,(1-(0.63 * \mathrm{SNB})))) *(\operatorname{MAX}(0,(1-(0.63 * \mathrm{STB})))) *(1-(0.35 * \mathrm{EYS})) *(1-$

$(0.3 * \mathrm{SHY})) *(1-(0.45 * \mathrm{FST})) * \mathrm{rfaph}$

OUTFLOWS:

PartS $=$ CPS $*($ Pool-rasdiv)

PartL $=$ CPL $*$ (Pool-rasdiv)

PartE $=\mathrm{CPE} *($ Pool-rasdiv $)$

PartR $=\mathrm{CPR} *($ Pool-rasdiv $)$

$\operatorname{rasdiv}=(\min (\operatorname{Pool},(4.62 * \mathrm{YR} * \mathrm{LAI})+(4.62 * \mathrm{BR} * \mathrm{LAI})+\mathrm{rsap}))$

$\operatorname{RootB}(\mathrm{t})=\operatorname{RootB}(\mathrm{t}-\mathrm{dt})+(\operatorname{PartR}) * \mathrm{dt}$

INIT RootB $=5$

INFLOWS:

PartR $=\mathrm{CPR} *($ Pool-rasdiv $)$

$\operatorname{StemB}(\mathrm{t})=\operatorname{StemB}(\mathrm{t}-\mathrm{dt})+(\operatorname{PartS}-\mathrm{RTransloc}) * \mathrm{dt}$

INIT StemB $=6$

INFLOWS:

PartS $=$ CPS $*($ Pool-rasdiv $)$

OUTFLOWS:

RTransloc $=\operatorname{IF}($ DVS $>1)$ then ddist else 0

$\operatorname{STEMP}(\mathrm{t})=\operatorname{STEMP}(\mathrm{t}-\mathrm{dt})+($ Dtemp $) * d t$

INIT STEMP $=620$

INFLOWS:

Dtemp $=(($ TMAX + TMIN $) / 2)-$ TBASE

aphfw $=\mathrm{APHN}^{*} \mathrm{sfwaph}^{*}$ multact

$\mathrm{APHN}=\mathrm{APHNn} * \mathrm{pAPHN}$

$\mathrm{APHNn}=\mathrm{GRAPH}(\mathrm{TIME})$ 
$(145,0.00),(155,25.0),(165,50.0),(175,70.0),(185,125),(195,250),(205,0.00),(215,0.00),(225$, $0.00)$

BetaPM $=2.5$

BetaSTB $=1.25$

BetaYR $=1.5$

$\mathrm{BR}=\mathrm{pBR} * \mathrm{BRn}$

$\mathrm{BRn}=\mathrm{GRAPH}(\mathrm{TIME})$

(75.0, 0.00), (100, 0.0004), (125, 0.0008), (150, 0.001), (175, 0.006), (200, 0.01), (225, 0.01)

$\mathrm{BYDV}=\mathrm{pBYDV} * \mathrm{BYDVn}$

BYDVn $=$ GRAPH(TIME)

(75.0, 0.01), (90.0, 0.01), (105, 0.01), (120,0.01), (135, 0.01), (150,0.01), (165, 0.01), (180, 0.01), $(195,0.01),(210,0.01),(225,0.01)$

$\mathrm{CPE}=(1-\mathrm{CPR}) *(1-\mathrm{CpPL}-\mathrm{CPpS})$

$\mathrm{CPL}=\mathrm{CPPL} *(1-\mathrm{CPR})$

CPPL $=$ GRAPH(DVS)

(0.00, 0.65), (0.05, 0.65), (0.1, 0.65), (0.15, 0.67), (0.2, 0.69), (0.25, 0.7), (0.3, 0.66), (0.35, 0.62), (0.4, 0.58), (0.45, 0.54), (0.5, 0.5), (0.55, 0.41), (0.6, 0.32), (0.65, 0.23), (0.7, 0.15), $(0.75,0.12),(0.8$, 0.09), (0.85, 0.06), (0.9, 0.04), (0.95, 0.00), (1.00, 0.00), (1.05, 0.00), (1.10, 0.00), (1.15, 0.00), (1.20, 0.00), (1.25, 0.00), (1.30, 0.00), (1.35, 0.00), (1.40, 0.00), (1.45, 0.00), (1.50, 0.00), (1.55, $0.00),(1.60,0.00),(1.65,0.00),(1.70,0.00),(1.75,0.00),(1.80,0.00),(1.85,0.00),(1.90,0.00)$, $(1.95,0.00),(2.00,0.00)$

\section{CPPS = GRAPH(DVS)}

(0.00, 0.35), (0.05, 0.35), (0.1, 0.35), (0.15, 0.33), (0.2, 0.31), (0.25, 0.3), (0.3, 0.34), (0.35, 0.38), (0.4, 0.42), (0.45, 0.46), (0.5, 0.5), (0.55, 0.59), (0.6, 0.68), (0.65, 0.77), (0.7, 0.85), (0.75, 0.88), (0.8, 0.91), (0.85, 0.94), (0.9, 0.96), (0.95, 1.00), (1.00, 0.5), (1.05, 0.00), (1.10, 0.00), (1.15, 0.00), (1.20, 0.00), (1.25, 0.00), (1.30, 0.00), (1.35, 0.00), (1.40, 0.00), (1.45, 0.00), (1.50, 0.00), (1.55, 0.00), (1.60, 0.00), (1.65, 0.00), (1.70, 0.00), (1.75, 0.00), (1.80, 0.00), (1.85, 0.00), (1.90, 0.00), $(1.95,0.00),(2.00,0.00)$

\section{$\mathrm{CPR}=\mathrm{GRAPH}(\mathrm{DVS})$}

(0.00, 0.5), (0.1, 0.5), (0.2, 0.4), (0.3, 0.3), (0.4, 0.17), (0.5, 0.13), (0.6, 0.1), (0.7, 0.07), (0.8, 0.05), (0.9, 0.03), (1, 0.02), (1.10, 0.01), (1.20, 0.00), (1.30, 0.00), (1.40, 0.00), (1.50, 0.00), (1.60, 0.00), (1.70, 0.00), (1.80, 0.00), (1.90, 0.00), (2.00, 0.00)

$\mathrm{CPS}=\mathrm{CPPS} *(1-\mathrm{CPR})$ 
ddist $=0.0025 *$ MaxStemb

DVS $=$ if stemp $<$ TFLOW then STEMP/TFLOW ELSE $1+(($ STEMP-TFLOW $) /($ TMAT-TFLOW $))$

$\mathrm{EYS}=\mathrm{pEYS} * \mathrm{EYSn}$

$\mathrm{EYSn}=\mathrm{GRAPH}(\mathrm{TIME})$

(75.0, 0.00), (150, 0.00), (225, 0.01)

$\mathrm{FHB}=\mathrm{pFHB} * \mathrm{FHBn}$

$\mathrm{FHBn}=\mathrm{GRAPH}(\mathrm{TIME})$

$(75.0,0.01),(225,0.01)$

$\mathrm{FST}=\mathrm{pFST} * \mathrm{FSTn}$

$\mathrm{FST} n=$ GRAPH$($ TIME $)$

(75.0, 0.00), (150, 0.00), (225, 0.01)

grain_yield $=0.85 *$ EarB

$\mathrm{k}=0.65$

$\mathrm{LAI}=\mathrm{LeafB} * \mathrm{SLA} *\left((1-\mathrm{PM})^{\wedge} \mathrm{BetaPM}\right) *(1-\mathrm{SNB}) *\left((1-\mathrm{STB})^{\wedge} \mathrm{BetaSTB}\right) *\left((1-\mathrm{YR})^{\wedge} \text { BetaYR }\right)^{*}(1-\mathrm{BR})$

multact $=0.001$

$\mathrm{pAPHN}=0$

PartLS $=$ PartL + PartS

$\mathrm{pBR}=0$

$\mathrm{pBYDV}=0$

$\mathrm{pEYS}=0$

$\mathrm{pFHB}=0$

$\mathrm{pFST}=0$

$\mathrm{PM}=\mathrm{pPM} * \mathrm{PMn}$

$\mathrm{PMn}=\mathrm{GRAPH}(\mathrm{TIME})$

(75.0, 0.00), (100, 0.001), (125, 0.002), (150, 0.003), (175, 0.0065), (200, 0.01), (225, 0.01)

$\mathrm{pPM}=0$

$\mathrm{pRUE}=1$

$\mathrm{pSHY}=0$

$\mathrm{pSNB}=0$

$\mathrm{pSTB}=0$

$\mathrm{pTAK}=0$

$\mathrm{pWEED}=0$

$\mathrm{pYR}=0$ 
$\mathrm{RAD}=\mathrm{GRAPH}(\mathrm{TIME})$

(75.0, 7.80), (105, 13.0), (135, 16.3), (165, 17.5), (195, 15.6), (225, 13.8)

$\mathrm{rfaph}=\max (1-($ Honey*0.015),0.8)

rrsap $=$ GRAPH $($ TIME $)$

(75.0, 0.45), (90.0, 0.45), (105, 0.45), (120, 0.45), (135, 0.45), (150,0.45), (165, 0.45), (180, 0.45), $(195,0.32),(210,0.2),(225,0.24)$

rrsen $=$ GRAPH $($ DVS $)$

(0.00, 0.00), (0.2, 0.00), (0.4, 0.00), (0.6, 0.00), (0.8, 0.00), (1.00, 0.00), (1.20, 0.01), (1.40, 0.025), $(1.60,0.04),(1.80,0.1),(2.00,0.1)$

rsap $=\operatorname{rrsap}^{*}$ aphfw

RUE $=$ GRAPH $(D V S * p R U E)$

$(0.1,1.20),(0.3,1.20),(0.5,1.20),(0.7,1.20),(0.9,1.20),(1.10,1.10),(1.30,1.10),(1.50,1.10),(1.70$, $1.10),(1.90,1.10),(2.10,1.10)$

sfwaph $=$ GRAPH(TIME)

(75.0, 0.00), (85.7, 0.00), (96.4, 0.00), (107, 0.05), (118, 0.1), (129, 0.15), (139, 0.2), (150, 0.25), (161, 0.316), (171, 0.316), (182, 0.283), (193, 0.25), (204, 0.33), (214, 0.415), (225, 0.415)

$\mathrm{SHY}=\mathrm{pSHY} * \mathrm{SHYn}$

$\mathrm{SHYn}=$ GRAPH$(\mathrm{TIME})$

$(75.0,0.00),(150,0.00),(225,0.01)$

$\mathrm{SLA}=\mathrm{GRAPH}(\mathrm{DVS})$

$(0.00,0.037),(1.00,0.018),(2.00,0.017)$

$\mathrm{SNB}=\mathrm{pSNB} * \mathrm{SNBn}$

$\mathrm{SNBn}=\mathrm{GRAPH}(\mathrm{TIME})$

(75.0, 0.00), (100, 0.0003), (125, 0.0006), (150, 0.001), (175, 0.0055), (200, 0.01), (225, 0.01)

$\mathrm{STB}=\mathrm{pSTB} * \mathrm{STBn}$

$\mathrm{STBn}=\mathrm{GRAPH}(\mathrm{TIME})$

(75.0, 0.00), (100, 0.0003), (125, 0.0006), (150, 0.001), (175, 0.0055), (200, 0.01), (225, 0.01)

$\mathrm{TAK}=$ pTAK $*$ TAKn

$\mathrm{TAKn}=\mathrm{GRAPH}(\mathrm{TIME})$

(75.0, 0.0005), (100, 0.001), (125, 0.0015), (150, 0.002), (175, 0.006), (200, 0.01), (225, 0.01)

TBASE $=0$

TFLOW $=1600$

$\mathrm{TMAT}=2500$ 
TMAX $=$ GRAPH(TIME)

(75.0, 8.90), (105, 12.4), (135, 17.3), (165, 20.5), (195, 21.4), (225, 21.5)

$\mathrm{TMIN}=$ GRAPH(TIME)

(75.0, 1.20), (105, 3.30), (135, 7.30), (165, 10.3), (195, 12.2), (225, 12.0)

$\mathrm{WEED}=\mathrm{pWEED} * \mathrm{WEEDn}$

WEEDn = GRAPH $($ TIME $)$

(75.0, 0.00), (100, 2.00), (125, 4.00), (150, 6.00), (175, 8.00), (200, 10.0), (225, 10.0)

$\mathrm{YR}=\mathrm{pYR} * \mathrm{YRn}$

$\mathrm{YRn}=\mathrm{GRAPH}(\mathrm{TIME})$

(75.0, 0.00), (100, 0.0003), (125, 0.0006), (150, 0.002), (175, 0.0058), (200, 0.01), (225, 0.00) 


\section{Chapter 10. Meaning, Use, and Limits of Simulation Models}

This material is not a textbook or a review; it cannot replace either of these. We shall simply draw the attention of the user to a few points which seem to us as particularly relevant from a practical simulation modeling point of view. Although this material is by no means trying to address philosophical issues, we shall nevertheless point at a few epistemological questions and leave them for further thoughts.

\section{Model evaluation}

Model evaluation comes first. Entire excellent books are devoted to this subject, which again, this short text cannot have the ambition to replace. Shannon (1975) posed two questions on interpretation and validation of models:

1. what is meant by establishing validity?, and

2. what criteria should be used?

In his $\mathrm{PhD}$ dissertation and a seminal article on model evaluation, Teng (1981) indicated three views that can be taken about validation:

- The rationalist view: "Rationalism holds that a model is simply a system of logical deductions made from a set of premises of unquestionable truth, which may or may not themselves be subject to empirical or objective testing. In its strictest sense, the premises are what Immanuel Kant termed synthetic a priori premises."

- The empiricist view: "The empiricist holds that if any of the postulates or assumptions used in a model cannot be independently verified by experiment, or analysis of experimental data, then the model cannot be considered valid. In its strictest sense, empiricism states that models should be developed only using proven or verifiable facts, not assumptions."

- The positivist view: "The positivist states that a model is valid only if it is capable of accurate predictions, regardless of its internal structure or underlying logic. Positivism, therefore, shifts the emphasis away from model building to model utility." 
Modeling of biological systems borrows elements from the three points of view, with varying degrees, depending on the modeling objectives. It borrows elements from the rationalist view, because we try our best to incorporate elements of reality; we are well aware, however, that the system and the corresponding model, regardless of their complexity, are only simplification of reality. It borrows elements of the empiricist view, as we try to incorporate as much experimentally measured data (as parameters or response functions) as we can; yet determining a set of genuine assumptions can be very hard, especially when one considers the stochasticity of nature; and testing the various components of a system may not necessarily yield an evaluation of an entire system, where these components interact. Lastly, it borrows elements from the positivist view, as the aim of model development is to serve a purpose; a question, however, remains as to whether any model would be acceptable, based on its sole performances, and regardless of the scientific value of its logic.

"Model evaluation" and "model validation" are often used interchangeably. The expressions however do not address the same objectives or processes (Thornley and France, 2007):

- Model evaluation is used to include all methods of critiquing a model, whereas

- Model validation is the demonstration that, within a specified domain of application, a model yields acceptable predictive accuracy over that domain. As a consequence, one has to recognize again that, being derived from an a priori view of reality, system simulation models always are incomplete, and thus model evaluation can only be incomplete as well. On the other hand, validity is not a property of a model alone, but also of the observations against which it is being tested. Thus, validation involves the use of actual data (and their inherent errors), in actual circumstances (experiments in specific set-ups, in specific situations). The perception of simulation models as "gigantic regression equations" (Thornley and France, 2007) is misguided; yet it is not surprising that efforts are still invested in developing simulation models and attempting conclusive validation. These contrasting points of views are actually linked to the objective and evaluation of models, which are briefly outlined below. 
For now, borrowing from Teng (1981), Rabbinge et al. (1983), and Thornley and France (2007), one can suggest the following steps for model evaluation:

- model verification: checking that the programming structure and the computations are performed as expected;

- visual assessment of the outputs of the model and its behavior: assessing whether the models' output conform with the expected overall behavior of a system;

- quantitative assessments of the model's outputs against numerical observations, with a large number of procedures. A starting point is to consider that common statistical testing aims at rejecting the hypothesis $\mathrm{H}_{0}$ that the distributions of observed and simulated values are identical. Testing $\mathrm{H}_{0}$ is opposite, however, to the issue of validation, where one wants to establish the sameness of outputs and observations. $\mathrm{H}_{1}$ is the hypothesis that the two distributions are different. The error of rejecting, wrongly, $\mathrm{H}_{1}$ is the main concern in model evaluation, and entails a number of approaches, for which Thornley and France (2007) provide a starting point.

\section{Evolution of models}

Modeling is an ongoing process, as new knowledge is made available, and model testing is undertaken. Penning de Vries (1982) distinguished three development phases, with:

- Preliminary models, enabling the communication, quantification, and evaluation of hypotheses. These models are developed at the frontier of knowledge, and are generally short lived. However, such models can be highly useful for the scientist in guiding research.

- Comprehensive models are built upon the former ones, as a result of knowledge accumulation. Comprehensive models are meant to be explanatory, because their structure, their parameters, and their driving functions are derived from actual experimental work and observations. Such models, however, are often large and intricate, making their communication difficult.

- Summary models are a necessary outcome of comprehensive models; they synthesize what appears to be their most important components. As stated by 
Penning de Vries (1982), "the" summary model of a comprehensive model does not exist: this depends on the depth and objective aimed at.

Table 10.1 summarizes the properties of these three types of models. One may note that the evaluation process and its ambitions will depend on the type of model.

Table 10.1. Relative values of models in different phases of development (From Penning de Vries, 1982)

\begin{tabular}{|l|l|l|l|l|}
\hline $\begin{array}{l}\text { Types of } \\
\text { models }\end{array}$ & $\begin{array}{l}\text { Predictive } \\
\text { value }\end{array}$ & $\begin{array}{l}\text { Scientific } \\
\text { value }\end{array}$ & $\begin{array}{l}\text { Instructive } \\
\text { value }\end{array}$ & Simplicity \\
\hline Preliminary & very low & very high & high & high \\
\hline Comprehensive & $\begin{array}{l}\text { moderate - } \\
\text { high }\end{array}$ & very high & low & very low \\
\hline Summary & high & low & high & very high \\
\hline
\end{tabular}

\section{Use of simulation models}

A key value of simulation models is their heuristic value: they enable the mapping of processes that are assumed to take place in a given system. Success here depends on a proper choice of system's limits and state variables, which also implies deciding which variables will be external to, i.e., independent from, the system, and be driving variables. There is a large flexibility in laying out links (as flows, or as numerical connections), or erasing them. One may see this as the sketching phase of a painter preparing her/his work. This stage, of course, is essential. This is where independence of thought and creativity lies. From it, too, will depend all the 'true' modeling work.

Laying out hypotheses is in part related to the previous stage. It involves more, however, because such hypotheses will be derived from the modeler's experience, or experiments. "No one can be a good observer unless he is a good theorizer" (Charles Darwin, quoted from Zadoks, 1972). We believe the reverse to be true too, and this is why modeling needs to combine both conceptual and actual experimental work. In doing so, the development of a (preliminary) model helps in guiding research: questions are asked, knowledge gaps are identified, and thus experiments for a purpose are designed. Modeling goes hand in hand with experimental work.

When enough hypotheses have been assembled, a next stage may consist in conducting verifications and preliminary scenario analyses: is the model behaving as one 
might expect, or does it go astray? In the latter case, one needs to re-consider the previous steps. An important effort at this stage is often to be considered: epidemiological experts often tend to look at a large number of details, and simplification is needed. This may entail the need to ponder the time constant of the processes involved and ensure that two levels of integration (in epidemiology: an epidemic and its underlying processes; in crop loss modeling: yield build-up and its determining, limiting, and reducing factors) are considered.

When one is satisfied, overall, with the model behavior, an evaluation phase is needed, as discussed above. This enables one of the most interesting outcomes of modeling, i.e., scenario analysis. Simulation modeling enables projections in possible futures. These futures may be materialized by the driving functions (quite a few plant pathologists are involved in climate change research, for instance; Garrett, 2010) or by the parameter values.

Another use of models is to conduct simulated experiments. Luo and Zeng (1995), for instance, provide a fine example of such work on components of partial resistance to yellow rust of wheat, with strong links with experimental work.

Model simplification then can lead to important outcomes from the practical standpoint. One, of course, is disease management, for which quite a number of models have been developed. One of many examples is SIMCAST (Fry et al., 1983; Grünwald et al., 2000) for potato late blight. Again, simulation modeling can become a very powerful scoping approach. Combining this potato late blight management model with a GIS, Hijmans et al. (2000) paved the way towards global geophytopathology and risk assessment. This major outcome of decades of research is one example, which should encourage plant pathologists and biologists, in general, to engage into a field which, we believe, is still full of promises.

\section{References}

Fry, W. E., Apple, A. E., and Bruhn, J. A. 1983. Ecaluation of potato late blight forecasts modified to incorporate host resistance and fungicide weathering. Phytopathology 73:1054-1059.

Garrett, K. A., Forbes, G. A., Savary, S., Skelsey, P., Sparks, A. H., Valdivia, C., van Bruggen, A. H. C., Willocquet, L., Djurle, A., Duveiller, E., Eckersten, H., Pande, S., 
Vera Cruz, C., and Yuen, J. 2010. Complexity in climate change impacts: a framework for analysis of effects mediated by plant disease. Plant Pathol. 60:15-30.

Grünwald, N. J., Rubio-Covarrubias, O. A., and Fry, W. E. 2000. Potato late-blight management in the Toluca Valley: forecasts and resistant cultivars. Plant Dis. 84:410416.

Hijmans, R. J., Forbes, G., and Walker, T. S. 2000. Estimating the global severity of potato late blight with GIS-linked disease forecast models. Plant Pathol. 49:697-705.

Luo, Y., and Zeng., S. M. 1995. Simulation studies on epidemics of wheat stripe rust (Puccinia striiformis) on slow rusting cultivars and analysis of effects of resistance components. Plant Pathol. 44:340-349

Penning De Vries, F. W. T. 1982. Phases of development of models. Pages 21-25 in: Simulation of Plant Growth and Crop Production. F.W.T. Penning de Vries and H. H. Van Laar, , eds. Pudoc, Wageningen.

Shannon, R. E. 1975. Systems Simulation: The Art and Science. Prentice Hall, NJ.

Teng, P. S. 1981. Validation of computer models of plant disease epidemics: a review of philosophy and methodology. Z. Pflanzenkrank. Pflanzensch. 88:49-63.

Thornley, J. H. M., and France, J. 2007. Mathematical Models in Agriculture. Quantitative Methods for the Plant, Animal and Ecological Sciences, 2nd ed. CABI, Wallingford.

Zadoks, J. C. 1972. Methodology in epidemiological research. Annu. Rev. Phytopathol. 10:253-276. 


\section{Instructions to Run the Simulation Models}

A series of simulation models is provided with these modules and is available on this website.

These models have been built with the STELLA® software program, and can be run using different programs, available at the ISEE systems web site http://www.iseesystems.com/

1. The models can be run and explored using the isee Player program, which can be downloaded for free. The direct link to download isee Player is:

http://www.iseesystems.com/softwares/player/iseeplayer.aspx

2. These models can also be run using the full STELLA® v10 software program, which can be purchased online at http://www.iseesystems.com/.

3. A free trial version of the STELLA program v10 can also be downloaded from the same website. The direct link to download the free trial version is: http://www.iseesystems.com/community/downloads/STELLA/STELLADemo.aspx

Two sets of models are available in this course: models that can be run with STELLA v10 (file extension .STMX), and models which can be run with STELLA v9 (file extension .STM). The latter version will enable users who use version 9 to run the models.

More information about these programs can be found at:

http://www.iseesystems.com/softwares/Education/StellaSoftware.aspx

and

http://www.iseesystems.com/community/downloads/tutorials/ModelBuilding.aspx

\section{Model Files:}

WSTELLA v9

STELLA v10.0.4 